\title{
Investigation of Material Growth and Fabrication Processes for THz Frequency SIS Mixers
}

\author{
A Dissertation \\ Presented to \\ the faculty of the School of Engineering and Applied Science \\ University of Virginia
}

In Partial Fulfillment

of the Requirements for the Degree of Doctor of Philosophy in Electrical Engineering

by
Michael Cyberey
May 2014

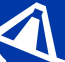

UVML NRA NRAO : .

University of Virginia

Microfabrication Laboratory

Submillimeter Research Group 


\section{APPROVAL SHEET}

The dissertation

is submitted in partial fulfillment of the requirements

for the degree of

Doctor of Philosophy

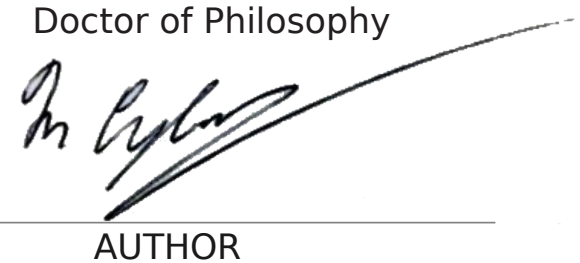

The dissertation has been read and approved by the examining committee:

Arthur W. Lichtenberger

Advisor

Robert M. Weikle II

Jiwei Lu

Anthony R. Kerr

Nathan Swami

\section{Aaron Datesman}

Accepted for the School of Engineering and Applied Science:

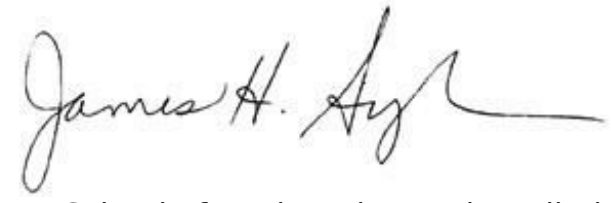

Dean, School of Engineering and Applied Science

May

2014 


\section{Dedication}

It is with my utmost gratitude that I dedicate this dissertation to all those who contributed to my education, both inside and outside of the classroom and lab. You've shared with me the concrete and abstract concepts that have shaped my understanding of the physical world and developed my appreciation of those things yet to be explained by science. You've instilled in me a genuine love of research and the pursuit of knowledge from which I have committed my academic endeavors and hope to continue beyond the walls of this illustrious institution. 


\section{Contents}

Abstract $\quad$ v

Acknowledgements vii

Dedication viii

1 Introduction 1

$1.1 \mathrm{THz}$ Technology and Superconducting Devices . . . . . . . . . 1

1.2 Outline of Dissertation . . . . . . . . . . . . . . . 4

2 Overview of SIS and Fabrication $\quad 6$

2.1 Superconductivity and SIS tunnel junctions $\ldots \ldots \ldots$

$2.2 \mathrm{THz}$ Detection . . . . . . . . . . . . . . . . . . . . . 12

2.3 Requirements for Higher Frequency Operation . . . . . . . . . . 16

2.3 .1 Substrate Requirements . . . . . . . . . . . . . . 16

2.3.2 Superconducting Material Requirements . . . . . . . . . 19

2.3.3 Barrier Requirements and Junction Geometry . . . . . . . . 21

3 Trilayer Deposition and Barrier Formation 24

3.1 Background of SIS Trilayer Growth . . . . . . . . . . . . 24

3.2 Modifications to Deposition System . . . . . . . . . . . 26

3.2.1 Reconfiguration of the ICP source and Addition of an Ion Gun 27 
3.2.2 Isolation of Load Lock and Addition of RGA and Ellipsometer 30

3.3 SIS Trilayer Growth and RnA Results . . . . . . . . . . . . . . . . 31

3.4 ALMA Band-8 Results . . . . . . . . . . . . . . . . . . . . 33

3.4.1 Post Fabrication Device Damage . . . . . . . . . . . . . . 34

3.4 .2 Noise Temperature and IV results . . . . . . . . . . . . . . . . 35

3.5 Conclusions . . . . . . . . . . . . . . . . . . 36

4 Al Quadlevel Junction Fabrication and SIS Test Mask 38

4.1 Introduction . . . . . . . . . . . . . . . . . . . . . 38

4.1.1 Background of Modern SIS Junction Fabrication . . . . . . . . 40

$4.2 \mathrm{Al}$ Quadlevel Junction Fabrication . . . . . . . . . . . . . . . . . . 46

4.2.1 Polymers and Al Cap . . . . . . . . . . . . . . . . . . . . 47

4.2 .2 Aluminum Etching . . . . . . . . . . . . . . . . . . 48

$4.2 .3 \quad$ NFR/Polyimide Etching . . . . . . . . . . . . . . . . 50

4.2.4 Nb/Au Top Electrode Etch and Junction Insulation . . . . . . 52

4.3 New SIS Test Mask . . . . . . . . . . . . . . . . . . . 55

4.3.1 Wiring Layer and Junction Insulation Test Block . . . . . . . 59

4.3.1.1 Wiring Layer Fingers . . . . . . . . . . . . . . 60

4.3.1.2 $\quad \mathrm{SiO}_{2}$ Edge and side Overlap Coverage . . . . . . . 61

4.3.1.3 $\mathrm{SiO}_{2}$ Pinholes ................ 61

4.3.2 Transition Temperature $\left(T_{c}\right)$ Block . . . . . . . . . . . 62

4.3.3 Dielectric Measurement Block . . . . . . . . . . . . . . 64

4.4 Results from Al Quadlevel Process and New Test Mask . . . . . . . . 64

4.4 Testing Setup . . . . . . . . . . . . . . . 66

4.4.2 Comparison of Different $\mathrm{SiO}_{2}$ Deposition Methods . . . . . . . 67

4.4.2.1 Ellipsometry Analysis . . . . . . . . . . . 70

4.4.2.2 Junction Liftoff Profile . . . . . . . . . . . . . 72

4.4.2.3 IV Results and Junction Quality . . . . . . . . . 75 
4.5 Conclusions and Future Work . . . . . . . . . . . . . . . . 81

5 In Situ Spectroscopic Ellipsometry of Barrier Formation 84

5.1 Introduction . . . . . . . . . . . . . . . . . . . . 84

5.2 Ellipsometric Determination of Film Properties . . . . . . . . . . 86

5.2.1 Electromagnetic Waves and Polarization . . . . . . . . . . 86

5.2.2 Reflections from Thin Films . . . . . . . . . . . . . . . 88

5.2.3 The Ellipsometer . . . . . . . . . . . . . . . . . 90

5.2.3.1 Single Wavelength vs Spectroscopic Ellipsometry . . 93

5.3 Ellipsometric Model and Parameters . . . . . . . . . . . . . . . 94

5.3.1 Modeling Dispersions of Thin Films . . . . . . . . . . . . . . . 94

5.3.1.1 Metallic Films . . . . . . . . . . . . . . . . . 94

5.3.1.2 Insulating Films . . . . . . . . . . . . . . . . . . 95

5.3.1.3 Surface Roughness . . . . . . . . . . . . . . 96

5.4 Experimental Setup and Proposed Model . . . . . . . . . . . . . . . 97

5.4.1 Modeling Al and AlN Films . . . . . . . . . . . . . . 99

5.4.2 Sources of error in AlN Thickness . . . . . . . . . . . . . . . . 102

5.4.3 Proposed Model for AlN Growth . . . . . . . . . . . . . . . 105

5.5 Results and Conclusions . . . . . . . . . . . . . . . . 108

5.5.1 Comparisons of Models . . . . . . . . . . . . . . 108

5.5.2 In Situ monitoring of AlN growth . . . . . . . . . . . . 111

5.5.3 Conclusions . . . . . . . . . . . . . . . . . . 113

6 Spectroscopic Analysis of ICP Nitridation 116

6.1 Introduction . . . . . . . . . . . . . . . . 116

6.2 ICP Nitridation of AlN . . . . . . . . . . . . . . . . . 117

6.3 Experimental Setup . . . . . . . . . . . . . . . . . 121

6.4 Results.......................... 124 
6.4.1 Effects of Processing Parameters on Dissociation . . . . . . . . 126

6.4.1.1 Pressure . . . . . . . . . . . . . 126

6.4.1.2 RF Power . . . . . . . . . . . . . 127

6.4.1.3 DC Current . . . . . . . . . . . . . 128

6.4.2 Effects of Dissociation on AlN Growth . . . . . . . . . . . . . 129

6.4.3 Effects of Dissociation on SIS Junction Quality . . . . . . . . 130

6.5 Summary and Conclusions . . . . . . . . . . . . . . . . . . 132

7 Conclusions and Future Work $\quad 141$

$\begin{array}{ll}\text { Bibliography } & 154\end{array}$

$\begin{array}{lll}\text { I On-wafer Cryogenic Screening } & 166\end{array}$

II Investigation of Directly Deposited AIN Barriers 176

IIIModifications to Trilayer Deposition System 183

IV Al Quadlevel Process Sheets and New Test Mask Info 195

V Source Code and Documentation 211 


\section{Abstract}

Research and development of $\mathrm{THz}$ electronics seeks to comprehend and utilize one of the last uncharted regions of the electromagnetic spectrum. Sandwiched directly between the microwave and far-infrared regions, $\mathrm{THz}$ devices often involve a hybrid fusion of optical and small-scaled RF technologies, frequently requiring novel design, materials, and fabrication techniques. Despite the increased complexity, when compared to many well-established RF and optical technologies, THz receivers used in telescope imaging applications are of great importance to radio astronomers, with current large scale multinational radio telescope projects, such as the Atacama Large Millimeter/sub-millimeter Array (ALMA) in Chile designed to observe the universe from 31 to $950 \mathrm{GHz}$ - the field of radio astronomy will be one of the immediate benefactors from the ongoing research of higher frequency $\mathrm{THz}$ detection.

Heterodyne receivers, based on the $\mathrm{Nb} / \mathrm{Al}-\mathrm{Al}_{2} \mathrm{O}_{3} / \mathrm{Nb}$ SIS tunnel junction, have demonstrated sensitivities approaching the quantum limit, making them ideal for highsensitivity $\mathrm{THz}$ detectors. However, as design frequencies approach $1 \mathrm{THz}$ and beyond, both the $\mathrm{Nb}$ electrodes and $\mathrm{Al}_{2} \mathrm{O}_{3}$ tunnel barrier of the prototypical $\mathrm{Nb} / \mathrm{Al}-$ $\mathrm{Al}_{2} \mathrm{O}_{3} / \mathrm{Nb}$ SIS junction begin to demonstrate poor performance. A turning point in SIS technology was through the development of ICP grown AlN as an alternative barrier material, first reported by our research group in 2007. However, due to run to run variation, our original ICP configuration made realization of AlN based SIS mixers impossible at the time.

In this work, we present the modifications of our trilayer deposition system that allowed our research group to realize AIN based SIS mixers meeting all ALMA Band-8 specifications. Additionally, we report on a novel fabrication process that significantly 
reduced fabrication time of high-quality SIS test devices. ICP nitridation of $\mathrm{Al}$ overlayers is inherently a complex process, and little is reported in the literature as to what affects AlN growth. With this in mind, we report on detailed investigations of ICP nitridation, using ellipsometry and spectroscopic analysis, and provide a better understanding of the processes involved in the realization of high-quality ICP grown AlN based SIS trilayer. 


\section{Acknowledgments}

First and foremost, I extend the utmost gratitude and appreciation to my advisor, Arthur Lichtenberger, whose support, guidance, and patience enabled me to pursue and accomplish the work presented in this thesis. He encouraged freedom and creativity in how I approached my work, and stressed the importance of balance in my life. His philosophy as an advisor has helped me grow as both a scientist and as an individual. I will always be grateful for the potential he saw in me and the opportunity he gave me.

I also extend my appreciation to both NRAO and the estate of Grote Reber for their financial support as I completed my PhD research.

A special thanks goes to everyone who worked behind-the-scenes at UVML. The microelectronics facilities manager, Joseph Beatrice, for going above and beyond in maintaining all aspects of laboratory operation and, first and foremost, ensuring our safety; Jian-Zhong Zhang and Jie Wang, their countless hours spent in the cleanroom perfecting the fabrication of our device circuitry did not go unnoticed; Alex Lobo, who spent incalculable hours repairing, troubleshooting, and modifying necessary lab equipment; Harry Wade, for aiding with operation and maintenance of lab equipment; Tim Pernell, for sharing his wisdom of high vacuum systems. All of these people truly fostered learning and understanding through their example and never wavered with their assistance, answering even the most rudimentary of my questions with complete attention and concern.

A very special thanks goes to Gina Marie Mattei and her family. For the past $20+$ years, they have always believed in me, and ultimately gave me the confidence to pursue my dreams. 


\title{
List of Terms
}

\author{
$\AA \quad$ - angstrom, unit of measurement equal to $1 \mathrm{e}-10$ meters \\ AC - alternating current \\ ALMA - Atacama Large Millimeter/sub-millimeter Array \\ AU $\quad-$ arbitrary units \\ $\vec{B} \quad-$ magnetic field density vector \\ BCS - Bardeen, Cooper and Schrieffer, referring to their microscopic theory of \\ superconductivity \\ BOX $\quad-$ buried oxide \\ c $\quad-$ speed of light in a vacuum \\ CMP - chemical mechanical planarization \\ CPW - coplanar waveguide \\ DC - direct current \\ DSB - double-sideband \\ $\vec{E} \quad-$ electric field density vector \\ e $\quad-$ charge of an electron \\ $\epsilon_{0} \quad-$ electric constant \\ $\epsilon_{F} \quad-$ Fermi energy level \\ $\epsilon_{i}, \epsilon_{j} \quad-$ energy of states with momenta $\mathrm{p}_{i}$ and $\mathrm{p}_{j}$




\begin{tabular}{|c|c|}
\hline HEB & - hot electron bolometer \\
\hline ICP & - inductively coupled plasma \\
\hline$\lambda_{L}$ & - London penetration depth \\
\hline $\mathrm{J}_{C}$ & - critical current density \\
\hline MSE & - mean squared error \\
\hline$\rho$ & - resistivity \\
\hline $\mathrm{R}_{N}$ & - normal state resistance of an SIS tunnel junction \\
\hline $\mathrm{R}_{N} \mathrm{~A}$ & - normal state resistance and junction area product \\
\hline $\mathrm{R}_{S G}$ & - subgap resistance of an SIS tunnel junction \\
\hline $\mathrm{RD}$ & - relative dissociation \\
\hline $\mathrm{RF}$ & - radio frequency \\
\hline RIE & - reactive ion etching \\
\hline SDMRG & - Superconducting Devices and Materials Research Group \\
\hline SIS & - superconductor insulator superconductor \\
\hline SSB & - single-sideband \\
\hline TIM & - thermal interface material \\
\hline TRL & - through line reflect \\
\hline$\mu_{0}$ & - magnetic constant \\
\hline UVML & - University of Virginia Microfabrication Laboratories \\
\hline VNA & - Vector Network Analyzer \\
\hline
\end{tabular}




\section{Chapter 1}

\section{Introduction}

\subsection{THz Technology and Superconducting Devices}

Research and development of THz electronics seeks to comprehend and utilize one of the last uncharted regions of the electromagnetic spectrum, from roughly $\sim 100 \mathrm{GHz}$ to $\sim 3 \mathrm{THz}$. The THz spectrum is sandwiched directly between the microwave and far-infrared regions. As a result, $\mathrm{THz}$ devices are often a hybrid fusion of optical and small-scaled RF technologies, frequently requiring novel design, materials, and fabrication techniques.

Despite the increased complexity of device design and fabrication, when compared to many well-established RF and optical technologies, $\mathrm{THz}$ radiation has unique properties spawning interest in current research, notably imaging applications. When compared to optical imaging, $\mathrm{THz}$ radiation has the ability to penetrate many materials and makes such devices suitable candidates for security screeners at airports to detect concealed contraband such as weapons or explosives. When compared to standard x-ray imaging, $\mathrm{THz}$ radiation is non-ionizing and, as a result, is finding broader application in medical research imaging, notably as diseased tissues have recently been shown to have unique $\mathrm{THz}$ spectral signatures when compared to their healthy 
counterparts [1]. In addition to these applications, $\mathrm{THz}$ receivers used in telescope imaging applications are of great interest to radio astronomers. As much as $98 \%$ of the photons emitted since the big bang are in the $\mathrm{THz}$ region and the imaging of interstellar matter allows astronomers to chart-out the interstellar composition of the universe [2].

Receivers designed to detect radiation in the $\mathrm{THz}$ spectrum fall into two general categories, direct and coherent (heterodyne) detection. Direct detectors measure the intensity of incident radiation through the absorption of photons, generating an electrical response through localized heating of a temperature dependent material or generation of DC currents in a detector; as such, the intensity of the signal is detected, but all phase information of the radiation is lost. Coherent detectors function by mixing (heterodyning) a signal with a local oscillator, and shifting frequency content down to lower $(\mathrm{GHz})$ frequencies where low noise amplifiers and analog to digital converters (ADC) are readily available. A benefit of coherent detection is that all phase information of the source is preserved, which allows the use of phase-dependent techniques such as interferometry using multiple receivers. Both direct and heterodyne detection are discussed in greater detail in the following chapter. However, in this work we focus on heterodyne receiver applications and the mixing element that must be able to efficiently operate at $\mathrm{THz}$ frequencies.

Non-superconducting THz mixer technologies exist, such as Schottky diode based mixers. Schottky diodes possess the current-voltage nonlinearity required for heterodyne mixing at room and cryogenic temperatures and have been implemented in many $\mathrm{THz}$ applications from receivers to compact $\mathrm{THz}$ sources. Although Schottky diode mixers are ideal for certain $\mathrm{THz}$ applications such as room-temperature vector network analyzers, limiting factors for ultra low noise $\mathrm{THz}$ receiver applications are the relatively high noise temperature and LO power requirements when compared to superconducting based mixers at certain frequencies. With this in mind, 
lower-power superconducting circuits have found important applications in THz heterodyne receivers where sensitivity is paramount. There are two main competing superconducting mixer technologies, namely the Hot Electron Bolometer (HEB) and Superconducting-Insulating-Superconducting (SIS) junction. HEBs can successfully operate up to around $3 \mathrm{THz}$ as a heterodyne mixers with low $\mathrm{LO}$ power requirements that can be met by compact, commercially available, tunable THz sources. However, there are limiting factors such the bandwidth, limited to a few $\mathrm{GHz}$, and higher noise temperatures compared to SIS mixers (although current SIS mixer technologies are generally limited to $\sim 1 \mathrm{THz}$ ). SIS based heterodyne receivers have been shown to possess sensitivities approaching the quantum limit and as a result, the work horse of most high-sensitivity $\mathrm{THz}$ receivers is the SIS tunnel junction.

However, as we press the limits of conventional SIS technology to realize higher frequency mixers, the need for new processing and material growth techniques becomes apparent. A main goal of this work is the further development of SIS technology allowing the design and fabrication of SIS based heterodyne mixers operating at frequencies approaching $1 \mathrm{THz}$, utilizing novel material growth and fabrication techniques. In addition to $\mathrm{THz}$ frequency electronics and imaging applications, the research, characterization, and development of high-quality superconducting thin films and SIS junctions covered in this work have wide applications to the broad field of superconductivity. Notably, current research in quantum computing and non-volitile superconducting memory rely on the ability to produce high quality superconducting films and Josephson junctions $[3,4,5,6]$. As such, we note that the deeper understanding of the various aspects involved in the realization and characterization of SIS devices gained in this work can be applied to various applications outside the direct scope of $\mathrm{THz}$ frequency heterodyne mixers. 


\subsection{Outline of Dissertation}

The next chapter introduces the basics of superconductivity required to understand the current-voltage characteristics of SIS junctions and the basic theory required to understand their application as THz frequency heterodyne mixing elements. Previous work, covered in greater detail in my previous thesis, is discussed and building upon this work, current limitations of and potential improvements to our processes, to be investigated as part of this work, are introduced.

Chapter 3 discusses our trilayer deposition system and our chosen materials for both superconducting electrodes, insulating barriers, and their methods of growth. Issues that arose when I first began this work are presented and the modifications to the trilayer deposition system, to alleviate these issues, are discussed in detail. After the modifications were implemented, improvements to the quality and repeatability of our SIS trilayer are presented, as well as the results of ALMA Band- 8 mixers, fabricated on $3 \mu \mathrm{m}$ thick SOI substrates at UVML

The material presented in Chapter 3 explains the attractiveness of both reducing the fabrication time for realization of SIS test devices and developing a processes where individual aspects of SIS junction fabrication can be investigated. Noting this, Chapter 4 discusses the development of a novel mask set and junction fabrication process to both reduce fabrication time, and isolate individual components of the process flow of SIS junctions for investigation.

Chapter 5 begins with a brief review of electromagnetic waves, designed to give the reader an understanding of the basic principles of ellipsometry. Building upon this, we present our investigations on the use of spectroscopic ellipsometry to in situ monitor AlN barrier growth by plasma nitridation of $\mathrm{Al}$ overlayers, through use of an ellipsometric model developed as part of this work.

In Chapter 6, we investigate the use of an optical spectrometer, coupled to our trilayer deposition tool, to determine the relative dissociation of the plasma used for 
growth of our AlN barrier layers. The development of the algorithm used to determine dissociation is presented, and the correlations between plasma disassociation and both AlN growth rates and the quality of the SIS trilayer grown by varying degrees of dissociation are discussed.

The final chapter summaries the contributions and general conclusions made as a result of this work. We also present ideas and discuss potential areas of investigation that stem from the work presented in the dissertation. The final appendices cover initial studies performed during my time at UVML, including the use of directly sputtered AlN tunnel barriers for use with SIS trilayer with NbTiN base electrodes, and on wafer cryogenic screening of SIS mixer wafers. Also included is all the code used throughout this work, the CAD for system modifications performed in Chapter 3, and the processing sheets used for the Al Quadlevel process developed in Chapter 4. 


\section{Chapter 2}

\section{Overview of SIS and Fabrication}

\subsection{Superconductivity and SIS tunnel junctions}

SIS tunnel junctions consist of two superconducting electrodes separated by a thin, on the order of a nanometer thick, insulating barrier. They ideally possess a sharp current-voltage non-linearity upon onset of quasi-particle tunneling at a sum-gap voltage and are often employed as heterodyne mixing elements. Their operation is briefly discussed below, for a more detailed explanation the author recommends an elegant yet thorough approach provided by Van Duzer [7].

In 1911, through the use of the recently discovered ability to liquefy helium, Heike Kamerlingh Onnes began investigating the properties of materials at temperatures approaching absolute zero. By measuring the resistivity through a U-shaped vial filled with mercury, Onnes noticed an odd phenomenon. The resistivity of the mercury slowly decreased with decreasing temperature, as expected for a metal, but when the temperature reached $4.2 \mathrm{~K}$, the resistivity sharply dropped to an unmeasurable amount. Initially thought to be the result of faulty electrical connections, the experiment was repeated, producing similar results. This observed sharp transition to zero resistance represented the discovery of the phenomenon named superconductivity. 


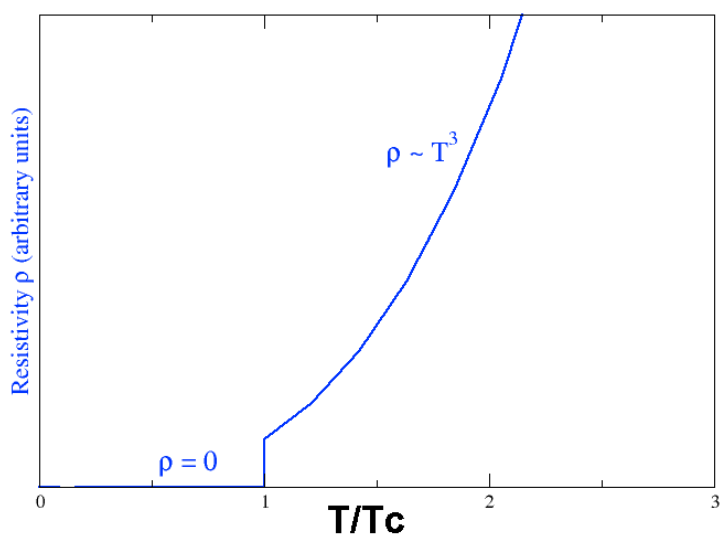

Figure 2.1: Characteristic plot of resistivity vs temperature for a superconducting sample. Resistance drops with temperature through reduced scattering events, but when a critical temperature is reached, resistance abruptly drops to zero.

A superconducting material is characterized by a transition to a zero resistivity state when cooled below a transition temperature, $T_{c}$, as shown in Figure 2.1. It has been shown that two electrons can interact with each other through phononexchange (scattering) and this interaction between electrons can be attractive [8]. Theory suggests this attraction is maximized (energy is minimized) when the coupled electrons have equal and opposite momenta - below $T_{C}$, this interaction is favorable and coupled electrons form what is referred to as a Cooper pair. Cooper pairs contain 2 electrons, hence the total spin is no longer half-integer, and the bound particles behave not as Fermions but rather Bosons. The overall implication is that Cooper pairs, unlike single electrons, follow Bose-Einstein statistics and can occupy a single energy level. We also note that there is an energy gap associated with the formation of Copper pairs which equates to the minimum energy required to break the pair. When a Cooper pair consisting of electrons with equal and opposite momenta in states $\mathrm{p}_{i} \uparrow$ and $-\mathrm{p}_{i} \downarrow$ is broken (arrow indicating electron spin), the two electrons occupy the states $\mathrm{p}_{i} \uparrow$ and $\mathrm{p}_{j} \uparrow$ where the complimentary states, $\mathrm{p}_{i} \downarrow$ and $-\mathrm{p}_{j} \downarrow$, required to form Cooper pairs, are empty. BCS theory predicts the required energy for this process is [9]:

$$
E=\left(\left(\epsilon_{i}-\epsilon_{F}\right)^{2}+\Delta^{2}\right)^{\frac{1}{2}}+\left(\left(\epsilon_{j}+\epsilon_{F}\right)^{2}+\Delta^{2}\right)^{\frac{1}{2}}
$$


Where $\epsilon_{i}$ and $\epsilon_{j}$ are the energy states associated with momenta $\mathrm{p}_{i}$ and $\mathrm{p}_{j}, \epsilon_{F}$ is the Fermi energy level and $\Delta$ is the binding energy of an electron in the Cooper pair. The minimum energy required to break a Copper pair is referred to as the material's characteristic energy gap, $E_{g a p}$, and from (2.1) $E_{g a p}=2 \Delta$. From this, one can construct a simplified energy-band diagram of an SIS junction. For the superconducting regions, we can take a quasi-particle semiconductor analogy.

When a material enters the superconducting state, a band gap is opened characterized by the energy gap of the superconductor. Therefore, there are two energy bands separated by $E_{g a p}$; at $0 \mathrm{~K}$, the lower band contains superconducting electrons and the upper band contains empty electron states. The superconducting density of states, according to BCS theory, follows the functional dependence (setting the $E=0$ reference at the Fermi level) [10]:

$$
N_{s}(E)= \begin{cases}\frac{N_{n} E}{\left(E^{2}-\Delta^{2}\right)^{1 / 2}} & |E| \geq \Delta \\ 0 & |E|<\Delta\end{cases}
$$

where $N_{n}$ is the normal density of states following the familiar $E^{1 / 2}$ dependence and can be taken as a constant in our range of interest for SIS junctions with band gaps on the order of meV. If we separate two superconductors by a thin insulating barrier, as shown in Figure 2.2, at $\mathrm{T}=0 \mathrm{~K}$, ideally current can only flow through the barrier via quantum mechanical tunneling. If we assume two superconductors separated by a thin barrier of height, $U$, and thickness, $L$, we can solve for the approximate tunneling probability:

$$
T \propto e^{-2 k L}
$$

where

$$
k=\frac{\sqrt{2 m(U-E)}}{\hbar}
$$


An expression for quasi-particle tunneling current can be derived taking into account the tunneling probability, a factor, $A_{g}$, which is a constant taking into account the physical geometries, and the Fermi-Dirac distribution $f(E)$ as:

$$
\left.I=T A_{g} \int N_{s 1}(E) N_{s 2}\left(E-e V_{\text {bias }}\right)\left(f\left(E-e V_{\text {bias }}\right)-f(E)\right)\right) d E
$$

where $N_{s 1}$ and $N_{S 2}$ are the density of states for the respective superconductors defined in (2.2). To interpret this equation, one notes that in order for an observable tunneling current to flow, two conditions must be met: (1) the barrier must be thin enough to have appreciable probability of tunneling (factor $T$ ), and (2) there must be empty states on the opposite side of the barrier into which the carriers may tunnel $\left(N_{s}\right.$ defines states, $f(E)$ defines occupancy). If both metals are non-superconducting, $N_{s}$ reduces to $N_{n}$ and we observe an approximately linear resistance proportional to the tunneling probability as shown by the dashed line in Figure 2.2. However, let us assume both metals are in the superconducting state, condition (1) is met, and there is a small bias applied to our junction which has a magnitude less than the sum-gap voltage, $V_{\text {gap }}=2 \Delta / q$, where $q$ is the charge of an electron. With an applied bias less than the sum-gap voltage, the superconducting band on the left is lined up in the middle of the energy gap on the right and there are no empty states for the carriers to tunnel from left to right and current will not flow. It is not until the applied bias reaches the gap voltage, uncovering empty states, that current will flow. The density of states shown in (2.2) contains a singularity around the band edges. At $0 \mathrm{~K},(2.3)$ produces zero current below sum-gap bias voltage, the characteristic highly nonlinear current rise at sum-gap bias voltage, followed by an approximately linear dependence as shown in Figure 2.2.

While Figure 2.2 shows an idealized SIS IV characteristic at $0 \mathrm{~K}$ based on the quasi-particle model described above, this is not the complete picture of an SIS IV. In addition to single electrons, tunneling of Coopers pairs across the barrier can occur. 


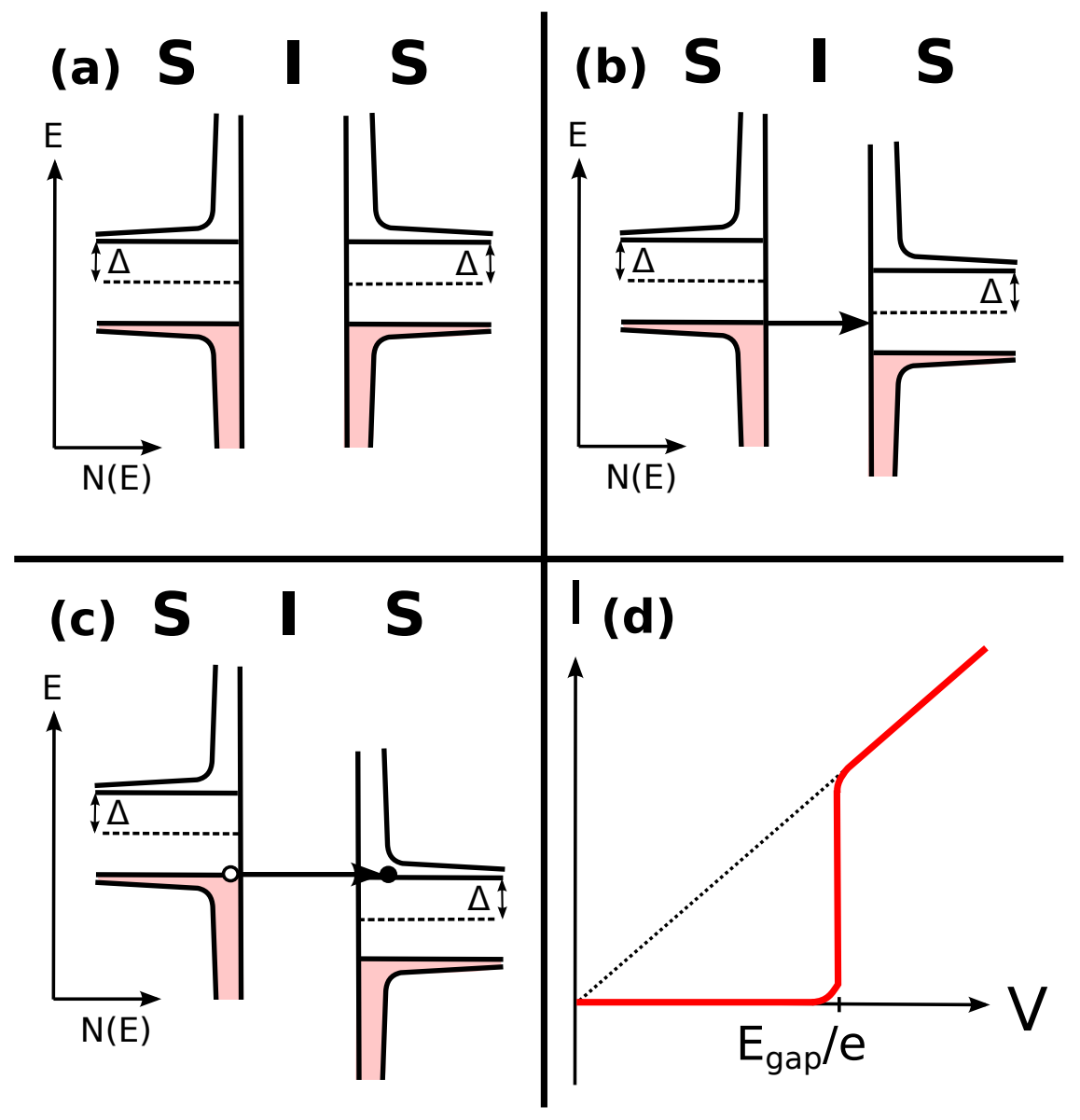

Figure 2.2: Simplified IV characteristics of SIS tunnel junction. (a) Under zero-bias, one sees the equilibrium energy band diagram. Grey areas represent the occupied density of states. (b) At bias less than the gap voltage, there are no states for the quasi-particles to tunnel into and hence no current flow. (c) When the bias is increased to the gap voltage, the quasi-particles can tunnel across the thin barrier and cause a sharp current rise. (d) The resulting IV curve, demonstrating a sharp non-linearity at gap voltage. The dashed line indicates the IV characteristic if the two electrodes where in the normal state. 
This phenomenon is referred to as the Josephson Effect, predicted in 1962 by Brian David Josephson for which he later received the Nobel prize, and is described by the equations [11]:

$$
\begin{gathered}
I_{s}=I_{c} \sin (\phi) \\
\nu=\frac{2 e}{h} V_{d c}
\end{gathered}
$$

Equation 2.4 describes the DC Josephson Effect, where a super current $\left(I_{s}\right)$ proportional to the phase difference $(\phi)$ of the two superconducting wave functions on either side of the barrier, can flow with zero voltage differential across the barrier until a maximum critical current $\left(I_{c}\right)$ is exceeded. Equation 2.5 describes the AC Josephson Effect, where a frequency of oscillation $(\nu)$ is observed as emission of radiation or a ripple in the IV when a junction is biased $\left(V_{d c}\right)$. The DC Josephson effect is readily observed as a zero-voltage current with resulting hysteresis while DC testing of SIS junctions. The IV from a Nb/Al- $\mathrm{Al}_{2} \mathrm{O}_{3} / \mathrm{Nb}$ junction recorded at $4.2 \mathrm{~K}$, by submersion in liquid helium, is shown in Figure 2.3. As the junction is current-biased, started at $V_{j}=0$, the current increases until $I_{c}$ is exceeded, then the voltage jumps to the corresponding value of the quasi-particle current rise (captured by the $10 \mathrm{~ms}$ sampling rate of our data logger). If we sweep, starting at current value in excess of $I_{c}$, the quasi-particle IV is captured. This results in the hysteretic loop indicated by the arrows shown in Figure 2.3. We note that the Josephson current is often suppressed through use of a applied magnetic field for SIS mixer applications, however, for the IVs recorded by dip testing individual junctions in this work, no active suppression is performed and the DC Josephson current is often observed. 


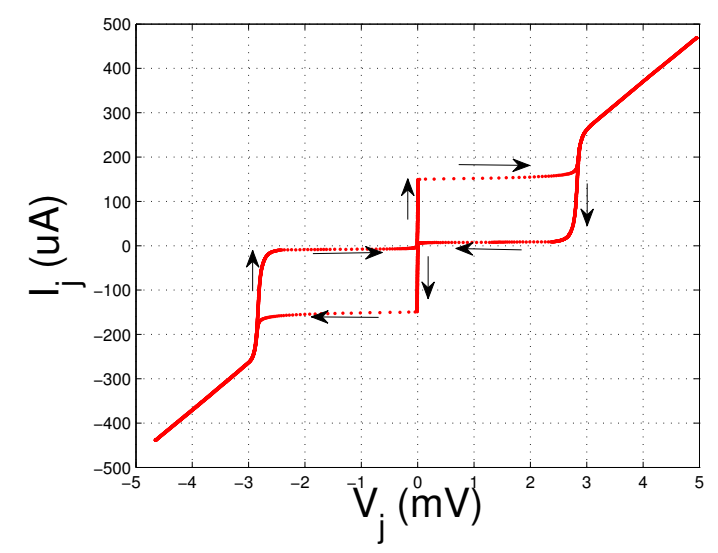

Figure 2.3: $\mathrm{Nb} / \mathrm{Al}-\mathrm{Al}_{2} \mathrm{O}_{3} / \mathrm{Nb}$ junction fabricated and tested, at $4.2 \mathrm{~K}$, as part of this work. We note the presence of the DC Josephson super current at 0 VDC bias, and the resulting hysteresis.

\section{$2.2 \mathrm{THz}$ Detection}

As mentioned in Chapter 1, there is a growing interest in the field of $\mathrm{THz}$ electronics for imaging applications. Noting current large scale multinational radio telescope projects, such as the Atacama Large Millimeter/sub-millimeter Array (ALMA) in Chile, ALMA currently captures images of the sky using all available $\mathrm{THz}$ atmospheric windows up to $1 \mathrm{THz}$ and there are proposals to extend operation up to 1.5 $\mathrm{THz}$; the field of radio astronomy will be one of the immediate benefactors from the ongoing research of higher frequency $\mathrm{THz}$ detection [12]. Detectors are generally split into two categories: direct (power) detection and coherent (heterodyne) detection. Power detectors of $\mathrm{THz}$ radiation rely on localized heating from impinging photons changing the impedance of a receiver circuit. Such examples of devices that can operate as direct detectors are HEBs (localized heating), kinetic inductance detectors (photon breaking of Cooper pairs causing a frequency shift of a resonant structure), or Schottky barrier diodes detectors (generation of currents). However, direct detection is non-coherent - phase information of the source is lost, and unsuitable for interferometer arrays. Coherent detection is therefore an integral tool in the field of radio astronomy when high angular resolution and point-source sensitivity are required. For 
this reason, this dissertation will focus on SIS based $\mathrm{THz}$ frequency mixers designed for use in heterodyne receivers, as their performance is unparalleled for frequencies up to $\sim 1 \mathrm{THz}[2]$.

High quality analog-to-digital converters (A/D), filters, and low noise amplifiers are readily available and well characterized at GHz frequencies, however, similar technologies do not exist for $\mathrm{THz}$ bands. Heterodyning is the process of frequency shifting a high frequency signal of interest to a lower intermediate frequency (IF) through mixing with a local oscillator (LO), while maintaining all phase information. To understand the most general operation of a classical heterodyne receiver utilizing a non-linear resistive element, one notes a function can be expressed in the Taylor Series:

$$
\sum_{n=0}^{\infty} \frac{f^{(n)}(a)}{n !}(x-a)^{n}
$$

For any non-linear function, there will be terms where the input of the function is multiplied by itself. For simplicity, let us take the simplest case of the function, $f=x^{2}$, which takes the form of the 2 nd term of the Taylor series. If an input to this function is the time varying signal $s_{i n}(t)+s_{L O}(t)$, the result becomes:

$$
f\left(s_{i n}(t)+s_{L O}(t)\right)=\left(s_{i n}(t)+s_{L O}(t)\right)^{2}=s_{i n}^{2}(t)+2 s_{i n}(t) s_{L O}(t)+s_{L O}^{2}(t)
$$

Focusing on the second term, we note that multiplication in time domain corresponds to convolution in the frequency domain (denoted by upper case letters):

$$
s_{i n}(t) s_{L O}(t)=S_{i n}(\nu) * S_{L O}(\nu)
$$

Let use assume $s_{L O}$ is the sinusoidal LO, $\cos (\omega t)$, and $s_{i n}$ is our signal of interest for detection; (2.8) becomes: 

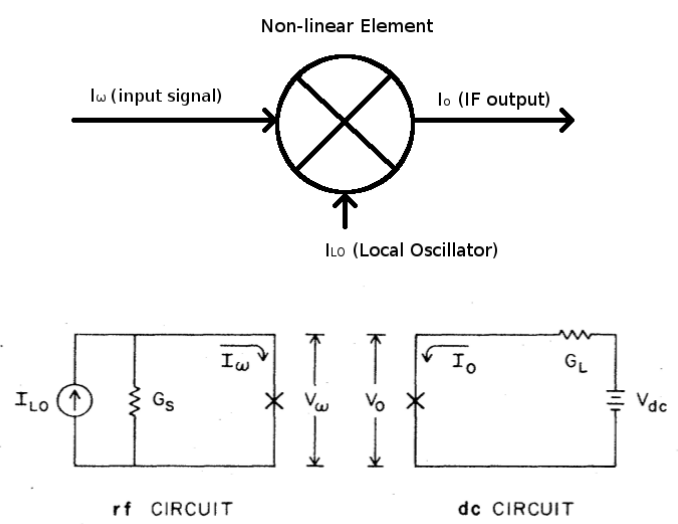

Figure 2.4: Top: Standard block diagram of a mixing element, Bottom: Circuit diagram of simplified equivalent circuit of a mixer, assuming the phase and frequency of the input signal and LO are matched IE amplitude modulation with DC output (Tucker and Feldman, 1985). The RF (high frequency) and DC (IF) circuits are separated. The LO is represented by the Norton circuit, $I_{L O}$ and $G_{s}$, the DC bias on the mixer by the Thévenin circuit, $V_{d c}$ and $G_{L}$, and $I_{\omega}, V_{\omega}, I_{o}, V_{o}$ represent the currents and voltages of the mixing element by both the RF and DC.

$$
S_{i n}(f) * \frac{1}{2}\left[\delta\left(\nu+\nu_{L O}\right)+\delta\left(\nu-\nu_{L O}\right)\right]=\frac{1}{2}\left[S_{i n}\left(\nu+\nu_{L O}\right)+S_{i n}\left(\nu-\nu_{L O}\right)\right]
$$

From (2.9), one can see that the necessary requirements for heterodyne mixing are a non-linear device and a LO with a frequency close to that of the source. An equivalent circuit, proposed by Tucker and Feldman, of a simplified mixer is shown in Figure 2.4 [13]. A key result of analysis of this circuit is that conversion gain, defined as the ratio of IF power to the RF signal power, is strongly dependent on the non-linearity of the mixing element, and limited to unity for single-sideband (SSB) mixers. A more in depth analysis is provided by Torrey and Whitmer, but offers the same result as Tucker and Feldman $[13,14]$.

However, a classical analysis is only appropriate for SIS junctions when the photon energy $(h \nu / e)$ is much smaller than the voltage scale of the nonlinearity. When the SIS IV curve shown in Figure 2.2 is biased within $n h \nu / e$ of the sum-gap voltage, $n$ 

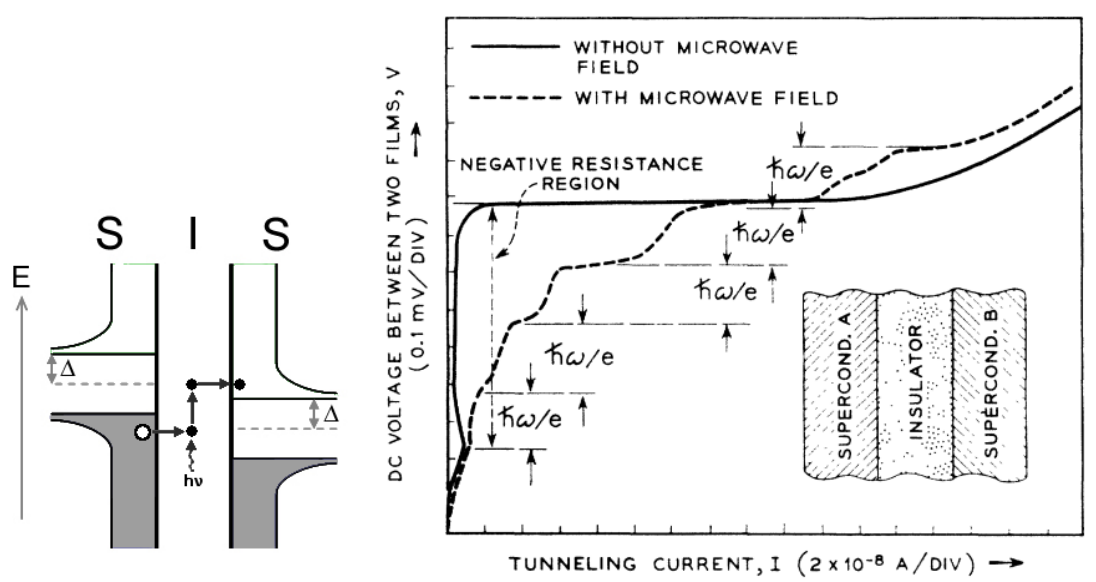

Figure 2.5: Left: Photon assisted tunneling as shown by quasi-particle energy band diagram. Right: Experimental IV characteristics of an $\mathrm{Al} / \mathrm{Al}_{2} \mathrm{O}_{3} /$ In SIS junction illuminated by $38 \mathrm{GHz}$ radiation, showing photon assisted tunneling steps. (Tien and Gordon, 1963)

photon(s) will have enough energy to excite a quasi-particle across the gap, producing a photon-assisted tunneling current, as shown in Figure 2.5 [15]. A quantum generalization of classical mixer theory, proposed by Tucker, states that quantum limited responsivity is in fact predicted with then non-linearity is sufficiently sharp, defined as:

$$
\left[I_{d c}\left(V_{b}+\frac{h \nu}{e}\right)-I_{d c}\left(V_{b}\right)\right] \gg\left[I_{d c}\left(V_{b}\right)-I_{d c}\left(V_{b}-\frac{h \nu}{e}\right)\right]
$$

where $I_{d c}$ is the current response, and $V_{b}$ is the bias voltage [16]. Due to the high non-linearity and quantum nature of an SIS tunnel junction, quantum effects must be taken into account when (2.10) is valid. A computer simulation by Tucker has shown that unlike a classically resistive mixer, when quantum effects are taken into account, infinite conversion gain is possible [17]; a prediction subsequently verified experimentally by Kerr [18]. SIS based mixers, with greater than unity conversion gain, have been shown to produce noise temperatures approaching the quantum limit, $\hbar \omega / k[19]$. For Nb based SIS junctions, voltage scales are on the order of $\mathrm{mV}$, making high-quality SIS junctions with sharp non-linearities suitable mixing elements for quantum limited heterodyne $\mathrm{THz}$ receivers. 


\subsection{Requirements for Higher Frequency Operation}

With the development of higher frequency SIS based mixers, constraints are imposed through the substrate, fabrication techniques, and material properties of both the superconducting films and tunnel barrier. Three main considerations for higher frequency operation are discussed below and addressed in subsequent chapters as part of this work.

\subsubsection{Substrate Requirements}

As frequencies enter the THz range, the detection wavelengths enter the sub-millimeter and micron regime, increased reduction in wavelength and device geometries push the limits of standard fabrication techniques and processes. The use of Silicon-OnInsulator (SOI) technology in conjunction with novel fabrication techniques, first developed by our research group, allows the realization of ultra-thin chips only a few microns in thicknesses with Au beamleads [20]. The few-micron thick substrates substrates offer many unique benefits such as: reduced RF loss through the increased cut-off frequency of the substrate modes, the ability to define final chip geometries through photo-lithographic and RIE etching techniques, and the ability to include Au beam-leads which extend beyond chip geometries allowing for easy handling and electrical connections. Examples of such chips are shown in Figure 2.6.

Despite these unique benefits, it is important to note that one potential drawback of this technology is that moderate film stresses can cause ultra-thin chips to deform and bend, making devices impossible to mount in flat test fixtures, such as commonly used split-block waveguides. The thin-Si process was originally developed for use on SOI substrates without a thermal $\mathrm{SiO}_{2}$ device layer. However, for a series of ultra-thin mixers fabricated at UVML that required a thermal $\mathrm{SiO}_{2}$ device layer, it was noticed that the individual devices would curl and bend when released from their carrier due 

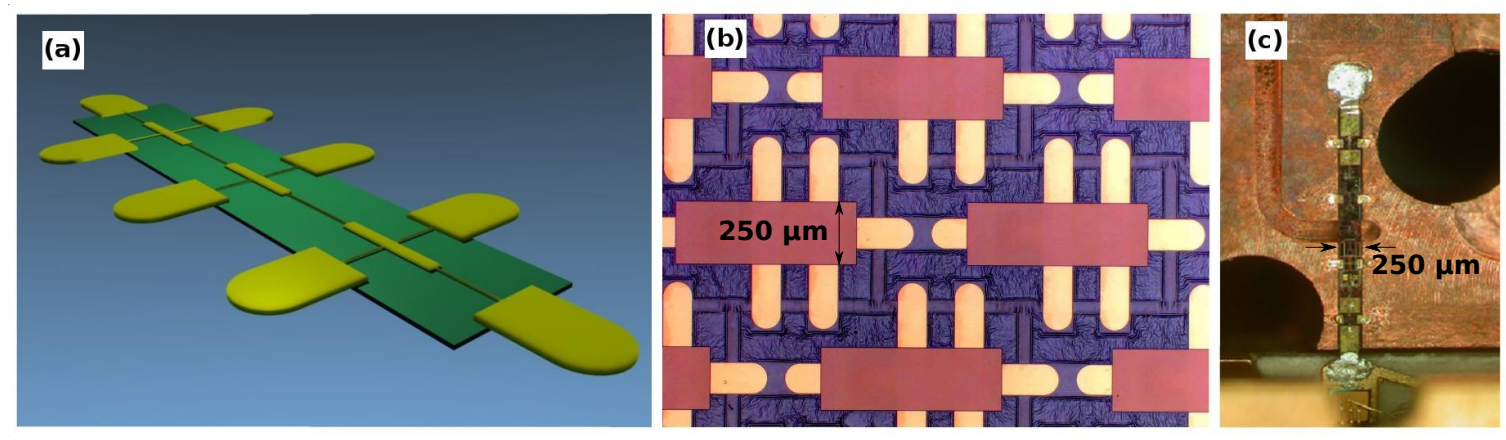

Figure 2.6: (a) Computer model of generic thin-Si ship demonstrating top-side circuitry and gold beam leads which extend past Si-chip geometries. (b) Micrograph of 0.5 THz mixer circuits mounted to a Si carrier, demonstrating the efficient packing resulting from lithographic and RIE etch-based chip definition. (c) Micrograph of a UVML fabricated mixer mounted inside a split-block waveguide.

to the stress of the $\mathrm{SiO}_{2}$ films. My initial research at UVML developed a processcompatible stress compensation technique to alleviate these issues and produce nearly flat chips.

The developed method consists of depositing a stress compensation layer on the backside of the thin-Si substrate. Diode sputtered $\mathrm{SiO}_{2}$ was chosen as it is process compatible and sputtering is a room-temperature process that allows precise control of internal film stress through deposition conditions. In order to calculate the required thickness and stress of the compensation film, one determines the curvatures induced in the devices as a result of film stress. This can be done by direct optical measurements of bent devices or calculated from the stress of a film deposited using similar growth conditions. Stoney's equation relates the internal stress of a thin film on a rectangular substrate to the measured deflection and material properties as:

$$
\sigma_{f}=\frac{Y_{s} d_{2}^{2}}{6 R\left(1-\nu_{s}\right) d_{f}}
$$

where where $Y_{s}$ and $\nu_{s}$ are Young's modulus and the Poisson ratio of the substrate, $R$ is the measured radius of curvature, and $d_{s}$ and $d_{f}$ are the thicknesses of the substrate and film. The proper thickness of the stress compensation layer is determined 
by rearranging (2.11) as follows:

$$
d_{f}=\frac{Y_{s} d_{2}^{2}}{6 R\left(1-\nu_{s}\right) \sigma_{f}}
$$

The compensation film is then deposited on the backside of the thin-Si. A comparison between a properly stress compensated chip and an uncompensated chip is shown in Figure 2.7. This process has been successfully implemented in current SIS mixer circuits fabricated at UVML and a more detailed explanation of the entire fabrication process is published in my previous thesis [21]. A limitation is that standard dicing and DC dip testing of individual mixers is not possible, as the wafer must remain whole during the thinning process for thin-Si technology. For previous mixers fabricated by our research group on quartz wafers, such as those for ALMA Band 6, POGO test elements (further described in Chapter 4) are diced from the carrier wafer and the IVs of the mixer circuits are verified through submersion in liquid helium and DC testing before the time consuming dicing and thinning of individual mixers. However, for thin-Si mixer technology, the individual mixers cannot be screened and tested until the thinning process is completed and individual devices are mounted, a process that can take in excess of month. We do note that in addition to thin-Si mixer chips, larger multi-device multi-beamlead thin-Si test chips are included on the wafer. However, mounting of the multi-device test chips is also a time consuming process in which the test chip must be placed into a fixture, electrical connects made with silver epoxy to the individual beamleads, and cannot be performed before the time consuming backside process. An optimal solution would be the ability to both: (a) DC test individual devices before the backside thinning process, and (b) DC test individual chips after the backside process to make sure the backside process hasn't affected the IVs. Screening individual mixers may be particularly important for balanced mixer implementations and multi-pixel arrays, where more than one well matched mixer is required. With this in mind, an initial investigation of the potential use of a modified 


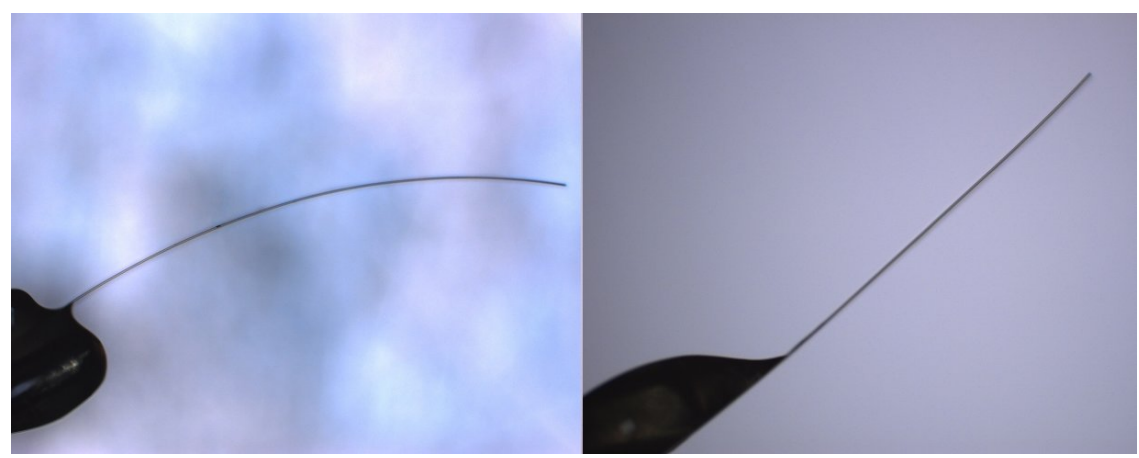

Figure 2.7: Left: Edge on view of $3 \mu \mathrm{m}$ thick $\mathrm{Si} / \mathrm{SiO}_{2}$ chip without stress compensation. Right: Same geometry chip with proper stress compensation.

commercially available cryogenic probing station, to test entire device wafers before the lengthy backside thinning process, is invested as part of this work in Appendix I.

\subsubsection{Superconducting Material Requirements}

While the optimal bias point for SIS heterodyne mixers is often $V_{b} \approx V_{g a p}-\frac{h \nu}{2 e}$, and they can theoretically perform as heterodyne mixing elements at frequencies up to twice the gap frequency, $\nu_{\text {gap }}=\frac{E_{\text {gap }}}{h}$, the performance of all $\mathrm{Nb}$ based mixers will be significantly degraded above the gap frequency. The increased loss at this point is a result of both: (a) Josephson-effect noise as the LO oscillations cause the bias point to swing past 0 VDC forming a hysteretic loop on each RF cycle and (b) ohmic loss in the superconducting transmission lines (wiring layers) as photons above the gap frequency break Cooper pairs. The Josephson-effect noise can be reduced through suppression of the DC Josephson current, discussed in greater depth by Tucker and Feldman [13]. Focusing on loss in the wiring layer, at $0 \mathrm{~K} \mathrm{Nb}$ has an energy gap of $2.8 \mathrm{meV}$ which corresponds to a gap frequency of $\sim 700 \mathrm{GHz}$. Low loss operation above $\sim 700 \mathrm{GHz}$ requires either the use of a low resistance normal metal or a higher bandgap superconducting material for the wiring layers, and such implementations using $\mathrm{Al}$ wiring layers have been shown extend the low noise operation of $\mathrm{Nb}$ SIS 
mixers past the gap frequency of $\mathrm{Nb}[22]$.

While $\mathrm{Al}$ transmissions lines have lower loss than $\mathrm{Nb}$ transmission lines at frequencies above $\sim 700$, a normal metal will always introduce ohmic losses, and a better suited material is a higher bandgap superconductor [23]. Upon the start of dissertation research, our research group began investigating the use of alternative higher bandgap superconducting materials such as NbTiN. NbTiN films can have a bandgap roughly $\sim 1.5-1.8$ times that of elemental Nb. The increased bandgap of NbTiN allows frequency operation up to $\sim 1.2 \mathrm{THz}$ for Nb/Al-AlN/NbTiN based SIS junctions [23]. $\mathrm{NbTiN}$ is formed via reactive sputtering of a $\mathrm{NbTi}$ target in an argon-nitrogen plasma and a method of creating repeatably high quality NbTiN films at room temperature using standard sputtering equipment has been previously developed at UVML and implemented in $\mathrm{Nb} / \mathrm{Al}-\mathrm{AlN} / \mathrm{NbTiN}$ junctions [24]. We note, that while room temperature deposition of NbTiN is attractive, the highest bandgap NbTiN films have been deposited on heated substrates. However, the RF properties of NbTiN are different than that of $\mathrm{Nb}$ when incorporated in RF mixing circuits and, if these difference are not taken into account, errors of up to $50 \%$ in design parameters may result [25].

The impedance of a superconducting microstrip transmission line differs from that of an ideal lossless line as the penetration depth affects the impedance of the superconducting microstrip line through the kinetic inductance introduced by the Cooper Pairs. The solutions for the inductance and capacitance of a superconducting microstrip line have been published by W.H. Chang as:

$$
L=\frac{\mu_{0}}{W K}\left[h+\lambda_{1}\left(\operatorname{coth}\left(\frac{t_{1}}{\lambda_{1}}\right)+\frac{2 p^{1 / 2}}{r_{b}} \operatorname{csch}\left(\frac{t_{1}}{\lambda_{1}}\right)+\lambda_{2} \operatorname{coth}\left(\frac{t_{2}}{\lambda_{2}}\right)\right)\right]
$$

and

$$
C=\frac{\epsilon \epsilon_{0} W K}{h}
$$

where $W$ is the center conductor line width, $h$ is dielectric thickness, $t_{1}, \lambda_{1}$ and $t_{2}$, 
$\lambda_{2}$ are the thickness and the penetration depth of the center conductor and ground plane respectively. The factors $K, r_{b}$ and $p$ take into account fringing fields and are determined by the microstrip geometries [26].

Techniques for measurement of penetration depth existed at the time, but either required bulk samples or dicing of thin film samples, and are incompatible with SOI processing techniques utilizing ultra-thin substrates previously mentioned [27, 28]. As part of my initial research at UVML, on-wafer test devices were developed and successfully tested, producing results which matched well with BCS theory. Specific details are beyond the scope and length of this text, but are published in my previous paper [29]. Initial results and proposed work to incorporate higher band-gap NbTiN films for both the base and counter electrode of AlN based SIS junctions are covered in Appendix II.

\subsubsection{Barrier Requirements and Junction Geometry}

An ideal tunnel barrier fully insulates the superconducting base electrode from the counter electrode by solely allowing a tunneling current to flow, producing a highly non-linear characteristic SIS IV curve. The ideal requirements for the tunnel barrier are complete coverage and low subgap leakage insulating characteristics. Additionally, the geometry of the SIS junction, as shown in Figure 4.2, is that of a parallel plate capacitor. When used in RF mixing applications, a capacitance can short out the high frequency signals if not taken into account. A capacitance can be partially tuned out with a shunted inductance, often created with a length of superconducting transmission line, but such a circuit is only optimal around one design frequency with a bandwidth, $B \propto 1 / R C$, where $C$ and $R$ are the capacitance and normal state resistance of the junction. $R$ is generally fixed for proper impedance matching, therefore in order to maximize bandwidth, one must reduce the capacitance through reduction of junction area. 
Reduction in junction area raises its own difficulties in research environments where contact alignment printing is often employed. A 5 layer 'petalevel' self-aligned junction definition process has previously been developed at UVML to produce junctions as small at $0.28 \mu \mathrm{m}$ diameter using standard I-line contact lithography [30]. While such a process is paramount for precise control of junction size during final mixer fabrication, the additional processing time can pose considerable constraints when multiple experimental samples are to be fabricated and tested. In this work, a new mask set was designed that allows a wafer to be scribed into separate pieces in order to isolate individual components of the process flow for investigation, and an 'Al quadlevel' self-aligned junction fabrication process was devloped, that finds a balance between fabrication time and control of junction size. These topics are introduced as part of this work in Chapter 4.

One must also keep in mind that if one reduces junction size, while maintaining a constant current density, $J_{c}$, the impedance of the junction will rise with decreasing area. As devices are scaled to higher frequency, the barrier thickness must be reduced in order to increase current density and maintain a reasonable impedance, while retaining a high quality insulator to block subgap leakage. Standard junction technology predominately uses Al-oxide from an Al overlayer for its simplicity of formation and high quality barrier at low current densities. However, for higher current densities, the thinner Al-oxide barriers eventually become conductive and alternative barrier materials such as AlN are required. Additionally, (2.3) demonstrates an exponential dependence of $J_{C}$, and hence junction impedance, with respect to (barrier thickness $)^{-1}$. As such, precise control of barrier thickness, in addition to quality, is paramount to the realization of SIS junctions with proper impedances for mixing applications. Modifications to the trilayer deposition system, in order to increase repeatability of AlN growth, are discussed in the next chapter. Additionally, the use of a spectroscopic ellipsometer and spectrometer to monitor aspects of the ICP ni- 
tridation of $\mathrm{Al}$ films in real time provides a greater understanding of the growth and quality of ICP grown AlN films. These topics are presented in Chapters 5 and 6 . 


\section{Chapter 3}

\section{Trilayer Deposition and Barrier Formation}

\subsection{Background of SIS Trilayer Growth}

High quality, low-leakage SIS junctions with Nb electrodes and aluminum oxide tunnel barriers, produced through the oxidation of $\mathrm{Al}$ overlayers, are widely reported in the literature and have become integral in the design and fabrication of a variety of superconducting circuits. It was first shown by Gurvitch that when a thin overlayer of $\mathrm{Al}$ is sputter deposited onto a $\mathrm{Nb}$ film and subsequently oxidized and another $\mathrm{Nb}$ layer is added to form a Nb/Al- $\mathrm{Al}_{2} \mathrm{O}_{3} / \mathrm{Nb}$ stack, the resulting SIS junctions produce excellent low-leakage IV curves for two key reasons [31] . Firstly, Al readily forms an insulating oxide in the presence of oxygen and the barrier thickness can be precisely controlled run to run through a controlled oxygen exposure. Secondly, it was found that sputter deposited $\mathrm{Al}$ 'wets' the surface of $\mathrm{Nb}$ and an $\mathrm{Al}$ overlayer thickness of only $\sim 10 \AA$ fully covers the $\mathrm{Nb}$ surface and prevents oxidation of the underlying $\mathrm{Nb}$ film [32]. The ability of an $\mathrm{Al}$ overlayer to fully cover an underlying $\mathrm{Nb}$ film and form an insulting oxide reduces subgap leakage current through the tunnel barrier, 
producing high-quality, low-leakage junctions. However, as higher current density SIS tunnel junctions are desired, the increasingly thin $\mathrm{Al}_{2} \mathrm{O}_{3}$ barriers are eventually shown to introduce excess leakage currents due to defects and pinholes in the barrier [33]. As such, alternative barrier materials are currently being investigated.

AlN is one potential alternative to $\mathrm{Al}_{2} \mathrm{O}_{3}$. It can be produced through nitridation of $\mathrm{Al}$ overlayers and hence, has Al's ability to wet the surface of $\mathrm{Nb}$. However, more energy is required to break the triple bond of $\mathrm{N}_{2}$ than the double bond of $\mathrm{O}_{2}$. As a result, $\mathrm{Al}$ does not readily react with $\mathrm{N}_{2}$ at ambient temperatures. Either high substrate temperature or the use of a nitrogen plasma is required to break the $\mathrm{N}_{2}$ bonds, allowing the formation of AlN. Various methods of AlN barrier formation have been proposed, ranging from direct barrier deposition through reactive sputtering [34] to nitridation of Al films via parallel-plate plasma [35], ion gun [36], and ICP [24].

Direct barrier deposition, through reactive sputtering, can lead to difficulty of precise barrier thickness control, as deposition rates tend to vary run to run and over the life of a target. With respect to AlN growth through nitridation, a nitrogen plasma bombards the substrate with nitrogen ions, potentially leading to film damage. AlN growth via ICP nitridation of $\mathrm{Al}$ overlayers with $\mathrm{Nb}$ base electrodes, an approach first investigated by our group at UVML, appears promising in this respect, as the ionic species have low kinetic energy. However, $\mathrm{Al}$ overlayers have been found to be incompatible with higher energy gap NbN and NbTiN base electrodes, as the nitrogen in $\mathrm{NbN}$ and $\mathrm{NbTiN}$ tends to react with the Al overlayer [37]. Therefore, the formation of AlN barriers by nitridation of an $\mathrm{Al}$ overlayer is not possible with $\mathrm{NbN}$ or NbTiN base electrodes. Initial work has been performed at UVML with respect to the use of directly sputtered AlN tunnel barriers, but the results failed to produce high quality junctions. A summary of our investigations and potential future research based on our findings is included in Appendix II. For these reasons, the ICP nitridation of an $\mathrm{Al}$ overlayer for use with $\mathrm{Nb}$ base electrodes is investigated in this work. 


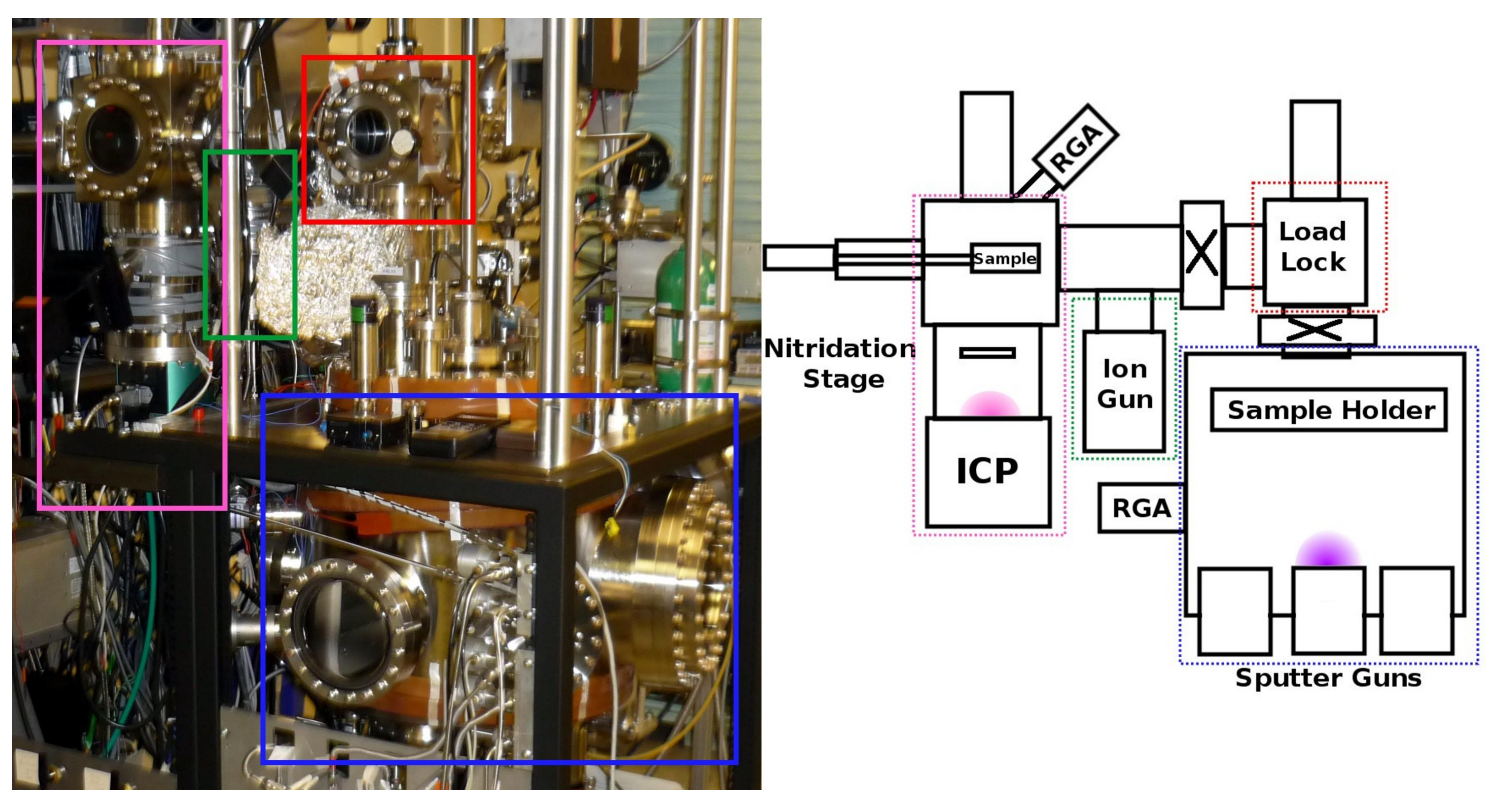

Figure 3.1: Photograph and schematic representation of the trilayer deposition system used in this work.

Previous attempts at trilayer growth utilizing ICP grown AlN barriers at UVML produced fairly low-leakage high-quality SIS junctions, however it was shown that the repeatability of the normal state resistance junction area product, $\left(R_{n} A\right)$, of successive wafers drifted over time by up to an order of magnitude [38]. The ability to predict and control junction impedance is paramount to the realization of superconducting $\mathrm{RF}$ circuits, such a drift in $R_{n} A$ is a limiting factor. This chapter will discuss modifications made to the trilayer deposition system (in order to improve the repeatability of $R_{n} A$ ) and the subsequent results for both test junctions, and the first AlN based $500 \mathrm{GHz}$ mixer on SOI realized at UVML.

\subsection{Modifications to Deposition System}

All trilayer films were deposited using a custom built high vacuum sputtering system shown in Figure 3.1, which will be referred to as "Tri-3" for the rest of this text. The system consists of two stainless steel chambers connected by a load lock. Both chambers and the load lock are separated by gate valves and pumped by Pfeiffer 
turbo-molecular pumps. The first (deposition) chamber contains four 3" target Nb, $\mathrm{NbTi}, \mathrm{Au}$, and $\mathrm{Al} \mathrm{DC}$ magnetron sputtering guns with a target to wafer separation of $80 \mathrm{~mm}$. Situated above the guns is a water-cooled, stepper motor controlled, rotating platter designed to hold and position the samples during deposition. The second (nitridation) chamber contains an MPS 3000 ion gun and Copra DN160 ICP source, with a source to target separation of $80 \mathrm{~mm}$ and $91 \mathrm{~mm}$ respectively. For vacuum level analysis, both the deposition and nitridation chambers contain SRS RGA-100 residual gas analyzers (RGA).

The Tri-3 system show in Figure 3.1, was modified from the original trilayer deposition system used in the aforementioned ICP AlN based SIS work by Cecil [39]. The specific modifications will be discussed in detail in the following subsections.

\subsubsection{Reconfiguration of the ICP source and Addition of an Ion Gun}

The original ICP source used in Tri-3 to first realize ICP grown AlN tunnel barrier was model 160-E from CCR Technologies, and it is worth noting that it contained proprietary circuitry that allowed the ion energy to be controlled independently of the ion current density at low pressures $(0.2-2 \mathrm{mT})$ through an 'energy knob' located on the unit [40]. Following our lead, researchers at Delft Institute of Technology purchased a similar ICP unit from CCR Technologies and began growing Nb-AlN trilayer using an identical process. The unit was similar in all respects to ours, except it did not contain the energy control circuitry. Delft reported repeatable $R_{n} A$ results, contrasting our variation of $R_{n} A$ over time [41].

The details of this circuitry are proprietary and, as such, were not disclosed by CCR. From our observations of the ICP unit, the energy knob was mechanically connected to a series of variable parallel plate capacitors and it is suspected additional energy is capacitively coupled to the plasma. While the unit was designed to affect ion 
energy only at pressures below our operating point of $\sim 5-20 \mathrm{mT}$, the position of the energy knob was shown to significantly affect AlN growth rates within this pressure range, possibly through variation of plasma species further discussed in Chapter 6 [39].

Specific modifications to the ICP unit included the removal of the energy control circuitry. Removal of the circuitry was not trivial and was a several month long process that required sending the unit to CCR Technologies for a complete overall by their team of engineers. We make note that after the ICP unit underwent significant modifications by CCR Technologies, and was reinstalled in our deposition system, the initial trilayer was of poor quality, producing short circuited junctions. Process parameters were varied and all aspects of fabrication were heavily scrutinized for nearly a year to no avail - no increase in IV quality was measured. It was not until the ICP unit was removed and dissembled by our group that we noticed a protective quartz cap, which isolates an exposed stainless steel gas inlet from the plasma, was not installed and the protective quartz liner was covered in a redeposited conductive dark material, as shown in Figure 3.2.

It is important to note that the original design of Tri-3 also relied on the ICP unit for in situ sample cleaning through use of a low pressure $(0.3 \mathrm{mT})$ high power (500 W) Ar plasma. The high energy Ar ions sputter etched away the 5-10 nm of the substrate surface, providing a clean surface for trilayer deposition. It is believed sputter etching of the stainless steel gas inlet during in situ Ar ICP cleaning was the source of the dark contamination. It is postulated that such conductive contamination could cause conductive shorts through our thin barrier layers. Furthermore, minute Fe contamination can significantly reduce the superconductivity of the surface layer on our Nb films, also destroying SIS behavior [42].

In order to resolve these issues, the contaminated quartz liner was cleaned in both strong acids with oxidizing agents and then buffered oxide etchant to remove visi- 

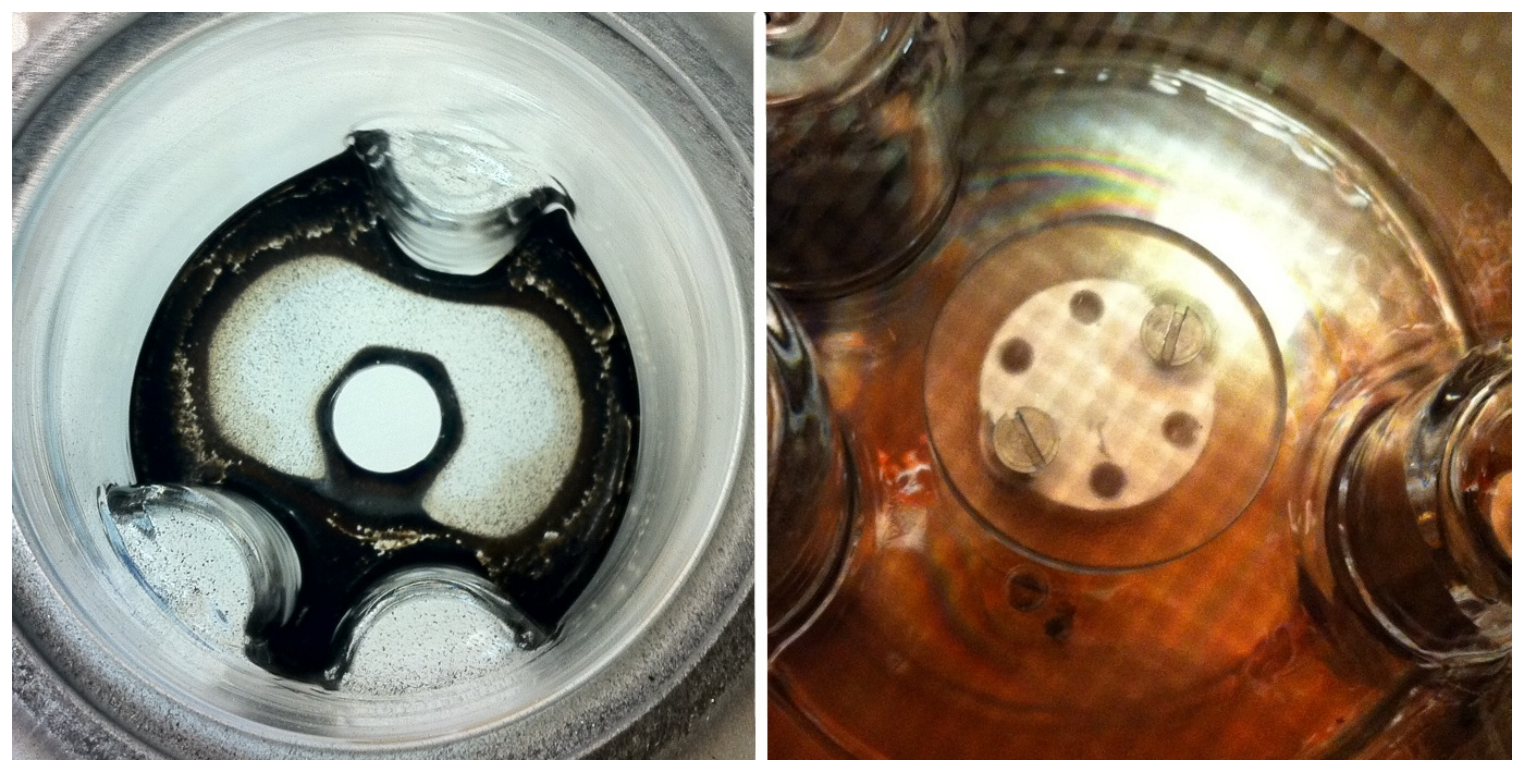

Figure 3.2: Left: Quartz liner removed from ICP unit after stainless steel gas inlet was exposed to a low-pressure high-power Ar plasma. Right: New quartz liner, with stainless steel post removed and replaced with $\mathrm{Al}$ post, as well as the addition of a quartz protective cap fastened with $\mathrm{Al}$ screws.

ble contamination while a replacement liner was being manufactured. The missing quartz cap was obtained from CCR Technologies and all steel parts were replaced with aluminum alternatives, as shown in the right side of Figure 3.2. After these modifications, the IV characteristics of our junctions improved over time, which is attributed to the slow removal of residual contamination from the system. Resolution of this issue was paramount to further progress, as short circuited junctions are unsuitable for heterodyne mixing and had brought our research to a halt.

To further help eliminate the possibility of contamination due to in situ cleaning, an ion gun was installed between the load-lock and nitridation chamber to allow in situ cleaning of our samples away from critical components and dedicate the ICP to nitrogen plasma chemistries needed for AlN growth. Periodic inspection of the ICP unit revealed no visible contamination from this point forward. 


\subsubsection{Isolation of Load Lock and Addition of RGA and El- lipsometer}

In addition to the aforementioned changes made to the ICP source, the original system design required the entire nitridation chamber to be vented while loading samples. Lack of repeatability was conceivably due in part to the extra circuitry of the ICP source as well as contamination from frequent venting. During the period when the ICP unit was reconfigured by CCR Technologies, in-house modifications of the deposition system took place. More specific details, including part numbers and CAD drawings, can be found in Appendix III. Modifications included a redesign to allow the addition of dedicated turbo pump to the nitridation chamber and a gate valve to isolate it from the load lock during venting, as shown in Figure 3.1. The RGA allows for monitoring of background gases and along with the use of a spectroscopic ellipsometer, aimed at the sample when situated above the ICP (which was installed before modifications began and further discussed in Chapter 5), allowed recording of background oxidation of a freshly deposited metal film.

Figure 3.3 compares the unintended ambient background oxidation of a freshly sputtered Al film before and after all aforementioned system modifications. For each test, a new $\mathrm{Si} / \mathrm{SiO}_{2}$ wafer was loaded and an optically thick $\mathrm{Al}$ film was sputtered onto the surface. The wafer was then immediately transferred to the nitridation chamber and the oxidation was monitored in situ using a J.A. Woollam M-2000® Ellipsometer. A baseline measurement was taken for two cases before the system modifications, one while flowing $99.9999 \%$ purity nitrogen gas to achieve a pressure of $5 \mathrm{mT}$ and the other under high vacuum. While nitrogen was flowing, an over 4 fold increase in oxidation rate was observed. Due to the high purity of the nitrogen used, and that it is thermodynamically impossible to produce AlN at ambient temperature with diatomic nitrogen, we believe the observed oxidation is a result of increased desorption of water molecules from chamber walls from the nitrogen flow. After the 
system modifications, using the newly installed RGA, the background partial pressure of $\mathrm{H}_{2} \mathrm{O}$ was recorded before each run. Figure 3.3 shows the background oxidation of a freshly deposited $\mathrm{Al}$ film under $5 \mathrm{mT}$ of nitrogen with the partial pressure of water varying from 3e-9 to 1e-8 Torr, as well as a baseline taken (blue line) under high vacuum. We note a reduced oxidation rate at $5 \mathrm{mT}$ nitrogen pressure is observed for lower $\mathrm{H}_{2} \mathrm{O}$ partial pressures and when the partial pressure reaches a value of $\sim 3 \mathrm{e}-9$ Torr, no oxidation is observed.

A typical sample with a freshly deposited $\mathrm{Al}$ overlayer sits in a nitrogen background for a time on the order of minutes before the plasma is ignited and AlN growth begins. As previously discussed in Chapter 2, tunneling current is exponentially proportional to (barrier thickness) ${ }^{-1}$. It is argued that unintended (or initial) background oxidation may affect final total barrier thickness as well as junction quality through the formation of suboxides and may also contribute to the observed variation of $R_{n} A$ over time before the system modifications.

\subsection{SIS Trilayer Growth and RnA Results}

SIS tunnel junctions were fabricated and DC tested at cryogenic temperatures to test both junction quality and the repeatability of $R_{n} A$. After proper nitridation conditions were chosen, mixers designed to operate at the 385-500 GHz 'Band-8' frequency band of the ALMA radio telescope were fabricated on $3 \mu \mathrm{m}$ SOI wafers producing the chips shown in Figure 2.6 and RF tested at operating frequency at NRAO facilities in Charlottesville, VA.

All trilayer for DC test samples was deposited on $330 \mu \mathrm{m}$ thick, $50 \mathrm{~mm}$ diameter DSP Si wafers with 1500-2500 $\AA$ of thermally grown $\mathrm{SiO}_{2}$. The trilayer for Band-8 wafers was deposited on SOI wafers with a $3 \mu \mathrm{m}$ thick device layer, $1 \mu \mathrm{m}$ thick buried oxide layer, and a $600 \mu \mathrm{m}$ thick handle layer. All wafers were cleaned in situ prior to 
Parameters vs. Time

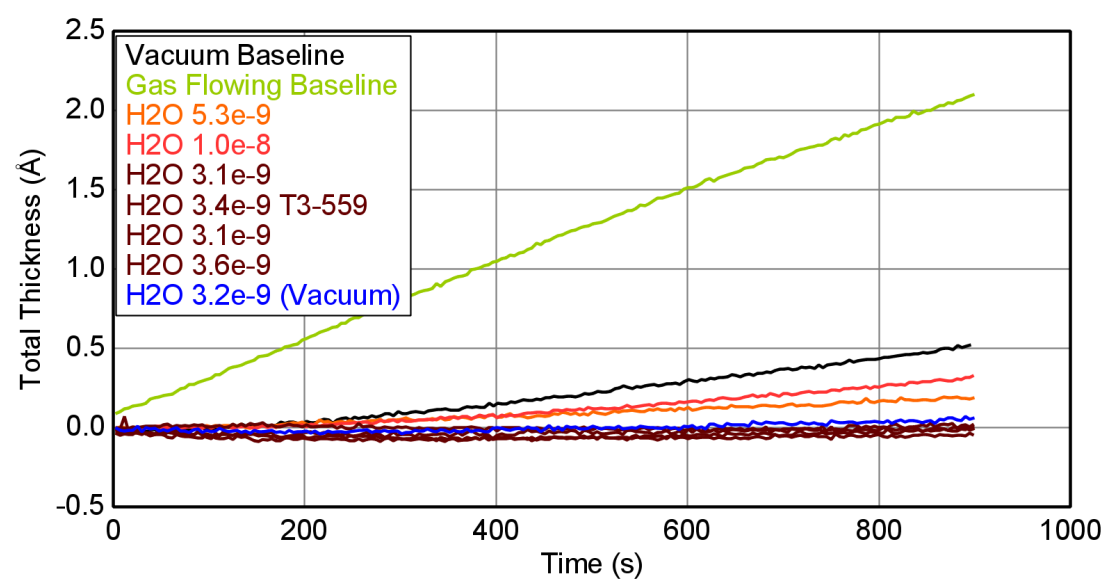

Figure 3.3: Oxidation of $\mathrm{Al}$ as monitored by a J.A. Woollam M-2000® Ellipsometer. The two 'baseline' measurements occurred before any system modifications, the black line is the background oxidation of $\mathrm{Al}$ under vacuum, and the green line is the background oxidation under $5 \mathrm{mT}$ of nitrogen. The rest of the measurements occurred after system modifications, where the partial pressure of $\mathrm{H}_{2} \mathrm{O}$ is expressed in Torr. The orange and red lines are measurements taken under $5 \mathrm{mT}$ of nitrogen, and the blue line is taken under vacuum for comparison. At around 3e-9 Torr, there is no observed background oxidation. All pressure units are in Torr.

deposition via Ar ion milling and both chambers were allowed to reach a base pressure in the $10^{-8}$ Torr range with a partial pressure of water in the $10^{-9}$ Torr range. Nb films were deposited by DC magnetron sputtering in Ar with an applied power of 180 $\mathrm{W}$ and at a pressure of 5.0-6.5 mT. For AlN barriers produced via ICP nitridation, a 5-7 nm thick Al overlayer was deposited via DC magnetron sputtering at $205 \mathrm{~W}$ at $6.0 \mathrm{mT}$ and subsequently exposed to an ICP nitrogen plasma at $5.0 \mathrm{mT}$ with applied power of $200 \mathrm{~W}$.

Using the aforementioned deposition processes, $\mathrm{Nb} / \mathrm{Al}-\mathrm{AlN} / \mathrm{Nb}$ trilayer was produced and junctions were fabricated using a self-aligned negative photoresist process. Electrical connections were made to the junctions with sputter-deposited Nb/Au contacts and DC testing was performed at $4.2 \mathrm{~K}$ by submersion in liquid helium.

IV characteristic of a contamination-free $\mathrm{Nb} / \mathrm{Al}-\mathrm{AlN}(\mathrm{ICP}) / \mathrm{Nb}$ tunnel junction with $J_{c}=4 \mathrm{kA} / \mathrm{cm}^{2}$ is shown of the left of Figure 3.4. The plot demonstrates 

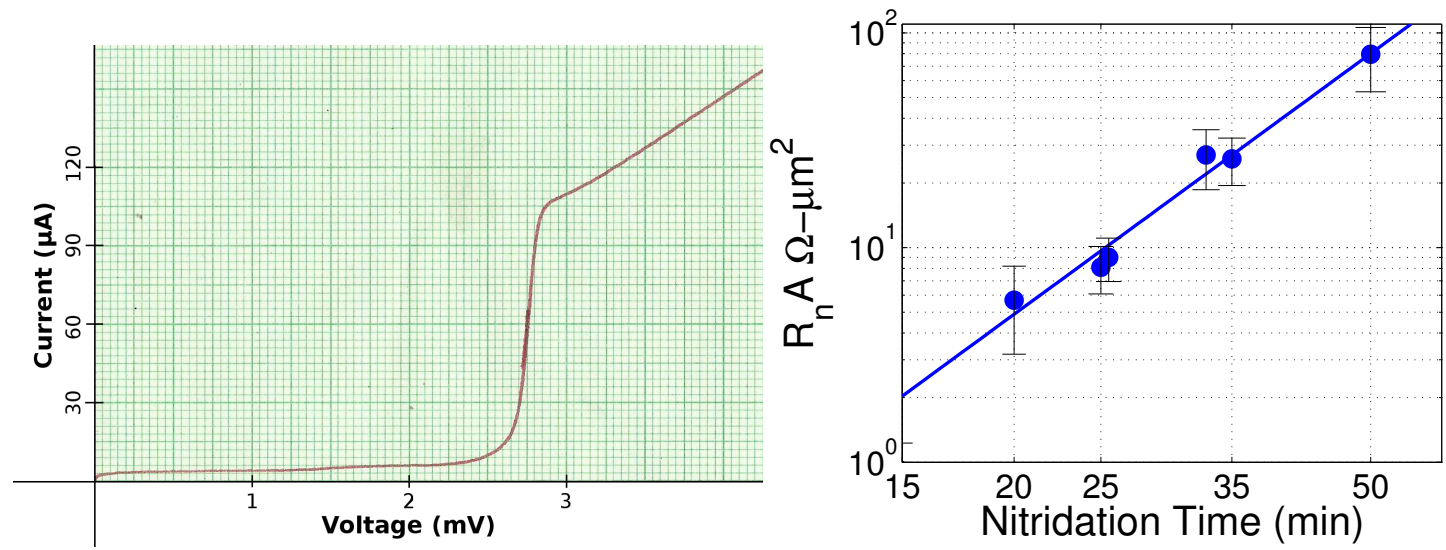

Figure 3.4: Left: IV characteristics of an AlN based SIS junction for the work presented in this chapter $\left(J_{c}\right.$ of $4 \mathrm{kA} / \mathrm{cm}^{2}, 1.4 \mu \mathrm{m}$ diameter $)$. Right: $R_{N} A$ with respect to nitridation time for a series of test runs.

fairly low subgap current and sharp non-linearity at sum-gap voltage. Additional junctions were fabricated with varying nitridation times in order to investigate the dependence of $R_{n} A$ with nitridation time for use with Band-8 mixer fabrication. The results are shown in right side of Figure 3.4. The results show reasonable fit of $R_{n} A$ vs nitridation time to a straight line in log-log space. This demonstrates a significant improvement, as $R_{n} A$ would vary by an order of magnitude before system modifications and for a given SIS mixer design a specific $R_{n} A$ value is required for proper impedance matching. It was not feasible to properly design and fabricate AIN based SIS mixers before these system modifications allowed the growth of AlN trilayer with predictable $R_{n} A$ and proper SIS IV characteristics.

\subsection{ALMA Band-8 Results}

As shown in Figure 3.4, ICP nitridation of a thin Al overlayer produced SIS trilayer suitable for heterodyne mixer applications and, with our modified ICP unit and deposition system, demonstrated a predictable $R_{n} A$ across multiple runs. This method of AlN growth was used to produce trilayer and a batch of ALMA Band-8 heterodyne mixers were fabricated. The ALMA telescope covers the frequency range of 31-950 
$\mathrm{GHz}$, broken up into 10 frequency bands. Our research group at UVML has successfully fabricated Al-oxide based mixers for Band-3 (84-116 GHz), Band-6 (211-275 GHz), and Band-7 (275-370 GHz) that met ALMA requirements, but before this work began, we had not yet demonstrated mixers for Band-8 (385-500 GHz). The ALMA specifications for Band- 8 require a receiver noise temperature of less than 4 times the

photon temperature, $T_{q}=\frac{h \nu}{k}$, over $80 \%$ of the band, and NRAO's mixer design specified a $J_{C}$ of $20 \mathrm{kA} / \mathrm{cm}^{2}$ demonstrating the need of high quality low-leakage AlN-based SIS junctions [43].

\subsubsection{Post Fabrication Device Damage}

The initial batch of Band- 8 mixers, were first tested at room temperature, and produced junction impedances roughly in agreement with design specifications. The chips shown in Figure 2.6, were initially mounted to a silicon carrier wafer during room temperature testing, and dismounted prior to shipping to the NRAO facilities for cryogenic testing. Upon testing a few weeks later, the engineers at NRAO noticed an increased room-temperature resistance, with many mixers producing open circuit characteristics.

In further investigation, we tested additional mixers that remained stored at UVML. The stored mixers remained mounted to the carrier and produced similar impedances as when initially tested weeks prior. Additionally mixers were dismounted and retested at room temperature; a rise in impedance was observed with a few mixers producing open circuit characteristics. Noting this, it is hypothesized that the increased resistance and open circuits are a result of ESD damage incurred while dismounting the chips from the carrier wafer, or afterwards during storage. Chips were initially dismounted onto a piece of filter paper and stored in a non-conductive plastic Petri dish. To eliminate the possible influence of static charges, additional chips were carefully dismounted onto an metal-woven conductive ESD wiper and stored 

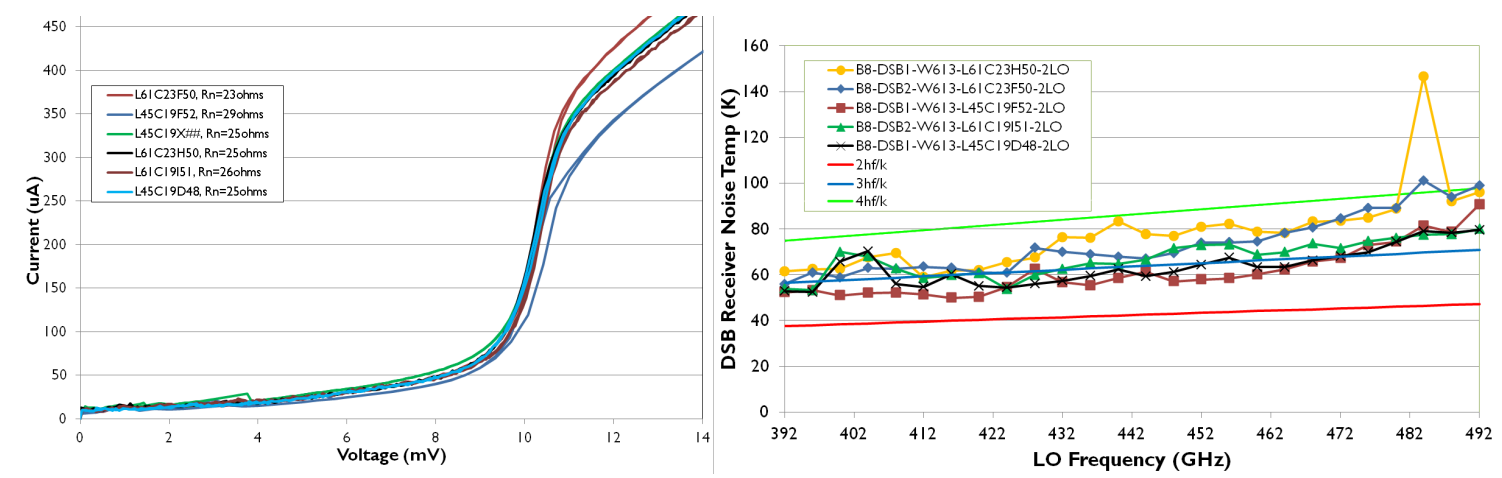

Figure 3.5: Left: IV Characteristics of Band-8 mixers. Right: Resulting noise temperature measurements.

in a grounded Al-coated Petre dish. Samples were stored and shipped in ESD safe containers and grounding straps were worn while handling. The resulting chips produced characteristic IV's and good noise temperature results as shown in Figure 3.5. This observation is worth noting, as the fabrication and testing laboratories are now equipped with ionizing air blowers to neutralize static charges and wafers are now stored in conductive ESD reducing petre dishes to prevent similar device damage.

\subsubsection{Noise Temperature and IV results}

Standard Y-factor noise temperature measurements were performed at the NRAO Central Development Laboratory in Charlottesville, VA. The resulting IV curves and corresponding noise temperature measurements are shown in Figure 3.5. The majority of mixers demonstrated consistent IV characteristics and a noise temperature below 4 times the quantum limit, meeting ALMA Band- 8 specifications. We note the $J_{c}$ of the junctions was calculated to be $\sim 35 \mathrm{kA} / \mathrm{cm}^{2}$, instead of the mixer design specification of $20 \mathrm{kA} / \mathrm{cm}^{2}$. Due to a miscommunication, a specification of 30 $\mathrm{kA} / \mathrm{cm}^{2}$ was incorrectly assumed and used to determine the ICP nitridation time. Despite this error, the resulting mixers produced good results, and demonstrated our groups ability to reasonably control $R_{N} A$ through varying nitridation time. 


\subsection{Conclusions}

When I began my research at UVML, our research group had previously demonstrated the ability to create prototype devices on thin-Si substrates with Au beamleads, as well as the ability to produce AlN SIS junctions with good IV characteristics. However, there were unforeseen issues which arose, preventing the ability to realize SIS mixers utilizing this technology. Unlike previous thin-Si HEB based mixers that were fabricated on bare Si SOI substrates, SIS devices require a thermal oxide grown on the Si surface, which has been observed to both reduce Nb film stress and reduce etching of the substrate during trilayer RIE. When our research group fabricated our first series of aluminum oxide based SIS mixers on $3 \mu m$ thick silicon substrates, the intrinsic stress in the thermal oxide caused chips to curl and bend. Such bending makes chips unsuitable for mounting into flat fixtures. A stress management technique described in Chapter 2 resolved these issues allowing the incorporation of thin-Si technology with SIS mixers.

However, as our research group moved toward ICP-grown AlN based trilayer, despite demonstrating good IV characteristics, the repeatability of $R_{n} A$ varied by an order of magnitude over time. SIS mixers are designed around a specific $R_{n} A$ value and cannot be realized with such run to run variation. It is worth noting that we were the first research group to use an ICP source to grow AlN trilayer and it was not fully understood what parameters affected AlN film growth. It was believed that the proprietary energy control circuitry, unique to our ICP unit, affected AlN film growth, possibly through variation of the relative species present in the plasma. A major redesign of our deposition system was undertaken, which involved the reconfiguration of the ICP unit and the addition of a spectroscopic ellipsometer, which is discussed in Chapter 5. Initial AlN trilayer grown after the modifications was of unusable quality, and is believed to be the fault of engineers at CCR Technology who forgot to install a protective quartz cap, exposing stainless steel parts, which subsequently 
contaminated the system. After the ICP unit was cleaned and properly configured, IV characteristics began to improve, and run to run repeatability of $J_{c} / R_{n} A$ was significantly increased. The ability to realize AlN based SIS junctions and mixers sets the groundwork for the research discussed in the rest of this dissertation. We do note the Band-8 mixer IV characteristics in Figure 3.5 contain an appreciable amount of leakage current and the $J_{c}$ of our trilayer was higher than design specification. Noting this, there is room for improvement, particularly in reducing subgap leakage. An investigation using spectroscopic analysis of the nitrogen ICP, in order to find optimal ICP growth conditions, is discussed as part of this work. Additionally, as new nitridation conditions, and potential improvements to the fabrication process were investigated, multiple test wafers needed to be fabricated and tested. A New Test mask set and SIS junction fabrication process, to reduce overall fabrication time for test devices, is covered in the following chapter. 


\section{Chapter 4}

\section{Al Quadlevel Junction Fabrication and SIS Test Mask}

\subsection{Introduction}

In Chapter 3, we discussed the modifications made to our trilevel deposition system and noted that we slowly lost the ability to realize high quality SIS junctions. Contemporaneous to these modifications, the graduate student who first realized ICP grown AlN based trilayer had left our research group, processing equipment was changed in the lab, and, due to our system being down for modification, months passed before we began producing AlN trilayers and fabricating AlN based SIS junctions. The source of the decreased quality of our SIS junctions was not initially apparent, forcing our research group to scrutinize every aspect of our fabrication process. Evaluation of the effect of variation of our process parameters required the full fabrication of a single wafer for every iteration. Noting the number of possible variations to our process, and the complexity of our current junction definition process, over a year passed before we discovered the cause of our problems was due to the ICP unit having been incorrectly configured by CCR Technologies and it was missing a critical component. Spend- 
ing over a year fabricating and testing SIS junctions for troubleshooting purposes demonstrated the need for a more rapid fabrication process, which still mimicked our current "pentalevel" process, to realize micron and sub-micron SIS junctions for testing purposes. A time efficient Al Quadlevel Process was developed in this work, and is discussed in detail in this chapter.

In addition to a rapid fabrication process, our current "Test Mask" set, used to fabricate SIS test junctions, required the entire wafer to remain whole during the process. As we varied process parameters, each iteration required a separate trilayer sample. In addition to time constraints from multiple fabrication runs, variations may exist from wafer to wafer, through quality of the starting trilayer or variation that may occur in any one of the processing steps, adding ambiguity as a non-direct comparison. Noting this, a new Test Mask set, designed to be scribed into four standalone quarters during any part of the fabrication process, was developed. Additional test elements, designed to troubleshoot common failure mechanisms, are also included and provide further insight to individual aspects of fabrication. The development and novel features of the mask set are discussed in the second half of this chapter.

As we discuss the development of the $\mathrm{Al}$ quadlevel junction fabrication process and the new SIS Test Mask, the following nomenclature will be used for brevity: 'M1' refers to the base superconducting layer of the SIS trilayer stack, 'M2' refers to the top superconducting layer of the SIS stack, capped with Au for this work, and 'M3' refers to the superconducting wiring layer that makes electrical connections to the 'M2' junction electrodes and is capped with Au for test devices, as shown in Figure 4.1. 


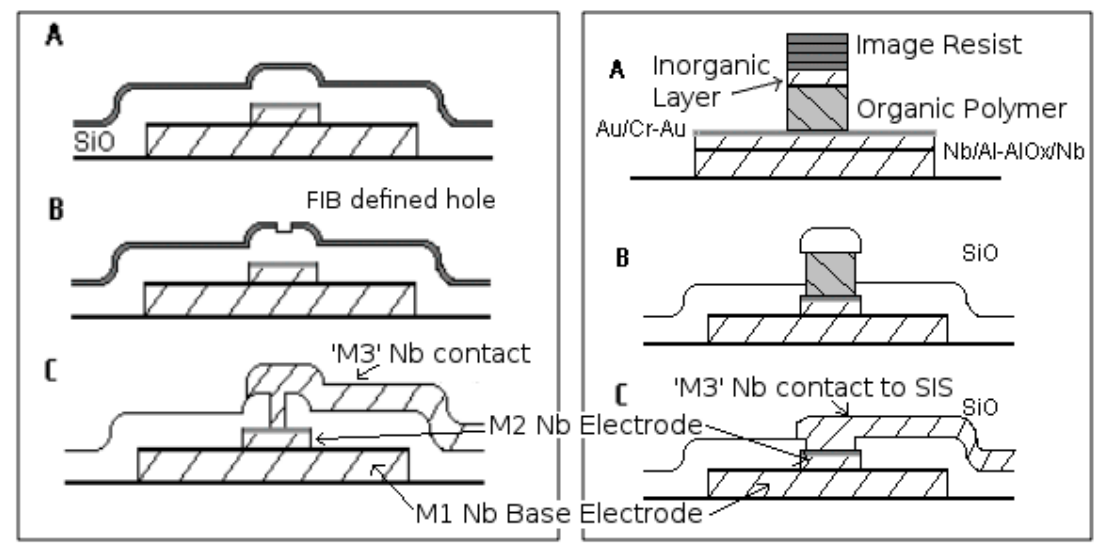

Figure 4.1: Left: Machine aligned scheme, previously realized by our research group, a hole in the $\mathrm{SiO}$ insulation is opened with the use of a FIB to pattern a $\mathrm{Cr}$ etch mask. Right: Self-aligned organic/inorganic stack. An image resist patterns the inorganic layer, which serves as an etch mask for the underlying polymer. The entire stack defines the top electrode by means of RIE and serves as a liftoff mask for the subsequent junction insulation deposition [44].

\subsubsection{Background of Modern SIS Junction Fabrication}

The strong non-linearity and low subgap leakage of high-quality SIS junctions enable them to function as quantum-limited heterodyne mixing elements. The starting trilayer material must contain a high quality tunnel barrier, to prevent subgap leakage, and be grown by means of a repeatable process, to achieve desired current densities. The junction impedance is proportional to its (diameter squared) ${ }^{-1}$, and, with dimensions on the order of one micron, accurate process control is essential for properly matched RF devices. In addition to accurate definition of junction size, the wiring layers, which both feed DC bias and RF signals to the junction, must be fully insulated from the rest of the circuitry, else they create short circuits in parallel with the SIS junctions. As a result, not only must the starting SIS trilayer material be of high quality, but so must the process of physically defining and insulating the micron to sub-micron junctions. Realization of the junctions and their insulation is typically the most challenging aspect of fabrication. There are two main approaches to defining and insulating SIS junctions: the machine-aligned or self-aligned techniques. 
Using machine-aligned techniques, the junction is first defined via some form of photolithography and RIE etching. The photoresist is removed and the insulating layer is deposited on the entire device. However, to access the top electrode of the junction, the device must either be patterned again to open a via through the insulating layer by means of etching techniques, or lapped down to the M2 layer through a chemicalmechanical planarization (CMP) process [45]. Machine-aligned techniques decouple the junction definition and insulation steps, allowing the use of a thin high-resolution photoresist to precisely define the junction dimensions, but may introduce increased complexity to the fabrication process. A difficulty with CMP techniques is the accurate planarization and removal of the 100-300 nm thick insulation layer, stopping once the M2 layer is exposed; our group has explored patterning and etching of vias in the insulation layer for machine-aligned techniques. However, with junction diameters on the order of a micron, the definition of vias through the insulation requires the use of either focused ion beam (FIB) or electron beam patterning techniques with sub-micron alignment capabilities [44]. Such techniques can be time consuming, often on a one junction at a time basis, and not readily available at our research laboratory, making machine-aligned techniques at UVML currently impracticable.

Self-aligned techniques rely on the use of a single feature which acts as both an etch mask for junction definition, and remains in place to serve as a liftoff pattern for the subsequent junction insulation. The single feature can be a simple single photoresist layer, however, to achieve an aspect ratio suitable for liftoff, precise control of junction size suffers. To overcome this limitation, a stack of 1-2 $\mu m$ thick organic polymer 'planerization' layer(s) is often employed. The stack is covered by a thin, on the order of $100 \mathrm{~nm}$, inorganic 'masking' layer(s). The thin inorganic layer(s) can be precisely defined through use of a high resolution photoresist and standard etching techniques. The inorganic layer(s), which are not affected by the oxygen plasma used to define the organic layer(s), serve as an etch mask. The result is a feature with a 
proper profile for liftoff. A general comparison between two machine and self-aligned techniques is shown in Figure 4.1.

Our group's original implementation of self-aligned junction definition process (the first implementation of a multi-resist process for SIS junction fabrication) relied on the use of a 1-2 $\mu \mathrm{m}$ thick polyimide layer capped with a $140 \mathrm{~nm}$ thick $\mathrm{SiO}_{2}$ layer, and patterned with standard photoresist [46]. The $\mathrm{SiO}_{2}$ layer is etched using a $\mathrm{CF}_{4}+\mathrm{O}_{2}$ plasma at 50 mTorr pressure, stopping on the polyimide surface. The polyimide is etched by an $\mathrm{O}_{2}$ plasma, providing a columnar polyimide/ $/ \mathrm{SiO}_{2}$ structure similar to that shown in Figure 4.1. The $\mathrm{Nb}$ is etched with a similar $C F_{4}+O_{2}$ plasma, but at higher (300 mTorr) pressure. The high pressure etch was found to be very isotropic, undercutting the polyimide/ $/ \mathrm{SiO}_{2}$ structure, and the subsequent insulation would not reach, and hence not fully seal, the junction. To better seal the $\mathrm{Nb}$ junction, the polyimide is shrunk after the $\mathrm{Nb}$ RIE, exposing the perimeter of the junction and allowing full $\mathrm{SiO}_{2}$ coverage. However, the fluorine used in the $\mathrm{Nb}$ etch hardened the outside perimeter of the polyimide, making the shrinking process unpredictable, and also often spread debris that had to be carefully removed with a hydrofluoric acid based wet etch. While this process produced high quality junctions, the control over junction size is relatively poor due to the isotropic $\mathrm{Nb}$ etch and undercutting. This process was also limited to $\sim 2 \mu m$ sized junctions, due to the isotropic $\mathrm{Nb}$ etch and the long polyimide shrink times required to insure that the fluoride hardened polyimide shell was removed. It was later observed that a switch to a $S F_{6}+N_{2}$ based RIE significantly increased the anisotropy of the $\mathrm{Nb}$ etch, but yield was still affected due to the unpredictability of shrinking the fluorine hardened polyimide layer [47].

The next signification improvement to our self-aligned process came in 2003 through the use of a 'Ti quadlevel' stack. In addition to the use of a $S F_{6}+N_{2}$ based RIE to define the $\mathrm{Nb}$ electrode, it was found that the use of JSR Micro's NFR chemically amplified Novalak resin-based chemistry significantly increased the anisotropy of the 
$\mathrm{Nb}$ junction etch [48]. For this reason, the single polyimide layer was replaced with a bilayer of NFR (thinned with methyl-3-methoxy propionate to achieve a thickness of $100 \mathrm{~nm}$ ) and polyimide. The NFR-enhanced anisotropic Nb etch removed the need for unpredictable shrinking of the polyimide layer. We also note, an additionally benefit is NFR is not readily removed with the solvents used during liftoff. Liftoff is still achieved through the dissolution of the polyimide layer, however the NFR remains behind, protecting the junction until the NFR is removed via oxygen plasma. An additional limitation of the original trilevel structure is that the $\mathrm{SiO}_{2}$ masking layer is readily etched in the fluorine based $\mathrm{Nb}$ junction etch chemistry, requiring the use of a relatively thick layer. $\mathrm{Cr}$, having excellent selectivity to $\mathrm{Nb}$, was initially tried, but lacking chlorine based RIE equipment at the time, the Cr etch mask was defined with a wet etch, limiting feasible junction size to around $2 \mu \mathrm{m}$. The masking material eventually chosen for the quadlevel structure was $\mathrm{Ti}$. Ti is readily anisotropically etched in a $F 12+C F_{4}+O_{2}$ plasma, and while it is also etched in the $S F_{6}+N_{2}$ Nb RIE, the etch rate is about one half that of Nb. The result of the NFR/polyimide/Ti/NFR(imaging) quadlevel structure was the ability to precisely fabricate junctions down to $1.5 \mu \mathrm{m}$ on a regular basis with variation in junction impedance across a $30 \mathrm{~mm}$ wafer of less than $10 \%$.

We also note that the 'Ti quadlevel' process is compatible with an Au overlayer process pursued by our research group. In the Au overlayer process, the top of the SIS trilayer is capped with 20-30 nm of Au in situ during deposition. The Au overlayer serves to prevent oxidation of the M2 Nb layer, and allows direct contact of the M3 Nb wiring layer to the top M2 $\mathrm{Nb}$ SIS electrode without the need to remove the surface oxide by means of in situ ion milling before M3 deposition. However, this adds an additional etch before the $\mathrm{Nb}$ junction RIE. Our research group first pursued the use of an $\mathrm{Au}$ wet etch, which worked well for junctions down to around $\sim 2 \mu \mathrm{m}$, but was found to cause undercutting and liftoff of smaller junction features. Noting this, it 
was found that an Ar RIE performed at a high bias of - $700 \mathrm{~V}$ and lower pressure of 20 $\mathrm{mT}$ in our SEMIGROUP RIE was able to anisotropically remove the Au overlayer.

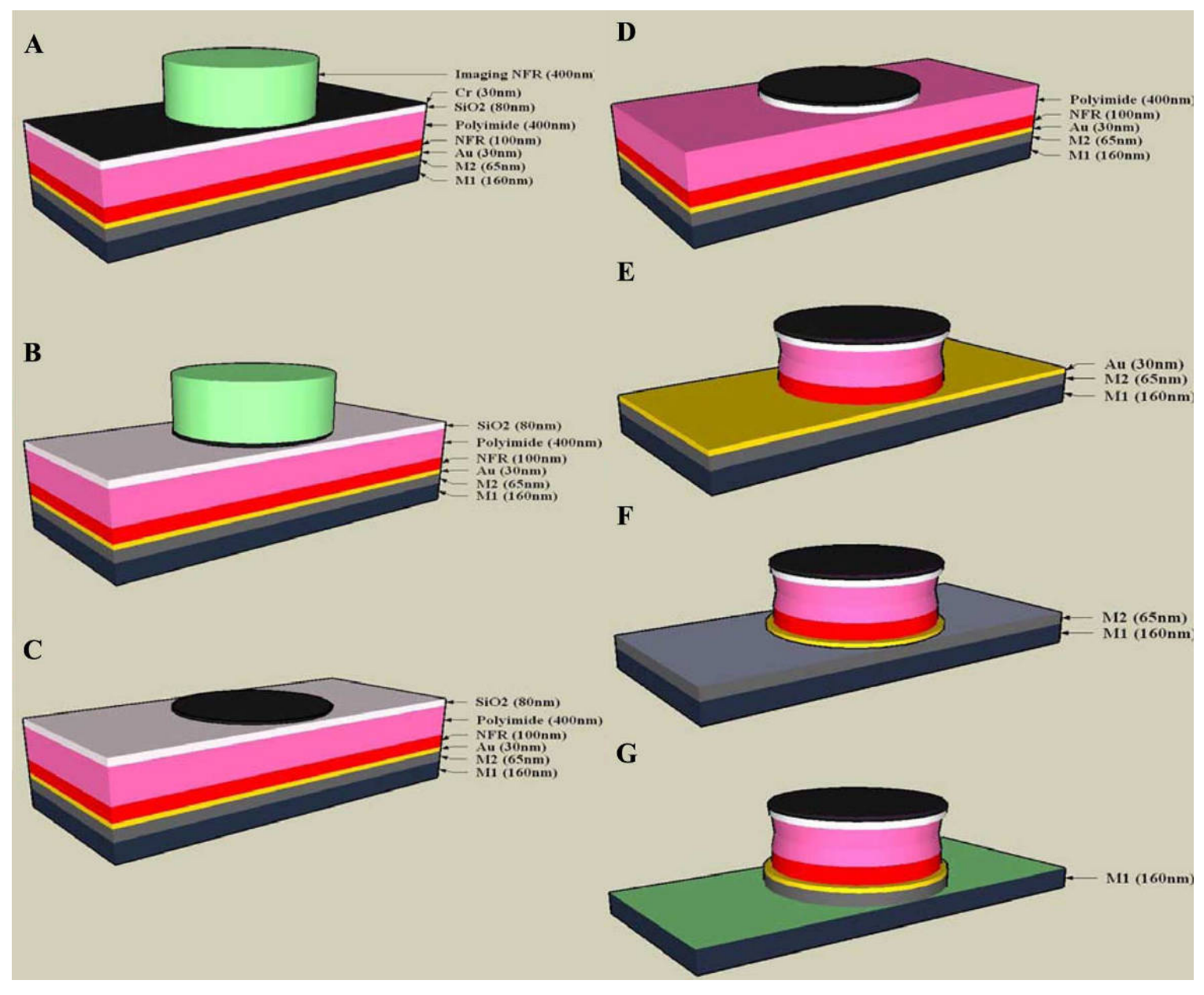

Figure 4.2: Pentalevel junction fabrication process using an NFR/polyimide $/ \mathrm{SiO}_{2} / \mathrm{Cr} / \mathrm{NFR}$ (imaging) stack. A: Stack is patterned via NFR negative resist. $\mathrm{B}$ : $\mathrm{Cr}$ is etched in a $\mathrm{Cl}_{2}+\mathrm{O}_{2}$ chemistry. $\mathrm{C}$ : $\mathrm{Cl}$ hardened imaging resist is removed via low pressure $\mathrm{O}_{2}$ plasma. $\mathrm{D}: \mathrm{SiO}_{2}$ layer is etched via $\mathrm{Ar}+\mathrm{CHF}_{2}$ chemistry. E: Polyimide is etched via $\mathrm{O}_{2}$ plasma. F: Au capping layer is etched via Ar RIE, following the pattern of the top Cr masking layer. G: Exposed Nb is etched, following the profile of the Au capping layer [30].

The most recent improvement to our self-aligned process came when current mixer designs, notably those used in the ALMA band-8 project, required the precise realization of junctions $1.0 \mu \mathrm{m}$ and smaller. While the Ti quadlevel process routinely produced junctions down to around $1.5 \mu \mathrm{m}$, a limiting factor is that the Ti mask etches, albeit slowly, during the $\mathrm{Nb}$ definition process. Around this same time, our 
lab purchased an Oxford Plasmalab-100 ICP RIE, which contained both single beam laser interferometry for in situ etch rate monitoring/endpoint detection and the ability to use $\mathrm{Cl}_{2}$ and $\mathrm{BCl}_{3}$ based chemistries [49]. The use of a $\mathrm{Cr}$ etch mask was revisited, as it readily etches in chlorine based chemistry, in development of the 'pentalevel' resist process shown in Figure 4.2. Owing to its high selectivity to fluorine based $\mathrm{Nb}$ RIE, a thin Cr layer of only $30 \mathrm{~nm}$ is sufficient to withstand the $\mathrm{Nb}$ junction etch. The $\mathrm{Cr}$ is removed via $\mathrm{Cl}_{2}+\mathrm{O}_{2}$ based $\mathrm{RIE}$, and the underlying $\mathrm{SiO}_{2}$ layer acts as both an etch stop, and adds physical robustness to the Cr layer, which is found to crack when deposited directly on the polyimide. Additionally, $\mathrm{SiO}_{2}$ protecting the underlying polyimide, allows for the removal of the chlorinated imaging resist with a low pressure $O_{2}$ plasma. After removal of the chlorinated imaging resist, the $\mathrm{SiO}_{2}$ layer is removed with an $\mathrm{Ar}+\mathrm{CHF}_{3} \mathrm{RIE}$. The NFR/polyimide stack is etched with an $\mathrm{O}_{2}$ plasma at $-50 \mathrm{C}$, and the exposed Au capping layer is then removed via $\mathrm{Ar}$ RIE in a SEMIGROUP RIE at a high bias of $-700 \mathrm{~V}$ and low pressure of $20 \mathrm{mT}$ as previously described. The high bias and low pressure allows the etch to follow the $\mathrm{Cr}$ mask at the top of the stack, and the subsequent $\mathrm{Nb}$ etch is closely masked by the Au capping layer.

The pentalevel process produces excellent quality junctions with precise control of junction size, and was used to fabricated the Band- 8 mixers shown in Chapter 3 which contained $1.0 \mu \mathrm{m}$ junctions and an impedance spread across a $50 \mathrm{~mm}$ wafer of less than $7 \%$. While this process is excellent for realization of deliverable mixers, a limitation is that it often requires more than a month of total fabrication time. As we vary processing parameters, superconductor/barrier materials, and different junction insulation schemes, many iterations are often required and the use of the pentalevel process is not feasible. A more rapid fabrication process that reduces junction fabrication time at the cost of accuracy in junction size, while closely resembling the pentalevel structure is desired and investigated as part of this work. 
In addition to reducing fabrication time for testing purposes, it is often desired to isolate specific components of the fabrication process. A new "Test Mask" set for the fabrication of SIS test elements in conjunction with the aforementioned Al quadlevel fabrication process was developed. A novel design feature of the mask set is the 50 mm wafer can be scribed into four standalone samples at any part of the fabrication process, isolating a single fabrication step, and reducing fabrication time by up to a factor of four. Further details of the test structures and key features are discussed below.

\subsection{Al Quadlevel Junction Fabrication}

As we pursued a rapid junction fabrication scheme for prototyping junctions and varying processing parameters, a single resist process was first investigated with the use of nLof negative photoresist. While suitable for initial tests of uncharacterized trilayer, there are drawbacks in terms of junction quality and control of junction sizes [50]. The standard process flow for the single resist junction scheme is shown on the left of Figure 4.3. The top electrode is defined with nLof negative photoresist and standard UV lithography. The single layer resist cannot withstand the pentalevel's anisotropic Ar based RIE to remove the Au layer; a 45 degree ion mill etch is used instead, removing Au down to the base of the resist profile. This exposes the edge of the $\mathrm{Nb}$ electrode during the subsequent $\mathrm{Nb} \mathrm{RIE}$, potentially leading to a ring of poor quality $\mathrm{Nb}$ surrounding the junction. The junctions are also not uniform in size since a much thicker imaging resist, compared to the high resolution photoresist used in the pentalevel process, is required to perform the self-aligned $\mathrm{SiO}_{2}$ liftoff. The proposed solution is to use an NFR/polyimide/Al/NFR(image) stack, the choice of materials and processing steps are discussed in this section. 

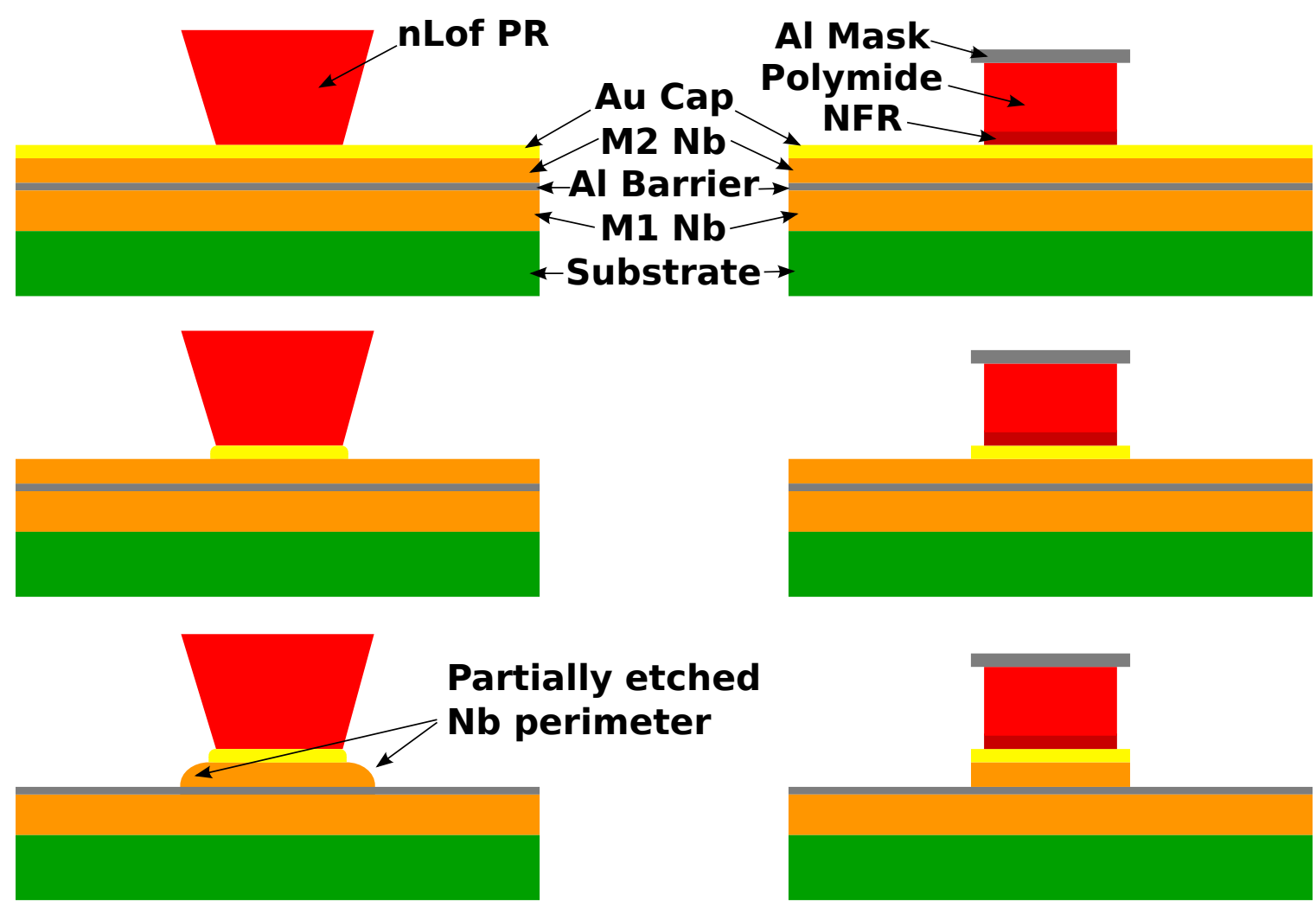

Figure 4.3: Left: Single resist junction process. The Au layer is etched via 45 degree ion mill, exposing the edges of the $\mathrm{Nb}$ electrode during $\mathrm{Nb}$ junction RIE. Right: New quadlayer process. An $\mathrm{Al}$ etch mask is used to define the polyimide/NFR stack because it can withstand a high power anisotropic Au etch as well as the subsequent $\mathrm{Nb}$ junction RIE. Due to the high anisotropy of the Au RIE, the Au etch follows the top of the NFR/poly/Al stack - the remaining Au button protects the underlying $\mathrm{Nb}$ during subsequent $\mathrm{Nb} \mathrm{RIE}$.

\subsubsection{Polymers and Al Cap}

The etch mask used in the junction process defines the geometry of the junction and must withstand all the etches of the junction definition process. Al was decided as a potentially suitable material as it is readily etched by many off the shelf wet etchants, which do not affect the underlying polyimide, is robust enough to withstand the highbias anisotropic Ar-based Au plasma etches, and it is not chemically etched by the $S F_{6}$ RIE used to define the top Nb electrode of the SIS junction [51, 52]. Al deposition by means of RF diode sputtering of an aluminum target was chosen as the method of 
deposition, as it is a room temperature deposition, producing dense films with small grain structures. Electron beam evaporated aluminum was initially investigated, but the higher substrate temperatures affected the underlying polyimide, and the grain structure of our evaporated films was on the order of our junction sizes, affecting the ability to precisely etch sub-micron features. DC magnetron sputtering was also briefly investigated, but the films were found not to etch as well as the RF sputtered equivalents.

\subsubsection{Aluminum Etching}

The aluminum was patterned using standard photolithographic processes with a high resolution NFR negative photoresist, similar to the pentalevel process. However, etching of the $\mathrm{Al}$ etch mask is not as straight forward a process. While dry RIE etches provide excellent isotropy, $\mathrm{Al}$ only readily etches in $\mathrm{Cl}$ based chemistries, which are often corrosive and incompatible with most polymers, forming chlorinated debris. $\mathrm{Al}$ readily etches in a number of wet chemistries, however, a limitation of wet etching is that it is inherently isotropic, and results in undercutting of the resist features. The $\mathrm{Al}$ etch mask defines the diameter of the junction that in turn defines the junction impedance for a given $R_{N} A$ value. A concern with regards to using a wet etch to define micron and even sub-micron features, is run to run repeatability of the amount of undercut, and the consistency across the wafer. With these concerns in mind, various dry and wet $\mathrm{Al}$ etch chemistries were pursued: $\mathrm{AZ} ® 400 \mathrm{~K}$ photoresist developer, chlorine RIE, and phosphoric acid based Transene Al etchants. The AZ® $400 \mathrm{~K}$ photoresist developer was found to lift off micron sized resist features, and is therefore incompatible. For these reasons, the use of $400 \mathrm{~K}$ as an aluminum etchant was not pursued further.

The use of an anisotropic dry chemical etch would be ideal for defining micron and sub-micron etch masks, and is utilized in the aforementioned pentalevel process 
to define a $\mathrm{Cr}$ etch mask. The use of a $B C l_{3}+C l_{2}$ RIE chemistry was explored to investigate the definition of the aluminum cap after photolithographic patterning. As shown in Figure 4.4, the resulting surface contained large of amounts of debris. Unlike the pentalevel process, which uses a layer of $\mathrm{SiO}_{2}$ underneath the $\mathrm{Cr}$, the $\mathrm{Al}$ is deposited directly on the polyimide, exposing the polyimide to the chlorine plasma during the RIE over etch. Chlorine is known to react with polymers, forming chlorinated compounds which cannot be removed with the low-power oxygen plasma used in the polyimide/NFR etch, leaving debris. Specialized chemical strippers, designed to remove debris after chlorine RIE, do exist, but they are extremely volatile and due to safety concerns in a shared educational research environment, their use is prohibited [53]. An additional consideration with the use of a stripper to remove chlorinated debris is that the stripper must not dissolve either the Al etch mask or polyimide stack.

Lastly, the use of a chemical wet etch was pursued utilizing heated Transene Type A and Type D Al etchants. Under SEM inspections, the Type A etchant produced small debris on the polyimide surface while Type D produced a clean surface free of observable debris. A reduced etch rate is also observed for type-D (130 nm/min) compared to Type A (720 nm/min) helping undercut control, allowing the realization of micron and even sub-micron sized junction features. Type D was also tried at room temperature, in hope of reducing the etch rate further. However, the resulting etches, when not heated to 40-50 C, always resulted in surface debris. A summary of the etch results is shown in Figure 4.4. 


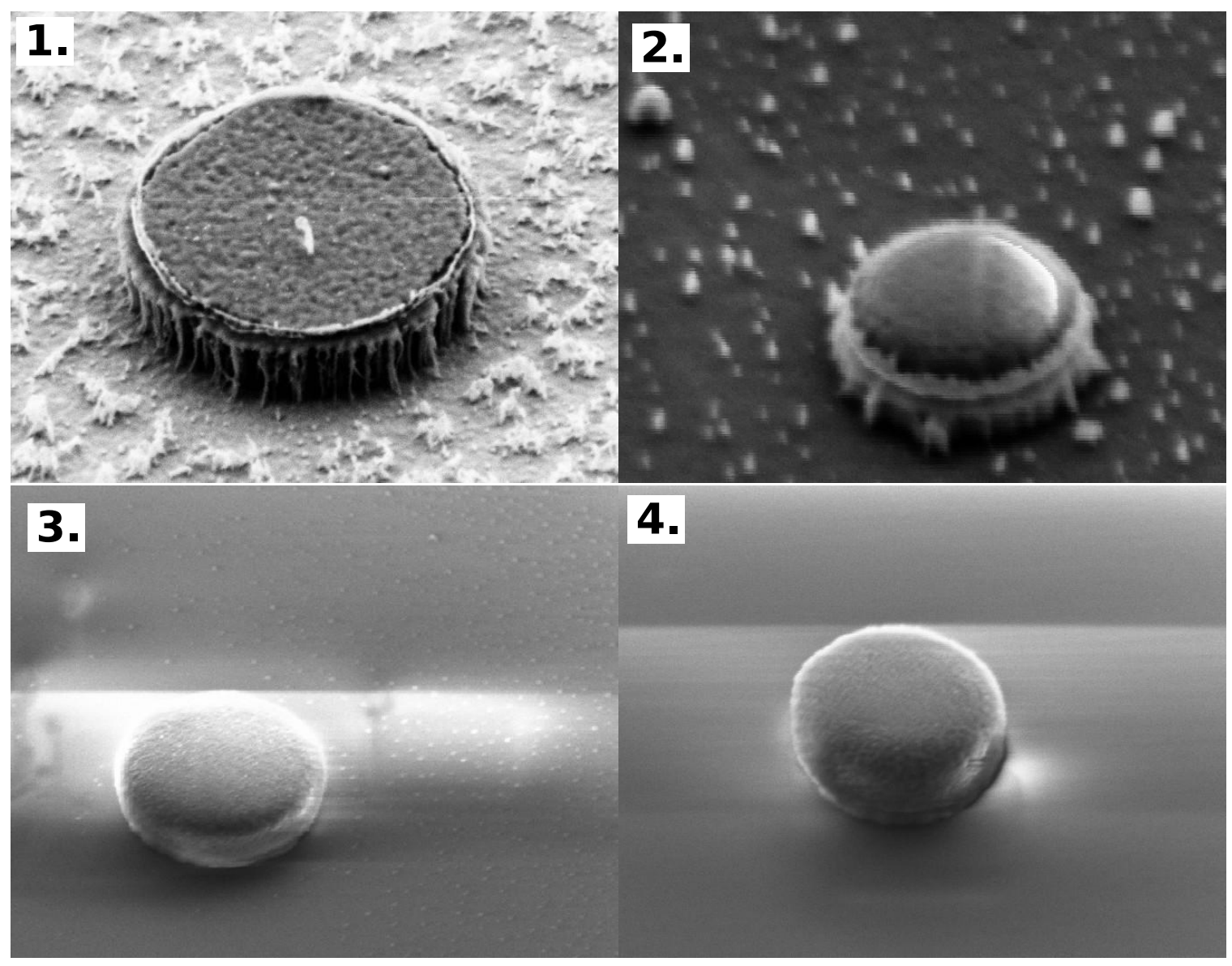

Figure 4.4: 1. Chlorine RIE etch of $\mathrm{Al}$ after subsequent polyimide RIE, debris from chlorinated polymers are present. 2. Transene Type D at room temperature. 3. Transene Type A at $50 \mathrm{C}$, produces a mostly clean etch, but some Al remains under close SEM inspection. 4. Transene Type D at $50 \mathrm{C}$, produces a clean etch. Junctions shown are between $\sim 1-2 \mu \mathrm{m}$ diameter.

\subsubsection{NFR/Polyimide Etching}

The polymers used in this work are very similar to those used in our group's pentalevel process, but it is worth noting that due to its recent treatment as a controlled substance under Schedule I of the "Controlled Substances Act", the use of gammaButyrolactone as a thinner for the polyimide had to be replaced by a less pure and potentially debris causing cyclohexane solvent when this process is employed in the United States of America. The originally developed multi-resist process utilized an NFR polyimide stack that is etched via oxygen RIE in an Oxford Plasmatherm100 
etching system with an applied RIE power of $8 \mathrm{~W}, 10 \mathrm{mT}$ of oxygen, at $-50 \mathrm{C}$. The etch consisted of 18 cycles, each containing a 5 minute etch period followed by a 2 minute "cool down" period to allow the sample to cool back to $-50 \mathrm{C}$ to increase anisotropy.

Later, during the use of the pentalevel process for device wafers, it was noted that debris periodically appeared after the polyimide etch. In response, the RF power was approximately doubled to $15 \mathrm{~W}$. The source of the debris is not clear, but is thought to be at least partially due to the switch from gamma-Butyrolacton to cyclohexane as a polyimide solvent. However, with increased RF power and increased DC self-bias, it was discovered after the NFR/polyimide etch, strands of material appeared around the junction, as shown in Figure 4.5. Such strands could interfere with the subsequent $\mathrm{Nb}$ etch, and lead to short circuits in parallel with the SIS junction.

In order to determine the source of the strands, we note that our SIS trilayer is capped with an in situ deposited layer of Au to prevent oxidation of our M2 Nb layer. Additional exposure to an oxygen plasma did not remove or reduce the strands, it was only after a short $\mathrm{Au}$ wet etch in an iodine based chemistry that the strands were removed. However, the use of a Au wet etch is not compatible with our junction definition process, as it etches the thin $\mathrm{Al}$ barrier layer at the edge of the trilayer features. It was determined that back-sputtering of the exposed Au occurs, coating the NFR/polyimide sidewalls. To reduce the back-sputtering of $\mathrm{Au}$, the applied RF power was reduced, for the $\mathrm{O}_{2}$ RIE, to $8 \mathrm{~W}$ (the minimal power able to sustain a stable plasma), and the number of etch cycles was increased to 24 , which had a similar effect of removing debris as originally increasing the power to $15 \mathrm{~W}$. Subsequent etches did not produce Au strands, as shown in Figure 4.5. 


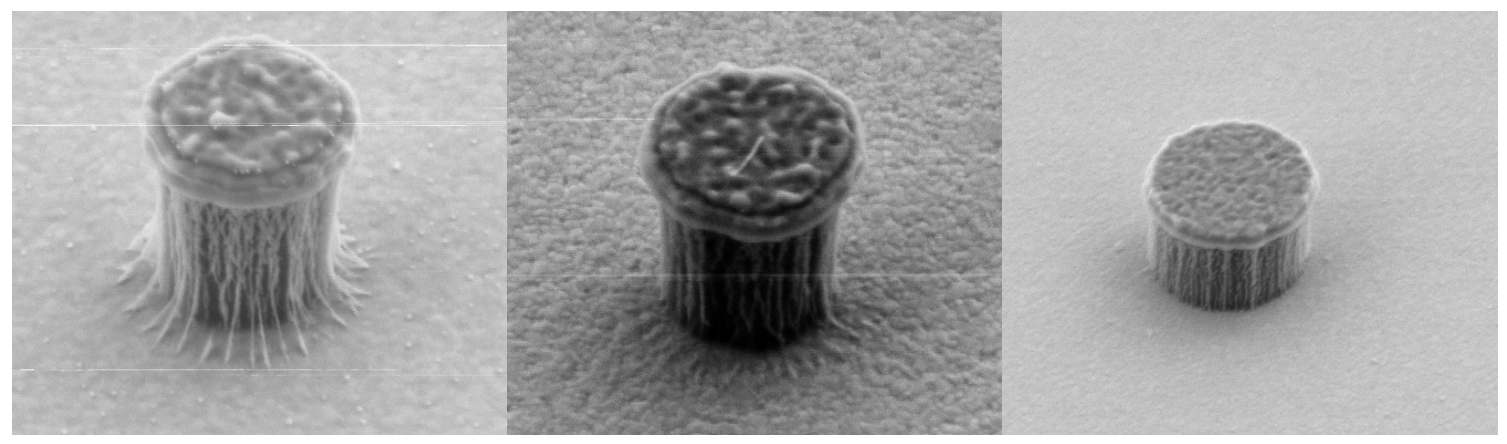

Figure 4.5: From left to right: 1 . Junction pattern ( $0.8 \mu \mathrm{m}$ diameter $)$ after NFR/polyimide etch, clearing showing strands of material. 2. Same junction as before, after an $\mathrm{Au}$ wet etch, arguing the material is back-sputtered Au. 3. Junction pattern (1.2 $\mu \mathrm{m}$ diameter) produced after reducing the applied RF power from $15 \mathrm{~W}$ to $8 \mathrm{~W}$, no visible back-sputtering of $\mathrm{Au}$ is observed.

\subsubsection{Nb/Au Top Electrode Etch and Junction Insulation}

The resulting NFR/polyimide stack, shown in Figure 4.5, is used to define the SIS junction's top electrode in the subsequent $\mathrm{Au}$ and $\mathrm{Nb}$ etches. A physical Ar RIE is used to etch the $\mathrm{Au}$ and we note that the pentalevel process utilized a separate SEMIGROUP RIE, for the Au etch. With this process, the SEMIGROUP RIE system was vented, the wafer removed, imaged via SEM, and then loaded into a separate RIE tool for the subsequent $\mathrm{Nb}$ junction etch. The resulting $\mathrm{Nb}$ etch, after being exposed to atmosphere, was found to have left debris on the barrier surface. An extensive study revisiting all steps in the pentalevel process was undertaken. Longer NFR etches, thicker $\mathrm{Cr}$ layers, short $\mathrm{Au}$ wet etches, and longer $\mathrm{Nb}$ over-etches were pursued. Short $\mathrm{Au}$ wet etches and longer $\mathrm{Nb}$ overetches, on the order of minutes, seemingly helped removed debris. However, our Au wet chemistry also attacks Al, and can damage the barrier layer. While longer $\mathrm{Nb}$ etches did help remove debris, the problem of using such a long over etch was that it would undercut the Au cap of the $\mathrm{Nb}$ junctions for non-heatsunk etches, and etch into the carrier, producing a step that may not be fully covered by the insulation layer.

In the work of this dissertation, it was observed that performing the Au etch in 
situ directly before the $\mathrm{Nb}$ etch, reduced the amount of observed debris. Additionally, if after SEM inspection the subsequent $\mathrm{Nb}$ etch was incomplete, a brief $15 \mathrm{~s}$ Ar etch to clean the substrate surface performed in situ before additional Nb etching significantly increased the ability to remove leftover Nb. For this reason, both the Ar Au etch, and subsequent $\mathrm{Nb}$ etch are performed in the same RIE, without venting in between, a notable change to the original pentalevel process.

All etches were performed in a Oxford Plasmatherm 100 ICP/RIE, the anisotropic $\mathrm{Au}$ etch was performed at $10 \mathrm{C}$, with an operating pressure of $20 \mathrm{mT}$, $50 \mathrm{SCCM}$ Ar, and an applied RF bias of $140 \mathrm{~W}$, producing a self-bias of $\sim 400 \mathrm{~V}$. The subsequent $\mathrm{Nb}$ etch was performed with an operating pressure of $10 \mathrm{mT}$, 10 SCCM Ar, 20 SCCM $S F_{6}$, and an applied RF bias of either 30 or $50 \mathrm{~W}$. An over-etch of $90 \mathrm{~s}$ is used after endpoint detection via the in-situ interferometer, slightly increased from the pentalevel process's over-etch of 30-60 s, to provide a clean surface and remove any debris present after the polyimide etch while not significantly etching into the carrier wafer. As shown in Figure 4.6, a clear undercut of the Au is shown when the longer over-etch is performed. $\mathrm{SiO}_{2}$ does not adhere well to $\mathrm{Au}$, and without exposed $\mathrm{Nb}$ around the etch of the junction, the protective ring of $\mathrm{SiO}_{2}$, which insulates the edge of the top electrode, is often lifted off during the junction insulation step, as shown in Figure 4.6, producing electrical shorts. In addition to exposing $\mathrm{Nb}$ around the junction perimeter for better adhesion of $\mathrm{SiO}_{2}$, increased anisotropy would allow for an longer over-etch time, allowing for a clean debris free surface.

There are multiple ways to increase anisotropy of an RIE. A gas such as $\mathrm{CHF}_{3}$ may be added to a fluorine base chemistry, producing radicals which form a carbonhydrogen polymer passivation layer across the substrate surface. The polymer passivation layer can be removed on horizontal surfaces through ion bombardment of RIE, but remains on the sidewalls, inhibiting undercut. While, this has been an effective and well studied method of increasing anisotropy in silicon etching for large 
area MEMs devices, the formation of passivating polymers is often affected by feature geometries, and was found not to work well with our sub-micron junction sizes. Another approach is to increase the applied RF power, raising the self-bias and increasing anisotropy. However, there exists a trade-off between anisotropy, potential substrate damage, and reduced selectivity due to physical etching/sputtering with increased ion bombardment. Another common method of increasing anisotropy, commonly used in Si etching, is through sufficiently cooling the substrate to freeze out reactive products at the surface, inhibiting lateral etching. An advantage of using freeze-out to increase anisotropy is the etch chemistry remains unchanged.

While the Oxford RIE used in this work allows for active wafer cooling via helium backing, the system is designed to etch $100 \mathrm{~mm}$ substrates, yet our devices are often fabricated on 30-55 mm substrates. When our samples are etched in the RIE, they are placed on a $100 \mathrm{~mm}$ carrier wafer where, without a thermal interface material (TIM), little heat exchange occurs under vacuum. As part of this work, in order to investigate the proper cooling of wafer samples, Apiezon L-grease was applied as a TIM and the sample was allowed to cool to $10 \mathrm{C}$ before the etch began. The result is the anisotropic etch shown in the right of Figure 4.6, compared to the etch on the left where no TIM is used. In addition, increasing the power from $30 \mathrm{~W}$ to $50 \mathrm{~W}$ was briefly investigated. However, it was found the $50 \mathrm{~W}$ etch often produced debris across the wafer. The debris is believed to be a result of micro-masking, exacerbated by both freeze-out, due to wafer cooling, and increased directionality, due to the increased self-bias at 50 W applied power. Such large debris produced nucleation points during $\mathrm{SiO}_{2}$ growth, observed as large grains visible on surface, which periodically lifted off producing pinholes visible under SEM. As a result, we settled on the use of a heat sunk $10 \mathrm{C}$, $30 \mathrm{~W}$ etch, which produced a clean anisotropic etch, and allowed for proper junction insulation. 


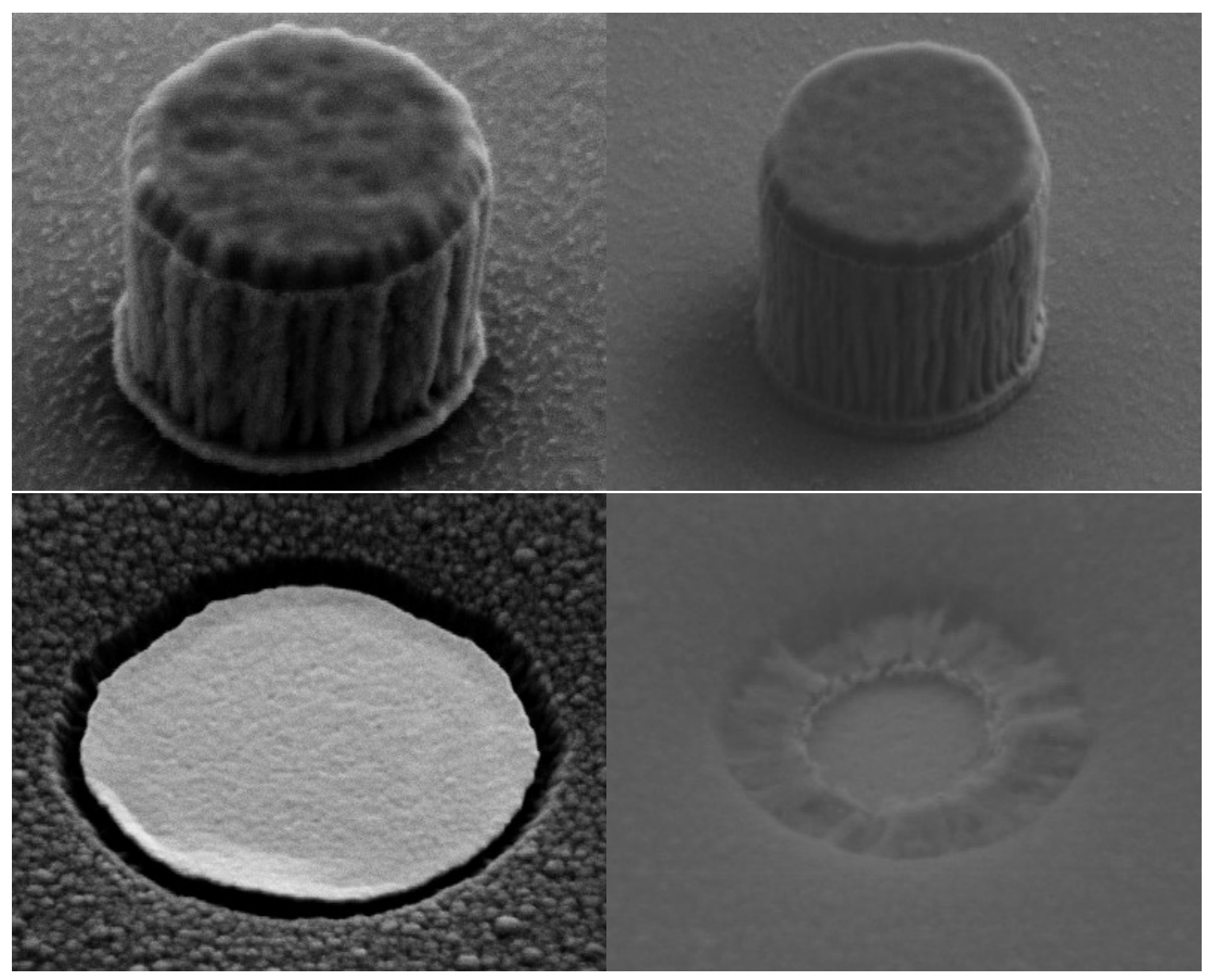

Figure 4.6: Top left: $30 \mathrm{~W}$ Nb etch without heat sinking, a clear undercut is shown. Bottom left: After $\mathrm{SiO}_{2}$ liftoff, a clear break in the junction insulation is observed. Top right: $30 \mathrm{~W}$ Nb etch with heatsinking to the carrier, Nb is clearly seen at the edge of the junction with little to no undercut. Bottom right: After $\mathrm{SiO}_{2}$ liftoff, no breaks are observed, attributed to better adhesion of the $\mathrm{SiO}_{2}$ to the $\mathrm{Nb}$ at the perimeter of the junction. Junctions shown are between $\sim 1-2 \mu$ m diameter.

\subsection{New SIS Test Mask}

The DC testing of fabricated mixer chips, such as shown in Figure 2.6, requires the mounting of individual chips into a specially designed block with silver epoxy and or crushed crushed wire compression connections. Historically, DC testing of individual test junctions was performed by making electrical connections with a West-Bond manual Au wire-bonder to Au plated contacts on a dip stick. Both methods of IV 
testing, either through DC testing of mixer circuits, or individual bonding of test junctions, are time consuming and place a practical limit to the number of junctions one may test. We note our mixer wafers often contain test elements to characterize the IV characteristics of the SIS junctions, however, the number of available test elements is limited to a few. To alleviate these constraints when we are investigating new trilayer materials or establishing new fabrication processes, our group has the capability of fabricating a separate run of SIS test devices. The final SIS test structures are diced into large $300 \mu m$ thick, 3 x 10 mm 'POGO' chips. Each chip contains 6 junctions or junction arrays, which have electrical connections to large macroscopic (2 mm x $0.8 \mathrm{~mm})$ Au coated pads. The chips are placed in a dip stick, where point contact electrical connections are made by spring loaded pins and four point IV measurements are taken (an example of the POGO test chips are shown in Figure 4.7). The use of POGO chips allows for rapid testing by submersion into a liquid helium dewar, taking minutes to cool to $4.2 \mathrm{~K}$ and warm back to room temperature. The chips are large and easily manipulated by tweezers, and because no wire-bonding is required, chips can be swapped into and out of the test setup very quickly. We also note, for this work, the use of a manual XY pen plotter to record IVs was replaced through the use of a GRAPHTEC GL900 MIDI logger and custom software written in Matlab, the details of which are provided in Appendix V. We note that it is not uncommon to test 50 or more SIS junctions in a single day using this method.

However, a limitation to the current test mask set used to produce POGO chips is that is it designed for use with a single intact $50 \mathrm{~mm}$ wafer. A major design feature of the new mask set is that it allows the wafer to be scribed into four quarters as a step of the fabrication process, by inclusion of macroscopic scribing lines in every layer, and thoughtful placement of alignment markers, as shown in Figure 4.10. In addition to the SIS junction containing POGO chips, additional test structures, shown in Figures 4.8-4.9, are included and discussed in the following subsections. 

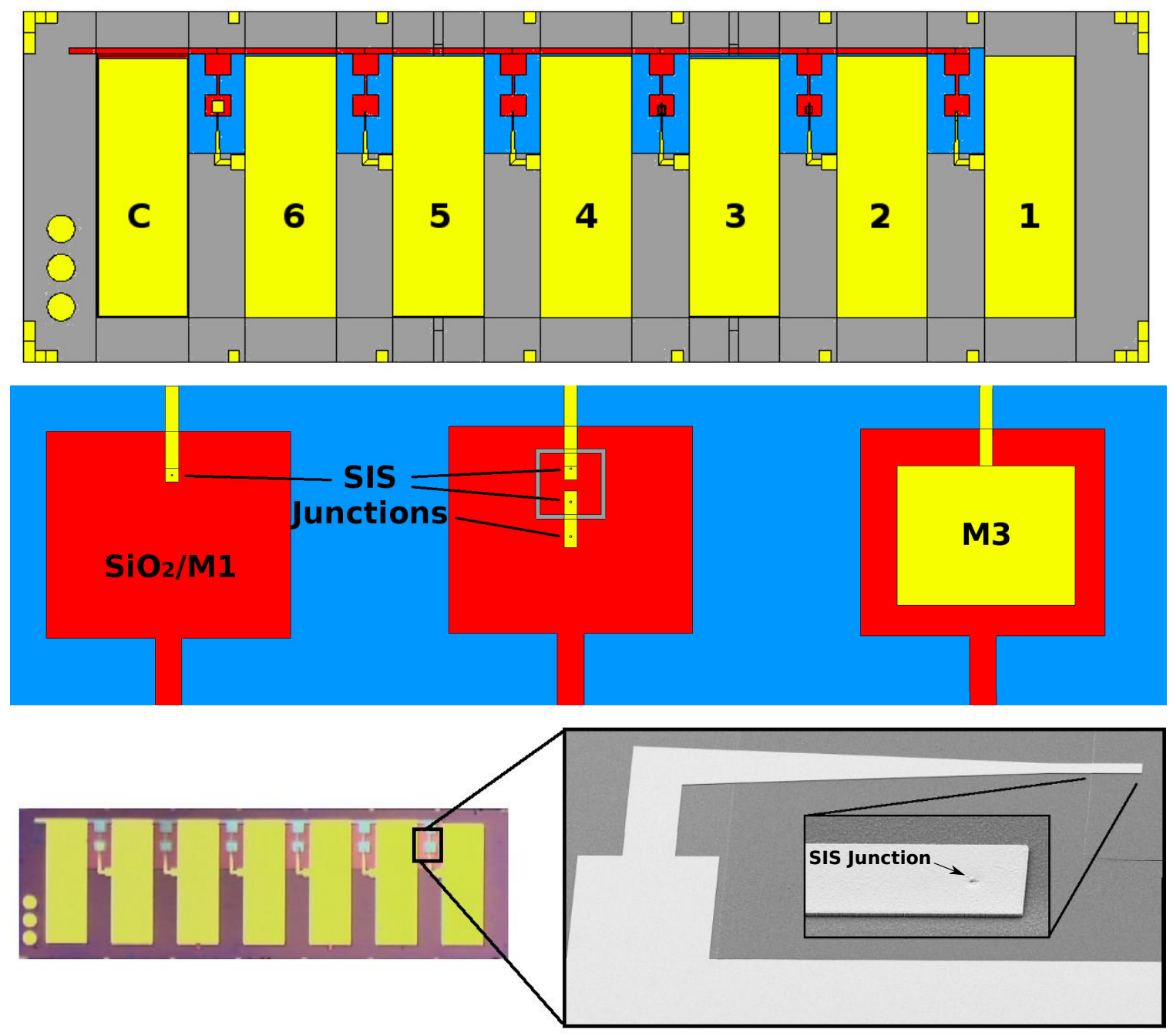

Figure 4.7: Top: CAD view of POGO chip. Pad "C" denotes the common electrode which makes connection to M1 through a large area junction. Pads 1-6 are labeled and can be independently biased via the POGO pins in our dipstick. Middle: CAD of the three available test structures on the POGO chip: a single junction element, a 3 junction series array, and an overlap with no junction to test for pinholes in the open field insulation. There are three overlap areas included: 12 x $45 \mu \mathrm{m}, 100 \times 100 \mu \mathrm{m}$, and $125 \times 160 \mu \mathrm{m}$. Bottom: Micrographs of fabricated POGO chip showing access to SIS junction. 


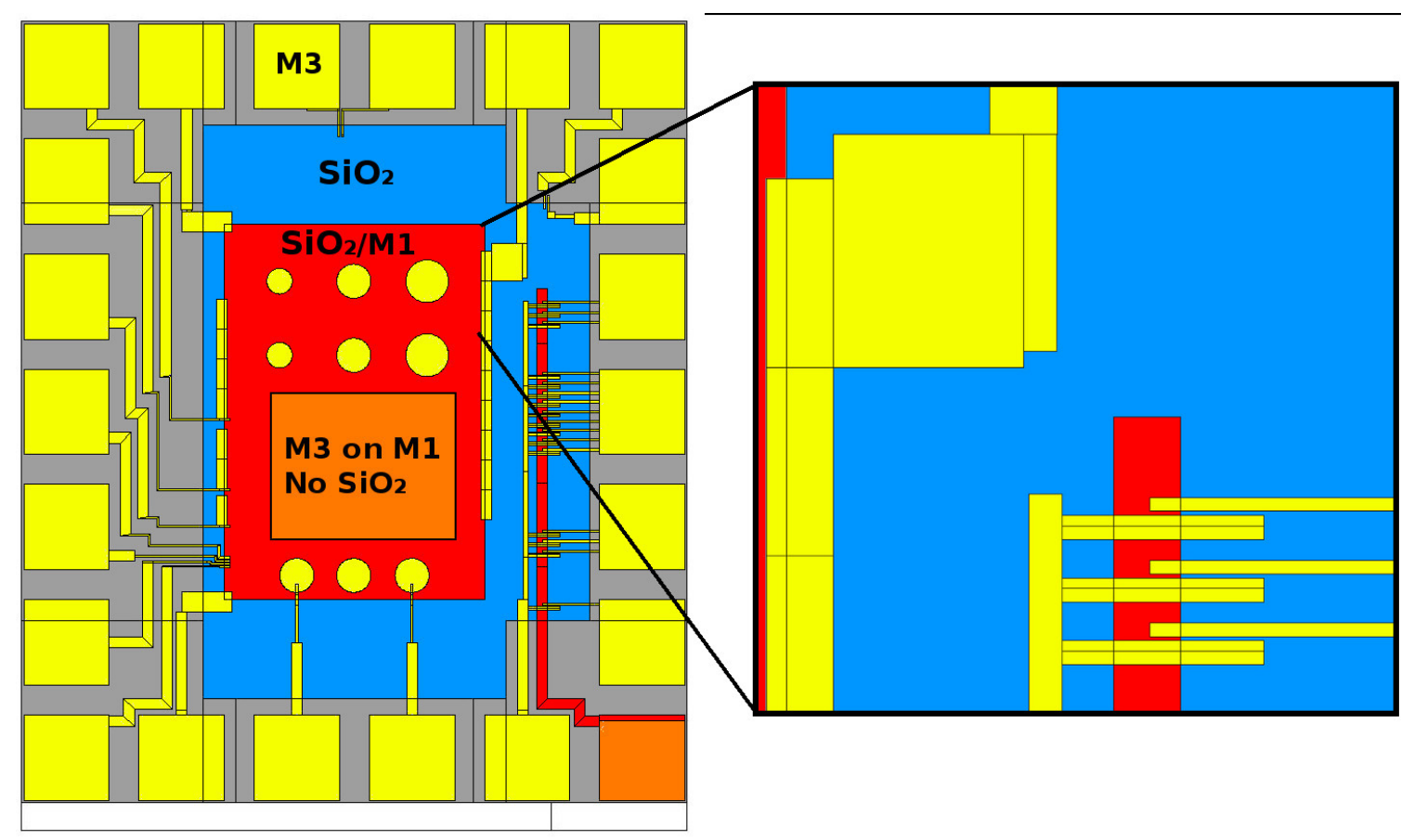

Figure 4.8: Left: CAD of M3 etch test block. Right: Close view of overlap test elements, M3 lines along the insulated M1 step test for shorting of M3 to M1 through the insulation. Thin M3 lines overlapping insulated M1 and separated by $3 \mu \mathrm{m}$ test for completeness of the M3 etch along sidewalls, which has been shown to cause device failure shown in Figure 4.11
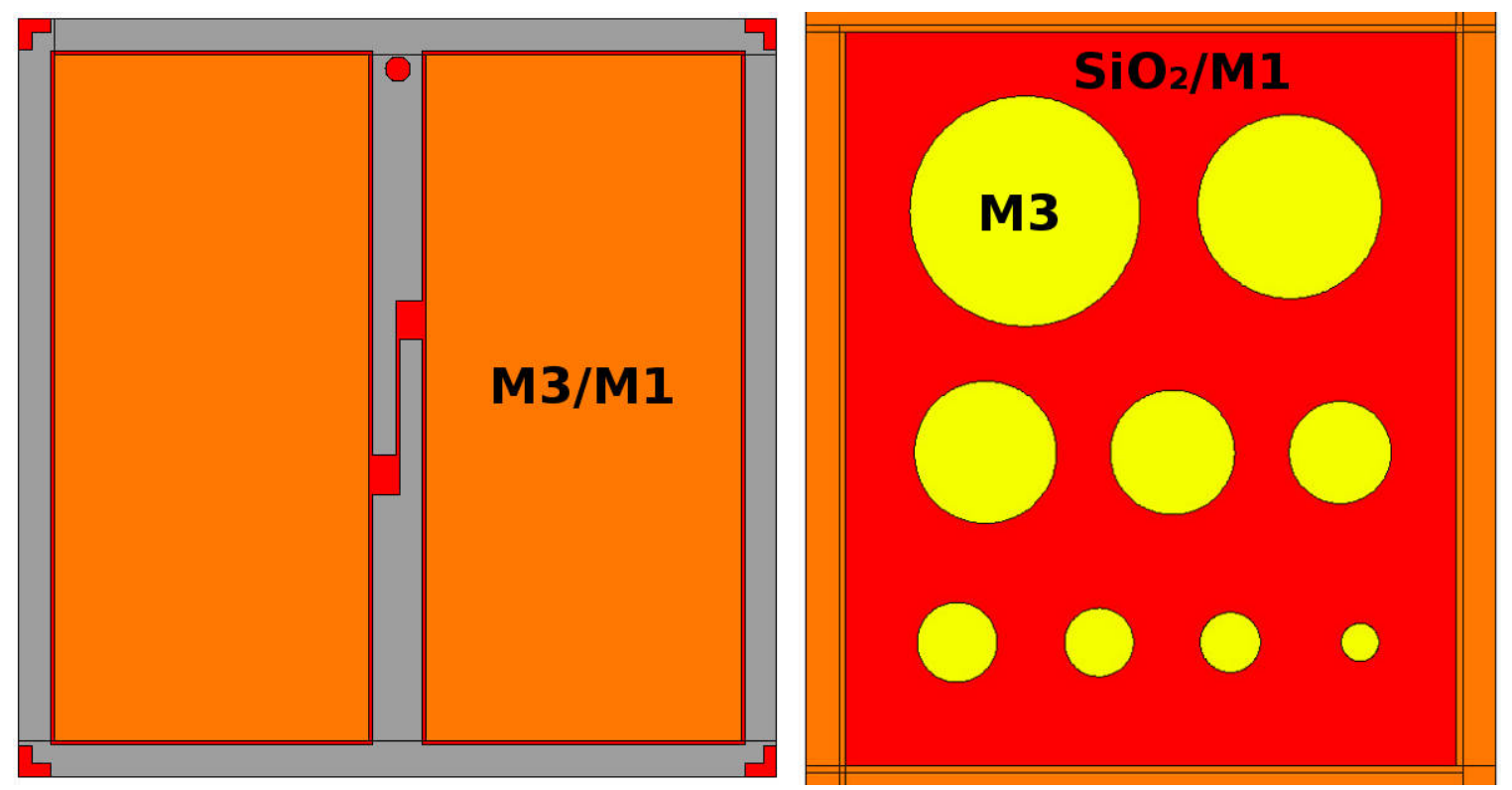

Figure 4.9: Left: $\mathrm{T}_{c}$ test block, the resistance of the thin M1 line is monitored as the block is cooled to measure the transition temperature. Right: Parallel plate capacitors to measure dielectric constant of the $\mathrm{SiO}_{2}$ film. 


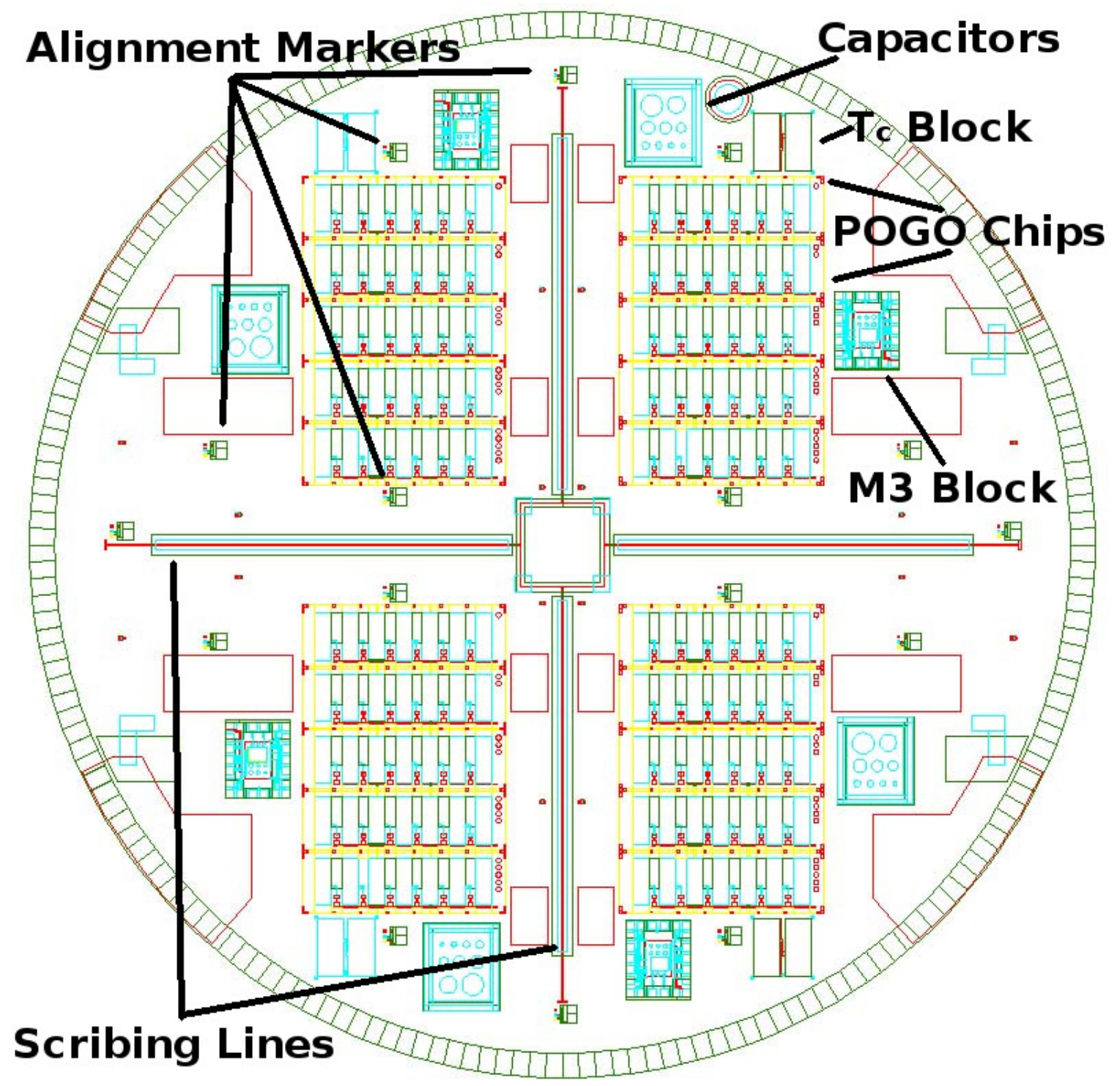

Figure 4.10: CAD view of the entire Test Mask set for use with a $50 \mathrm{~mm}$ substrate. All relevant test blocks and features are labeled. One can see how this mask set is designed to be scribed into four quarters at any point in the fabrication process.

\subsubsection{Wiring Layer and Junction Insulation Test Block}

As shown in Figure 4.1, electrical connection to the junction is made with a M3 wiring layer and is insulated from the base Nb layer through deposition of an insulating film. In many mixer designs, multiple SIS junctions are connected in series through etched islands in the trilayer material and connections through the $\mathrm{Nb}$ wiring layer. 
The wiring layer geometry defines the RF transmission lines, and often contains line widths as small as $2 \mu \mathrm{m}$. A deviation in size causes a change in line impedance, for this reason the wiring layer is defined via high resolution photoresist and anisotropic RIE. Difficulty arises in etching of the wiring layer, as it covers a wide range of topography from previous etching and deposition steps. For series junction arrays, the wiring layer between adjacent junctions must be completely removed, else a short circuit between the junctions will occur. This can become problematic for edge and step topography with the use of an anisotripic RIE, exacerbated by surface roughness and folds/fissures which may develop through the various fabrication processes. Figure 4.11 is an SEM of an ALMA Band-8 mixer chip after fabrication and testing, which clearly shows a bridge of M3 shorting the junction array along the folds of the $\mathrm{SiO}_{2}$ edge coverage. The mixer demonstrated short circuit characteristics and it wasn't until post-fabrication SEM investigation that the cause of the short was realized. It is often difficult to isolate the cause of a short circuit. Short circuits can occur from: a poor quality barrier layer in the SIS, shorting through the $\mathrm{SiO}_{2}$ on the open field around the junction; shorting through the $\mathrm{SiO}_{2}$ on the edge/perimeter of the trilayer islands; and shorting through unetched linking lines of the wiring layer as observed in the Band-8 mixer. For this reason, a test block was designed and implemented on the new test mask, shown in Figure 4.8. The purpose of this test block is to troubleshoot and determine causes of failure when short circuited junctions are observed and can be easily incorporated in any mask design.

\subsubsection{Wiring Layer Fingers}

As shown in Figure 4.8, the first feature of the test block is a series of parallel lines, separated by $3 \mu \mathrm{m}$, in the wiring layer. These lines overlap an $\mathrm{SiO}_{2}$ insulated M1 step, simulating the feature shown in Figure 4.11. If the wiring layer etch is incomplete and a fence of M3 exists, it is observed as a resistance between the large macroscopic 


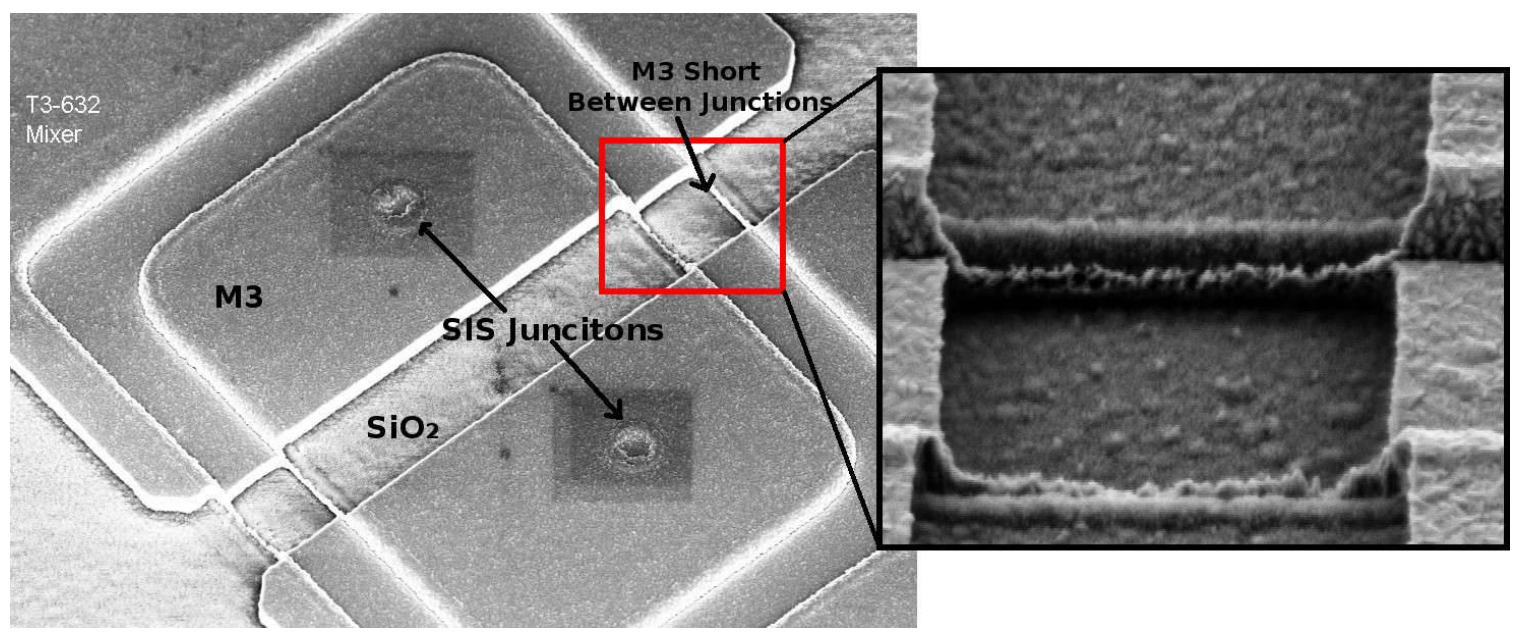

Figure 4.11: SEM micrograph of mixer which demonstrated short circuit characteristics. After further inspection, it was observed that the M3 etch was not complete, leaving $\mathrm{Nb}$ along the edge of where the $\mathrm{SiO}_{2}$ coated the $\mathrm{SiO}_{2}$ insulated $\mathrm{M} 1$ sidewall. Noting this, the test block shown in Figure 4.8 was created in order to test for M3 shorts.

pads which connect to the fingers.

\subsubsection{2 $\quad \mathrm{SiO}_{2}$ Edge and side Overlap Coverage}

As shown in Figure 4.8, a portion of the M3 test block is used to determine whether or not the junction sealing $\mathrm{SiO}_{2}$ also completely seals the edges and corners of the underlying M1 layer. A failure in the step coverage could cause an electrical short between the M3 and M1 in series with the SIS junctions. Various amounts of overlap are included (ranging from 10 to $1200 \mu \mathrm{m}$ ), as in mixer designs, line widths are on the order of microns and it may be difficult to observe shorting through the edges if the failure in step coverage is sporadic.

\subsubsection{3 $\quad \mathrm{SiO}_{2}$ Pinholes}

Pinholes in the insulation layer can manifest themselves as conduction paths from M3 to M1 in the open field, producing short circuits in parallel with the SIS junctions. The features on the test block to detect pinholes are circular electrodes defined in the 
M3 wiring layer, which sit on top of $\mathrm{SiO}_{2}$ insulated M1 layer as shown in Figure 4.9. Pinholes are detected through measurement of a resistance between the top electrodes and the base trilayer, indicating a conduction path through the $\mathrm{SiO}_{2}$. Further insight on pinhole density can also be inferred from DC resistance measurements of the parallel plate capacitors described in the following subsections that have areas ranging from 23,000 to $841,000 \mu \mathrm{m}^{2}$

\subsubsection{Transition Temperature $\left(T_{c}\right)$ Block}

Additional test elements from previous work designed to measure $T_{c}$ of the superconducting films and the dielectric constant of the junction insulation are included and shown in Figure $4.9[21,39,54]$. The transition temperature of a superconducting thin film is often indicative of film quality, decreasing with increasing impurity concentration or high levels of intrinsic stress, and correlates to the energy gap, $2 \Delta$, of a given material through the BCS relation (at $0 \mathrm{~K}$ ):

$$
2 \Delta(T) \approx 3.52 k T_{c}
$$

Noting this, measurement of $T_{c}$ is one of the fundamental characterization techniques used for optimizing deposition conditions of superconducting films, especially for higher $\mathrm{T}_{c}$ alloys such as NbTiN. A reduced $\mathrm{T}_{c}$ for a given film, while all other conditions remain constant, is often indicative of material contamination. A simple method of measuring transition temperature is through monitoring the change in resistance of a superconducting thin film as it is cooled below $T_{c}$. The test block consists of two large, 1x3 mm, pads separated by a thin, 15x465 $\mu \mathrm{m}$, Nb line. The geometries of the line are such that it is highly resistive at room temperature and the transition to the superconducting state can be easily observed through monitoring the resistance between the two macroscopic pads. 

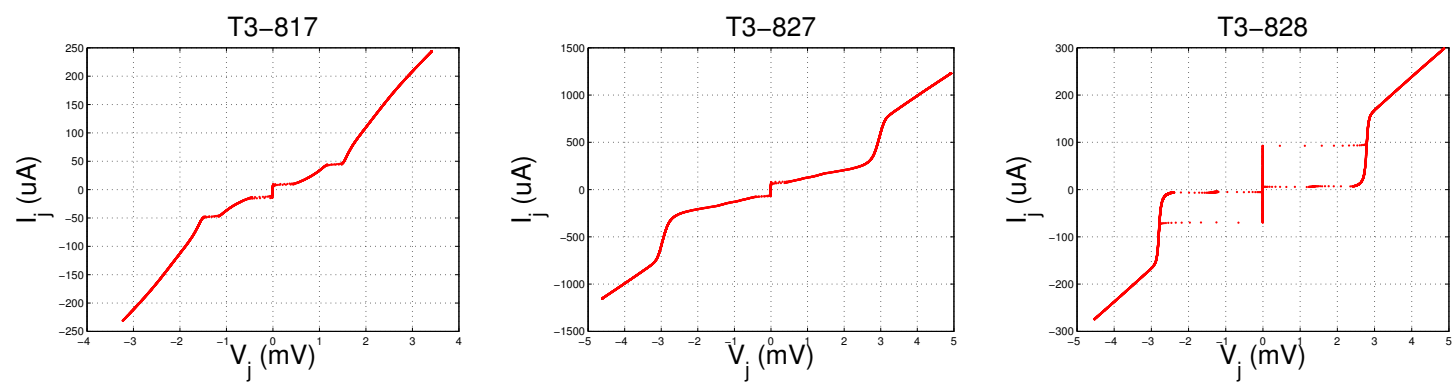

Figure 4.12: Left: Trilayer produced with tungsten contaminated target. Middle: First batch of trilayer produced after superficial cleaning of the Nb target. Right: Batch of trilayer produced after sputtering the Nb target for several hours to remove contamination. The progressive increase in junction quality after cleaning, as well as increase in $\mathrm{T}_{c}$, demonstrates the influence of target contamination on junction quality.

During a few months span, our research group noticed a decrease in junction quality and reduction in gap voltage, as shown in the IV in the far left of Figure 4.12. Through use of the $\mathrm{T}_{c}$ test blocks, we measured a reduced $\mathrm{T}_{c}$ of $8.7 \mathrm{~K}$, compared to our nominal $9.2 \mathrm{~K}$ observed in our sputtered $\mathrm{Nb}$ films. This indicated a problem with our Nb films - noting this, we vented our deposition tool and found that our $\mathrm{Nb}$ target was covered in fragments of tungsten. Pressure is monitored in our tool through the use of a naked ion gauge, which extends into the chamber for accurate readings. When one of the ion gauge filaments failed, its debris landed on our $\mathrm{Nb}$ target. After the tungsten was removed from the target, and deposition conditions were reestablished, the $\mathrm{T}_{c}$ returned to $9.2 \mathrm{~K}$. The first batch of trilayer deposited after the cleaning demonstrated slightly better quality junctions, while the subsequent batch, produced after many hours of sputtering the target to remove and residual contamination, produced high quality trilayer, as shown in Figure 4.12. This leads us to believe tungsten contamination is the culprit, and demonstrates the importance monitoring $T_{c}$. 


\subsubsection{Dielectric Measurement Block}

The final test block for this mask set is designed to measure the dielectric constant of the $\mathrm{SiO}_{2}$ film. In addition to providing electrical insulation of the SIS junctions, the $\mathrm{SiO}_{2}$ film also provides the dielectric material for the $\mathrm{RF}$ transmission and affects their impedance and propagation constant. As the material properties of thin films, produced through various deposition methods and conditions, vary from their bulk values, precise knowledge of their value is integral to proper RF design. They consist of 9 circles, with diameters ranging from 170 to $1035 \mu m$, sitting on top of the $\mathrm{SiO}_{2}$ insulated trilayer, forming parallel plate capacitors. In addition to measuring the dielectric constant of the $\mathrm{SiO}_{2}$ layer, it also provides information on pinhole density if resistance is observed through the dielectric (see Figure 4.9).

\subsection{Results from Al Quadlevel Process and New Test Mask}

After optimizing the individual steps of the $\mathrm{Al}$ quadlevel process, shown in detail with the process sheets provided in Appendix IV, several junctions of high quality were produced as shown in Figure 4.12 , a quantitative analysis of the $\mathrm{Al}$ quadlevel process and use of the newly designed test mask was performed. Al-oxide based SIS trilayer, with a modest $\mathrm{J}_{c}$ of $5 \mathrm{kA} / \mathrm{cm}^{2}$, was deposited on $330 \mu \mathrm{m}$ thick, $50 \mathrm{~mm}$ diameter DSP Si wafers with $2500 \AA$ of thermally grown $\mathrm{SiO}_{2}$, using the deposition system described in Chapter 3. The reason for Al-oxide based trilayer is that it is well researched and typically has a very repeatable $\mathrm{J}_{c}$, and our group has produced high quality trilayer with a $\mathrm{J}_{c}$ of $5.25 \mathrm{kA} / \mathrm{cm}^{2}\left(\mathrm{R}_{N} \mathrm{~A}\right.$ of $\left.34 \Omega \cdot \mu m^{2}\right)$ for the ALMA Band-6 project. For this reason, we argued the use of Al-oxide trilayer as a known high quality standard.

Using the new test mask's ability to separate the wafer in four pieces along any step of the deposition process, the wafer was quartered before the junction insulation 
step to investigate the differences between four $\mathrm{SiO}_{2}$ deposition methods available in our research laboratory. The subsequent $\mathrm{Nb} / \mathrm{Au}$ wiring layer was defined via liftoff using an NFR/AZ-5214 stack, and all four quarters were loaded into the deposition tool together on a simple substrate holder and deposited simultaneously.

There are different figures of merit with regards to junction quality in the literature. The current rise, $I_{S S}$, is defined as the value of the current rise where the nonlinearity of the IV transitions into the linear region. The gap voltage, $V_{g a p}$, is defined as the voltage at $50 \%$ of the current rise at the non-linearity, and the voltage range of which this occurs, $\Delta V$, is defined as the difference between the voltages at $90 \%$ and $10 \%$ of the current rise. The normal state resistance, $R_{N}$, is read as the resistance of the linear region after the gap voltage. Similarly, the subgap resistance, $R_{S G}=\frac{V_{S G}}{I_{S G}}$, is read as the resistance below the gap voltage, often taken at $V_{S G}=$ $2.0 \mathrm{mV}$ (though occasionally at voltages from $1.8-2.2 \mathrm{mV}$ ) in the literature - in this work, we will use $V_{S G}=2.0 \mathrm{mV}$. From these quantiles, one can calculate junction parameters such as $J_{c}$ and $R_{N} A$. The critical current density is found empirically to be:

$$
J_{C} \approx 0.7 \Delta I_{S S}
$$

and depends on an assumed strength of the coupling of the wavefunctions. Similarly, $R_{N} A$ is simply the product of $R_{N}$ and the junction area. Because $R_{N}$ can be read directly from the IV without assumptions of the coupling factor, we focus on $R_{N} A$ values instead of $J_{c}$. Our figure of merit is the ratio of the normal state junction resistance to the subgap resistance $\left(R_{N} / R_{S G}\right)$ which is commonly used in the literature where larger values indicate higher quality junctions with lower subgap leakage $[55,56]$. Figure 4.13 shows an IV curve of an SIS junction fabricated by our group at $4.2 \mathrm{~K}$ with pertinent quantities labeled. 


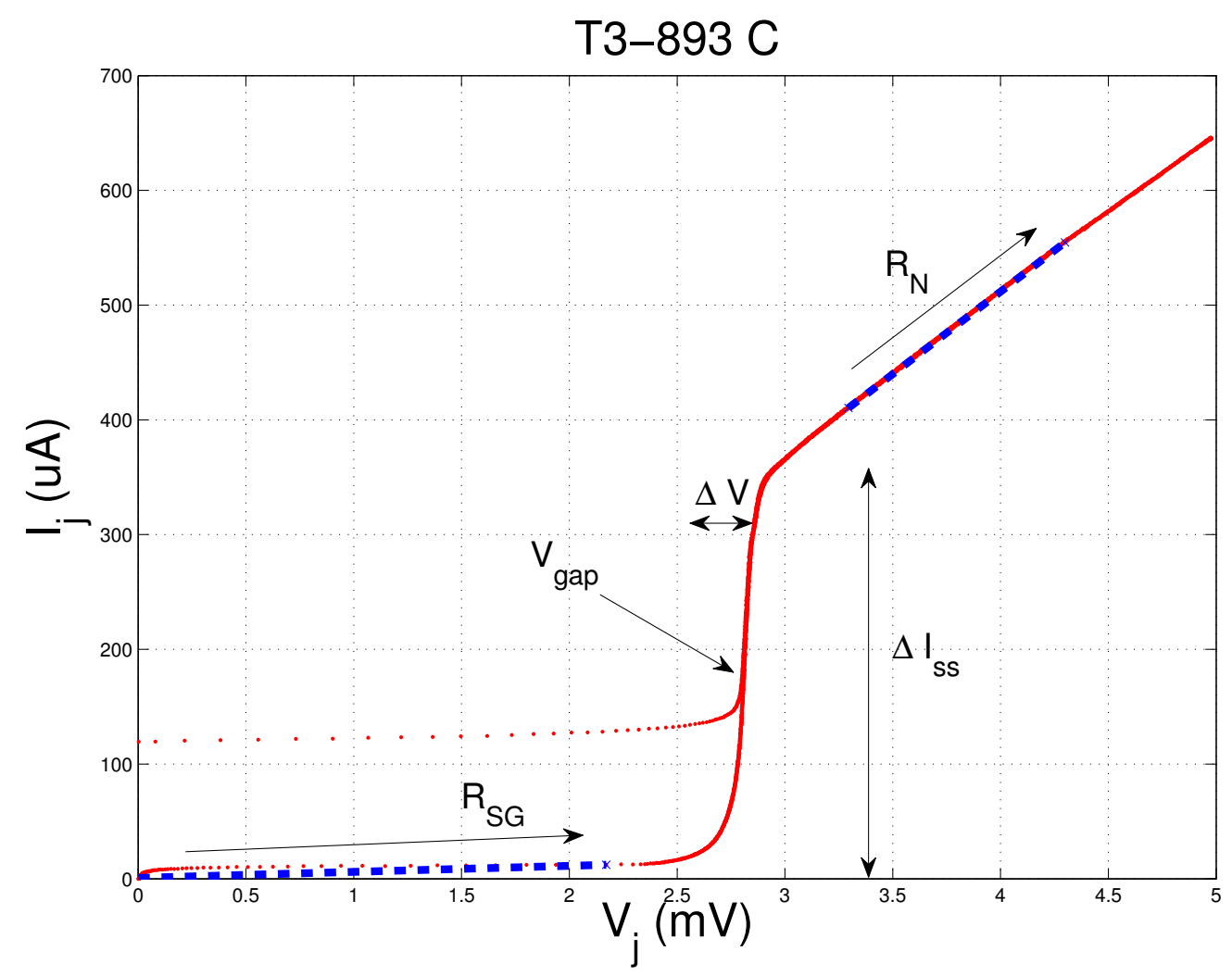

Figure 4.13: SIS curve depicting $R_{N}, R_{S G}, \Delta I_{S S}, V_{g a p}$, and $\Delta V . R_{S G}$ is taken as the average resistance at $\pm 2 \mathrm{mV}$, as indicated by the dashed line, and discussed in Section 4.4.1.

\subsubsection{Testing Setup}

The POGO chips were tested by submersion in liquid helium, using the aforementioned dip stick, and IVs were recorded through a four point measurement using a GRAPHTEC GL-900 Midi Logger and a current bias. The subgap resistance is taken as a linear fit to the IV from -2 to $2 \mathrm{mV}$, and is determined from the raw IV data as part of our group's IV plotting software (developed as part of this research), the code of which is shown in Appendix $\mathrm{V}$. The reason for taking a fit from -2 to $2 \mathrm{mV}$, instead of taking just the resistance at $+2 \mathrm{mV}$, is that we take into account the symmetry of the IV, $R_{S G}(2 m V)=R_{S G}(-2 m V)$, and any error from an offset in the zero reference in the testing setup, as shown as an XY shift in the IV is removed. The 
results are discussed below.

\subsubsection{Comparison of Different $\mathrm{SiO}_{2}$ Deposition Methods}

Four methods of liftoff-compatible $\mathrm{SiO}_{2}$ deposition are readily available in our research laboratory. Traditionally, our research group used sublimated $\mathrm{SiO}_{x}$ through use of a retrofitted Vacuum Industries model 1806 thermal evaporator. The $\mathrm{SiO}_{x}$ film successfully produced high quality junctions and mixers for projects such as ALMA Band 3, Band 6, and SORAL Supercam, but has known limitations. Evaporated $\mathrm{SiO}_{x}$ films are readily known to contain pinholes, have a dielectric constant different than that of bulk $\mathrm{SiO}_{2}$, and may vary run to run depending on how much $\mathrm{Si}$ is incorporated in the film. For mixers and SIS test elements, with short line widths of 2-10 $\mu \mathrm{m}$ and total lengths of $\sim 200-500 \mu \mathrm{m}$, the probability of overlapping a pinhole is small enough to realize functioning devices, but for larger area test elements, such as those use to measure penetration depth and dielectric constants, the use of $\mathrm{SiO}_{x}$ was found to result in short circuits. Since the properties of evaporated $\mathrm{SiO}_{x}$ differ from that of bulk $\mathrm{SiO}_{2}$, not being able to measure the dielectric constant can become problematic for realizing RF components corresponding to design values. Additionally, when a longer over-etch is used, as is the case for heatsunk Nb junction etches, we etch further into the substrate, creating a significant step for $\mathrm{SiO}_{x}$ coverage. Because thermal evaporation is a low energy line of sight deposition process, step coverage is poor compared to higher energy deposition methods such as sputtering, and may not be able to fully cover and insulate the additional step height. For these reasons, additional deposition methods are investigated as part of this dissertation.

The first method investigated is the use of a parallel plate diode sputter tool with a 6" target, described elsewhere in previous work [21]. In my previous thesis, it was demonstrated that an advantage of using diode sputtered $\mathrm{SiO}_{2}$ is that it can produce pinhole free films, and has demonstrated the ability to form both parallel plate ca- 
pacitors, and large, several mm long, superconducting microstrip transmission lines without measurable DC leakage through the dielectric. However, one consideration that should be noted is that the wafer is exposed to an Ar plasma during definition. Although the platen which holds the substrate is water cooled, the proximity to the plasma leads to localized heating of the substrate's surface, and when substrates of poor thermal conduction are used, such as fused quartz, we have observed clear transition from Thornton Zone-1 growth, characterized by small circular grain structures, to Zone-T growth characterized by long columnar growth structures. Transition to Zone $\mathrm{T}$ growth occurs when adatoms (atoms on the film's surface after arrival) have increased energy, either through reduction in operating pressure, or increased substrate temperature. Zone- $\mathrm{T}$ growth, as a result of increased substrate temperature, have been observed to fully encapsulate micron sized self-aligned structures, making proper liftoff of junction insulation impossible, as shown in Figure 4.14. Zone $\mathrm{T}$ growth is not often observed when a Si substrate is used, as is the case with test devices, however we note that some mixers, such as those used in the ALMA Band$3 / 6$ projects, are fabricated on insulating quartz substrates. To alleviate these issues when quartz substrates are used, the wafers are first heat sunk to larger Si carrier through use of Apiezon L-grease spread over the entire bottom surface through use of a hotplate. The result is better substrate cooling and easier liftoff of Zone 1 films.

With the recent arrival of a dual chamber AJA Orion 10 Gun Sputtering tool in our research lab, two additional methods of deposition, through the use of planar RF magnetron sputtering, became available. Magnetron sputtering utilizes magnets situated below the target to greatly confine the plasma in the vicinity of the sputtering target, and the planar gun configuration allows a larger target to wafer distance, as the substrate holder no longer functions as an electrode as is the case in a parallel plate diode sputter configuration. The confinement of the plasma combined with the larger target to wafer separation results in an increased plasma density above the target 

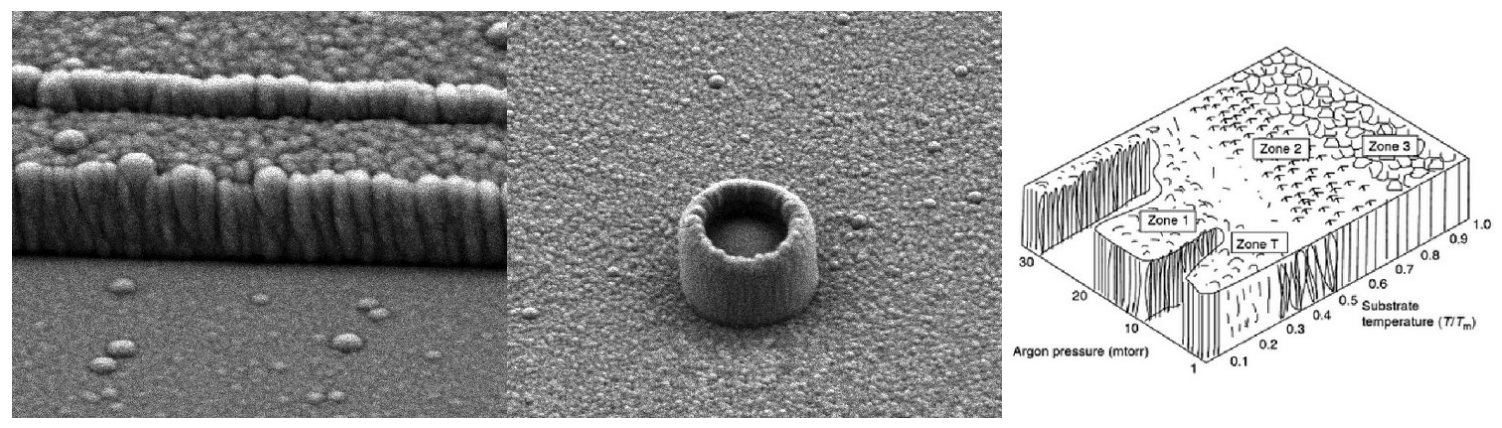

Figure 4.14: Left: Cross section of diode sputtered $\mathrm{SiO}_{2}$ demonstrating Zone $\mathrm{T}$ growth. Middle: Self aligned junction feature which failed to liftoff after deposition, large gains extending from base to the top are shown. Right: Figure of Zone 1, T, 2, and 3 growth Zones. Higher growth zones may be observed by lowering pressure, and/or increasing temperature [57].

material, away from the water cooled sample. Our AJA Orion tool is configured with both $\mathrm{SiO}_{2}$ magnetron sputter guns operating in a balanced configuration. Samples are clamped to an inconel substrate holder with Apiezon L-grease at a thermal interface material (TIM), which is suspended above the guns by a rotating holder, and actively water cooled to $15 \mathrm{C}$. The first gun accepts 2 " diameter targets and is configured in an off axis configuration. The off axis configuration is expected to provide better coverage of the junction sidewalls and trilayer steps through the $\mathrm{SiO}_{2}$ flux reaching the rotating sample at an angle. However, because in the AJA configuration, the highest deposition rate is positioned just off the perimeter of the wafer holder for excellent thickness uniformity of the deposited $\mathrm{SiO}_{2}$ film; the trade off is significantly increased target erosion. Noting the relatively small size and low off-axis deposition rate, a high applied RF power density is required and the high energy density of the plasma requires bonding of the $\mathrm{SiO}_{2}$ target to a $\mathrm{Cu}$ backing plate to avoid overheating and thermal cracking, which reduces the effective target thickness to $50 \%$ of its nominal value. Therefore, a drawback of the 2" gun, compared to the on-axis 3" gun, is the decreased target thickness, and increased target erosion that requires constant monitoring and frequent replacement of the $\mathrm{SiO}_{2}$ target. An additional limitation worth noting is that the 2 " gun design requires an $\mathrm{Al}$ chimney to be situated above 
the target in order to accommodate the deposition shutter. The chimney collects $\mathrm{SiO}_{x}$ material, which eventually delaminates and falls onto the target as shown in Figure 4.19. Constant monitoring, disassembly, and sandblasting of the 2" gun is required to avoid film contamination due to buildup of debris on the chimney.

The second $\mathrm{SiO}_{2}$ gun available on the AJA Orion tool accepts 3" targets and is situated directly below the sample in a line of sight configuration. The increased deposition rate from a line of sight configuration allows lower power densities (21 $\mathrm{W} / \mathrm{cm}^{2}$ compared to $95 \mathrm{~W} / \mathrm{cm}^{2}$ ), and the use of a full thickness target without a $\mathrm{Cu}$ backing plate. Additionally, the shutter design of the 3" gun allows configuration without the use of a chimney, removing the worry of contamination from material build up.

All four deposition methods were used as junction insulation on a single $50 \mathrm{~mm}$ trilayer wafer, scribed into four pieces before junction insulation deposition utilizing the new mask set.

\subsubsection{Ellipsometry Analysis}

Before deposition onto the four quarters, additional test samples of all four films were deposited on $300 \mu m$ thick Si for ellipsometry analysis. A baseline commercial sample of $300 \mathrm{~nm}$ of thermally grown $\mathrm{SiO}_{2}$ on a $450 \mu \mathrm{m}$ Si substrate, provided by Virgina Semiconductor, is included for comparison. The evaporated $\mathrm{SiO}_{x}$ films were deposited with an applied power of $175-185 \mathrm{~W}$ to achieve a deposition rate of $\sim 240 \AA / \mathrm{min}$ at an original base pressure of 5e-7 Torr. Diode sputtered films were deposited with an applied RF power of $90 \mathrm{~W}$ at an operation pressure of $10.0 \mathrm{mT}$ Ar, producing a typical deposition rate of 40-50 $\AA /$ min. Magnetron sputtered films were deposited at $3.0 \mathrm{mT}$ of $\mathrm{Ar}$, and applied power of $300 \mathrm{~W}$ and $150 \mathrm{~W}$ for the 2" and 3" targets respectively, producing typical depositions rates between $10-20 \AA / \mathrm{min}$. We note the off axis 2" gun is run at a high power density $\left(95 \mathrm{~W} / \mathrm{in}^{2}\right)$ compared to the on axis 3" 
gun $\left(21 \mathrm{~W} / \mathrm{in}^{2}\right)$ to produce similar deposition rates between the two guns.

Analysis was performed using a Jobin Yvon UVISEL tabletop spectroscopic ellipsometer, scanning from 230.8 to $770.8 \mathrm{~nm}$ in $10 \mathrm{~nm}$ increments, and optical constants were calculated using their included Delta Psi software package. The complex indices of refraction, $\tilde{n}=n+i \kappa$ (discussed in greater detail in Chapter 5 ), of all four deposited films as well as the thermally grown baseline, are shown in Figure 4.15a and $4.15 \mathrm{~b}$. The extinction coefficient, $\kappa$, describes the attenuation of electromagnetic radiation through scattering or absorption processes. Noting the indices of refraction, the attenuation constant of a microstrip line is:

$$
\alpha_{d}=\frac{2 \pi f \epsilon\left(\epsilon_{e}-1\right) \tan (\delta)}{c \pi \epsilon_{0} \sqrt{\epsilon_{e}}(\epsilon-1)}
$$

where $\epsilon=\epsilon_{1}+i \epsilon_{2}=\tilde{n}^{2}$ is the dielectric constant of the insulating film, $\epsilon_{e}$ is the effective dielectric constant due the microstrip geometry, and $\tan (\delta)$ is the loss tangent where $\delta=\epsilon_{2} / \epsilon_{1}[58]$. Noting $\alpha_{d} \propto f \cdot \tan (\delta) \approx f \cdot \delta$ for small values of $\delta$, this predicts $\mathrm{RF}$ loss in transmission lines approximately proportional to the non-zero extinction coefficients and should scale with frequency. We note that the recorded data is taken from 230-700 $\mathrm{nm}$ and the extinction coefficient increases sharply as wavelengths approach the band edge of $\mathrm{SiO}_{2}$ at $\sim 140 \mathrm{~nm}$ before leveling off at larger wavelengths. For THz frequencies, wavelengths are 3 orders of magnitude larger than optical wavelength and the results from Figure $4.15 \mathrm{~b}$ are not directly applicable. However, the optical measurements of the index of refraction provide insight to the relative differences between the various deposition methods. With this in mind, we note evaporated $\mathrm{SiO}_{2}$ demonstrated the largest deviation from the thermally grown baseline, followed by the diode sputtered $\mathrm{SiO}_{2}$. The large refractive index and extinction coefficient of evaporated $\mathrm{SiO}_{x}$ indicates it will have the largest difference in dielectric constant and is the lossiest dielectric of the group. The magnetron sputtered $\mathrm{SiO}_{2}$ provides the closest match to the thermal $\mathrm{SiO}_{2}$ baseline, with the 3 " gun providing a near perfect 
match.
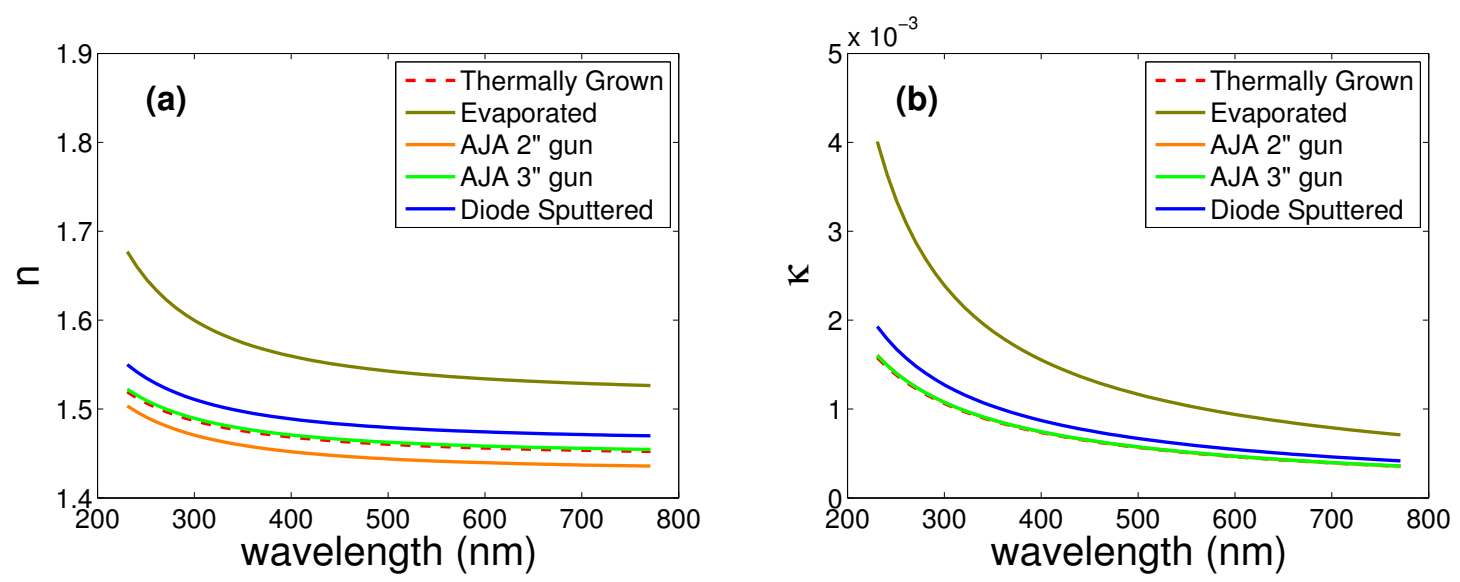

Figure 4.15: Complex index of refraction, $\vec{n}=n+i k$, for available $\mathrm{SiO}_{2}$ films.

\subsubsection{Junction Liftoff Profile}

After the M2 Nb/Au junction etch, the junction insulation is defined through the self aligned NFR/polyimide/Al stack (shown in Figure 4.6), that sits atop the RIE defined M2 Nb/Au top electrode. After the junction RIE, the sample is placed into the junction insulation deposition system and the insulating film is deposited. To complete the liftoff process and expose the top electrode, the wafer is soaked in a 1:1 mixture of N-Methyl-2-pyrrolidone (NMP) and propylene glycol (p-glycol) heated to $120 \mathrm{C}$ to dissolve the polyimide for 2 or more hours, and it is swabbed several times in a bath of room temperature ethylene glycol (e-glycol) to help break up the polyimide features and facilitate the liftoff process.

Figure 4.16 shows SEM micrographs of both of the junction insulation liftoff profile around a $1 \mu \mathrm{m}$ SIS junction and insulation coverage at the edge of the trilayer features. The evaporated film is visibly the smoothest and demonstrates complete coverage around the edge of the junction. Thermal evaporation is a low energy line of sight deposition process, where the adatom mobility upon arrival is low. For liftoff processes, this can be advantageous, as the deposited film will not fully coat the relatively tall sidewalls of the self-aligned liftoff features, facilitating the ability of 
the NMP + p-glycol mixture to dissolve the polyimide and remove the self-aligned pattern, requiring minimal swabbing. The thermal evaporator, unlike the rest of the deposition systems, contains a 45 degree ion mill, which is used to clean the wafer in situ before deposition. Removal of contamination along the junction edge and substrate surface is likely to play a role in adhesion of the $\mathrm{SiO}_{x}$, resulting in better insulation around the junction.

The diode sputtered and magnetron films demonstrate Zone-1 growth and look very similar to each other, but have a visible larger grain structures and a more physically jagged resulting liftoff profile than the evaporated films. We note that sputtering is a much higher energy deposition process and the adatoms have energies about 2 orders of magnitude greater than that of thermal evaporation [59]. For the AJA off axis magnetron configuration and our diode sputter configuration (using a $150 \mathrm{~mm} \mathrm{SiO}_{2}$ target with a target to wafer separation of $125 \mathrm{~mm}$ ), significant flux arrives at the wafer at an angle; the process is much less line of sight compared to thermal evaporation. As a result of the increased adatom mobility, and non-line-ofsight deposition for the case of our off axis and diode sputter configurations, increased sidewall coverage is expected for sputter deposited films. Figure 4.17 shows an SEM of a cross section of a junction with evaporated and diode sputtered $\mathrm{SiO}_{2}$, showing the the difference in the physical sealing around the edge of the junction. The evaporated film, owing to its low energy line of sight deposition, tapers as it reaches the edge of the junction, while the higher energy sputtered film shows a slight buildup up past the junction edge.

During the liftoff process, the amount of sidewall coverage, particularly if the film coats the side of the self-aligned features, is a critical difference, as the sputtered film can potentially fail if the $\mathrm{SiO}_{2}$ physically breaks from poor adhesion or physical swabbing of the surface as shown in Figure 4.6. With this in mind, sections of the the protective junction insulation ring have periodically been shown to break off 
during the liftoff process for sputtered films, potentially due to large grain structures extending from the side wall of the $\mathrm{M} 2 \mathrm{Nb}$ junction up the side of the polyimide stack, breaking free while swabbing the sample during liftoff as shown in the right of Figure 4.17. While it is not clear if such partial breaks will lead to electrically shorting paths, we keep this in mind as we cover the results from from the 4 different junction insulation processes.

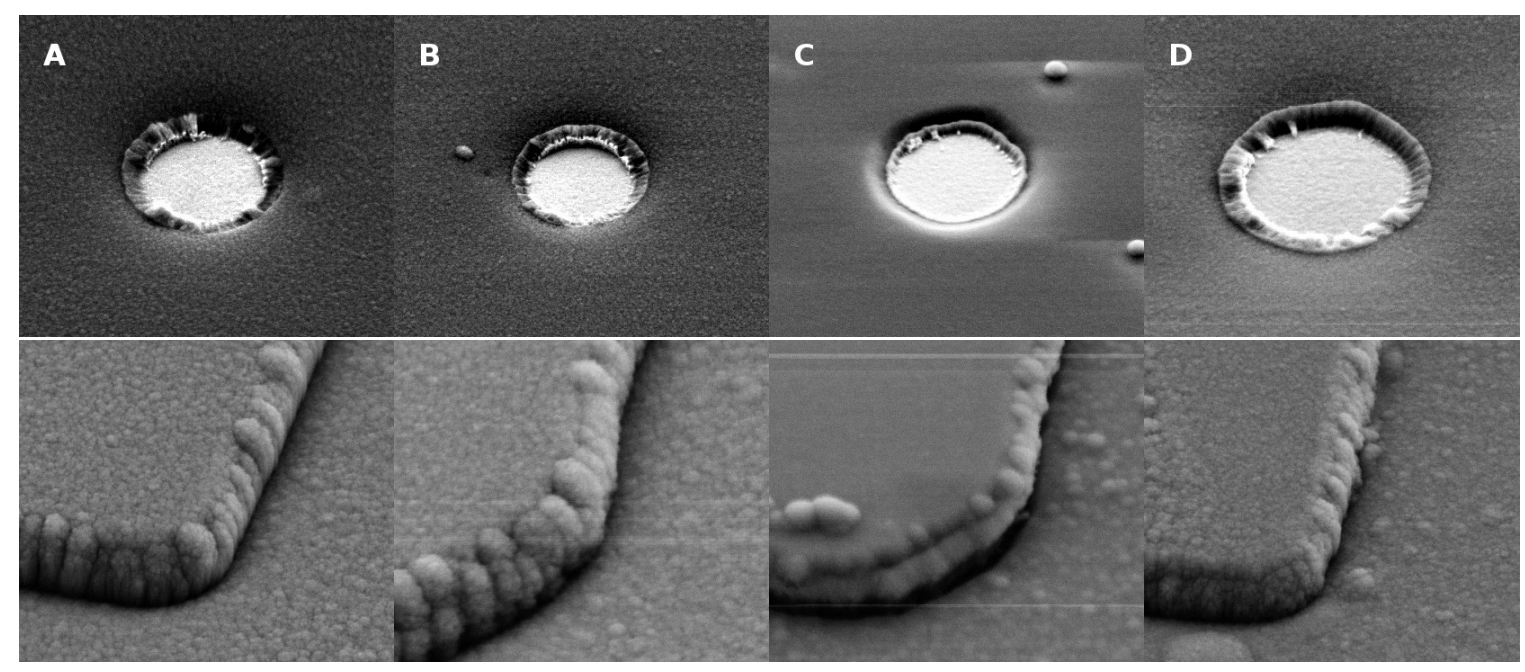

Figure 4.16: Liftoff profile of insulation film around a $1 \mu m$ junction (top) and edge of trilayer features (bottom). From left to right the deposition systems used are: A - diode sputtered, B - 2" magnetron sputtered, C - thermal evaporated, and D - 3" magnetron sputtered.

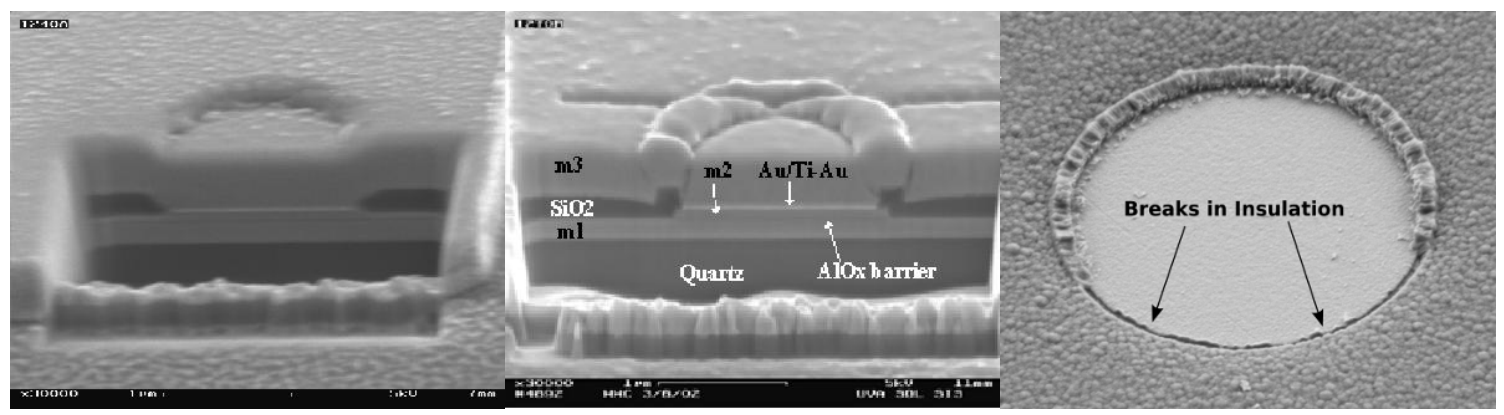

Figure 4.17: Cross section of thermal evaporated $\mathrm{SiO}_{x}$ (left) and diode sputtered $\mathrm{SiO}_{2}$ (middle). Right: Diode sputtered $\mathrm{SiO}_{2}$ after liftoff, demonstrating the occasional occurrence of breaks in the ring of the $\mathrm{SiO}_{2}$ around the junction perimeter after liftoff. 


\subsubsection{IV Results and Junction Quality}

Junctions IV characteristics were recorded at $4.2 \mathrm{~K}$ through submersion in a liquid helium bath. The device yield (percentage of tested POGO elements with SIS characteristics), $R_{S G} / R_{N}$ and its mean, max, and standard deviation, $\sigma$, are tabulated in Table 4.1 for all four sections of the wafer. Additionally, the 'no junction' test elements were tested for short circuits at $4.2 \mathrm{~K}$, and are also tabulated in Table 4.1. Each quarter of the wafer contains four $1.7 \mu m$ nominal sized $R_{N} A$ calibration junctions spread across the entire wafer, and a total of 15/16 produced SIS characteristics. The variance of $R_{N} A$ across the 15/16 $1.7 \mu \mathrm{m}$ nominal single junction was calculated to be 9.5\% and the resulting IV's are shown overlaid in Figure 4.18, visually demonstrating the consistency across a $50 \mathrm{~mm}$ substrate. The spread in $R_{N} A$ across the wafer is believed to be predominantly due to a spread in junction size across the wafer, which corresponds to a variance of $\pm 0.08 \mu \mathrm{m}$ in junction diameter for our $1.7 \mu \mathrm{m}$ nominal diameter junctions. Sources of spread in junction diameter can come from spread in size across the photolithographic mask (specified within $\pm 0.05 \mu \mathrm{m}$ tolerance by the manufacture), uniformity of the UV power density of the contact aligner (typically $\pm 1-3 \%$ ), gradients across the hotplates used for cross-linking the NFR resist, uniformity in the $\mathrm{Al}$ wet etch, and/or uniformity of the $\mathrm{Nb} / \mathrm{Au} \mathrm{RIE}$ etches. We note the pentalevel process used for the ALMA Band-8 mixers in Chapter 3, produced a variance in $R_{N} A$ of $7 \%$ for $1.0 \mu \mathrm{m}$ junctions, corresponding to a $\pm 0.04 \mu \mathrm{m}$ variance in junction diameter. Noting that the same mask company, developer, hotplates, photoresist, RIE equipment, and contact aligners were used in both this work and the pentalevel Band-8 fabrication, it is argued that the increased variance is likely due to the use of an $\mathrm{Al}$ wet etch. We note that a trade-off exists between the significantly reduced fabrication time of the quadlevel $\mathrm{Al}$ process and slight increase in the variance of junction size, yet may have advantages in certain applications discussed in the conclusions. 


\begin{tabular}{|c|c|c|c|c|c|c|c|c|}
\hline De potion Method & Mean $\frac{R_{S G}}{R_{N}}$ & $\operatorname{Max} \frac{R_{S G}}{R_{N}}$ & $\sigma \frac{R_{S G}}{R_{N}}$ & Yield & P1* & P2 & P3 & P4 \\
\hline \hline Diode Sputter & 16.2 & 27.1 & 6.4 & $86 \%$ & $\mathrm{O}$ & $\mathrm{O}$ & $\mathrm{S}$ & $\mathrm{S}$ \\
\hline 2" Magnetron & 12.9 & 19.9 & 4.0 & $90 \%$ & $\mathrm{O}$ & $\mathrm{O}$ & $\mathrm{O}$ & $\mathrm{O}$ \\
\hline Thermal Evap & 21.6 & 29.9 & 4.4 & $90 \%$ & $\mathrm{O}$ & $\mathrm{O}$ & $\mathrm{S}$ & $\mathrm{S}$ \\
\hline 3" Magnetron & 15.0 & 18.6 & 2.5 & $81 \%$ & $\mathrm{O}$ & $\mathrm{O}$ & $\mathrm{O}$ & $\mathrm{S}$ \\
\hline 2" Magnetron** & 21.3 & 27.3 & 4.9 & $67 \%$ & $\mathrm{O}$ & $\mathrm{O}$ & $\mathrm{O}$ & $\mathrm{O}$ \\
\hline
\end{tabular}

Table 4.1: Tabulated test results for various $\mathrm{SiO}_{2}$ deposition methods. *The no junction test elements are tested for "O" open circuit characteristics or "S" short circuit characteristics. P1-2 correspond to the $12 \times 45 \mu \mathrm{m}$ overlaps, P3 to the 100 x $100 \mu \mathrm{m}$ square, and P4 to the largest $125 \times 160 \mu \mathrm{m}$ overlaps. **After noting the debris formation on the 2 " $\mathrm{SiO}_{2}$ target, an additional test quarter from a separate piece of Al-oxide based trilayer was fabricated as discussed.

Noting the results of our quartered wafer, thermal evaporated $\mathrm{SiO}_{x}$ demonstrated the best results in terms of both mean and $\max R_{S G} / R_{n}$. The result was at first unexpected, as evaporated $\mathrm{SiO}_{x}$ films are known to contain pinholes and vary in stoichiometry from bulk $\mathrm{SiO}_{2}$ as confirmed by our ellipsometer measurements. We explain this result by noting none of the 12 x $45 \mu m$ no-junction test elements demonstrated any signs of conduction - for small overlaps the probability of landing on a pinhole is small. If leakage current is not observed through the open field or trilayer edge coverage, we conclude excess leakage current is manifested around the junction perimeter or through the tunnel barrier itself. However, the same trilayer wafer was used for all four samples, as such, it is argued the main difference in leakage current is due to leakage through the junction perimeter. Leakage through the perimeter can likely manifest itself in two ways: (a) through breaks in insulation around the perimeter, and/or (b) through damage of the barrier and/or $\mathrm{Nb}$ at the perimeter of the junction during the junction insulation deposition process. With this in mind, the SEM micrographs in Figure 4.16 and Figure 4.17 argue the evaporated films seal the junction in such a way to minimize the probability that sections of the protective ring can be pulled out during junction liftoff, particularly when physical swabbing is required. We also note that there is no plasma present in the evaporation process; 
plasma in the vicinity of the junction can potentially play a role in perimeter damage. The results from the diode sputtered films produced the second best results in terms of both mean and $\max R_{S G} / R_{n}$. However, the variance is much larger, and noting liftoff of diode sputtered films is often difficult due to plasma hardening of the polyimide, and the largest amount of physical swabbing of the wafer during liftoff is required, compared to both the evaporated and magnetron sputtered samples, which may partially physically disturb/break the insulation around the SIS junctions. Less physical swabbing was required during liftoff for both the 3" and 2" magnetron guns, believed to be a result of the large, $25 \mathrm{~cm}$, wafer to target separation, and plasma confinement above the target of the magnetron configuration; with no plasma in the vicinity of the sample, the possibility of plasma hardening of the polyimide is reduced compared to the non-magnetron diode sputter deposition.

The 3" target, which has a near line of sight deposition, produced the most consistent, albeit close to poorest results with the lowest maximum value of $R_{S G} / R_{N}$. The relatively low power density of the on axis deposition may play a role in how the insulation seals around the perimeter of the junction. Further tests using the 3" gun at higher power densities can potentially provide further insight to how power density may play a role for sputtered $\mathrm{SiO}_{2}$ junction insulation.

The 2" magnetron results are the most unexpected. The off axis deposition was thought to have the best junction edge coverage and a high density pinhole free film was expected as a result of the low $3.0 \mathrm{mT}$ operating pressure and large $95 \mathrm{~W} / \mathrm{in}^{2}$ power density (compared to the 3" gun's power density of $21 \mathrm{~W} / \mathrm{in}^{2}$ ) . However, on average, the largest amount of subgap leakage current was observed. As argued above, it was thought the subgap leakage is solely a result of sealing around the edge of junctions as no conduction was observed in the no-junction test elements up to 125 x $160 \mu m$ overlaps. In terms of quality, the $2 "$ magnetron gun produced the only film free of observable pinholes, while the evaporated film produced the junctions with the 


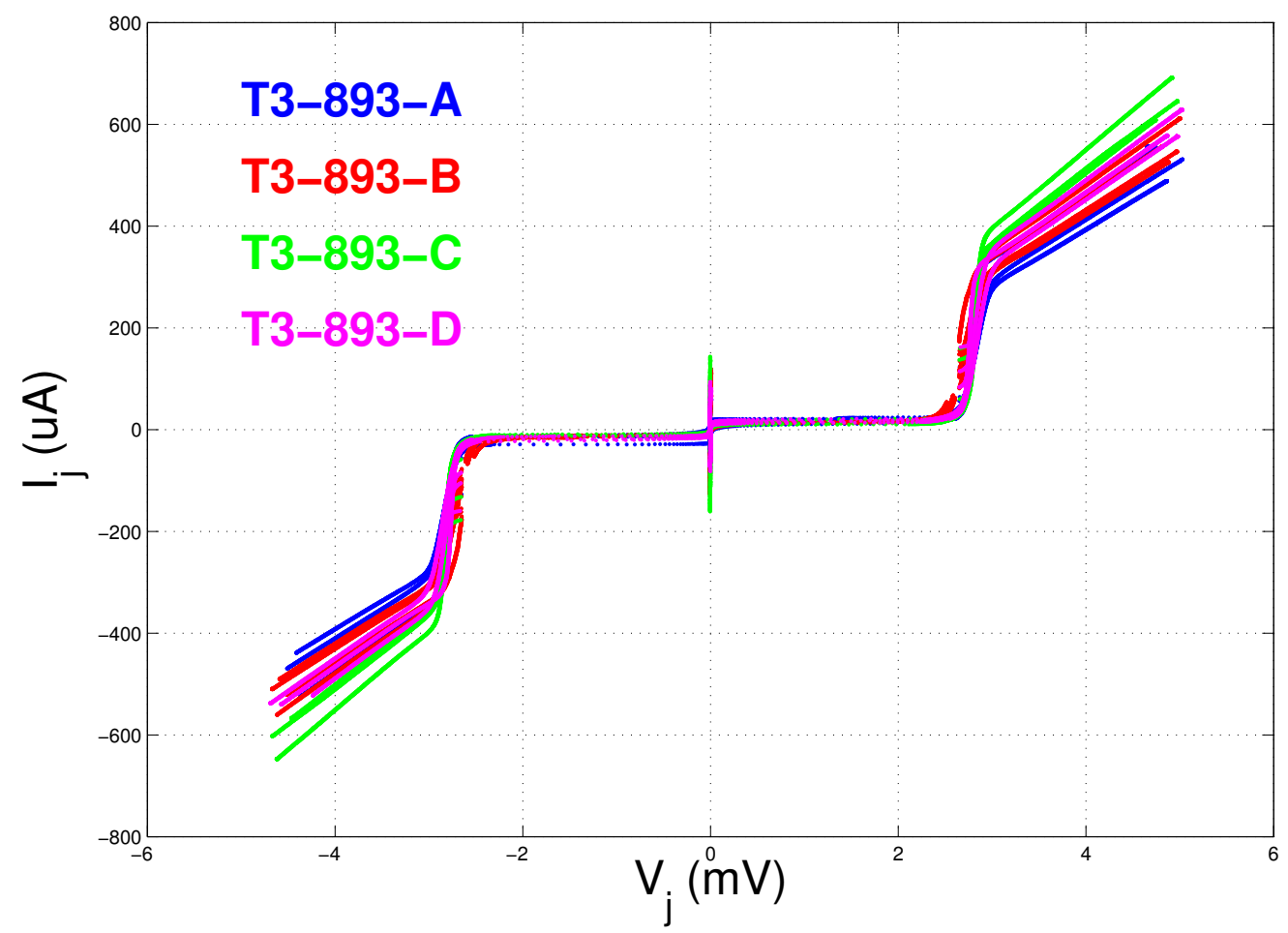

Figure 4.18: All $1.7 \mu m$ junctions plotted on the same figure. A standard deviation of $9.5 \%$ in $R_{N} A$ was calculated. Sample A, B, C, and D correspond to diode sputtered, 2 " magnetron, thermal evaporated, and 3" magnetron films respectively.

lowest subgap leakage.

We note that we have produced higher quality junctions using the 2 " $\mathrm{SiO}_{2}$ gun as we were developing the $\mathrm{Al}$ quadlevel process. However, these tests occurred before this more comprehensive study of the various junction insulations, and the 2 " target was fairly new. With this disparity in mind and owing to the increased target erosion of the off axis configuration, we first thought that the age of the target potentially played a role. When the magnetron deposition tool was vented for inspection, we discovered the 2" target was covered in dark debris, which accumulated through flaking of material off the chimney walls as shown in Figure 4.19. The debris may vary the stoichiometry of the films as well as the sputtering mechanics. Noting this, we tested POGOs from a similar Al-oxide based trilayer wafer (same $R_{N} A$ value) that 


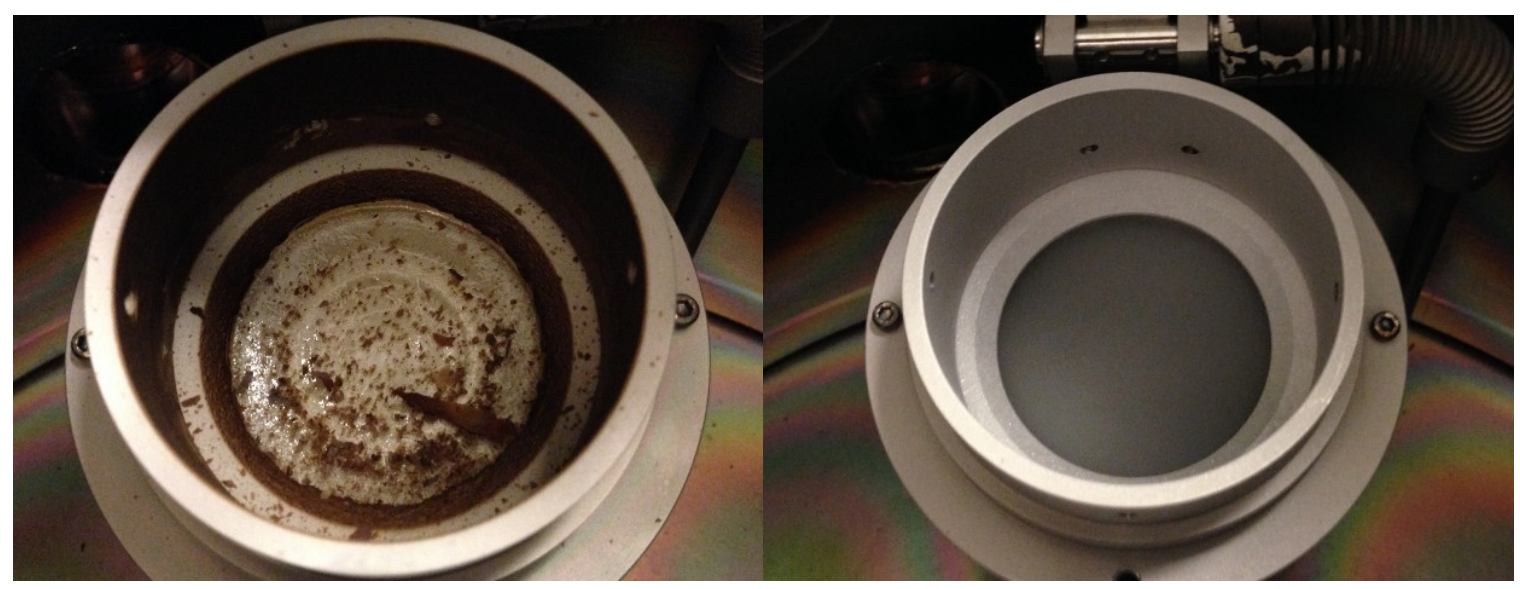

Figure 4.19: Debris cover 'dirty' $\mathrm{SiO}_{2}$ target, left, compared to newly installed 'clean' $\mathrm{SiO}_{2}$ target, right.

went through the same processing, but received the junction insulation from a clean $\mathrm{SiO}_{2}$ target. The results are tabulated in the last row of Table 4.1.

The results from the clean debris free 2" magnetron gun in terms of junction quality are comparable to the thermal evaporated $\mathrm{SiO}_{x}$ films and the contrast with the previous 2" gun results is striking. There is a modest reduction in yield, but we note, there are only 21 testable elements on each quarter section of the wafers patterned with the new test mask. A loss of one test element reduces yield by $\sim 5 \%$, and a loss of one POGO chip reduces yield by up to $\sim 25 \%$. All the failed junctions on this particular quarter were open circuit, indicating a processing error, either in junction liftoff or the wiring layer definition, and not from short circuiting through a lossy or poor quality insulation layer. The 2" magnetron gun has an additional distinct advantage as it again did not demonstrate any pinholes with overlaps of up to $125 \times 160 \mu \mathrm{m}$.

If the increase of leakage current is manifested through conduction around the perimeter of the junction, we expect to observe a reduced $R_{S G} / R_{N}$ ratio from smaller diameter junctions, where the circumference to area ratio is greatest. $R_{S G} / R_{N}$ vs junction size for the 2" magnetron samples is plotted in Figure 4.20. The junction insulated with the debris covered target demonstrate a positive correlation of $R_{S G} / R_{N}$ 

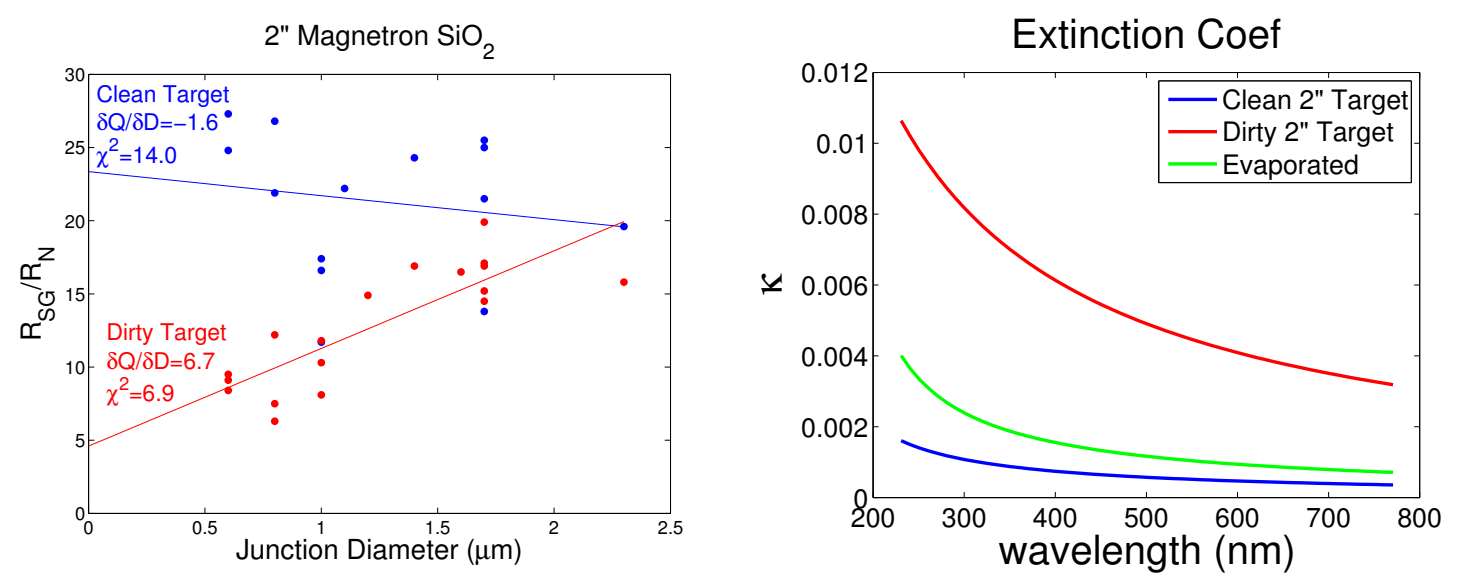

Figure 4.20: Left: $R_{S G} / R_{N}$ vs junction diameter. I note the correlation roughly as $\delta Q / \delta D$, which is the slope of a linear fit to $R_{S G} / R_{N}$, Q , and junction diameter, D. $\chi^{2}$ is the standard chi-squared goodness of fit. Right: Extinction Coefficient for clean 2 " magnetron target, dirty debris cover 2" target, and evaporated $\mathrm{SiO}_{x}$.

to junction size, approaching values close to the debris free samples for large area junctions where conduction through the junction area dominates. This evidence supports our hypothesis that conduction through the edge of a junction plays a role in the increase of subgap leakage. For further analysis, we recorded the optical constants of a $\mathrm{SiO}_{2}$ film deposited from the debris covered 2" magnetron gun to compare to the initial results shown in Figure 4.15. The results are shown in Figure 4.20, the debris covered film has the highest extinction coefficient, even more so than our evaporated films. From these results, we conclude the film deposited from the debris covered target is intrinsically different from a clean target. The extinction coefficient describes the attenuation of electromagnetic radiation through scattering or absorption processes. Both processes indicate the presence of defects; such defects can potentially introduce states in the bandgap or affect the quality of the $\mathrm{Nb}$ around the perimeter of the junction, leading to increased subgap leakage. 


\subsection{Conclusions and Future Work}

Upon the start of my research, over a year was spent fabricating SIS test junctions in order to troubleshoot issues that arose as a result of receiving an improperly configured ICP unit as mentioned in Chapter 3. As our research on AlN barriers was greatly delayed during this time, the need for a more rapid junction definition process was made apparent. In addition to a more rapid junction definition process, we often wished to investigate the variation of single particular processing step, and fabricating two wafers side-by-side was both time consuming and introduced ambiguity. Alleviating those concerns, a new Test Mask set was developed, allowing a single $50 \mathrm{~mm}$ sample to be scribed into four separate samples at any step in fabrication process. The mask set also contains test blocks to investigate and isolate different failure mechanisms observed over the years such as: shorting between M3 lines through unetched areas along sidewalls, shorting from M3 to M1 along M1 edge insulation, shorting from M3 to M1 through pinholes in the open field, and shorting through the perimeter of SIS junctions.

The highest quality junctions in this work produced from the Al quadlevel process demonstrated $R_{S G} / R_{N}$ values approaching 30 . We note that testing took place by submersion in liquid helium at $\sim 4.2 \mathrm{~K}$ - since the temperature is not absolute zero, some level of subgap leakage is expected due to the electron energy distribution. This piqued my interest for finding a fundamental limit of $R_{S G} / R_{N}$ for junctions tested by submersion in liquid helium. Using Equations (2.2-2.3) and assuming a Fermi-Dirac distribution for the quasi-particles, I generated a synthetic SIS IV at $4.2 \mathrm{~K}$ in order to calculate the ideal $R_{S G} / R_{N}$ solely due to quasi-particle excitation. The result was $R_{S G} / R_{N} \approx 56$ at $4.2 \mathrm{~K}$; Figure 4.21 plots the theoretical subgap leakage (at $4.2 \mathrm{~K}$ ) overlaid on the IV characteristics of the highest quality SIS junction tested as part of the work in this chapter. More on the temperature dependence of $R_{S G} / R_{N}$ and its implications is discussed in Chapter 7. 


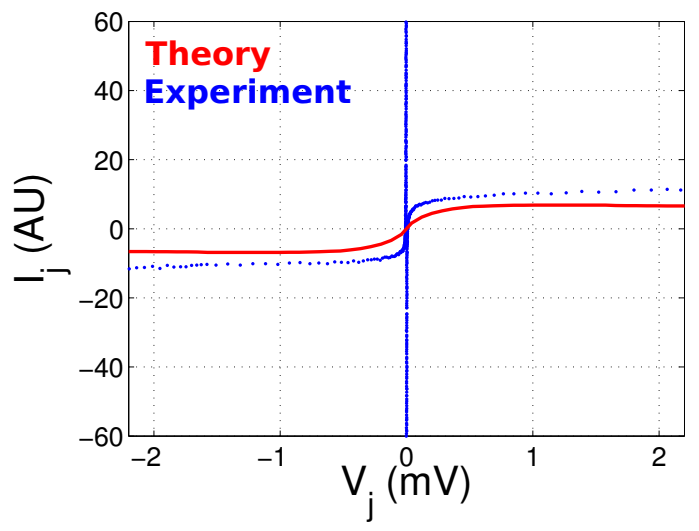

Figure 4.21: Subgap leakage predicted by BCS theory $\left(R_{S G} / R_{N}=56\right)$ overlaid on the subgap leakage of one of the highest quality junctions $\left(R_{S G} / R_{N}=27\right)$ from this Chapter.

The new Al Quadlevel process demonstrated the ability to realized high quality junctions down to $0.6 \mu \mathrm{m}$ in diameter, with a variance in junction resistance across a $50 \mathrm{~mm}$ wafer of $9.5 \%$ for $1.7 \mu \mathrm{m}$ diameter junctions. For both thermally evaporated and 2" magnetron sputtered junction insulation, the average $R_{S G} / R_{N}$ values were in excess of 20 with the maximum values within a factor of $\sim 2$ of the theoretical limit due to quasi-particle excitation at $4.2 \mathrm{~K}$. From the use of the New Test Mask, we conclude junction insulation, in particular how the film seals around the edge of a junction, plays a large role in the junction quality. $R_{S G} / R_{N}$ was shown to increase by a factor of 2 by solely changing the method of $\mathrm{SiO}_{2}$ deposition. We note, the Al Quadlevel process, combined with the New Test Mask described in this chapter greatly reduced fabrication time from $\sim 1$ month to as little as $\sim 1$ week for POGO test devices compared to the pentalevel process, and lays the foundation to more quickly investigate new SIS trilayer materials, some of which are investigated in Chapter 6 .

For future work, the quadlevel $\mathrm{Al}$ process could be implemented in the junction fabrication for the initial phase of AlN based ALMA Band-6 mixers. As we investigate new AlN nitridation schemes to produce higher quality AlN based trilayer, they may not yet be as well characterized in terms of obtaining an accuracy in $R_{N} A$ compared 
to $\mathrm{Al}$-oxide based trilayer. With this in mind, the modest $\sim 10 \%$ variance in junction resistance for $1.7 \mu \mathrm{m}$ junctions (which modestly increases to $\sim 15 \%$ for $1.0 \mu \mathrm{m}$ junctions assuming a similar variance in junction diameter) across a $50 \mathrm{~mm}$ sample may be advantageous, as it allows researchers to cherry pick mixers with impedances which provide better coupling to their receiver setups. 


\section{Chapter 5}

\section{In Situ Spectroscopic Ellipsometry of Barrier Formation}

\section{$5.1 \quad$ Introduction}

The properties of thin films such as: stress, conductivity, dielectric constant, and composition vary across deposition conditions, and their characterization is an integral part of nano and microelectronics. The most fundamental property of a thin film is its thickness, as both the properties of the film itself, and that of the device it is incorporated are often a function of film thickness. For SIS devices, the tunneling probability through the barrier layer is exponentially dependent upon barrier thickness, and its precise knowledge, or at least repeatability, is required for realization of SIS junctions of proper impedance.

Mechanical methods, such as atomic force microscopy (AFM) and profilometry for determination of a thin film's thickness do exist, but require patterning of the film in question and are generally impractical for the angstrom resolution required for characterization of tunnel barriers. Alternative methods such as cross sectional TEM offer atomic resolution and give valuable insight to the nature of both the thin 
film and interfaces, but are destructive processes which require careful preparation of a sample, and time scales from deposition to measurement are on the order of weeks. Typical barrier layers for SIS devices are on the order of $1 \mathrm{~nm}$, prepared in vacuum, and an exposure to atmosphere would cause the rapid growth of oxides. For these reasons, a non destructive, in situ measurement of sub-nanometer resolution is desired; we therefore focus our attention on optical methods.

Optical methods rely on the interaction of light with the film in question, and can be split in to two general categories, interferometric and ellipsometric. Interferometry relies on the interference between the phases of light caused by different path differences as they reflect from opaque topography, or from both the surface and interface of a transparent film. Ellipsometry relies not on interference, but on the change in polarization of light as it reflects at non-normal angles from interfaces of differing refractive indices, as such, its accuracy does not depend on wavelength. Ellipsometry is over a century old technique and in its modern form has been experimentally shown to resolve thicknesses of thin films down to sub-monolayer accuracies [60,61,62]. Due to its sub-monolayer resolution in thickness and its ability to be preformed in situ, ellipsometry is our preferred method for monitoring the growth of, and characterization of thin films produced via vacuum deposition techniques. Both single wavelength ellipsometry (SWE) and spectroscopic ellipsometry (SE) and their applications and limitations with respect to monitoring the formation of tunnel barriers during SIS trilayer deposition are discussed, and a new quantitative SWE model is developed and tested as part of this work. 


\subsection{Ellipsometric Determination of Film Proper- ties}

In this section we review the basic principles of electromagnetic waves and their interactions with mediums of varying refractive indices. Building on these principles, we derive the necessary ellipsometric equations relating the thickness and optical properties of thin film(s) to the measured changed in polarization. Previous models of AlN growth are discussed and a new model for AlN growth is proposed. The effects of common experimental variations on measurement error are evaluated, and a self-calibrated iterative measurement scheme is proposed. The following texts, from which the equations and models in this chapter are derived, are recommended for a more in depth review $[58,63,64,65,66,67,68,69]$.

\subsubsection{Electromagnetic Waves and Polarization}

Light is a transverse electromagnetic wave, having a magnetic field vector perpendicular to a electric field vector, both perpendicular to the direction of propagation. Because the magnetic field vector is not independent of the electric field vector, we limit our focus the E-field propagating along a straight vector, $\hat{z}$ :

$$
E=E_{0} e^{i(k z-\omega t)}
$$

where $k$ is the wave number, $\omega$ is the angular frequency, and $E_{0}$ is the magnitude of the wave. The wavenumber $k$ describes the propagation of the field and is defined as:

$$
k=\frac{2 \pi \tilde{n}}{\lambda_{0}}
$$

where $\lambda_{0}$ is the free space wavelength, and $\tilde{n}$ is the complex index of refraction defined as: 


$$
\tilde{n}=n+i \kappa
$$

substituting $\tilde{n}$, equation 5.1 becomes:

$$
E=E_{0} e^{i(2 \pi \tilde{n} z / \lambda-\omega t)}=E_{0} e^{i 2 \pi n z / \lambda} e^{-2 \pi \kappa / \lambda} e^{-i \omega t}
$$

From 5.4, the real part of the index of refraction, $n$, describes the speed of the wave relative to its free space value, and the imaginary part, $\kappa$, often referred to as the extinction coefficient describes the attenuation of the the wave's amplitude. However, detectors (including our human eye) observe not the magnitude of the electric field, but its square representing the power flux or intensity, $I=E^{2}$. Noting 5.4 , the decay in intensity can be described as:

$$
I(z)=I_{0} e^{-\alpha z}
$$

where $\alpha$ is the more commonly used attenuation coefficient describing power loss during propagation:

$$
\alpha=\frac{4 \pi \kappa}{\lambda}
$$

When light approaches a medium of differing index of refraction, part of the wave passes through the interface and part is reflected as shown in Figure 5.1. Taking into account continuity at the interface, the angles of reflection $\left(\theta_{r}\right)$, incidence $\left(\theta_{i}\right)$, and transmission $\left(\theta_{t}\right)$ are related by the familiar Snell's law:

$$
\begin{gathered}
\theta_{i}=\theta_{r} \\
\tilde{n}_{1} \sin \left(\theta_{i}\right)=\tilde{n}_{2} \sin \left(\theta_{t}\right)
\end{gathered}
$$




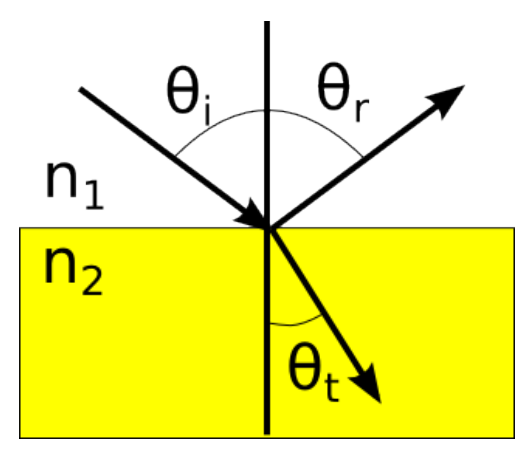

Figure 5.1: Reflection and refraction of light at an interface between two regions of different indices of refraction.

Building upon Snell's law to calculate the relative amplitudes of the reflected and transmitted waves, we need to take into account the polarization of the light. As mentioned each photon contains a E-field vector. If the E-field vectors of the photons at a particular time and place are aligned the light is said to be polarized, else, for random alignment, the light is said to be unpolarized. To visualize the 3 types of polarization, let use resolve the E-field into two orthogonal components, $E_{x}=$ $E_{x 0} e^{i\left(k z-\omega t+\theta_{x}\right)}$ and $E_{y}=E_{y 0} e^{i\left(k z-\omega t+\theta_{y}\right)}$, for a wave propagating in the $z$ direction. If both components are in phase, $\theta_{x}-\theta_{y}=0$, they will trace out a straight line in space or time, producing linearly polarized light. However, if the $x$ and $y$ components have a phase difference of \pm 90 degrees, the E-field vector will trace a circle producing circularly polarized light. Linearly and circularly polarized light are specific cases of the general elliptically polarized light, as when $\theta_{x}-\theta_{y} \neq 0$ or $90^{\circ}$ the E-field traces a ellipse. Visually, all three types of polarization are shown in Figure 5.2.

\subsubsection{Reflections from Thin Films}

When light reflects or refracts at an interface, the angles of reflection and transmission are related by the refractive indices. However, the reflection and transmission coefficients, describing the magnitude of the reflected or transmitted waves respectively, depend on the polarization of the E-field owing to continuity of boundary condi- 

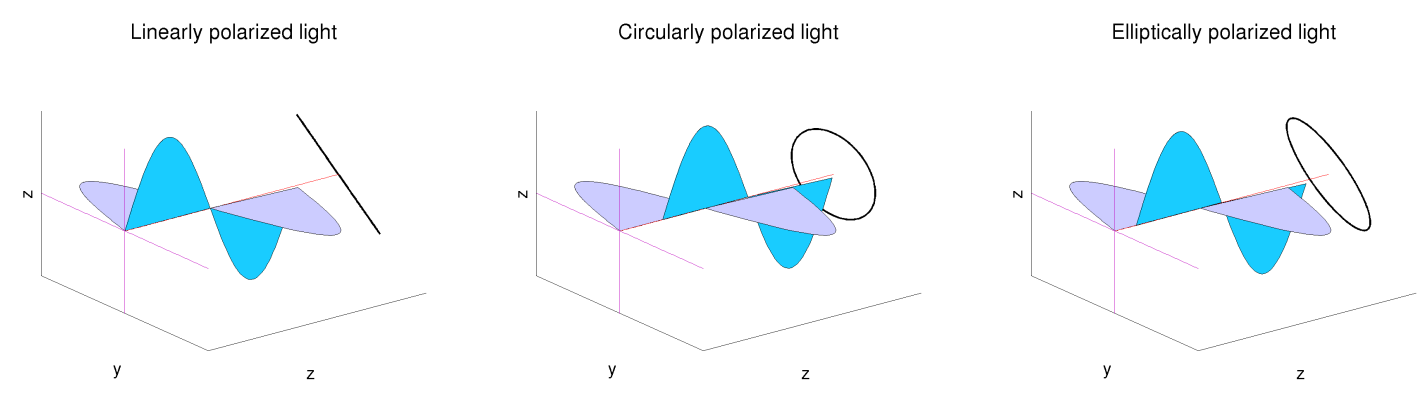

Figure 5.2: Left: Linearly polarized light when $\theta_{x}-\theta_{y}=0$, and the E-field oscillations trace a line. Middle: Circularly polarized light when $\theta_{x}-\theta_{y}=90^{\circ}$ and the E-field oscillations trace a circle. Right: The general case of elliptically polarized light when $\theta_{x}-\theta_{y} \neq 0$ or $90^{\circ}$, the oscillating E-field traces an ellipse.

tions at the surface. If we resolve the E-field into two components, parallel (p-wave) and perpendicular (s-wave) to the plane of incidence, the reflection and transmission coefficients are described by the Fresnel Equations:

$$
\begin{gathered}
r_{12}^{p}=\frac{E_{o r}^{p}}{E_{o i}^{p}}=\frac{k_{2} \cos \left(\theta_{i}\right)-k_{1} \cos \left(\theta_{t}\right)}{k_{2} \cos \left(\theta_{i}\right)+k_{1} \cos \left(\theta_{t}\right)} ; t_{12}^{p}=\frac{E_{o t}^{p}}{E_{o i}^{p}}=1+r_{12}^{p} \\
r_{12}^{s}=\frac{E_{o r}^{s}}{E_{o i}^{s}}=\frac{k_{1} \cos \left(\theta_{i}\right)-k_{2} \cos \left(\theta_{t}\right)}{k_{1} \cos \left(\theta_{i}\right)+k_{2} \cos \left(\theta_{t}\right)} ; t_{12}^{s}=\frac{E_{o t}^{s}}{E_{o i}^{s}}=1+r_{12}^{s}
\end{gathered}
$$

where the respective angles are determined by Snell's Law, $p$ stands for parallel and $s$ stands for perpendicular with respect the the plane of incidence.

To see how these equations can be useful for thickness measurement, take the case of a thin film of with index of refraction $\tilde{n}_{1}$ and thickness $d_{1}$ on a thick substrate of index of refraction $\tilde{n}_{2}$ in a medium with index of refraction $\tilde{n}_{0}$ as shown in Figure 5.11. The light reflected off the thin film's surface has reflection coefficient $r_{01}$, the light that is transmitted through the thin film vacuum interface, reflects off the substrate, and emerges from the thin film vacuum interface, has a reflection coefficient $t_{01} r_{12} t_{10} e^{i 2 k_{1} d_{1} \cos \left(\theta_{t}\right)}$ with respect the thin film vacuum interface. The $t_{01} r_{12} t_{10}$ term takes into account the transmissions through the thin-film vacuum interface and reflections from the thin-film substrate interface, while the $e^{i 2 k_{1} d_{1} \cos \left(\theta_{t}\right)}$ term takes into 


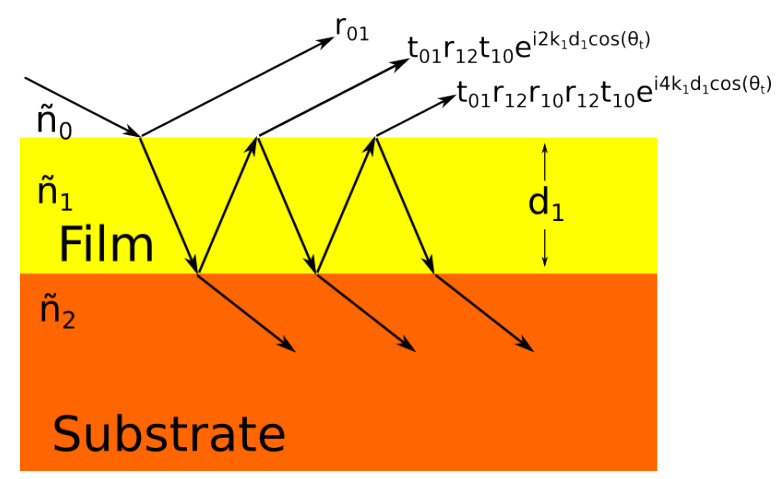

Figure 5.3: This figure shows some of the possible trajectories as light is incident on the surface of an optically thin film on an optically thick substrate. Light can either be refracted or reflected at each interface, and we assume the substrate is sufficiently thick that once light enters, it decays before it reaches another interface.

account phase change and attenuation as the light travels through the thin film. Keeping track of all possible trajectories and summing them, we can fully describe the total reflection from the surface of a sample with a single thin film in terms of the angel of incidence, the thin film's thickness, and all optical constants:

$$
R=r_{01}+t_{01} r_{12} t_{10} e^{j 2 k_{1} d_{1} \cos \left(\theta_{t}\right)}+t_{01} r_{10} r_{12}^{2} t_{10} e^{i 4 k_{1} d_{1} \cos \left(\theta_{t}\right)}+t_{01} r_{10}^{2} r_{12}^{3} t_{10} e^{i 6 k_{1} d_{1} \cos \left(\theta_{t}\right)}+\ldots
$$

which can be simplified to:

$$
R=\frac{r_{01}+r_{12} e^{i 2 k_{1} \cos \left(\theta_{t}\right)}}{1+r_{01} r_{12} e^{i 2 k_{1} \cos \left(\theta_{t}\right)}}
$$

Noting 5.12, the total reflection from an infinite number of films can be calculated through proper book keeping.

\subsubsection{The Ellipsometer}

We have shown that the total reflection coefficients for the p-waves and s-waves are a function of the angle of incidence, optical constants, and film thicknesses. The phase 


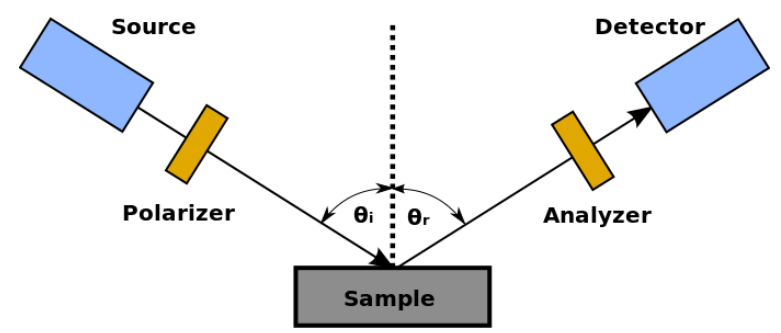

Figure 5.4: Simplified block diagram of an ellipsometer setup. Light is emitted from a source, and polarized to a know polarization. After reflecting from a sample surface, the polarization of the light is determined through passing through a rotating polarized filter before reaching a detector.

between the p-waves and s-waves and the ratio of their magnitude change, define the shape of the ellipse their combined E-field vector traces in time. We define the change in phase difference between the p-wave and s-wave after reflection as $\Delta$, and the ratio of their respective magnitudes as:

$$
\frac{\left|R^{p}\right|}{\left|R^{s}\right|}=\tan (\Psi)
$$

It is sometimes confusing to new readers to why $\tan (\Psi)$ is used instead of a constant term, but we note this ratio can vary from 0 to $\infty$; however, when this ratio is expressed in terms of $\tan (\Psi), \Psi$ conveniently varies from 0 to $90^{\circ}$. This leads us to define the complex ratio of total reflection $\rho_{r}$ in terms of $\Delta$ and $\Psi$, often referred to as the fundamental equation of ellipsometry [67, 65]:

$$
\rho_{r} \equiv \frac{R_{p}}{R_{s}}=\frac{\left|R_{p}\right| e^{i k_{p}}}{\left|R_{s}\right| e^{i k_{s}}}=e^{i \Delta} \tan (\Psi)
$$

The ellipsometer, as shown in Figure 5.4, reflects light of a known polarization (usually linearly polarized) from a sample surface, and measures the the complex ratio, $\rho_{r}$, of the reflected light, which is often expressed in terms of $\Delta$ and $\Psi$. From the measurements of $\Delta$ and $\Psi$, the optical constants and/or thickness of a film can be determined indirectly through an iterative approach, using a least squares regression on the free variables of a mathematical model of the sample. 


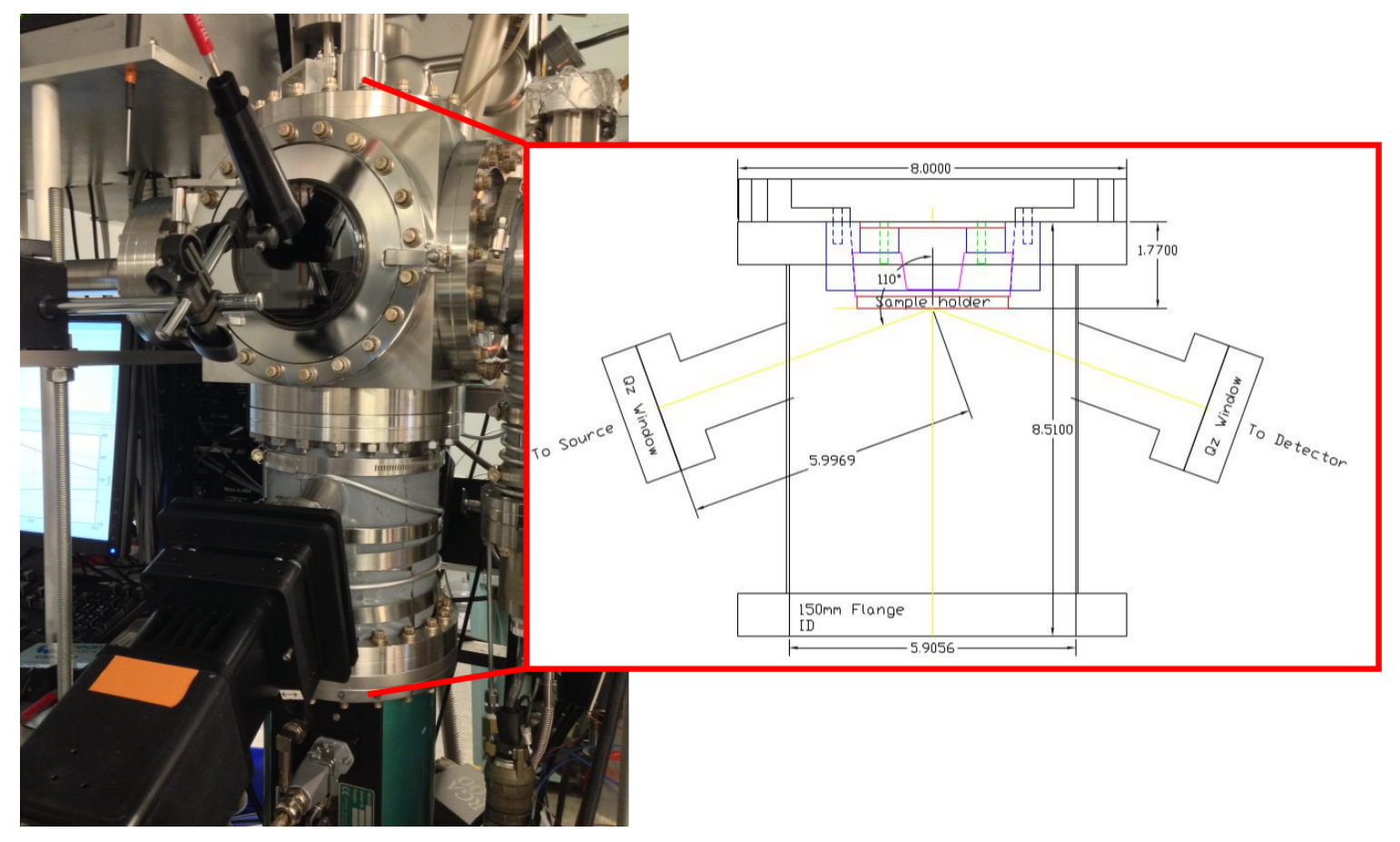

Figure 5.5: A view of our nitridation chamber along with the cross sectional CAD drawing showing the position our samples as they rest on the holder, and the two optical feedthroughs to which the source and detector are attached, providing a 70 degree angle of incidence. 


\subsubsection{Single Wavelength vs Spectroscopic Ellipsometry}

Our group first demonstrated the use of in situ SWE ellipsometry to provide qualitative analysis of AlN growth via ICP [24]. While a SWE approach allowed us to investigate how process parameters affected AlN growth rates, there are fundamental limitations to SWE. For in situ SWE applications, the angle of incidence is fixed, and because data are collected at a single wavelength, regressive analysis can only fit a single parameter, such that the analysis is limited to a single film where the optical constants are well defined. At a single wavelength, changes in optical constants and thickness can alter $\Delta$ and $\Psi$ in an ambiguous fashion, and since the optical properties of thin films vary from their bulk values, an error in calculated thickness can result from incorrectly assumed optical constants. Furthermore, our AlN films are grown through the use of an $\mathrm{Al}$ overlayer process, where a thin 5-8 $\mathrm{nm} \mathrm{Al} \mathrm{film} \mathrm{is} \mathrm{deposited}$ on an optically thick $\mathrm{Nb}$ layer and exposed to a nitrogen ICP to form AlN on the surface. Inherently, a correct model relies on the analysis of both the AlN layer, which increases in thickness, and the underlying Al layer, which is consumed during ICP AlN growth. The optical penetration depth of $\mathrm{Al}$ is around $7 \mathrm{~nm}$, which is on the order of our film thickness, and it must be taken into account for accurate analysis $[65,70]$.

SE on the other hand, measures the change in optical constants across multiple wavelengths, increasing the number of data points for regressive analysis. Furthermore, the optical constants of films often follow well defined dispersion characteristics. While two films may have similar optical constants at a single frequency, they often follow different dispersion relationships, removing ambiguity which may occur from SWE. Noting the increased amount of data points and the ability to define layer optical constants through wavelength dependent dispersion relationships, com-

plex material stacks containing more than one thin film can be analyzed through regressive fitting of multiple parameters. The next section introduces the dispersion 
relationships and parameters used in building our SE model to in situ monitor AlN growth.

\subsection{Ellipsometric Model and Parameters}

\subsubsection{Modeling Dispersions of Thin Films}

When an EM wave passes through a material, its E-field exerts a force on charged particles. If the charged particles are bound, such as electrons bound to a nucleus or ion's in a lattice, an analogous mechanical system is mass on a spring and the response should follow that of an oscillator. Oscillators are often the starting point to building a dispersion model, and often a sum of oscillators is used to capture the effects of multiple dispersion mechanisms in a single film. One of the most basic is the classical Lorentz oscillator which can be derived from solving the second order differential equation of its mechanical analog [65]:

$$
\tilde{\epsilon}=\epsilon_{\infty}+\frac{4 \pi N e^{2}}{m} \frac{1}{\left(\omega_{0}^{2}-\omega^{2}-i \Gamma \omega\right)}
$$

were $\tilde{\epsilon}=(n+i \kappa)^{2}$ is the complex dielectric constant, $N$ represents the density of oscillators, $e$ is the fundamental charge, $m$ is the mass of the oscillator, $\omega_{0}$ is the resonant frequency of oscillation, and $\Gamma$ is a broadening term. Other variations of oscillators are often used such as the Harmonic and Gaussian, but are not used in our model and are covered elsewhere [64].

\subsubsection{Metallic Films}

The free electrons in metal films are often modeled using the Drude model, which is a modification of the Lorentz oscillator, where the resonant frequency is set to zero to account for unbound charge carriers. The resulting Drude term is: 


$$
\tilde{\epsilon}=\epsilon_{\infty}-\frac{\omega_{p}^{2}}{\omega^{2}-i \Gamma}
$$

where $\omega_{p}=\sqrt{4 \pi N e^{2} / m_{e}}$ is the plasma frequency of the metal [66]. Noting this, the Drude model relates to the metals resistivity $\rho_{m}$ and mean scattering time $\tau_{m}$ through the free electron model:

$$
\begin{gathered}
\rho_{m}=\frac{\Gamma}{\epsilon_{\infty} \omega_{p}^{2}} \\
\tau_{m}=\frac{\hbar}{\Gamma}
\end{gathered}
$$

SE measurements can been used to indirectly measure these quantities for thin films [71].

\subsubsection{Insulating Films}

The real part of the refractive index of insulating films are found to follow the empirical Cauchy's Equation for photon energies below the bandgap of the material:

$$
n(\lambda)=C_{0}+\sum_{n=1}^{\infty} \frac{C_{n}}{\lambda^{2 n}}
$$

where the $C$ terms are called the Cauchy Parameters. While sufficient for large bandgap insulators probed in the visible spectrum, the Cauchy Equation does not capture absorption by the film, and is insufficient by itself for ellipsometric measurements approaching the bandgap of the material where absorption processes cannot be neglected. For AlN, the bandgap corresponds to a wavelength of $200 \mathrm{~nm}$, which is in the lower wavelength limit of many commercially available ellipsometers. Noting this, a separate model for the extinction coefficient is used. The simplest form is to use another Cauchy Equation to model the dispersion of the extinction coefficient, 
however, we prefer to use the Urbach Equation:

$$
\kappa(\lambda)=C_{1} e^{C_{2}\left(E_{p}-E_{0}\right)}
$$

as it has been shown AlN demonstrates an exponential relationship in its extinction coefficient as photon energies $E_{p}$ approach its bandgap $E_{0}[72]$.

\subsubsection{Surface Roughness}

Non-epitaxial thin films may have a physical roughness caused by grain boundaries and non-uniform growth at nucleation points. A common approach used to model surface roughness is to replace the rough interface with a film that has an index of refraction composed of a mixture of two layers and a thickness equal to that of the RMS roughness as shown in Figure 5.6. A simple averaging of the layers, $\tilde{n}_{e f f}=f_{0} \tilde{n}_{0}+f_{1} \tilde{n}_{1}$, where $f$ denotes the fractional volume occupied by the film in the roughened layer, is a mathematically simple approach, but it found to be inaccurate. Instead, often the Bruggeman Effective Medium Approximation (EMA) is used[65]:

$$
f_{0} \frac{\tilde{n}_{0}-\tilde{n}_{e f f}}{\tilde{n}_{0}+2 \tilde{n}_{e f f}}+f_{1} \frac{\tilde{n}_{1}-\tilde{n}_{e f f}}{\tilde{n}_{1}+2 \tilde{n}_{e f f}}=0
$$

where $f_{0}$ is the fractional volume of film $0, f_{1}$ is the fractional volume of film 1, $\tilde{n}_{0}, \tilde{n}_{1}, \tilde{n}_{e f f}$ are the refractive indices of film 0,1 and the effective index of the rough layer respectively. For surface roughness, the properties of film 0 are taken to be vacuum. We note the Bruggeman EMA assumes uniform spherical inclusions and that the thickness of the effective film is equal to the RMS surface roughness. While this assumption is not always physically true, the Bruggeman EMA works extremely well for roughnesses much less than the wavelength of the light, and the surface roughness of $\mathrm{Al}$ wetted $\mathrm{Nb}$ films is typically less than $1 \mathrm{~nm}[69,73]$. 


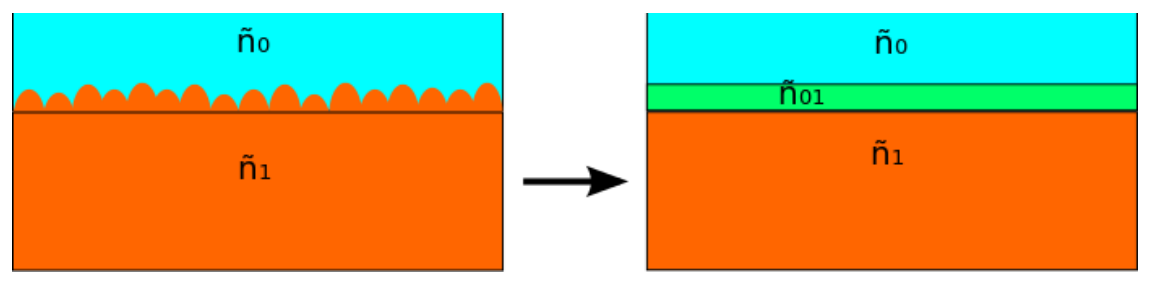

Figure 5.6: Model demonstrating the concept of an EMA. Left: Rough surface of film with index of refraction $\tilde{n}_{1}$ interfacing with region with index of refract $\tilde{n}_{0}$. Right: The ellipsometric EMA model were the surface region is replaced by film of effective index of refraction $\tilde{n}_{01}$.

\subsection{Experimental Setup and Proposed Model}

A J. A. Woollam Co. M-2000U® Ellipsometer was installed in the nitridation chamber of the deposition system described in Chapter 3. The angle of incidence is fixed at 70 degrees through a pair of 2" conflat optical feedthroughs, and the sample is suspended in the plane of incidence by a fixed stainless steel holder as shown in Figure 5.5. A notable feature of the M-2000U ellipsometer is that it contains 470 individual CCD detections which capture all wavelengths from 235-1000 nm simultaneously, allowing for real time in situ SE data collection. Our models were developed and analyzed using J. A. Woollam Co. CompleteEASE® software package. CompleteEASE® allows a user to define an arbitrary stack of materials to create an ellipsometric model, the materials' optical constants can be defined as tabulated data or mathematical dispersion relationships. Any and all constants of the mathematical models can be fixed or set as free variables with bounds fitted to the recorded $\Delta$ and $\Psi$ values through a regressive least-squares approach. In addition to fitting model parameters, the software package can be used to generate ideal $\Delta$ and $\Psi$ data from a user defined model with fixed variables. Generation of ideal synthetic data are useful for evaluating how a model's variables may affect the iterative data fitting and introduce error.

Because variables are fit using a least squares regression, a figure of merit to determine the goodness of fit is required. The root mean squared error (called MSE 
for historical reasons) is used:

$$
M S E=\sqrt{\frac{1}{3 n-m} \sum_{i=1}^{n}\left[\left(N_{E_{i}}-N_{G_{i}}\right)^{2}+\left(C_{E_{i}}-C_{G_{i}}\right)^{2}+\left(S_{E_{i}}-S_{G_{i}}\right)^{2}\right]} \times 1000
$$

where $E$ represents experimental data, $G$ represents generated data from model fit, $n$ is number of wavelengths $m$ is number of fit parameters, $N=\cos (2 \Psi), C=$ $\sin (2 \Psi) \cos (\Delta)$, and $S=\sin (2 \Psi) \sin (\Delta)[64]$. While goodness of fit tells us how well a model matches its experimental data, we note a low MSE does not guarantee a physically correct model, only a good mathematical fit. As such, intelligent development and verification is required when creating and using a model.

For extremely thin $(\sim<5 \mathrm{~nm})$ films, the thickness of a single transparent film is approximately proportional to the change in $\Delta$ of the surface, and changes in $\Psi$ are often small and difficult to measure. Our original SWE tool calculated a thickness by monitoring the change in $\Delta$ from the surface of our Al overlayer during nitridation. To test the validity of building a more complex SE model, we first needed to test the ability to monitor changes in both $\Delta$ and $\Psi$ for our particular films of interest. Figure 5.7 shows the measured $\Delta$ and $\Psi$ values recorded from a $6 \mathrm{~nm} \mathrm{Al}$ overlayer deposited on a $165 \mathrm{Nb}$ film before an after being exposed to a $200 \mathrm{~W}, 5.0 \mathrm{mT}$ ICP for 40 minutes, which is on the order of our typical nitridation times. While the change in $\Psi$ is less than that of $\Delta$, a clear change in both $\Delta$ and $\Psi$ is observed, and should allow us to build a more physically accurate SE model taking into account changes to both $\Delta$ and $\Psi$.

We will first compare two ellipsometric growth models of a transparent film (either $\mathrm{AlN}$ or $\mathrm{Al}$-oxide) grown on a thin $\mathrm{Al}$ overlayer, deposited on an optically thick $\mathrm{Nb}$ substrate. The first model is an SE extension of the SWE model. The wafer is scanned after the Al overlayer deposition, and its optical constants are saved and used as the substrate. On top of the substrate, a single AlN (or Al-oxide) film 

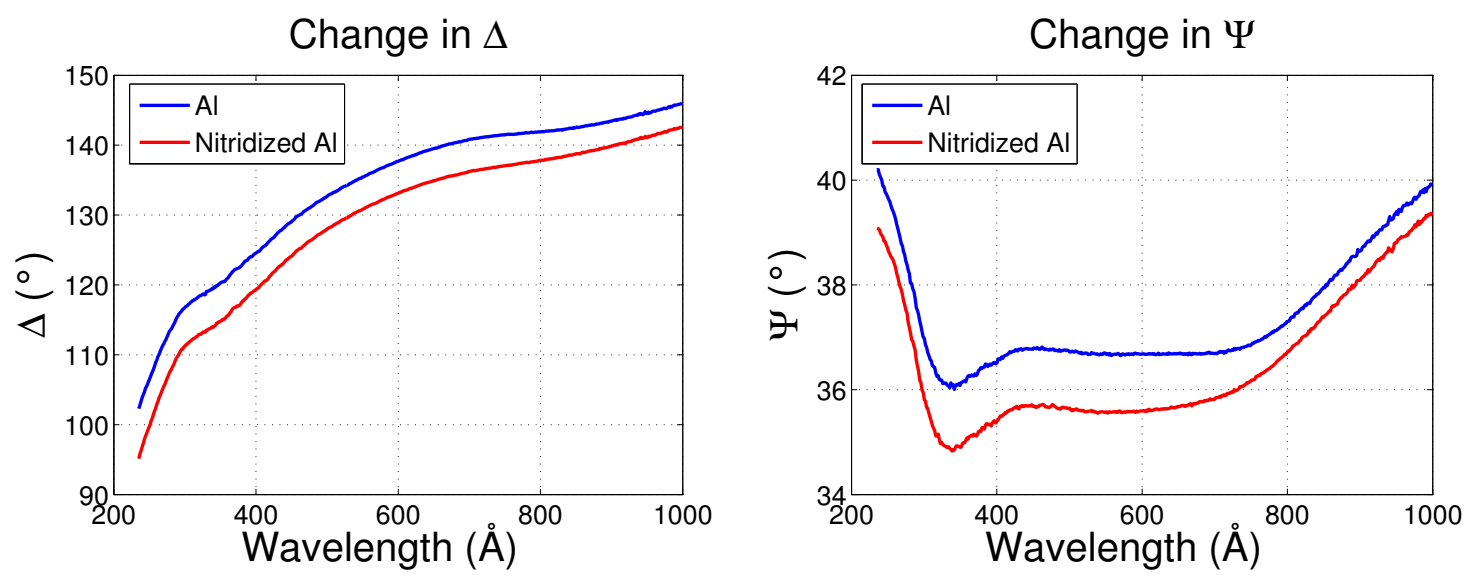

Figure 5.7: Change in $\Delta$ and $\Psi$ of an $6 \mathrm{~nm} \mathrm{Al}$ overlayer on $165 \mathrm{~nm} \mathrm{Nb}$ film after being exposed to a $200 \mathrm{~W}, 5.0 \mathrm{mT}$ ICP for 40 minutes.

is modeled and monitored for growth. The second model used is more physically accurate, consisting of a thin AlN (or Al-oxide) film on a thin $\mathrm{Al}$ overlayer on an optically thick $\mathrm{Nb}$ substrate. Both the increasing AlN (or Al-oxide) film thickness as well as the decreasing $\mathrm{Al}$ overlayer thickness are monitored during growth. The CompleteEASE® software contained no off-the-shelf AlN dispersion model and an optically thin Al film's properties differ from that of bulk Al. The creation of both of thin $\mathrm{Al}$ and $\mathrm{AlN}$ dispersions are discussed in the following sections.

\subsubsection{Modeling Al and AlN Films}

The aluminum overlayer film was modeled using a sum of Lorentz and a single Drude oscillator. To fit the model, $3 \mathrm{Al}$ overlayers ( $\sim 6 \mathrm{~nm}$ each) were deposited in situ on a single silicon wafer with a $300 \mathrm{~nm}$ thick thermally grown oxide. After the first overlayer was deposited, the wafer was quickly transfered to the ellipsometer stage and scanned, the resulting $\Delta$ and $\Psi$ values were saved. This was repeated a total of 3 times on the same sample to build a final thickness of $3 \mathrm{Al}$ overlayers. We note that oxidation of the $\mathrm{Al}$ film between the deposition of each overlayer could affect our model, however, the partial pressure of water in the nitridation chamber was $<1$.0e- 8 Torr and each scan in between the $\mathrm{Al}$ overlayer depositions took 1-2 minutes. Noting 
the background oxidation plots from Figure 3.3 on page 32, appreciable oxidation is believed not to have taken place. We chose to use 3 overlayers, as it allowed us to iteratively build and check the validity of our model knowing $t_{3}=3 t_{1}$ and $t_{2}=2 t_{1}$ for 3 and 2 overlayers.

To iteratively fit the parameters of our model to our data, we did the following process. Before $\mathrm{Al}$ deposition, the $\mathrm{Si}$ wafer with thermally grown $\mathrm{SiO}_{2}$ was scanned and both the thickness of the $\mathrm{SiO}_{2}$ film and offset angle of the wafer were fit to a well characterized model provided by J.A. Woollam. An Al film model was created using a sum of 4 Lorentz oscillators and one Drude oscillator, creating a model stack of $\mathrm{Si}$ (substrate) $/ \mathrm{SiO}_{2} / \mathrm{Al}$, where the properties of the $\mathrm{SiO}_{2}$ film were determined from the first scan and fixed. The starting point of the free variables of the Al model were taken from the bulk resistivity and scatter time of $\mathrm{Al}$ for the Drude oscillator, and the starting point of the variables for the Lorentz oscillators were taken from a 'bulk' Al Lorentzian model provided by J.A. Woollam. CompleteEASE® allows a user to best fit one of more variables of a model to recorded data. The variables were best fit one at a time to the data from the scan of the single aluminum overlayer. This process was repeated, cycling through the free variables, until the MSE of the fit was below 10. The process was then repeated for the data from the scan of 2 (and then 3) aluminum overlayers, except the initial thickness of the $\mathrm{Al}$ film in the model was guessed to be twice (and then triple) that determined from the single layer.

This process was repeated back and forth between the data from 1, 2, and $3 \mathrm{Al}$ overlayers until the same $\mathrm{Al}$ model produced MSE values below 10 for each, and we obtained the dispersion relationships shown in Figure 5.8 which follow a qualitatively similar dispersive trend (with a similar range for $n$ from $~ 0.4-2.1$ ) to published data for $\mathrm{Al}$ films $[74,75]$. To test the validity of our model, the resistivity was set as a free variable for all three thicknesses using the Drude oscillator component of our model and best fit. The resistivity was found to decrease slightly with increasing $\mathrm{Al}$ 

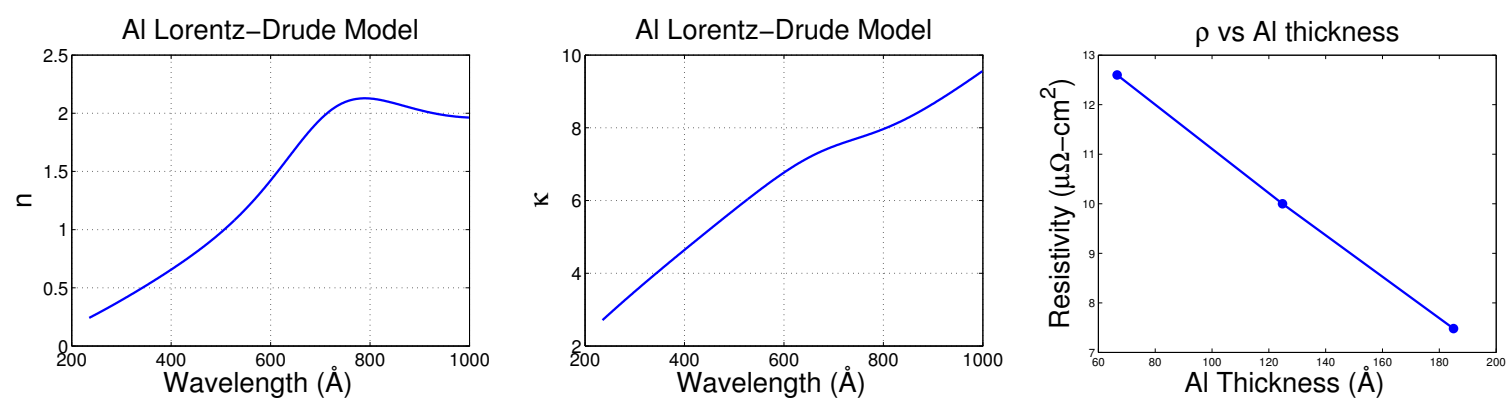

Figure 5.8: Left: Index of refraction of our $\mathrm{Al}$ model. Center: Extinction coefficient of our Al model. Right: Variation of resistivity with film thickness as determined by the Drude oscillator in our model. Decrease in resistivity with increasing film thickness is reported in the literature and used to check the validity of our model.

thickness, as expected for thin metallic films. Additionally a four point measurement performed on the sample after deposition produced a resistivity of $7.0 \mu \Omega-\mathrm{cm}$ compared to Drude model's $7.5 \mu \Omega-\mathrm{cm}$. Noting this, we believe our dispersion provides a reasonably accurate model for thin $\mathrm{Al}$ films.

To create a model for our AlN films, we reactively sputtered a $50 \mathrm{~nm}$ AlN film in $3.0 \mathrm{mT}$ of pure nitrogen using a $150 \mathrm{~W}$ applied power to the 3" aluminum sputtered gun described in Chapter 3 onto a $300 \mu m$ thick $50 \mathrm{~mm}$ silicon wafer with a native oxide. A silicon wafer with a native oxide was chosen as its optical properties are well known through published data from multi-sample, multi-wavelength, multi-angle studies, and provided a well characterized substrate to determine the optical constants of a single thin film [76]. The AlN film thickness was verified through use of a Dektak profilometer and the optical constants were modeled and fit to the Cauchy and Urbach Equations. The resulting index of refraction and extinction coefficient are shown in Figure 5.9 and follow qualitatively similar dispersive trends with a similar range to published values $(n: \sim 1.8-2.4, \kappa: \sim 0-0.6)$ for AlN films $[72,77]$. These dispersions were used for the AlN films in our model. 

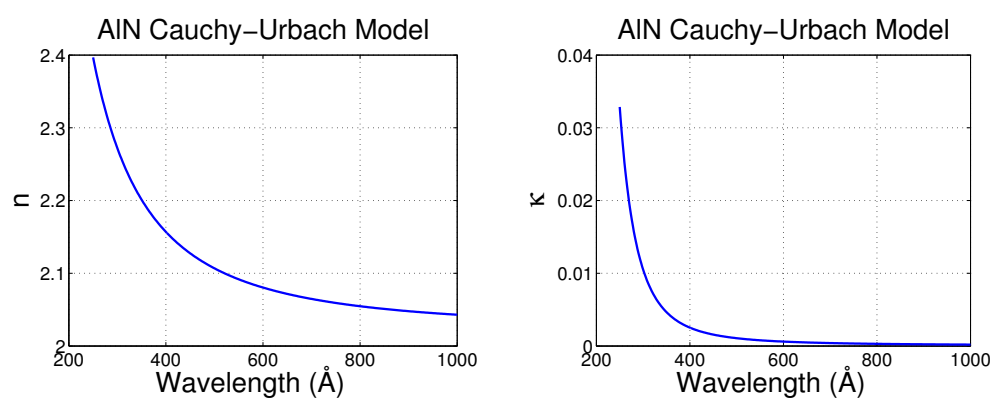

Figure 5.9: Left: Dispersion of the index of refraction of our AlN films described by a three term Cauchy Equation. Right: Dispersion of the extinction coefficient of our AlN films described by the Urbach Equation.

\subsubsection{Sources of error in AlN Thickness}

Errors such as physical angle offsets of our samples, surface roughness, changes in film stress, and incorrect assumptions of Al overlayer thicknesses may manifest themselves as errors in the ellipsometrically determined AlN thickness. We investigated these errors through a series of synthetic experiments using our ellipsometric model and the CompleteEASE® software package. The $\mathrm{Al}$ and $\mathrm{AlN}$ dispersions from the previous section were used to construct a model of AlN growth. The substrate of this model was built upon the tabulated $\Delta$ and $\Psi$ data recorded in situ from a freshly deposited $\mathrm{Nb}$ film on a $\mathrm{Si} / \mathrm{SiO}_{2}$ substrate. On top of the substrate is the $\mathrm{Al}$ and $\mathrm{AlN}$ film models described in the previous sections, producing a $\mathrm{Nb} / \mathrm{Al} / \mathrm{AlN}$ stack as shown in Figure 5.12. Ideal synthetic data were generated for 10, 15, and $20 \AA$ AlN films on top of a nominal $50 \AA \mathrm{Al}$ overlayer. Because $\mathrm{Al}$ is consumed during actual AlN growth, we clarify that the $\mathrm{Al}$ overlayer thickness of $50 \AA$ is the assumed thickness after AlN growth, i.e. the same for each data set. To determine the effects of the above postulated errors, they were introduced into our model one at a time, and the AlN thickness was best fit to the ideal synthetic data using regressive analysis in CompleteEASE®.

In actual experiments, our samples are physically mounted to a sample holder which sits on the ellipsometry stage shown in Figure 5.5. During nitridation a small 
offset in angle from the ideal plane of incidence may occur. Errors in offset angle manifest themselves as errors in the transmission and reflection coefficients shown in equations (5.9-5.10). Angle offsets of $-0.3^{\circ}$ to $0.3^{\circ}$ were investigated. Another source of error may arise from surface roughness of our films, as our ideal model assumes a perfectly flat film. Surface roughness much smaller than the wavelength of the light used can be modeled using a Bruggeman Effective Medium Approximation, described earlier in (5.21). To test the effects of roughness synthetic data were generated assuming a $10 \AA$ RMS roughness and best fit to our model while we varied the assumed RMS roughness from 0 to $20 \AA$. Another error from our model can come from assuming an improper Al thickness if one's model does not simultaneously fit both the $\mathrm{Al}$ thickness and AlN thickness, as is the case with SWE. A $\pm 5 \%$ error in Al overlayer was introduced to our model.

Figure 5.10 shows the resulting error in AlN thickness from these three effects, as well as how the error in AlN thickness affects the $\mathrm{R}_{N} \mathrm{~A}$ values of the trilayer. The error in $\mathrm{R}_{N} \mathrm{~A}$ was calculated making the assumption:

$$
R_{N} A=\alpha e^{\beta t_{A l N}}
$$

where $t_{A l N}$ is the thickness of the AlN barrier layer, and $\alpha$ and $\beta$ are constants found by best fitting this equation to the recorded $\mathrm{R}_{N} \mathrm{~A}$ data from 4 trilayer samples of varying AlN thicknesses. We note that errors in the angle of incidence have the largest effects (can produce errors in $\mathrm{R}_{N} \mathrm{~A}$ of $50 \%$ for as little as a $0.15^{\circ}$ offset) and are taken into account by a run to run calibration scheme described in the following sections. Errors in assumed Al thickness have the smallest effect, but, noting the exponential dependence of $\mathrm{R}_{N} \mathrm{~A}$ to the thickness of the barrier layer, still produce appreciable error. However, our SE model can fit both AlN thickness and Al thickness, reducing this error. Errors in roughness fall somewhere in the middle, generating appreciable error. However, correction for roughness requires the use of an AFM, which is not 

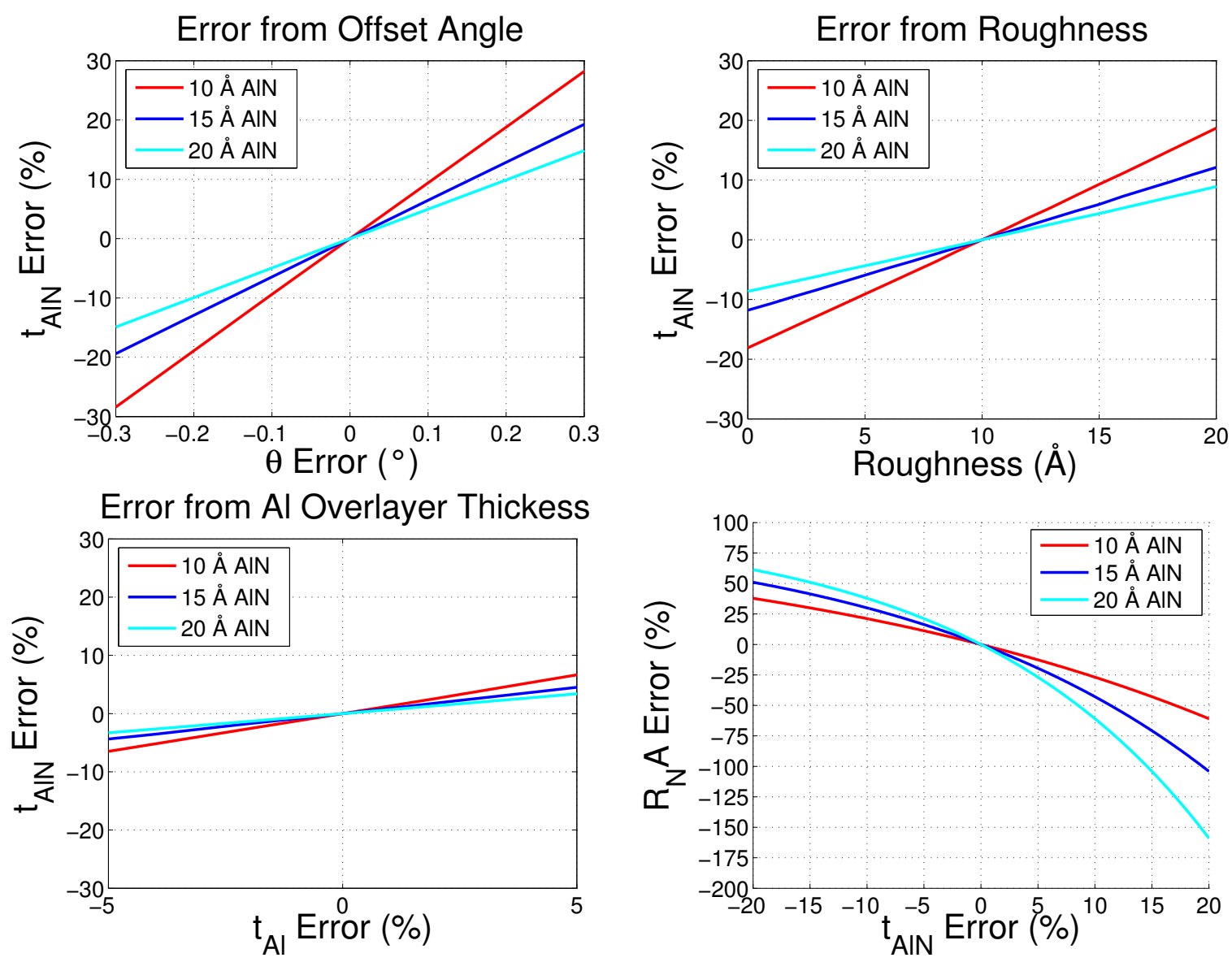

Figure 5.10: Top Left: Error in AlN thickness vs error in offset of the angle of incidence. Top Right: Error in AIN thickness vs surface roughness, for a film with 1 $\mathrm{nm}$ assumed surface roughness. Bottom Left: Error in AlN thickness vs error in $\mathrm{Al}$ overlayer thickness. Bottom Right: Resulting error in trilayer $R_{N} A$ for error in AlN thickness.

readily available in our laboratory. We make the assumption that our $\mathrm{Al}$ wetted $\mathrm{Nb}$ films have low surface roughness, and it remains about constant run to run, but this may remain a source of error.

We also note that optical constants of the thin films may vary with intrinsic film stress, observed by our group for reactively sputtered NbTiN films using SWE [24]. We operate our magnetron sputter guns with conditions to produce dense Nb films with modestly compressive stress. We also note the stress of our Nb films change over the life of the target, even though we periodically adjust the deposition conditions in an attempt to account for these changes. The index of refraction for our $\mathrm{Nb}$ films 

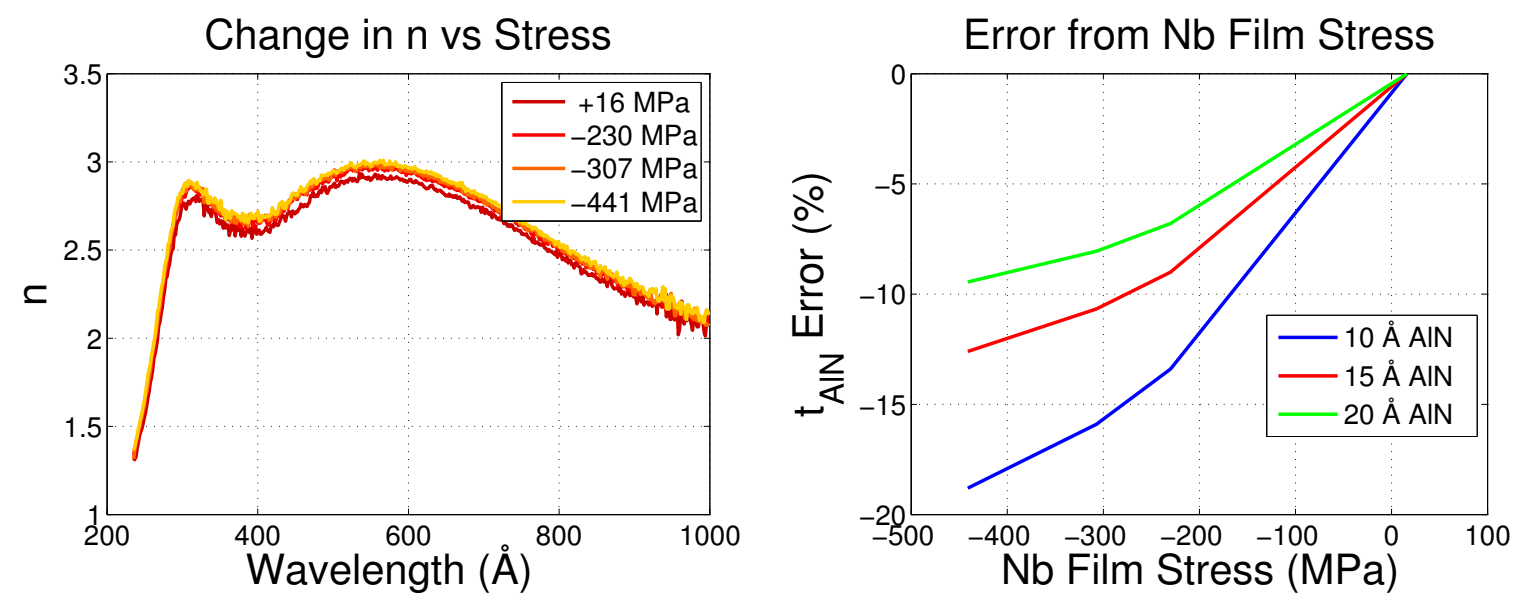

Figure 5.11: Left: Change in index of refraction as a function of film stress. A positive correlation between $n$ and the magnitude of compressive stress is observed. Right: The error in AlN thickness vs film stress when it is not taken into account. The small changes in indices of refraction can produce errors great than $10 \%$.

was recorded for films with stress varying from $+16 \mathrm{MPa}$ (nearly stress free) to -441 MPa (modest compressive stress). Film stress was determined using an FSM thin film stress measurement system described elsewhere [39, 21]. To test the effects of stress on determined AlN thickness, synthetic data were generated for each $\mathrm{Nb}$ stress point using the recorded optical constants. This data were then fed back into our model which assumed a nearly stress free $+16 \mathrm{MPa}$ film. The change in index of refraction as well as the error in AlN thickness due to changes in film stress are shown in Figure 5.11. We note an increase in index of refraction as stress becomes more compressive, as a result, films appear thinner when the film stress is higher than anticipated. The effects of $\mathrm{Nb}$ stress are taken into account through ellipsometric measurements of the M1 Nb film on every run before $\mathrm{Al}$ overlayer deposition, as discussed in the following section.

\subsubsection{Proposed Model for AIN Growth}

The proposed ellipsometric model for AlN growth is shown in Figure 5.12, and the process flow of its implementation showing the various steps is shown in Figure 5.13. 


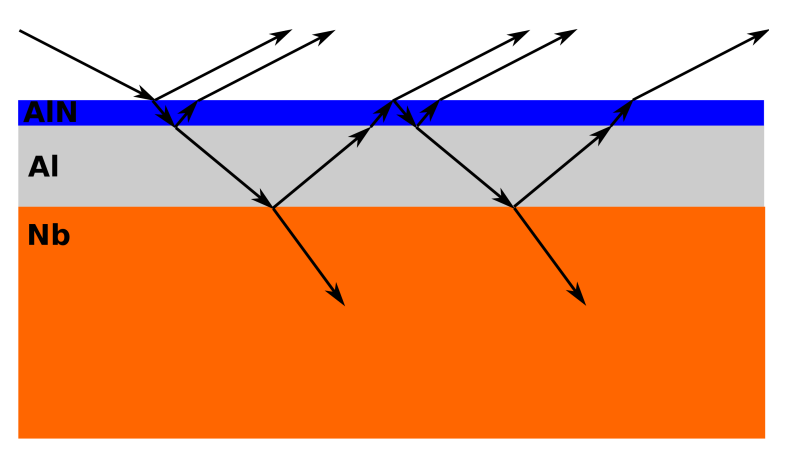

Figure 5.12: A visualization of our ellipsometric model, showing some of the possible trajectories of the light.

For step 1, an $\mathrm{Si} / \mathrm{SiO}_{2}$ substrate is loaded into the system and scanned by the ellipsometer before deposition begins. We note that an offset in the angle of incidence has the largest effect on generating error. $\mathrm{A} \sim 0.1^{\circ}$ error in the angle of incidence is typical across samples and can generate a $\sim 10 \%$ error in thickness. To compensate for angle offset, a run to run calibration using the $\mathrm{Si} / \mathrm{SiO}_{2}$ substrate is performed. $\mathrm{Si}$ and $\mathrm{SiO}_{2}$ are very well defined materials and the resulting data are best fit to an $\mathrm{Si} / \mathrm{SiO}_{2}$ model with the offset in angle of incidence set as a free variable. We note, there is a repositioning error after moving the sample back and forth between the deposition chamber and the nitridation stage, but after repeating this process 5 times with the same $\mathrm{Si} / \mathrm{SiO}_{2}$ sample, the maximum difference in the determined angle on incidence was less than $0.01^{\circ}$. We attribute the low repositioning error to mechanical clamping of our wafer to its holder, the use of a copper dowel pin to precisely align and reposition our translator, and believe the physical offset occurs run to run when the wafer is physically mounted to its holder. Typical errors in angle offset run to run vary by $\pm \sim 0.1^{\circ}$. The corrected angle of incidence, determined from the scan of the $\mathrm{Si} / \mathrm{SiO}_{2}$ substrate, is fed back into our model, to reduce errors from angle offsets.

For step 2, the Nb M1 layer is deposited in the deposition chamber, the sample is brought back to the ellipsometer stage, and the optical constants of the M1 Nb film are measured and tabulated before the Al overlayer deposition. The measured optical constants of the $\mathrm{Nb}$ film reduce error introduced through changes in film stress from 
run to run, and are fed back into our model, along with the offset angle from step 1.

For step 3, after the sample is returned to the deposition chamber, the 5-6 nm $\mathrm{Al}$ overlayer is deposited. The sample is returned to the nitridation stage, and the $\mathrm{Nb} / \mathrm{Al}$ film is scanned by the ellipsometer and fit to our $\mathrm{Nb} / \mathrm{Al} / \mathrm{AlN}$ model, into which both our angle offset, and tabulated $\mathrm{Nb}$ constants have been fed. We know at this point, the AlN film thickness is zero, the Al thickness as well as a few of the model parameters are set as free variable and best fit, setting a zero point for our AlN film and these parameters are fed back into the model. At this point, we have a $\mathrm{Nb} / \mathrm{Al}$ stack and a model to fit our data, self-calibrated run to run with data for the $\mathrm{Nb}$ film, Al film, and angle offset.

For step 4, only the $\mathrm{Al}$ and $\mathrm{AlN}$ thickness are set as free variables, the ICP is ignited, and the wafer is lowered onto the ellipsometry stage to monitor AlN growth in real time. We note, the wafer is removed from the nitridation stage while the ICP is ignited, as a high pressure spike is required, and we allow the ICP to stabilize before AlN growth. The ICP remains lit while we return the sample to the nitridation stage, and some initial AlN growth occurs during this time frame (a few seconds) as the wafer is lowered into place. However, because we first scanned our wafer without AlN, we have a zero reference point which allows us to capture the amount of growth that occurs as we lower the wafer onto the nitration stage. The amount of initial growth is observed on our AlN growth curves as the non-zero initial AlN thickness as shown in Figure 5.14, which we note should be more pronounced for higher growth rate $\mathrm{ICP}$ conditions. 


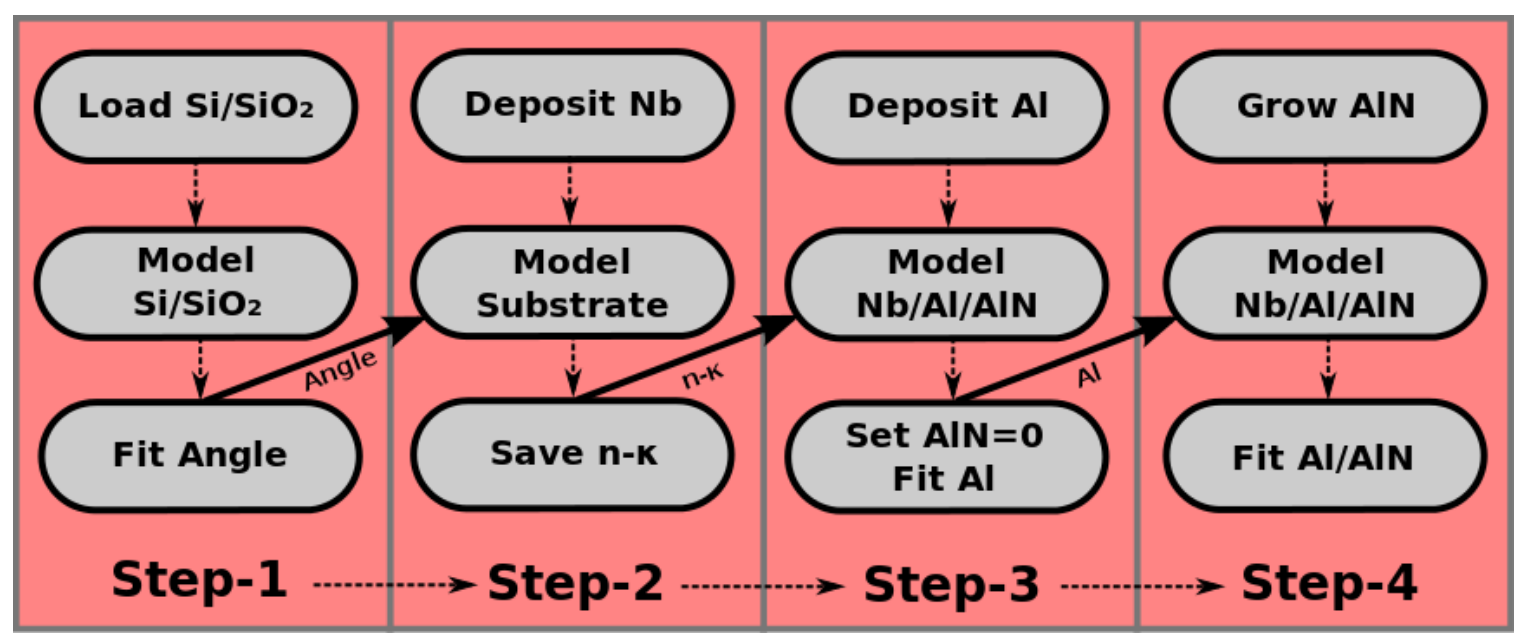

Figure 5.13: Process flow chart for monitoring AlN growth via in situ SE. Steps 1-4 are shown from left to right. For each step from top to bottom is the step name, model used, and parameters adjusted in the model.

\subsection{Results and Conclusions}

\subsubsection{Comparisons of Models}

To verify the validity of our model, we first applied it to the well understood and behaved process of oxidation of Al overlayers. Our AlN Cauchy-Urbach dispersion was replaced by an $\mathrm{Al}_{2} \mathrm{O}_{3}$ model provided by J. A. Woollam. We note oxidation is performed in our load lock, and not in the nitridation chamber. While the growth process cannot be monitored in situ, the wafer can be measured before and after oxidation. Each sample consisted of a $165 \mathrm{~nm}$ M1 Nb layer, covered with a 5-6 nm Al overlayer. The Al overlayer was then exposed to a $30,000 \mathrm{mT}$-s dose of $\mathrm{O}_{2}$ for oxidation. Thirteen samples were produced over the course of a year and applied to 4 different ellipsometry models. Model 1 is the SE extension of the SWE model and consists of a an Al-oxide film on an single $\mathrm{Al}$ substrate. Model 2 is the $\mathrm{Nb} / \mathrm{Al} / \mathrm{Al}-$ oxide model developed in part section 6.4 , but the unmodified 'textbook' models for $\mathrm{Nb}$ and $\mathrm{Al}$ provided by CompleteEASE® are used to create an off-the-shelf model as a baseline. Model 3 is an extension of Model 2, replacing the stock $\mathrm{Nb}$ dispersion 


\begin{tabular}{|c|c|c|c|c|c|c|c|}
\hline Model & $\mathrm{t}_{\mathrm{Al}_{2} \mathrm{O}_{3}}(\AA)$ & $\sigma_{t}(\AA)$ & $\sigma_{t}(\%)$ & $t_{A l}^{0}(\AA)$ & $t_{A l}^{\prime}(\AA)$ & $\frac{t_{A l_{2} \mathrm{O}_{3}}}{\Delta t_{A l}}$ & MSE \\
\hline \hline Model 1 & 10.42 & 0.67 & 6.4 & NA & NA & NA & 11.1 \\
\hline Model 2 & 4.92 & 2.62 & 53 & NA & NA & NA & 13.79 \\
\hline Model 3 & 9.81 & 0.98 & 10.0 & NA & NA & NA & 2.08 \\
\hline Model 4 & 9.78 & 0.39 & 4.0 & 63.9 & 56.1 & 1.23 & 1.95 \\
\hline
\end{tabular}

Table 5.1: Results of the four models averaged across 13 samples produced over the course of a year.

with our run to run recorded data, and the Al dispersion by our Lorentz-Urbach model, however, none of the self-calibration steps to correct for angle offsets and $\mathrm{Al}$ parameters are used. 'Model 4' is the complete model and process flow described in section 6.4 and shown in Figure 5.13. The offset angle is corrected, and our tabulated $\mathrm{Nb}$ and Lorentz-Urbach $\mathrm{Al}$ models are used.

We note that an additional benefit of being able to determine an $\mathrm{Al}$ overlayer thickness run to run, is that we can adjust deposition conditions as the target erodes to maintain a constant 5-6 nm Al overlayer thickness. A first order check to the validity of the determined $\mathrm{Al}$ thicknesses is to calculate the ratio of the thickness of the $\mathrm{Al}$ oxide film to the amount of $\mathrm{Al}$ consumed. For ideal hexagonal $\mathrm{Al}_{2} \mathrm{O}_{3}$ grown on ideal $\mathrm{BCC} \mathrm{Al}$, this ratio is 1.18 , which is within reasonable agreement with Model 4's determined value of 1.23 .

The average Al-oxide thickness, its standard deviation, and the MSE are tabulated in Table 5.1. While the MSE describes how well our model fits to our recorded data, and can give some incite to how well our model represents the physical process of AlN on thin $\mathrm{Al}$ overlayers, we note the precision of a model, or how repeatable its results are, is captured by the standard deviation. Because we are mostly concerned with run to run repeatability in order to produce trilayer of consistent $\mathrm{R}_{N} \mathrm{~A}$ values, standard deviation (precision) is the most important figure of merit for our application.

Model 1 (single Al-oxide film on an Al substrate) demonstrates the second best consistency in terms of standard deviation, despite the rather relatively large MSE. 
Models 2-4 take into account the thin $\mathrm{Al}$ overlayer ( $\mathrm{Al}$-oxide on $\mathrm{Al}$ on $\mathrm{Nb}$ substrates), however, they differ in the dispersions used for the layers, and whether or not a calibration of the angle offset from the initial $\mathrm{Si} / \mathrm{SiO}_{2}$ scan is performed. Model 2 uses the off-the-shelf models for the $\mathrm{Nb}, \mathrm{Al}$, and $\mathrm{Al}$ oxide films, serving as a baseline to test improvements from both the use of the dispersion models developed in this work and the importance for correcting an angle offset. Model 3 replaces the $\mathrm{Al}$ and $\mathrm{Nb}$ films from Model 2 with our Lorentz-Drude dispersion for Al, and our run to run recorded optical constants of the M1 Nb film as previously described in this chapter. Model 4, builds on Model 3, using the process described in Figure Figure 5.13 on page 108 to take into account angle offsets.

Models 2 and 3 are provided to show the significance of making intelligent assumptions when creating a new ellipsometric model, by both using thin film dispersions rather than bulk material values, and taking into account offset angles through run to run calibrations. Model 2 used bulk material optical constants, and was not be expected to fare well in terms of MSE as idealized bulk values differ from that of thin films. In addition, we would expect a relatively poor run to run standard deviation as optical constants of the materials may change over the life of the target, and the angle of incidence, due to how the sample is mounted, may change run to run. For Model 3, we expect an improvement in MSE as more accurate dispersions are used instead of idealized bulk values; however, we still expect a relatively worse run to run precision, compared to Model 4, as changes in the angle of incidence are not taken into account. Model 4, takes into account material dispersions and calibration of the angle of incidence, has the best results in terms of both MSE, and run to run precision. Compared to Model 1, which I note also includes the corrections to the angle of incidence and uses the optical constants recorded from the $\mathrm{Al}$ overlayer instead of bulk values for a fair comparison, Model 4 is better in terms of both the run to run standard deviation and average MSE. We excepted the MSE to improve, as the 
model is based on a more physically accurate representation of AlN growth on thin $\mathrm{Al}$ overlayers, and expected an improvement in the overall run to run precision, as any changes in the $\mathrm{Al}$ overlayer thickness that may occur over the life of the target are taken into account. For these reasons, Model 4 provides arguably the best SE model of the four in terms of accuracy (MSE) and precision (standard deviation) and is chosen to monitor AlN growth in this work.

\subsubsection{In Situ monitoring of AlN growth}

The majority of AlN formation through nitridation of the $\mathrm{Al}$ overlayer occurs while the sample is resting on the ellipsometry stage, and AlN thickness can be monitored throughout the growth process. As previously mentioned, some nitridation occurs while the sample is lowered on to the nitridation stage, however this initial growth is captured because we first scan our Al surface before the ICP is ignited. To provide a qualitative comparison of the different models over the entire growth time, AlN thicknesses vs nitridiation time for three trilayer samples that were exposed to a 200 W, 2.5 A, $5.0 \mathrm{mT}$ nitrogen ICP are shown in Figure 5.14 (Model 2 was neglected as the resulting AlN growth curves were far off from the rest) - more than a factor of 2 . Model 4 provides the most consistent results, in agreement with the $\mathrm{Al}$ oxide results.

A series of AlN based trilayer was produced over a period of a year and used to fabricate SIS junctions. The $\mathrm{R}_{N} \mathrm{~A}$ values of the junctions vs the ellipsometrically determined AlN barrier thickness is plotted on a semi-log plot and the $\mathrm{R}_{N} \mathrm{~A}$ values for AlN trilayer produced using $200 \mathrm{~W}, 2.5$ A, $5.0 \mathrm{mT}$ ICP nitridation conditions are plotted vs nitridation time in a log-log scale in Figure 5.15. We note, additional data points are included in the $\mathrm{R}_{N} \mathrm{~A}$ by nitridation time plot, as additional data collected before the ellipsometry model was implemented were included. The red shaded area represents the bounds of a least squares fit to the mean values \pm one standard deviation, and the solid red line is a least squares fit to the mean. We 

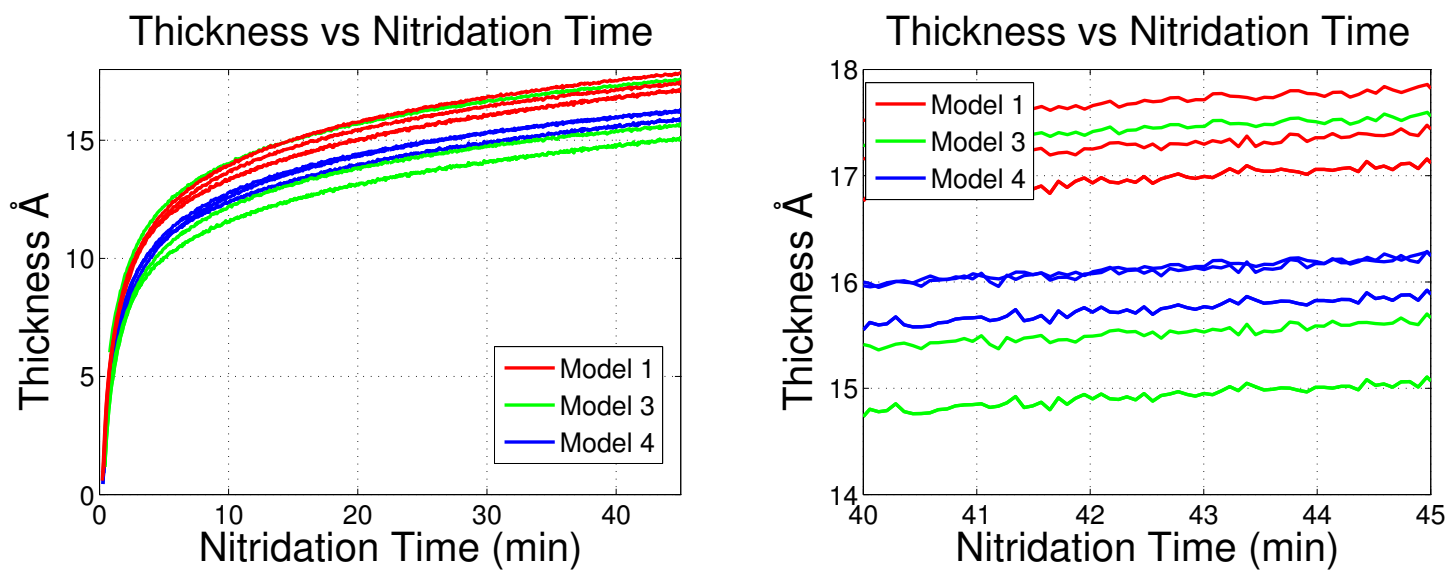

Figure 5.14: Left: AlN growth vs time in a $200 \mathrm{~W}, 2.5$ A, $5.0 \mathrm{mT}$ ICP. Right: Zoomed view of the final 5 minutes of growth showing the difference between the 3 models.

note for $\mathrm{R}_{N} \mathrm{~A}$ vs thickness, the data are best fit to $R_{N} A=\alpha e^{\beta T_{A l N}}$, and for $\mathrm{R}_{N} \mathrm{~A}$ vs nitridation time, the growth curves are approximately logarithmic in shape and the data are fit to $R_{N} A=\alpha e^{\beta \log (t)}=\alpha t^{\beta}$, where $\alpha, \beta$ are constants, $T_{A l N}$ is the AlN thickness, and $t$ is the nitridation time. Semi-log and log-log scales are used as these functions are linear in each respectively.

Noting the plot of $\mathrm{R}_{N} \mathrm{~A}$ with respect to ellipsometer thickness, error bars are shown as one standard deviation in $\mathrm{R}_{N} \mathrm{~A}$ and a $4 \%$ error for thickness as determined from the results in Table 5.1. For $\mathrm{R}_{N} \mathrm{~A}$ plotted with respect to nitridation time, the error bars for $\mathrm{R}_{N} \mathrm{~A}$ are one standard deviation of the data collected, however, there are no error bars in nitridation time as it is controlled using a timer. We note, there is a larger spread in the case in the $\mathrm{R}_{N} \mathrm{~A}$ vs $\mathrm{AlN}$ thickness plot compared to the $\mathrm{R}_{N} \mathrm{~A}$ vs time plot. We can attribute this, at least partially, to the $4 \%$ error in thickness, which due to its exponential influence, can correlate to a $\sim 25 \%$ error in $\mathrm{R}_{N} \mathrm{~A}$ for values in our range of interest, and combined with the standard deviation of our $\mathrm{R}_{N} \mathrm{~A}$ measurements, (typically 1-5\%), the uncertainty for predicting $\mathrm{R}_{N} \mathrm{~A}$ is greatly increased. However, when nitridation time is used to control $\mathrm{R}_{N} \mathrm{~A}$, we are not taking into account errors from our ellipsometric measurements, and the results fits reasonable well to $R_{N} A=\alpha t^{\beta}$, predicting a $\sim 10 \%$ error for $\mathrm{R}_{N} \mathrm{~A}$ values in our range 

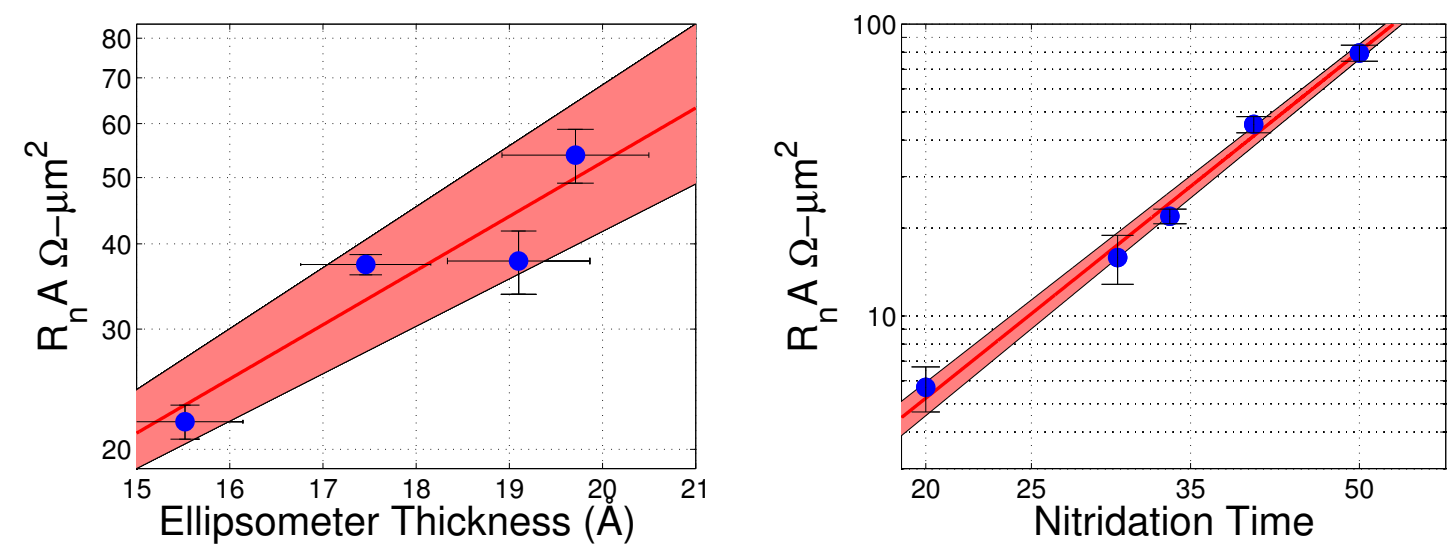

Figure 5.15: Left: Measured $\mathrm{R}_{n} \mathrm{~A}$ vs ellipsometrically determined AlN thickness using Model 4. Right: Measured $\mathrm{R}_{n} \mathrm{~A}$ vs nitridation time using a $200 \mathrm{~W}, 2.5 \mathrm{~A}, 5.0 \mathrm{mT}$ nitrogen ICP. We note there are more data points for nitridation time, this is because we had additional trilayer deposited before the ellipsometer model was put into effect, and included these points as well.

of interest.

\subsubsection{Conclusions}

The current density, and hence electrical properties, of SIS trilayer are exponentially dependent upon the tunnel barrier thickness. Precise control of the barrier thickness is integral in producing trilayer for SIS based electronics. While the oxidation of aluminum is well understood and a well behaved process which correlates to the pressure-time product of oxygen exposure during Al-oxide barrier growth, the nitridation of $\mathrm{Al}$ requires use of a nitrogen plasma, inherently a more complex process with additional variables than just pressure and time. As we investigate new nitridation conditions, growth rates may vary significantly and in situ monitoring of AlN growth could potentially allow us to produce trilayer with predictable $\mathrm{R}_{n} \mathrm{~A}$ values, limiting the number of test runs required while evaluating new conditions.

Our group first demonstrated the ability to qualitatively monitor AlN growth for SIS junctions in situ through the use of fixed angle SWE. However, fixed angle SWE was found to have drawbacks as it can only be used to analyze one free variable. 
As a result, it cannot correct for offset angles run to run (using our aforementioned calibration method utilizing the $\mathrm{Si} / \mathrm{SiO}_{2}$ substrate), and cannot be used to build thin film dispersions from the ground up, as this requires fitting both film thickness and dispersion parameters simultaneously. A SE model of AlN growth was developed as part of this work, with the ability to capture both the consumption of the $\mathrm{Al}$ underlayer as well as the AlN growth. We note capturing consumption of the $\mathrm{Al}$ underlayer has the additional benefit of allowing us to monitor its thickness over the life of our $\mathrm{Al}$ target and adjust conditions accordingly. When this model was applied to monitor the repeatable oxidation of $\mathrm{Al}$, the standard deviation in barrier thickness across 13 samples was $\sim 4 \%$. For AlN junctions, this would predict a spread of roughly $25 \%$ for $\mathrm{R}_{N} \mathrm{~A}$ values of $\sim 10-30 \Omega-\mu \mathrm{m}^{2}$, and a similar trend is observed in Figure 5.15.

Figure 5.15 shows the measured $\mathrm{R}_{n} \mathrm{~A}$ vs $\mathrm{AlN}$ thickness in a semi-log plot determined using model 4 , and an near exponential relationship betweem $\mathrm{R}_{n} \mathrm{~A}$ and thickness is observed. In Chapter 6, higher energy plasma nitridation conditions are investigated. Growth rates using the high energy conditions are about an order of magnitude higher than our standard $200 \mathrm{~W}$ ICP conditions, and we did not have a starting point in terms of nitridation time to produce trilayer with reasonable $\mathrm{R}_{n} \mathrm{~A}$. Using the ellipsometer to determine when to end nitridation, three samples were produced using three different uncharacterized nitridation conditions, and the standard deviation in $\mathrm{R}_{n} \mathrm{~A}$ was $25 \%$ of their average. We note, $\mathrm{R}_{n} \mathrm{~A}$ depends exponentially on barrier thickness, and a small error in thickness can correlate to a much larger error in $\mathrm{R}_{n} \mathrm{~A}$. This work, despite the advanced ellipsometric model developed, shows that the ellipsometer cannot be used run to run to 'lock in' on a specific $\mathrm{R}_{n} \mathrm{~A}$. However, when new nitridation conditions are investigated, trilayer can be produced close to the desired $\mathrm{R}_{n} \mathrm{~A}$, and the results can be plotted vs nitridation time to better predict the $\mathrm{R}_{n} \mathrm{~A}$ of future runs.

In addition to monitoring AlN growth across varying ICP conditions, we also 
note some additional benefits of the spectroscopic ellipsometer utilized throughout this work. Our research group has previously shown that in situ cleaning of the $\mathrm{Si} / \mathrm{SiO}_{2}$ substrate before $\mathrm{Nb}$ deposition significantly improves film quality, and in Chapter 3, we discussed the addition of an ion gun, for in situ cleaning, in our system [78]. SE allows us to monitor the amount of $\mathrm{SiO}_{2}$ etched by Ar ion milling, to both characterize the ion gun and develop a consistent cleaning procedure. Using the SE ellipsometric models developed in this chapter, we can also monitor the rate of background oxidation of our $\mathrm{Nb}$ and $\mathrm{Al}$ films, and, in conjunction with the RGA, this allows us to investigate the effects of various background impurities. We also note our group is beginning to investigate the use of reactively sputtered AlN to be deposited on top of the $\mathrm{Si} / \mathrm{SiO}_{2}$ substrate, to serve as an etch mask, and also as a potential directly deposited barrier layer for investigation of AlN trilayer with NbTiN base electrodes, which is not yet realized at the time of writing and discussed as part of an initial study in Appendix II. Using a Cauchy-Urbach dispersion model (5.19-5.20), both the refractive index and extinction coefficients of the AlN film can be monitored across various deposition conditions, and allow us to tailor our deposition conditions to best match the ideal values of stoichiometric AlN.

In conclusion, the use of a SE ellipsometer and our developed models, in conjunction with the rapid Al Quadlevel fabrication process and Test Mask set developed as part of this work, lays the groundwork to quickly investigate different SIS trilayer growth conditions. For future work to further improve upon this model, we note that surface roughness may have an effect on generating errors in measured barrier thickness. Surface roughness was not taken into account as part of our model, as it requires the use of an AFM to investigate surface morphology and one is not readily available in our research laboratory. Through the use of an AFM, roughness could be taken into account by adding an additional layer to our model using a effective medium approximation for the optical constants. 


\section{Chapter 6}

\section{Spectroscopic Analysis of ICP}

\section{Nitridation}

\subsection{Introduction}

As we have mentioned throughout this work, AlN barriers provide a convenient alternative to aluminum oxide barriers for use in SIS tunnel junctions with Nb base electrodes, allowing devices to be scaled to higher current densities. Various methods of AlN barrier formation have been discussed in Chapter 3, but one of the most promising methods, first reported by our group in 2007, is the formation of AlN tunnel barriers grown by ICP nitridation of thin $\mathrm{Al}$ overlayers on $\mathrm{Nb}$ base electrodes [24].

Despite producing low-leakage SIS junctions with current densities over $30 \mathrm{kA} / \mathrm{cm}^{2}$, a limitation of our original ICP configuration was a drift in run to run repeatability of $R_{N} A$ over time, presumably due in part to additional proprietary electro-mechanical circuitry contained in the ICP unit. In Chapter 3, we discussed how the ICP was reconfigured by CCR Technologies to remove the additional energy control circuitry in order to increase repeatability of $R_{n} A$ run to run. While this, along with the other 
discussed modifications to the deposition system, allowed us to realize AlN based SIS mixers; we note the IV characteristics of the AlN based Band-8 mixers, fabricated as part of this work in Chapter 3, contain excess subgap leakage current when compared to similar AlN based junctions previously fabricated at UVML [38]. However, the previously reported lower-leakage junctions were created using the originally configured ICP source that contained the aforementioned energy control circuitry, which is believed to affect AlN growth, possibly through changes in the relative species present in the plasma.

A correlation between junction quality and plasma dissociation has been reported for $\mathrm{Nb} / \mathrm{Al}-\mathrm{AlN} / \mathrm{Nb}$ junctions produced with an ECR plasma, but at the time of writing, nothing is reported for ICP [79]. It is argued that the relative nitrogen species generated by the modified ICP unit may differ from those of its original configuration. In this work, a quantitative measure of the relative dissociation of an ICP plasma is determined through the use of a commercially available Ocean Optics USB4000 Optical Spectrometer and an algorithm developed in this work implemented in MATLAB®. The effects of various processing parameters on the relative plasma dissociation of an ICP, and a correlation between plasma dissociation and quality of the resulting $\mathrm{Nb} / \mathrm{Al}-\mathrm{AlN} / \mathrm{Nb}$ junctions, are reported and discussed. The following references are used for entropy and enthalpy thermodynamic data used in free energy calculations throughout this chapter [80, 81, 82, 83].

\subsection{ICP Nitridation of AlN}

The simple oxidations of $\mathrm{Al}$ in an ambient background of $\mathrm{O}_{2}$ or water vapor:

$$
2 \mathrm{Al}+\frac{3}{2} \mathrm{O}_{2} \Longrightarrow \mathrm{Al}_{2} \mathrm{O}_{3}
$$




$$
2 \mathrm{Al}+3 \mathrm{H}_{2} \mathrm{O}(\mathrm{g}) \Longrightarrow \mathrm{Al}_{2} \mathrm{O}_{3}+3 \mathrm{H}_{2}
$$

have free energies of reaction, $\Delta G=\Delta H-T \Delta S,-1570 \mathrm{~kJ} / \mathrm{mol}$ and $-1370 \mathrm{~kJ} / \mathrm{mol}$ compared to the dissociate energy of $\mathrm{O}_{2}, 494 \mathrm{~kJ} / \mathrm{mol}$, and readily takes place at room temperature. A similar reaction for formation of AlN:

$$
A l+\frac{1}{2} N_{2} \Longrightarrow A l N
$$

has a free energy of reaction $\Delta G=-283 \mathrm{~kJ} / \mathrm{mol}$, but a negative free energy only means a reaction is possible, not that it will happen spontaneously. We note that the triple bond of $\mathrm{N}_{2}(942 \mathrm{~kJ} / \mathrm{mol})$ is stronger than the double bond of $\mathrm{O}_{2}(494 \mathrm{~kJ} / \mathrm{mol})$, and reactions of the type (6.3) do not happen spontaneously unless a mechanism to dissociate the $\mathrm{N}_{2}$ molecule is provided, so that the room temperature formation of AlN in $\mathrm{N}_{2}$ backgrounds is not observed. The formation of AlN by atomic nitrogen:

$$
N+A l \Longrightarrow A l N
$$

has a free energy of reaction $-708 \mathrm{~kJ} / \mathrm{mol}$. While these are all ideal first order approximations, it argues that either atomic $\mathrm{N}$, or highly energetic $\mathrm{N}_{2}$ molecules need to be present for the formation of AlN from an Al film. For these reasons, AlN barriers are often grown through plasma nitridation, and we will keep our focus on plasma nitridation using a helical ICP. A plasma is a partially ionized gas sustained through impact ionization between accelerated electrons and the relatively massive neutral molecules. Impacts with accelerated electrons can generate several species, ionized atomic and ionized diatomic nitrogen, $\mathrm{N}^{+}$and $\mathrm{N}_{2}^{+}$, as well as non-ionized but energetic atomic and excited diatomic nitrogen, $\mathrm{N}$ and $\mathrm{N}_{2}$. All of these species emit photons as they transition from higher to lower energy states. However, we note that the relative density of ionized species is generally a few orders of magnitude less than 


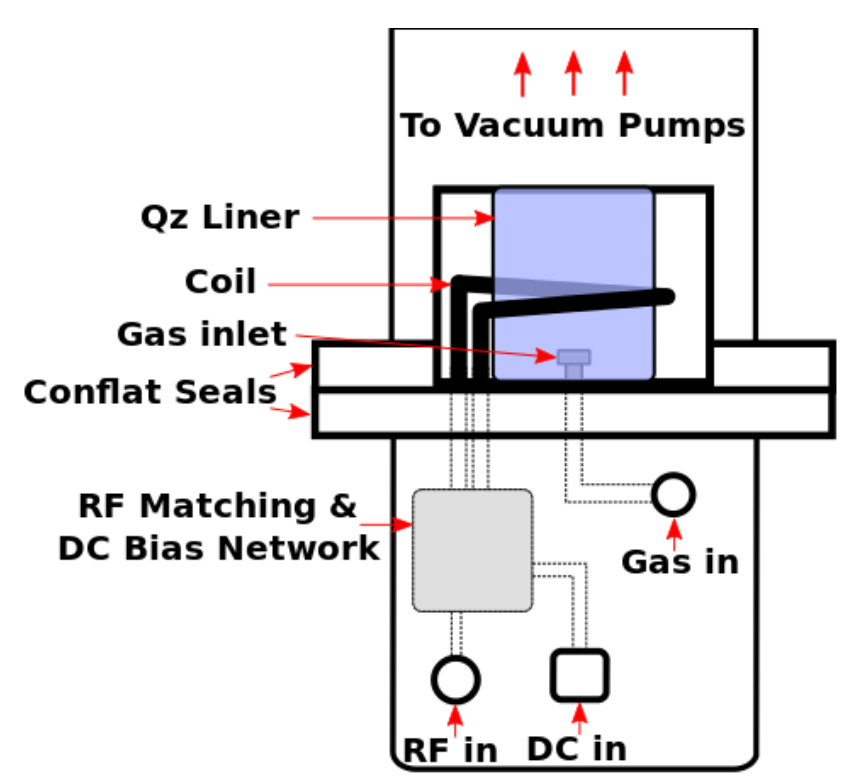

Figure 6.1: Cross sectional sketch of a helical ICP unit. A conducting coil is wrapped around a quartz liner inside a vacuum chamber, biased by both RF and DC currents it provides an alternating magnetic field inside the liner. Gas is fed into the liner through a gas inlet, and plasma is generated through induction. For AlN growth, nitrogen gas is used and the sample is positioned above the ICP unit as shown in Figure 5.5 on page 92 .

that of neutral species, and neutral species are responsible for the majority of the characteristic glow of a nitrogen plasma $[52,84]$.

A helical ICP consists of one or more turns of a metal coil, formed around a glass liner into which a gas is fed under vacuum, as shown in Figure 6.1. An RF bias is fed into the coil, and noting $\nabla \times \vec{E}=-\frac{\delta \vec{B}}{\delta t}$, energy is inductively coupled to the plasma through acceleration of free electrons by a circular E-field generated by the alternating B-field produced by the coil. In addition to the RF bias, it is common to feed a DC current to the coil generating a static component to the magnetic field, accelerating free electrons by means of the Lorentz force, $\vec{F}=q \vec{v} \times \vec{B}$, coupling additional energy.

The degree of dissociation of a nitrogen plasma is defined as the ratio of the amount of dissociated atomic nitrogen atoms to diatomic nitrogen molecules. Noting (6.4), it is clear that: (a) stoichiometric AlN growth can occur in the presence of neutral atomic $\mathrm{N}$, (b) N should be present in a nitrogen plasma through impact dissociation, 
and (c) the amount of impact dissociation, which forms atomic N, should be dependent on power coupled to the plasma. Given that the relative amount of atomic N in a plasma can be determined by a number of in situ instrumentation methods, and that atomic $\mathrm{N}$ should drive AlN growth, we decided to examine whether a correlation exists, in our current system configuration, between the relative dissociation of the ICP and the rate of stoichiometric AlN growth as well as the quality of the AlN tunnel barriers.

A direct method of measuring the amount of atomic $\mathrm{N}$ present is through use of a quadrupole mass spectrometer, however, the RGA in our system (which operates by means of a quadrupole mass spectrometer) is located far away from the ICP source, and the ionizing filament is not designed to operate at the several millitorr operating pressures used for AlN growth. An alternate means of determining a relative dissociation ratio is through observation of the spectral emissions from both the excited $\mathrm{N}$ and $\mathrm{N}_{2}$ molecules noting:

$$
\begin{gathered}
I_{N}=\alpha\left(\sigma_{e x 1} v_{e}\right) n_{N} n_{e} \\
I_{N_{2}}=\beta\left(\sigma_{e x 2} v_{e}\right) n_{N_{2}} n_{e} \\
\frac{I_{N}}{I_{N_{2}}} \propto \frac{n_{N}}{n_{N_{2}}}
\end{gathered}
$$

where $\alpha$ and $\beta$ are optical to electrical conversion constants, $\left(\sigma_{e x 1 / 2} v_{e}\right)$ are excitation rate coefficients, $n_{N}, n_{N_{2}}$, and $n_{e}$ are the number of $\mathrm{N}, \mathrm{N}_{2}$, and free electrons respectively, and $I_{N / N_{2}}$ are the measured intensities of the spectral emissions from excited $\mathrm{N}$ and $\mathrm{N}_{2}$ respectively $[85,86,79]$. Through the use of optical spectroscopy, we observe the emissions of atomic and diatomic nitrogen species present in an ICP plasma across various operating conditions to determine a relative dissociation and 
correlate to AlN growth rates and the quality of AlN based SIS tunnel junctions.

\subsection{Experimental Setup}

Our trilayer deposition system was equipped with an Ocean Optics USB4000 Spectrometer (configured with a $1200 \mathrm{l} / \mathrm{mm}$ grating centered at $735 \mathrm{~nm}$ and $25 \mu \mathrm{m}$ entrance aperture, producing a spectral resolution of $0.40 \mathrm{~nm}$ ) to record optical emissions from the nitrogen plasma and was coupled to the ICP chamber through a 600 $\mu \mathrm{m}$ diameter optical fiber attached to a $1 "$ diameter collimating lens and quartz window. The modified Copra DN-160 ICP source, described in Chapter 3, along with an ENI ACG-6B 13.56 MHz RF generator, and Delta Elektronika ES 030-5 DC power supply was used to generate a plasma using $99.9999 \%$ purity $\mathrm{N}_{2}$ gas. To ignite the plasma, a high pressure spike was used to reduce the breakdown voltage of the gas and generate seed electrons; once the plasma was ignited, it was able to be sustained at lower pressures. To determine a relative amount of dissociated nitrogen in the plasma, we made the assumption that the observed plasma spectrum is a mainly a superposition of the diatomic $\mathrm{N}_{2}$ spectrum and discrete spectral lines associated with atomic N. For the spectrum of diatomic $\mathrm{N}_{2}$, we focus our attention to the well studied $B^{3} \Pi_{g}-A^{3} \Sigma_{u}$ first positive system, which is the most prominent diatomic band with high intensity spectra appearing from 478 to $2531 \mathrm{~nm}$ [87]. For the spectrum of atomic nitrogen, we focus our attention on the $3 p^{4} S^{0} \rightarrow 3 s^{4} P$ nitrogen atomic triplet, as it lies within the range of optical emission captured by our spectrometer, overlapping spectra from the $B^{3} \Pi_{g}-A^{3} \Sigma_{u}$ system, and is one of brightest emissions from atomic $\mathrm{N}$ in plasmas [86]. To extract the separate $\mathrm{N}$ and $\mathrm{N}_{2}$ spectra, a similar approach to the one used by Endo is implemented and described below [79].

The spectrometer was initially calibrated using the $750.387 \mathrm{~nm}$ Ar line of an Ocean Optics HG-1 Mercury Argon Calibration Source, attached to the end of the 

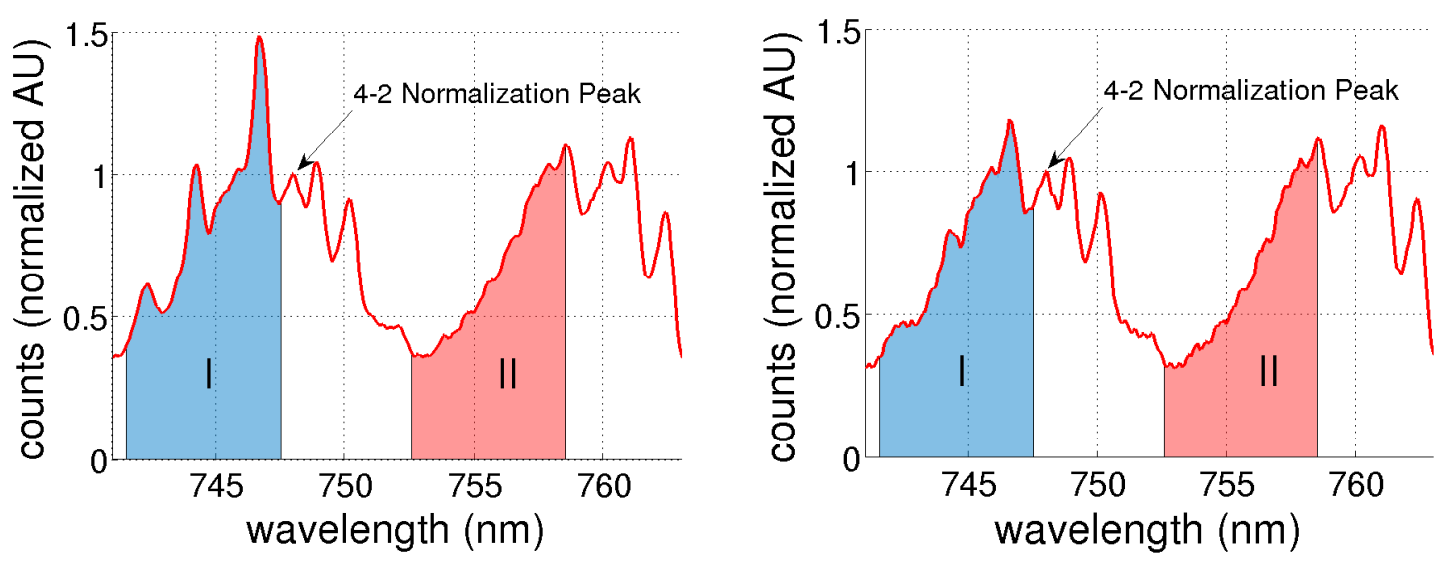

Figure 6.2: Spectrum (normalized to $747.9 \mathrm{~nm}$ ) of high and low power ICP at $5 \mathrm{mT}$ nitrogen. Left: High power $(600 \mathrm{~W}, 4.0 \mathrm{~A})$ plasma with prominent atomic $\mathrm{N}$ triplet at $742.36,744.23$, and $746.83 \mathrm{~nm}$. Right: Lower power $(450 \mathrm{~W}, 2.5 \mathrm{~A})$ plasma with reduced triplet peaks. Despite the difference between the two spectra in region I due to the varying atomic $\mathrm{N}$ triplet intensities, the general shape of region II remains unchanged.

$600 \mu \mathrm{m}$ diameter optical fiber which couples to the collimating lens. The nitrogen plasma spectrum from 741 to $763 \mathrm{~nm}$ was then recorded. Before starting the plasma, a reading of the dark chamber was recorded and the spectrum was saved and subtracted from the subsequent recorded spectrum of the plasma to remove any error from ambient light. We also note that the intensity of the spectra from various plasmas conditions vary, depending on the amount of applied ICP power. We also note the aforementioned calibration occurs at the end of the fiber optic cable before the collimating lens, and as such, doesn't calibrate the absolute intensity of the recorded spectra as any attenuation or gain from the lens is not taken into account. Additionally, our calibration standard produces discrete spectral peaks, and a proper calibration for absolute intensity requires the use of a well defined standard continuous over all wavelengths of interest (such as a black body source). However, the purpose of this initial calibration is to correct for error in recorded wavelength, which is not affected by the lens. To compensate for any drift in the instrument response function (determines how many 'counts' are reported for a given intensity at each 
wavelength) of our spectrometer over time, and to also allow comparison to other plasma conditions of varying intensity, the spectra are normalized to the spectral line of the $B^{3} \Pi_{g}-A^{3} \Sigma_{u}$ system at $747.9 \mathrm{~nm}$, unless otherwise noted. This spectral line was chosen as it is the first occurring spectral line of $\mathrm{N}_{2}$ outside of the $\mathrm{N}$ triplet region and prominently observed in all recorded spectra [87]. Normalization is performed by dividing the intensity of the spectrum at every wavelength by the intensity recorded by the spectrometer at $747.9 \mathrm{~nm}$, as shown in the code provided in Appendix V.

Figure 6.2 shows an ICP spectrum of a high power $(600 \mathrm{~W}, 4.0 \mathrm{~A}, 5.0 \mathrm{mT})$ and lower power $(450 \mathrm{~W}, 2.5 \mathrm{~A}, 5.0 \mathrm{mT}) \mathrm{ICP}$ recorded using our test setup. We note the occurrence of an $\mathrm{N}$ triplet at $742.36,744.23$, and $746.83 \mathrm{~nm}$, as well as the $\mathrm{N}_{2}$ peak, used for normalization, at $476.83 \mathrm{~nm}$. Between the two plasma conditions, the peak height of the atomic $\mathrm{N}$ triplet changes as applied power is varied, yet the general shape of the rest of the spectrum remains about the same. We note that we are observing only a subset of the spectral emissions from $\mathrm{N}$ and $\mathrm{N}_{2}$, and using this data to compare the amount of dissociation between various ICP parameters. We define our figure of merit as the 'relative dissociation', and while it is correlated to the degree of dissociation of the plasma, it may differ in magnitude from the absolute ratio of $n_{N} / n_{N_{2}}$. We define the relative dissociation, $R D=I_{N} / I_{N_{2}}$, as follows: First, we integrate the spectrum from 741 to $747 \mathrm{~nm}$, shown pictorially as the blue shaded 'region I' in Figure 6.2, which contains both the $\mathrm{N}_{2}$ background and atomic $\mathrm{N}$ triplet - Noting Figure 6.2, the red shaded 'region II from 752 to 758 nm qualitatively looks the same as region I, but lacks any atomic $\mathrm{N}$ spectral content. It is presumed that we can, to first order, extract the integral of the atomic $\mathrm{N}$ spectrum by simply subtracting a scaled integral of region II from region I, such that the integral of the $\mathrm{N}$ contribution is defined as:

$$
I_{N}=\int \text { region } I-A \int \text { region } I I
$$


To determine the scaling factor $A$, we record the spectrum of the highest pressure and lowest power sustainable plasma, as it should have little to no nitrogen dissociation, and set $I(N)=0$, solving numerically for $A$. Once $A$ is determined, we can define our relative dissociation as:

$$
R D=\frac{I_{N}}{I_{N_{2}}}=\frac{\int \text { region } I-A \int \text { region } I I}{A \int \text { region } I I}
$$

We note, that although $A$ is determined at the lowest power plasma condition, we perform the integral of region II from the spectrum of the operating condition in question. This procedure was implemented in MATLAB®, using the code developed as part of this work in Appendix $\mathrm{V}$, and was used to observe how process parameters affect the relative dissociation. To test the validity of our assumptions, results of this algorithm applied a variety of ICP conditions of increasing applied power as shown in Figure 6.3. The normalized spectrum of region I is shown in blue for each operating condition, while the spectrum of the scaled region II (assumed to have a similar shape), is shown overlaid in red. We note that the scaled spectrum of region II lines up qualitatively well to region I between the triplet peaks for all 4 plasma conditions, qualitatively validating our assumptions. If we look at the difference of region I and II shown in green, it appears to extract the atomic $\mathrm{N}$ triplet, which steadily increases for increasing applied power, indicating increased dissociation. Noting this qualitative agreement with our assumptions, the quantitative results of the relative dissociation using (6.9) are calculated through our algorithm applied to the recorded spectra from a variety of ICP conditions, are provided in the next section.

\subsection{Results}

Using the aforementioned algorithm to determine the relative dissociation of an ICP, we investigated how various processing parameters affected the relative dissociation 

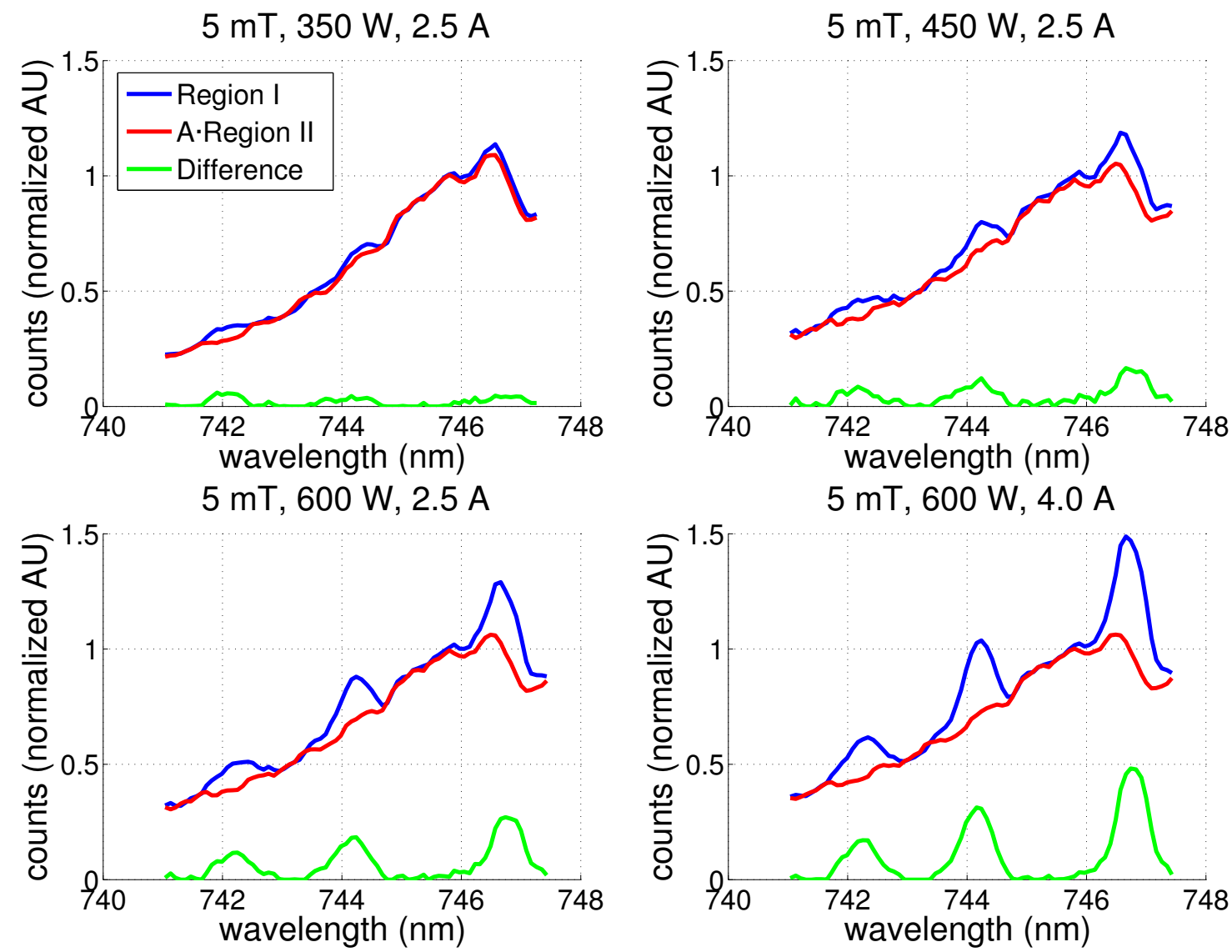

Figure 6.3: Results of our algorithm to extract the atomic N spectrum applied to a variety of ICP conditions of increasing power, from top left to bottom right. Both region I, containing spectral contributes from $\mathrm{N}$ and $\mathrm{N}_{2}$, and the scaled region II are overlaid. The difference of region I and the scaled region II, argued to extract the atomic $\mathrm{N}$ spectral contribution of region $\mathrm{I}$, is shown in green, and increases with increasing applied power. 


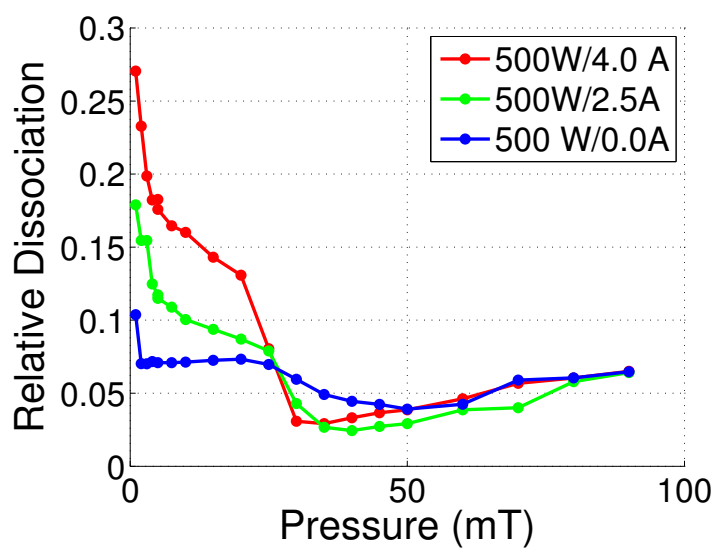

Figure 6.4: Relative dissociation with respect to operating pressure for 3 DC current levels is shown. A decrease in dissociation is observed for increased pressure for pressures below $\sim 25 \mathrm{mT}$ before a slight increase and leveling off.

of a nitrogen ICP. Building on this, we then investigated how the relative dissociation of the ICP correlates to both AlN growth rates and the quality of AlN junctions fabricated using trilayer grown with ICPs of varying RD values. The results are reported below.

\subsubsection{Effects of Processing Parameters on Dissociation}

The optical spectrum of our ICP was recorded from 741 to $763 \mathrm{~nm}$ across various operating conditions, and the relative dissociation was calculated using the algorithm described in section 7.3. The results of changes in relative dissociation with respect to changes in operating pressure, applied RF power, and DC current are presented below.

\subsubsection{Pressure}

The change in relative dissociation, with respect to changes in pressure, is shown in Figure 6.4 for $500 \mathrm{~W}$ applied RF power across 3 different DC current ranges. Below $\sim 25 \mathrm{mT}$, an increase in dissociation is observed with decreased pressure. For our helical ICP, both average ion energy and density decrease with increasing pressure, 


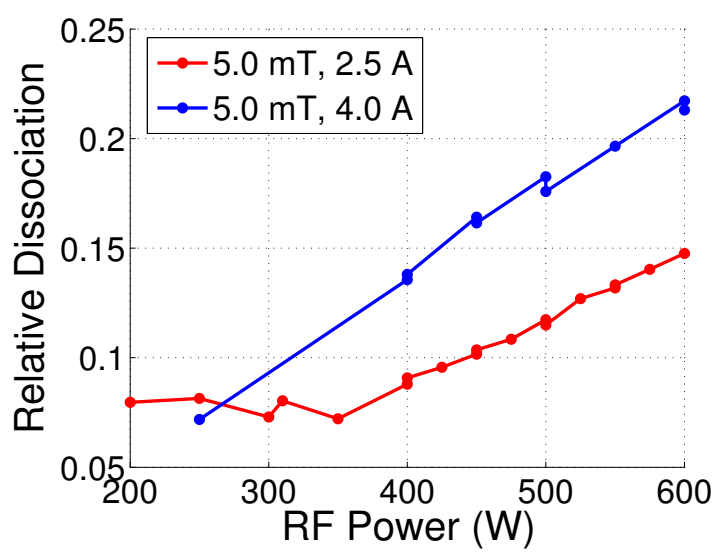

Figure 6.5: Relative dissociation of nitrogen plotted vs RF power for $2 \mathrm{DC}$ current levels. A near linear correlation between dissociation and current is show for our range of $\mathrm{RF}$ power.

decreasing rapidly until about 1-2 $\mathrm{mT}$ where they level off to a near linear relationship [88]. With lower energies and less electrons available for impact dissociation, a negative correlation between dissociation and pressure is expected at low pressures, and a rapid increase in dissociation for a decrease in pressure below $\sim 1 \mathrm{mT}$ as is observed. Past $25 \mathrm{mT}$, a slight positive correlation between pressure and dissociation is observed. We theorize that this is due increased impact ionization by reduced mean free path playing a larger role in dissociation as changes in average ion energy and ion current density level off. We note that our chosen ICP nitridation pressures are typically between 1-10 $\mathrm{mT}$ to both: (a) maintain a low average ion energy to prevent film damage (ion energy remains nearly constant for pressures $>\sim 1 \mathrm{mT}$ and independent of applied power for our ICP), and (b) to maintain a mean free path on the order of sample to ICP source separation [88]. We note that a near linear negative correlation between dissociation and pressure is observed in this region.

\subsubsection{RF Power}

The change in dissociation, with respect to applied RF power, is shown in Figure 6.5 for $5.0 \mathrm{mT}$ operating pressure across 2 different DC current ranges. A near linear 


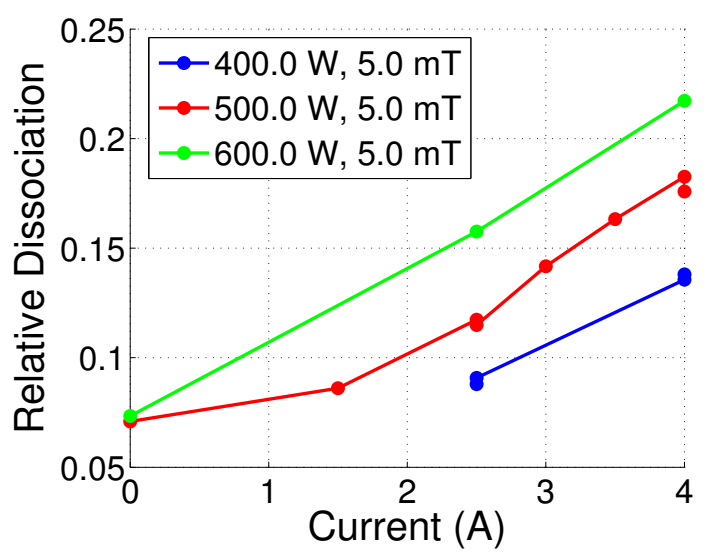

Figure 6.6: Relative dissociation of a nitrogen ICP plotted with respect to DC current. A near linear correlation between dissociation and current is shown for both 500 and $600 \mathrm{~W}$ of applied RF power.

relationship between dissociation and RF power is observed, which is consistent with the increased ion current density from increased RF power of an ICP. We note for $2.5 \mathrm{~A}$, a linear increase is not observed until $\sim 350 \mathrm{~W}$. While ICP sources ideally couple energy to the plasma through means of magnetic induction, H-mode, power is also coupled capacitively through the electrodes, E-mode, similarly to a parallel plate plasma source. With low applied RF power and DC current, ICP units may operate entirely in the E-mode regime, characterized by a visibly darker plasma where the onset of H-mode coupling occurs suddenly as power is increased [89]. For our particular system, a visible transition from E-mode to H-mode is observed for applied RF powers between $350-400 \mathrm{~W}$ for ICP at $5 \mathrm{mT}$ with a $\mathrm{DC}$ current of $2.5 \mathrm{~A}$. While we do not see an abrupt jump in dissociation between E-mode and H-mode plasmas, a near linear increase in dissociation with increased RF power is observed for H-mode operation.

\subsubsection{DC Current}

The change in dissociation with respect to changes in DC current is shown in Figure 6.6 for an operating pressure of $5.0 \mathrm{mT}$ across 3 different applied RF power levels. 
For $400 \mathrm{~W}$, the plasma was extremely dark below $2.5 \mathrm{~A}$, indicating operation in the H-mode, and was difficult to ignite and maintain. For these reasons, data was not collected for current values below $2.5 \mathrm{~A}$ for the $400 \mathrm{~W}$ plasma. We note an increase in dissociation with increase in applied current, similar to the case of applied RF power, is observed. This can be attributed to the increased energy coupled to the plasma and increased ion current density for high applied DC current.

\subsubsection{Effects of Dissociation on AlN Growth}

We have demonstrated how various processing parameters: DC current, RF power, and operating pressure, affect the relative dissociation of a nitrogen ICP. We note that it is conceivable that two plasma conditions can have a similar relative dissociation but different densities of $\mathrm{N}$ and $\mathrm{N}_{2}$ since we are measuring a ratio, $R D=$ $I_{N} / I_{N_{2}} \propto n_{N} / n_{N_{2}}$, and not absolute quantities. This is argued as variation in ion energies and mean free paths among various species with different scattering crosssections, might affect which species are excited. For ICP, where variation in average ion energy is nearly constant across the range of ICP parameters investigated, we'd expect any variation in excited species to be, at best, minimal. However, without the ability to perform a calibration for absolute intensity, we can't be $100 \%$ certain and this particular experiment was devised to reduce any potential ambiguity of the relationship between RD and $n_{N_{2}}$. For this experiment, we wish to observe how the density of the observed atomic $\mathrm{N}$ species might affect AlN growth rates, to test our hypothesis that atomic N may be one of the driving forces for AlN growth. For this particular experiment, we found ICP conditions with similar raw unnormalized $\mathrm{N}_{2}$ spectral intensities, and with varying unnormalized atomic $\mathrm{N}$ triplet intensity. Four unnormalized spectra collected using the same integration time, and collected consecutively within the span of an hour to minimize effects of any drift in the test setup, are shown in the right of Figure 6.7. The unnormalized $\mathrm{N}_{2}$ background intensity is 
nearly the same, but the atomic N triplet varies across the ICP conditions. Noting (6.5-6.6), we make the assumption that the the density of the excited $\mathrm{N}_{2}$ species we are observing is nearly constant among these four specific plasma conditions, but the density of excited atomic nitrogen we are observing for these four cases varies, and is also proportional to the relative dissociation. That is, $n_{N} \propto R D \cdot n_{N 2} \propto R D$, for an assumed constant $n_{N 2}$ across different conditions.

Over the course of a week, AlN films on $6 \mathrm{~nm}$ thick Al overlayers, deposited on optically thick $\mathrm{Nb}$, were grown using these four conditions via ICP nitridation, and the AlN growth was monitored via ellipsometry using the method described in Chapter 6. Due to the high growth rate at our highest dissociation condition, the nitridation was stopped at 30 seconds for that particular sample, to produce trilayer of a reasonable $R_{N} A$. While the samples grown at lower dissociation conditions received a longer nitridation time, the AlN film thickness was calculated after $30 \mathrm{~s}$ of growth for each sample for direct comparison. The resulting thicknesses (at $30 \mathrm{~s}$ nitridation time) vs the relative dissociation of the ICP is plotted on the left of Figure 6.7. We note a linear dependence on AlN thickness and relative dissociation (which, for these four cases at a minimum, is also proportional to the density of N), supporting our argument that the presence of the atomic $\mathrm{N}$ species plays a role in AlN growth.

\subsubsection{Effects of Dissociation on SIS Junction Quality}

We also investigated how junction quality correlates to the relative dissociation for AlN tunnel barriers formed by ICP nitridation. Three H-mode ICP conditions with varying degrees of relative dissociation, in addition to our standard E-mode $200 \mathrm{~W}, 2.5$ A, $5 \mathrm{mT}$ growth conditions, were chosen to create $\mathrm{Nb}(165 \mathrm{~nm}) / \mathrm{Al}-\mathrm{AlN}(6 \mathrm{~nm}) / \mathrm{Nb}(65$ $\mathrm{nm}$ ) trilayer on $330 \mu \mathrm{m}$ thick, $50 \mathrm{~mm}$ diameter DSP Si wafers with $300 \mathrm{~nm}$ of thermally grown $\mathrm{SiO}_{2}$. Noting the effects on AlN growth rates from the previous section, we picked 3 new H-mode ICP conditions for this particular test such that the relative 

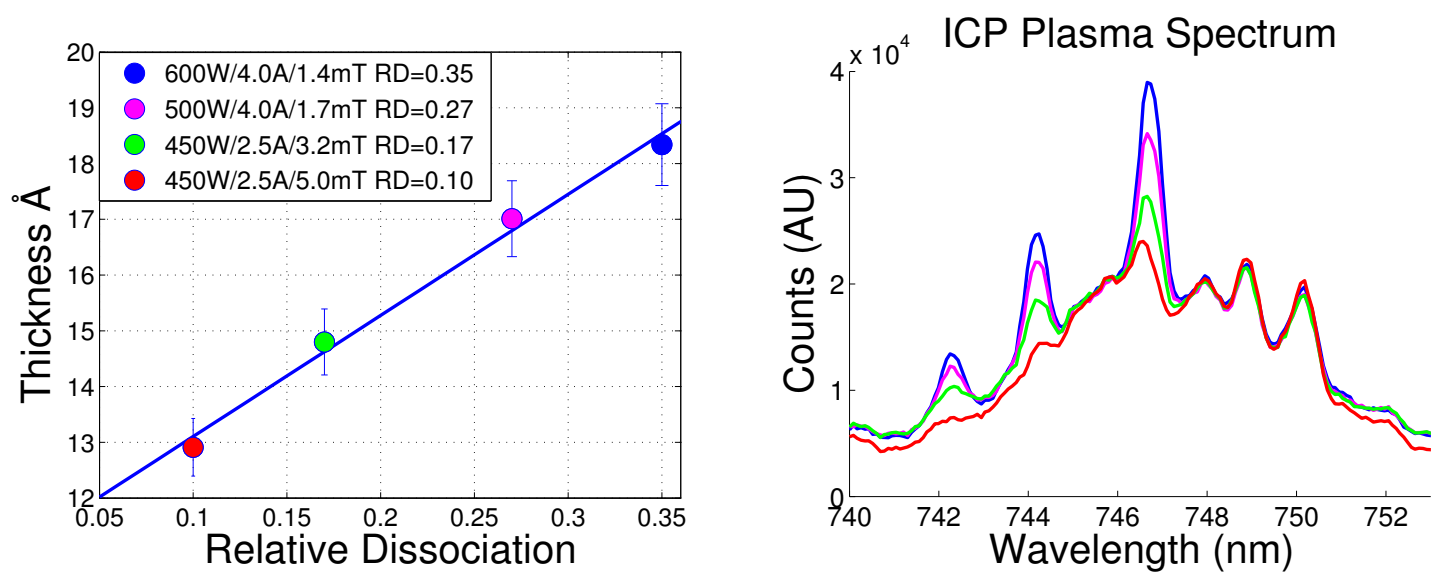

Figure 6.7: Left: AlN thickness at $30 \mathrm{~s}$ growth vs relative dissociation for 4 different plasma conditions as well as a linear fit to the data. Right: Unnormalized ICP plasma spectrum, each spectrum was captured using the same integration time and testing setup. Since the density of the observed atomic N species is proportional to RD for at least these four cases, it is therefore shown that growth rates are proportional to the density of the observed atomic $\mathrm{N}$ species.

dissociations were less than $\sim 0.25$ in order to obtain more modest AlN growth rates, and we also fixed the operating pressure to $5 \mathrm{mT}$ in order to reduce the number of variables that may affect AlN barrier quality. The normalized spectra of the 3 new H-mode ICP conditions chosen for AlN growth are shown in Figure 6.8. Additionally, one trilayer sample was prepared with AlN grown using the standard 'dark' E-mode conditions (200 W, 2.5 A 5.0 mT), used throughout Chapter 3. SIS test junctions, using the New Test Mask set and Al Quadlevel process described in Chapter 4, were fabricated and DC tested by means of submersion of the aforementioned POGO test chips in liquid helium. $\mathrm{R}_{S G} / \mathrm{R}_{N}$, as described in Chapter 4, was calculated for each series of test junctions, and is plotted vs the relative dissociation of the ICP plasma used for nitridation in Figure 6.8.

We note that there are two separate data points for the highest dissociation conditions, indicating two separate sets of samples. For these particular test samples, we aimed to produce trilayer with an $\mathrm{R}_{N} \mathrm{~A}$ value of $34 \mu \Omega / \mathrm{cm}^{2}$, the design spec for a current project we were working on at the time. Since these are uncharacterized ICP 

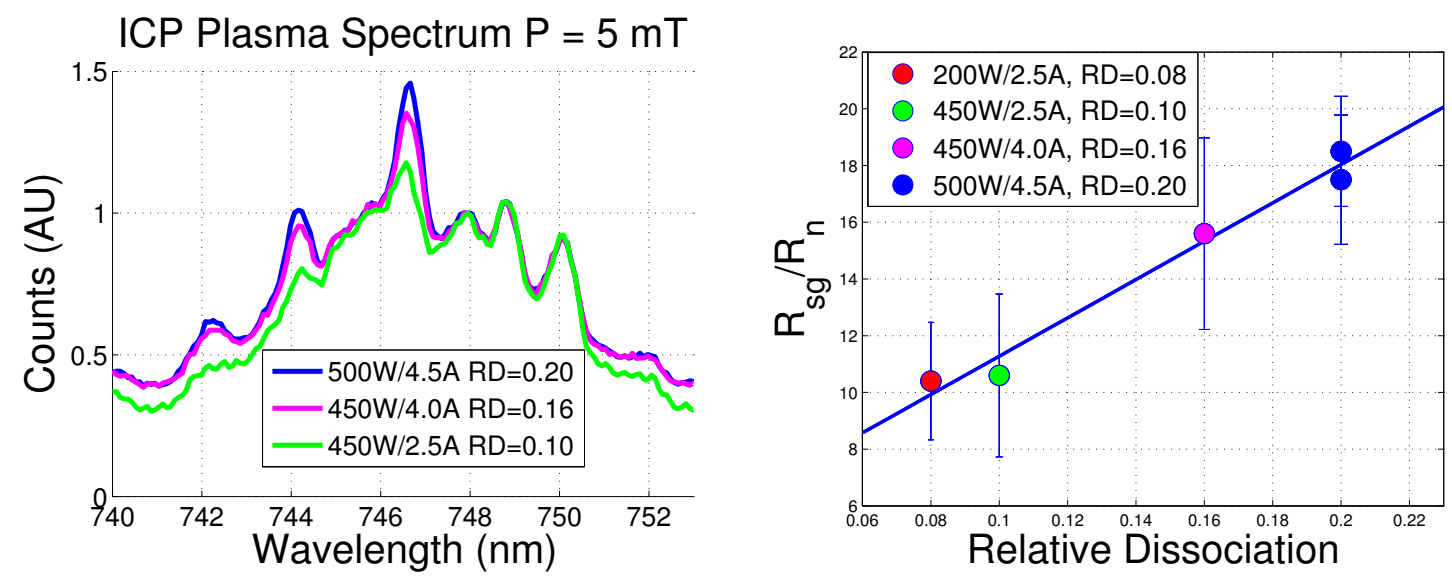

Figure 6.8: Left: Normal spectrum from the 3 ICP conditions used. Right: Average $\mathrm{R}_{s g} / \mathrm{R}_{n}$ of SIS junctions made across varying ICP conditions plotted against the relative dissociation of the ICP.

growth conditions, in terms of $R_{N} A$ vs nitridation time, we used in situ ellipsometry, described in Chapter 5, to determine when to end nitridation, bringing us within a factor of 2 of our desired value. In order to find a growth time that produces an $R_{N} A$ closer to our desired value, an additional trilayer sample was produced using the highest relative dissociation conditions of the set of four. The resulting $R_{N} A$ was $37 \pm 1.3 \mu \Omega / \mathrm{cm}^{2}$, and the resulting quality factor of the junctions was within a standard deviation of our previous trilayer produced using the same ICP conditions. Noting Figure 6.7 and Figure 6.8, a positive correlation between both AlN growth rate and junction quality to the relative dissociation is observed, and discussed in the following section.

\subsection{Summary and Conclusions}

At the beginning of this chapter, we showed that stoichiometric AlN growth can occur in the presence of atomic $\mathrm{N}$, and noting that dissociated atomic nitrogen species are present in a nitrogen plasma, we hypothesized that the growth rate of AlN grown by ICP nitridation of $\mathrm{Al}$ overlayers should correlate to the relative dissociation of the nitrogen ICP. We also noted that a correlation between nitrogen dissociation and the 
quality of AlN tunnel barriers grown by a nitrogen ECR plasma have been reported in the literature, and we conducted experiments to observe if a similar correlation between nitrogen ICP dissociation and junction quality exists for our particular system. In order to determine a quantitative figure of merit for relative nitrogen dissociation across varying ICP conditions, an optical spectroscopic method was used, focusing on the well studied $B^{3} \Pi_{g}-A^{3} \Sigma_{u}$ first positive system of $\mathrm{N}_{2}$ and the prominent $3 p^{4} S^{0} \rightarrow 3 s^{4} P$ atomic $\mathrm{N}$ triplet. Noting that the intensity of the spectral emissions from both $\mathrm{N}_{2}$ and $\mathrm{N}$ is proportional to their densities in the plasma, (6.5-6.6), we defined the relative dissociation as the ratio of their respective intensities, (6.9). An algorithm was described and implemented to extract contributions from both excited $\mathrm{N}_{2}$ and $\mathrm{N}$ of the spectrum from 741 to $747 \mathrm{~nm}$. This approach produced consistent and reasonable results when applied to our ICP tool, and using this algorithm we investigated the effects of applied RF power, DC current, and operating pressure with respect to the relative dissociation.

To investigate how the relative dissociation correlates to the rate of AlN growth, four specific ICP conditions with similar absolute unnormalized $\mathrm{N}_{2}$ spectral intensities, but with differing atomic $\mathrm{N}$ spectral intensities, were found and used for AlN growth. We argued that these four specific growth conditions, owing to their similar absolute unnormalized $\mathrm{N}_{2}$ spectral intensities, had similar $\mathrm{N}_{2}$ densities, but differed in the amount of atomic $\mathrm{N}$ available for AlN growth. A near-linear positive correlation between relative dissociation and AlN growth rate was observed. While we only observed the relative density of one particular species of non-ionized atomic N, and other species and processes may play a role in AlN growth, neutral species make up the majority of species in an ICP, and the evidence is compelling that atomic $\mathrm{N}$ plays a critical role in AlN growth. Furthermore, with this in mind, Figure 6.9 shows the difference of the spectrum recorded from our highest recorded dissociation ICP $(600 \mathrm{~W}, 4.0 \mathrm{~A}, 1.4 \mathrm{mT})$ and the spectrum recorded from of one of the lowest 
dissociation pure H-mode ICP (450 W, 2.5 A, $5.0 \mathrm{mT}$ ), both normalized to $747.9 \mathrm{~nm}$ and recorded from 580-890 $\mathrm{nm}$ (the maximum range of our Ocean Optics USB4000 spectrometer). To first order, the difference of the spectra should show which spectral features are increased as additional power is coupled to the plasma. Noting Figure 6.9 , the $3 p^{4} S^{0} \rightarrow 3 s^{4} P$ atomic $\mathrm{N}$ triplet used in our dissociation calculation is the most prominent series of peaks observed, and only the small peak at $648.3 \mathrm{~nm}$ can potentially be attributed to an ion species, $\mathrm{N}^{+}$. However, atomic $\mathrm{N}$ can also produce peaks of similar intensity, from $648.2-648.4 \mathrm{~nm}$, and noting our spectrometer's spectral resolution of $\sim 0.40 \mathrm{~nm}$, higher resolution is required to determine the true source of this peak. We do note however, below $400 \mathrm{~nm}$, a nitrogen plasma typically generates strong spectral lines for both $\mathrm{N}^{+}$and $\mathrm{N}_{2}^{+}$, and no contribution from the dominate $B^{3} \Pi_{g}-A^{3} \Sigma_{u}$ first positive system of $\mathrm{N}_{2}$, which may drown out ionic peaks in the visible spectrum $[90,87]$. We note that Ocean Optics, the manufacture of the spectrometer used in this study, offers alternate configurations which allow spectroscopic observation down to $165 \mathrm{~nm}$, and opens the possibility for future studies of ionic nitrogen species present in nitrogen ICP across various operating conditions [91].

It is interesting to note that our research group originally chose to investigate AlN barriers grown via ICP nitridation of $\mathrm{Al}$ overlayers, in part, because it potentially offers lower energies compared to parallel plate electrode generated plasmas. The expectation was that the lower energies associated with ICP would reduce damage caused by physical bombardment of the AlN barrier, and hence improve electrical characteristics. Indeed, SIS junctions made with ICP grown AlN tunnel barriers, at the correct conditions, are vastly superior to those grown by parallel plate nitridation methods. While it is clear that ionic species can have a significant effect on on AlN barrier quality, if such an effect exists for ICP grown AlN barriers, (where the ion energy is purposely kept relatively low by an appropriate choice of operating pressure), 
it is likely to play a secondary role to neutral atom $\mathrm{N}$ species.

To investigate how the relative dissociation correlates to junction quality, 4 growth conditions were chosen with varying levels of dissociation, one being our standard low power growth conditions. The pressure was fixed to $5 \mathrm{mT}$, to reduce the number of free variables and removed any possible pressure related effects, and dissociation was varied through change in Applied RF power and DC current. AlN based trilayer was made at all four growth conditions, and test junctions were fabricated and tested. The resulting $\mathrm{R}_{S G} / \mathrm{R}_{N}$ of the junctions was found to positively correlate with the relative dissociation of the ICP. We again note that we are only focusing on one of the many possible species which may affect AlN growth, but a clear correlation between junction quality and the relative dissociation of the plasma is observed. For our study on the effects of relative dissociation with respect to AlN growth rates, we argued that two plasma conditions $\boldsymbol{m i g h t}$ produce the same RD values but have different levels of atomic $\mathrm{N}$ present and, without a calibration for intensity, derived an experiment to reduce this possible ambiguity. However, for our ICP configuration and parameter space, it was argued RD will, in general, correlate to the amount of atomic N present. To support this argument, we plotted the in situ ellipsometrically determined AlN thickness at $3 \mathrm{~min}$ (the shortest growth time in our $R_{S G} / R_{N}$ study), to the relative dissociation of the ICP plasma for the $3 \mathrm{H}$-mode ICP conditions in Figure 6.10. A remarkably similar linear dependence on AlN thickness and RD is observed when compared to Figure 6.7 (our careful study of AlN thickness vs atomic $\mathrm{N}$ density, $n_{N}$, using RD calculated from raw unnormalized spectra recorded consecutively). The evidence is compelling that not only does the amount of atomic $\mathrm{N}$ present affect the rate of AlN growth, but it also affects the quality of the AlN film.

A similar trend relating junction quality to dissociation has been reported for $\mathrm{Nb}$ based SIS junctions with AlN tunnel barriers grown from ECR plasma nitridation of an $\mathrm{Al}$ overlayer, however, the results showed a much more dramatic effect [79]. They 


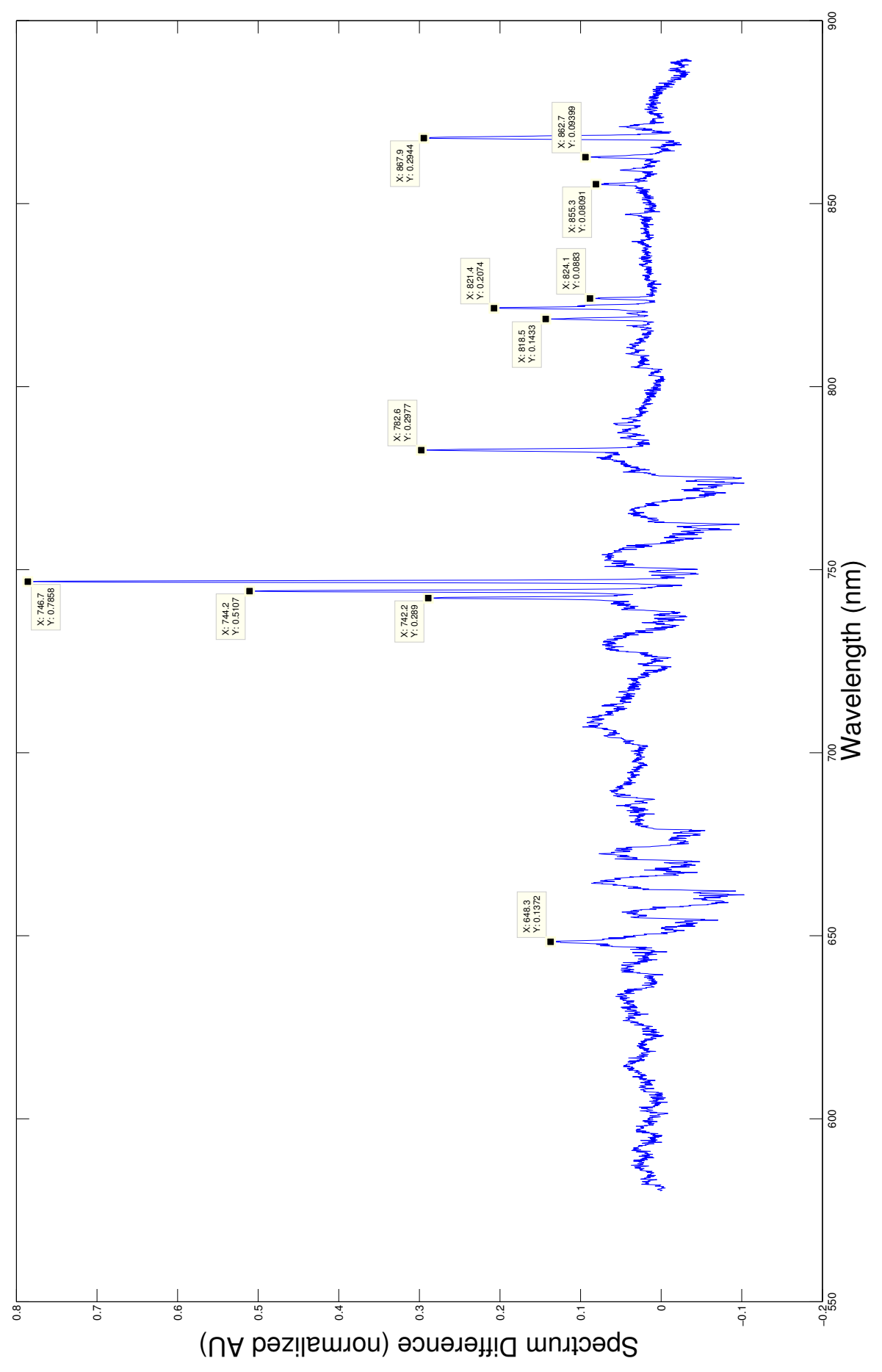

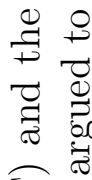

E.

घ.

ㄱ.

玄焉

$\circ$

$\forall \%$

而

尺릉

○

Oे

$\because 4$

.

需

ن

贾

욤

导

-

总

पू द्व

${ }_{00}^{2} \pm$

$\exists \exists$

ప

$\circ$

리 임

$0 . \approx$

$\because$ ठ

उ.

응

\& कै

官

$\exists$

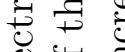

㟧.

क 0

क 0

ॠ

पै

○

ठ러

象㻤

$\ddot{\sigma}=$ की

○ $\Xi$

- $Ð$

$\exists$ ت

画题 


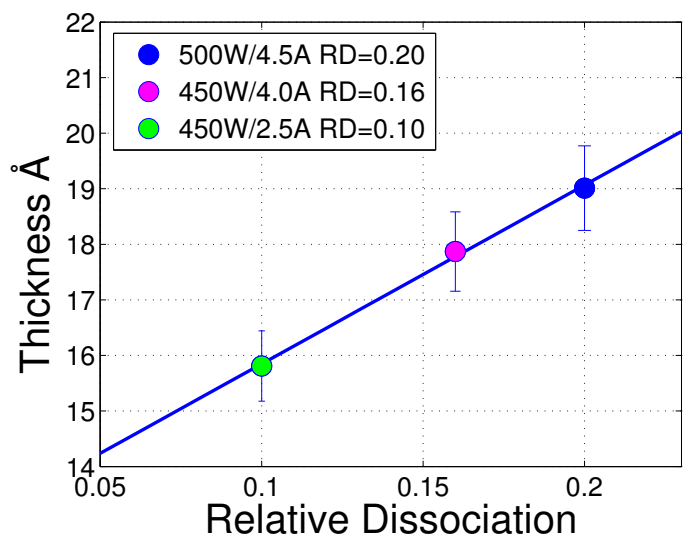

Figure 6.10: The AlN thickness (taken at 3 min nitridation time) is plotted with respect to the RD of the ICP used for AlN growth, using the $3 \mathrm{H}$-mode conditions presented in Section 7.4.3. A similar linear dependence between AlN thickness and $\mathrm{RD}$ is observed compared to the results presented in Section 7.4.2, arguing that the $\mathrm{RD}$ of our ICP, in general, correlates to the amount of atomic N present.

reported on two sets of SIS junctions, one grown at relatively low dissociation, and one grown at relatively high dissociation, and the resulting junctions produced $\mathrm{R}_{S G} / \mathrm{R}_{N}$ values of 1.3 and 16 respectively. Our low relative dissociation conditions routinely produce SIS junctions with $\mathrm{R}_{S G} / \mathrm{R}_{N}$ values in excess of 10 , while our higher relative dissociation ICP conditions produced a significant, albeit not order of magnitude, increase to $\sim 18$. With this disparity in mind, we note a few key differences between the work in [79] and the work presented in this chapter. The first difference is the working distance between the sample and plasma source. Our sample to source separation is $91 \mathrm{~mm}$, while for the work in [79], the separation is $142.5 \mathrm{~mm}$, and evidently through a considerably more constricted path. The second difference is that the base pressure in their work is stated to be $1.5 \mathrm{e}-7$ Torr, while the base pressure of the deposition chamber and nitridation chamber in our study ranged from 0.7-1.0e-8 Torr (partial pressure $\mathrm{H}_{2} \mathrm{O}$ 0.9-1.5e-9 Torr ) and 6.1-9.2e-8 Torr (partial pressure $\mathrm{H}_{2} \mathrm{O}$ 6.9-7.9e-9 Torr) respectively. While various partial pressures aren't reported in [79], the order of magnitude higher background pressure might exacerbate any effects that happen to be related to background oxidation. The third difference, is the plasma source 
and operating conditions used. They used an ECR plasma source, and in order to vary the dissociation of the plasma, the nitrogen flow rate was altered. The pressures for the low and high dissociation plasmas were $10 \mathrm{~Pa}$ and $<0.01 \mathrm{~Pa}$ respectively, corresponding to mean free paths of atomic $\mathrm{N}$ of $\sim 6 \mathrm{~mm}$ and $>500 \mathrm{~mm}$ respectively. We note, any atomic nitrogen present in the low dissociation plasma would undergo many collisions and have a higher chance of forming $\mathrm{N}_{2}$ before it can travel the 142.5 $\mathrm{mm}$ to reach the sample, exaggerating the effects of low dissociation. One the other hand, our operating pressure was fixed at $5 \mathrm{mT}(0.67 \mathrm{~Pa})$ across deposition conditions, as the dissociation of the ICP can be altered greatly through both applied RF power and DC current. The mean free path of atomic $\mathrm{N}$ at $5 \mathrm{mT}$ is $\sim 80 \mathrm{~mm}$, on the order of our source to wafer separation.

The overall conclusion from the work in this chapter is that a positive correlation exists between the relative ratio (6.9) of the spectral intensity of the $3 p^{4} S^{0} \rightarrow 3 s^{4} P$ atomic $\mathrm{N}$ triplet $(741$ to $747 \mathrm{~nm})$ to the spectral intensity of the $B^{3} \Pi_{g}-A^{3} \Sigma_{u}$ first positive system of $\mathrm{N}_{2}$ (752 to $758 \mathrm{~nm}$ ) in a ICP, and both the rate of AlN growth and the quality of SIS trilayer produced by ICP nitridation of thin Al overlayers on $\mathrm{Nb}$ electrodes for our particular setup. This provides convincing evidence that the presence of atomic $\mathrm{N}$ plays a critical role in the formation of high quality AlN tunnel barriers. We note that if the linear relationship between $\mathrm{R}_{S G} / \mathrm{R}_{N}$ and relative dissociation for our configuration shown in Figure (6.8) holds, it predicts the ability to create AlN junctions with average $\mathrm{R}_{S G} / \mathrm{R}_{N}$ values approaching 30 using the highest dissociation ICP conditions presented in Figure (6.7). We note that using higher dissociation ICPs presents a challenge as the AlN growth rate is significantly increased, leading to potentially difficulties in control of $R_{N} A$. However, we note that when Delft followed our research group's lead and began producing AlN trilayer through $\mathrm{H}$-mode ICP nitridation of $\mathrm{Al}$ overlayers using an identical ICP unit, they were able to reduce the nitridation rate by increasing the source to sample separation without a 
change in the spread of $R_{S G} / R_{N}$ [41]. For future work described in the next chapter, we plan to increase the source to sample separation through modification of our nitridation chamber, and investigate if a similar trend in $R_{S G} / R_{N}$ with respect to $\mathrm{RD}$ is observed.

These results present the question: why does increased atomic $\mathrm{N}$ content produce AlN barriers of higher quality? It is plausible that multiple species are competing to react with $\mathrm{Al}$, and atomic $\mathrm{N}$ may form a higher quality stoichiometric $\mathrm{AlN}$ barrier. Another possible explanation is that a high density of atomic N may inhibit the formation of conductive Al suboxides. All trilayer samples produced in this work were deposited with a partial pressure of water in the nitridation chamber from 6.9-7.9e-9 Torr, which is typical for 24 hours of load lock pumping time. Noting the background oxidation results presenting in Chapter 3, for partial pressures of water greater than 3e-9 Torr, oxidation is observed for timescales greater than roughly $3-5$ minutes. The nitridation times for a given $R_{N} A$ value are shorter for higher dissociation plasmas; for the trilayer samples produced in this chapter the times in order of increasing dissociation to produce an $R_{N} A$ of $\sim 35 \Omega-\mathrm{cm}^{2}$ are: $40 \mathrm{~m}, 12 \mathrm{~m} 30 \mathrm{~s}, 7 \mathrm{~m} 43 \mathrm{~s}$, and $3 \mathrm{~m}$ respectively. We note the following reaction:

$$
2 \mathrm{AlN}+3 \mathrm{H}_{2} \mathrm{O}(\mathrm{g}) \Longrightarrow \mathrm{Al}_{2} \mathrm{O}_{3}+\mathrm{N}_{2}+3 \mathrm{H}_{2}
$$

has a free energy of reaction of $-322 \mathrm{~kJ} / \mathrm{mol}$, and AlN films have been shown to readily oxidize in the literature in ambient environments [92]. We also note:

$$
2 \mathrm{~N}+\mathrm{Al}_{2} \mathrm{O}_{3} \Longrightarrow 2 \mathrm{AlN}+\frac{3}{2} \mathrm{O}_{2}
$$

has a free energy of reaction $+97 \mathrm{~kJ} / \mathrm{mol}$, and cannot happen spontaneously. While these are first order calculations, it argues that AlN should readily oxidize in the presence of water vapor, and even in the presence of atomic nitrogen, any formed 
oxides will remain. Given how junction quality correlates to relative dissociation and relative dissociation correlates to AlN growth rate, there is an ambiguity as to whether the quality is dependent solely on the growth rate or the relative dissociation of the ICP, or if these factors are all interrelated. Further data need be collected at various background partial pressures of water vapor and plasma dissociation to draw more conclusions, but we have laid the foundation in this work to measure partial pressures of contamination levels, record AlN growth in situ, and determine the relative dissociation of the nitrogen ICP used for AlN growth. Future proposed work and most recent results are discussed in further detail in the final chapter. 


\section{Chapter 7}

\section{Conclusions and Future Work}

Millimeter and sub-millimeter electronics seek to utilize one of the last uncharted regions of the electromagnetic spectrum, from roughly $100 \mathrm{GHz}$ to $3 \mathrm{THz}$. Despite the increased complexity of the often fusing together of optical and RF technologiesresearch in THz electronics has spawned interest in various imaging applications from medical research to atmospheric observations. We noted that one of the immediate benefactors of the ongoing research of $\mathrm{THz}$ electronics is the field of radio astronomy. A notable radio astronomy project is ALMA, a multi-national astronomical interferometer, of $667-12 \mathrm{~m}$ diameter radio telescopes designed to allow spans of $12 \mathrm{~km}$, located at 5,000 m elevation of the Chajnantor plateau in northern Chile. Inaugurated in March 2013, ALMA plans to observe our heavenly bodies in the information rich $30 \mathrm{GHz}$ to $950 \mathrm{GHz}$ spectrum (split into 10 individual frequency bands), and is the largest and most expensive ground based astronomical project even undertaken at a cost of $\sim \$ 1.4$ billion [93]. A project of such scale drives the significance of current research in the field of $\mathrm{THz}$ electronics.

Over the past few decades, superconducting circuits have found important applications in $\mathrm{THz}$ imaging. While many $\mathrm{THz}$ receiver technologies exist, the workhorse of many high-sensitivity THz frequency receivers is the SIS tunnel junction, as its 
performance is unmatched for frequencies up to $\sim 1 \mathrm{THz}$. Notably, Bands 3-10 of the ALMA project are based on SIS receiver technology. However, as receiver design frequencies approach and even exceed $1 \mathrm{THz}$, increased complexity in the realization of the SIS mixer circuits arises. At above $\sim 300 \mathrm{GHz}$, wavelengths enter the sub-millimeter regime and the corresponding receiver components continually shrink in size, increasing the difficulty of aligning and making electrical connections to devices. Ultra-thin few-micron-thick substrates are often required for mixer fabrication to both reduce RF losses in the substrate and increase ease of mounting into receiver setups. The realization of such ultra thin substrates required the development of a novel fabrication processes developed at UVML.

Additional constraints are also imposed on the materials used for realization of the SIS tunnel junctions and corresponding superconducting circuitry. As frequencies exceed the superconducting band gap of elemental $\mathrm{Nb}$, higher $T_{c}$ superconducting materials such as NbTiN are desired. However, we noted in Chapter 2 that the RF properties of $\mathrm{NbTiN}$ (due to their larger penetration depth) are different than that of $\mathrm{Nb}$ when incorporated in $\mathrm{RF}$ mixing circuits and, if the difference in penetration depth is not taken into account, errors of up to $50 \%$ in design parameters may result [25]. We also noted the prototypical $\mathrm{Nb} / \mathrm{Al}^{-} \mathrm{Al}_{2} \mathrm{O}_{3} / \mathrm{Nb}$ SIS junction, produced through the Gurvitch overlayer method, demonstrate excellent low-leakage IV curves for current densities below $\sim 8 \mathrm{kA} / \mathrm{cm}^{2}$ — for larger current densities, often required for higher frequency designs, the barriers are eventually shown to introduce excess leakage currents due to defects and pinholes [33].

A turning point in the development of higher current density SIS junctions was through the use of AlN as an alternative barrier material, notably through the ICP nitridation of $\mathrm{Al}$ overlayers first reported by our research group in 2007 [24]. While ICP grown AlN barriers have been shown to produce good quality SIS junctions with current densities in excess of $30 \mathrm{kA} / \mathrm{cm}^{2}$, a drift in $R_{N} A$ over time in our original ICP 
configuration made realization of AlN based SIS mixers with proper design impedance impossible at the time. Additionally, ICP nitridation of Al overlayers is inherently a more complex process than the simple oxidation of Al overlayers, and little is reported in the literature as to how and why various ICP conditions affect AlN growth. We also note that such studies ultimately require the fabrication and testing of multiple micron and even sub-micron SIS test junctions, a potentially time consuming process. My specific contributions to the field of both superconducting and $\mathrm{THz}$ electronics, with respect to this issues, are covered in the following sections as well as future proposed work building on what has been presented in this disseration.

\section{On-Wafer Penetration Depth Measurements of Su- perconducting Films}

When I first arrived at UVML, our research group had previously developed a process for the room temperature deposition of NbTiN films with $T_{c}$ in excess of $14 \mathrm{~K}$, and had demonstrated Nb/Al-AlN/NbTiN SIS junctions with good IV characteristics. However, the penetration depth of these films was unverified experimentally. As part of my previous thesis, my first contribution was the design of on-wafer test structures, and the corresponding mask set and fabrication processes that allowed the non-destructive on-wafer measurement of a films superconducting penetration depth using a vector measurements up to $50 \mathrm{GHz}$ [29]. This was accomplished through the use of a LakeShore CPX-1.5K cryostat and a pair of Picoprobe 50A-GSG-150 CPW probes, and the results were in excellent agreement with those predicted by theory.

We note that unlike previous penetration depth calculations by scalar measurements reported at the time, our measurements are calibrated vector measurements, allowing a reference plane to placed on a superconducting microstrip line. We note that not only can penetration depth be determined, but line loss can be observed 
through the calculated propagation constant of the transmission line. Currently our research group is investigating the possibility of extending the RF measurement range of our Lakeshore $1.5 \mathrm{~K}$ cryostat to frequencies up to $220 \mathrm{GHz}$. We note that our method of measuring penetration depth on-wafer can be scaled to higher frequencies through use of the parameterized HFSS models of our test devices, and may prove useful in future investigations.

\section{Realization AlN Mixers on Thin-Si substrates}

In Chapter 3, we presented the results of ALMA Band-8 mixers, based on Nb/AlAlN/Nb SIS junctions fabricated on $3 \mu \mathrm{m}$ thick Si substrates. This was the first time AlN based SIS mixers have been realized on thin-Si substrates, and demonstrates the importance of two specific contributions as part of my research at UVML.

\section{Backside Compensation of Thin Si Substrates}

Upon my arrival at UVML, our research group had previously realized HEB mixer circuits fabricated on thin-Si substrates. The initial implementation of thin-Si SOI process at UVML was initially designed for use with HEB mixers, successfully fabricated on a bare silicon substrate [20]. However, when this process was applied to create ultra-thin SIS mixer chips (which require a thermally grown oxide to provide an appropriate surface for deposition of the $\mathrm{Nb}$ based SIS trilayer), the initial chips fabricated at UVML exhibited pronounced curling when released from their carrier. The bending was a result of the thermal oxide film stress, and we note such curved chips were found unsuitable for mounting in split block waveguides. As mentioned in Chapter 2, my work involved the development of a stress compensation scheme to control wafer bowing by means of depositing a precisely characterized compensation film on the backside of the chip. This allowed our research group to successfully 
realize aluminum oxide based SIS mixers, fabricated on thin-Si substrates with Au beam leads, for the SuperCam project [94]. Despite this, there were still limitations to overcome for the realization of AlN based SIS mixers.

\section{Modifications to Trilayer Deposition System}

Upon the start of my $\mathrm{PhD}$ research, our research group had demonstrated the ability to produce fairly low-leakage high-quality Nb/Al-AlN/Nb SIS junctions utilizing ICP grown AlN barriers, however, it was shown that the repeatability of $R_{N} A$, drifted over time by up to an order of magnitude [38]. Such a drift in $R_{n} A$ made it impossible to realize SIS based mixers of proper design impedance. Several modifications to our trilayer deposition system were undertaken to increase repeatability of $R_{N} A$ including, (1) a reconfiguration of our ICP unit, (2) addition of 2 turbo pumps and gate valves to isolate the load lock during venting, (3) addition of an RGA in both the deposition and nitridation chambers to monitor background gas contamination levels, and (4) the addition of a dedicated ion gun to allow in situ cleaning of our samples away from critical components. The details of the modifications are covered in both Chapter 3 and Appendix III. The ultimate result of these modifications was a significant increase in the repeatability of $R_{N} A$. These contributes gave our research group the ability to reasonably predict an $R_{N} A$ value for a given nitridation time and, combined with the stress management scheme from my previous thesis, allowed the realization of AlN based SIS mixers on thin-Si substrates. We note that the resulting ALMA Band-8 frequency mixers had good SIS characteristics and met all ALMA Band-8 specifications. 


\section{Rapid Fabrication and Testing of Micron and Sub- micron SIS Junctions}

We noted in Chapter 3, that during the time we began modifications to our trilayer deposition tool, we lost the ability to create AlN trilayer with good IV characterized due to our ICP being incorrectly reconfigured by CCR Technologies. Before this became apparent, every aspect of fabrication was scrutinized over a year bring our research to a halt. The amount of fabrication undertaken during this period demonstrated the need for a more rapid fabrication and testing process, which still mimicked our current "pentalevel" process, to realize micron and sub-micron SIS junctions for testing purposes. This work consists of 3 specific contributions: (1) the development of a time efficient fabrication process, (2) the development of a new 'Test Mask' set and fabrication process, and (3) the conversion of our manual IV testing setup to digital data capture and analysis.

\section{Al Quadlevel Process}

Through the development of a self-aligned junction definition process using a quadlevel NFR/Poly/Al/NFR(image) stack, fabrication time was significantly reduced to as little as a week. Despite the trade-off between reduced fabrication time and control in junction size, a modest variance of $\sim 10 \%$ was observed for $1.7 \mu$ m junctions across a $50 \mathrm{~mm}$ wafer and predicts $\sim 15 \%$ variation for $1.0 \mu \mathrm{m}$ junctions. The reduced fabrication time has proven itself to be indispensable for relatively quick evaluation of new trilayer materials, and investigation of various processing parameters. For future work, we note a detailed analysis of the $\mathrm{Al}$ undercut across multiple junction sizes could allow for compensation by placing a positive bias on the junction mask to create slightly large mask features to account for reduction in size due to undercutting.

We have shown in Chapter 4 that the highest quality junctions produced from 

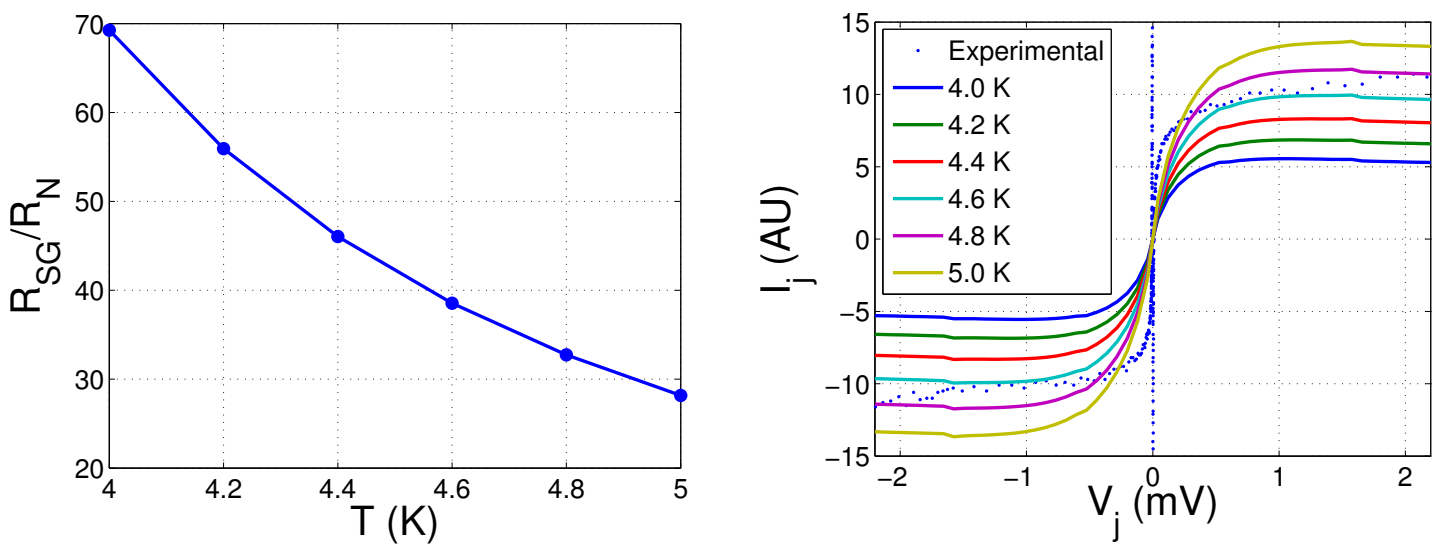

Figure 7.1: Left: The temperature dependence of $R_{S G} / R_{N}$ as predicted by the BCS density of states and Fermi-Dirac distribution. Right: The corresponding subgap leakage at various temperatures predicted by BCS theory overlaid on the subgap leakage of one of the highest quality junctions from Chapter 4.

the Al quadlevel process produced an $R_{S G} / R_{N}$ value approaching 30 . We noted that testing took place by submersion in liquid helium at $\sim 4.2 \mathrm{~K}$ - since the temperature was not absolute zero, some level of subgap leakage was expected due to the electron energy distribution. Using Equations (2.2-2.3) and assuming a Fermi-Dirac distribution for the quasi-particles, I generated synthetic SIS IV's at various temperatures to investigate the amount of subgap leakage. I note that I did not take into account the temperature dependence of $\Delta$ (as $\left.T \lesssim T_{c} / 2\right)$ or the energy dependence of $N_{n}(E)$ (as $\mathrm{E}$ is on the order of meV). Figure 7.1 shows the resulting ideal temperature dependence of $R_{S G} / R_{N}$ from 4 to $5 \mathrm{~K}$, as well as the corresponding ideal subgap leakages

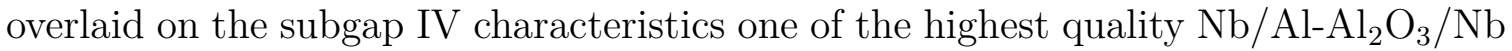
junctions fabricated using the $\mathrm{Al}$ quadlevel process. This result is worth noting as it sets a practical limit on $R_{S G} / R_{N}$, for dip testing in liquid helium, as we further refine our fabrication process and seek conditions to increase the quality of our ICP grown AlN tunnel barriers. 


\section{New Test Mask Set}

While developing the Al Quadlevel process, we note the current mask set, used to fabricate test junctions, required the entire wafer to remain whole during the process. As we varied process parameters, each iteration required a separate trilayer sample and we noted using multiple trilayer runs added some ambiguity to our results. A new test mask set was designed that allows the wafer to be scribed into four pieces along any part of the fabrication process. In addition, multiple test blocks were included to troubleshoot various failure mechanisms that were observed by our research group during the fabrication of mixer wafers. Chapter 4 describes how this mask set was used to successfully compare 4 different $\mathrm{SiO}_{2}$ deposition methods, and demonstrated the striking effect that variation of one processing parameter can have on junction quality. For direct comparison of various processing parameters, as well being able to save a quarter of known 'good' trilayer as a standard for future investigations, this new mask set will prove itself an extremely valuable tool.

\section{Digital IV Recording}

We also note, for this work, we made the transition from using a manual XY pen plotter to record IVs, to the use of a GRAPHTEC GL900 MIDI logger and custom software written in MATLAB®, the GUI is shown in Figure 7.2 and the details are provided in Appendix V. An immediate benefit was that, combined with the POGO dip testing technology, IVs could be recorded quicker (it is not uncommon to test 50 junctions in one day). Additionally, the use of software to both plot the resulting IV characteristics and calculate $R_{N} A$ and $R_{S G} / R_{N}$, both removes any user bias in their calculation, and allows for larges numbers of test junctions to be used for statistical analysis. For example, the $\mathrm{SiO}_{2}$ comparison in chapter 4 was the result of testing over 100 single and multi junction test elements, such a large number of IVs for

analysis is very time consuming and cumbersome using a manual pen-plotter and/or 


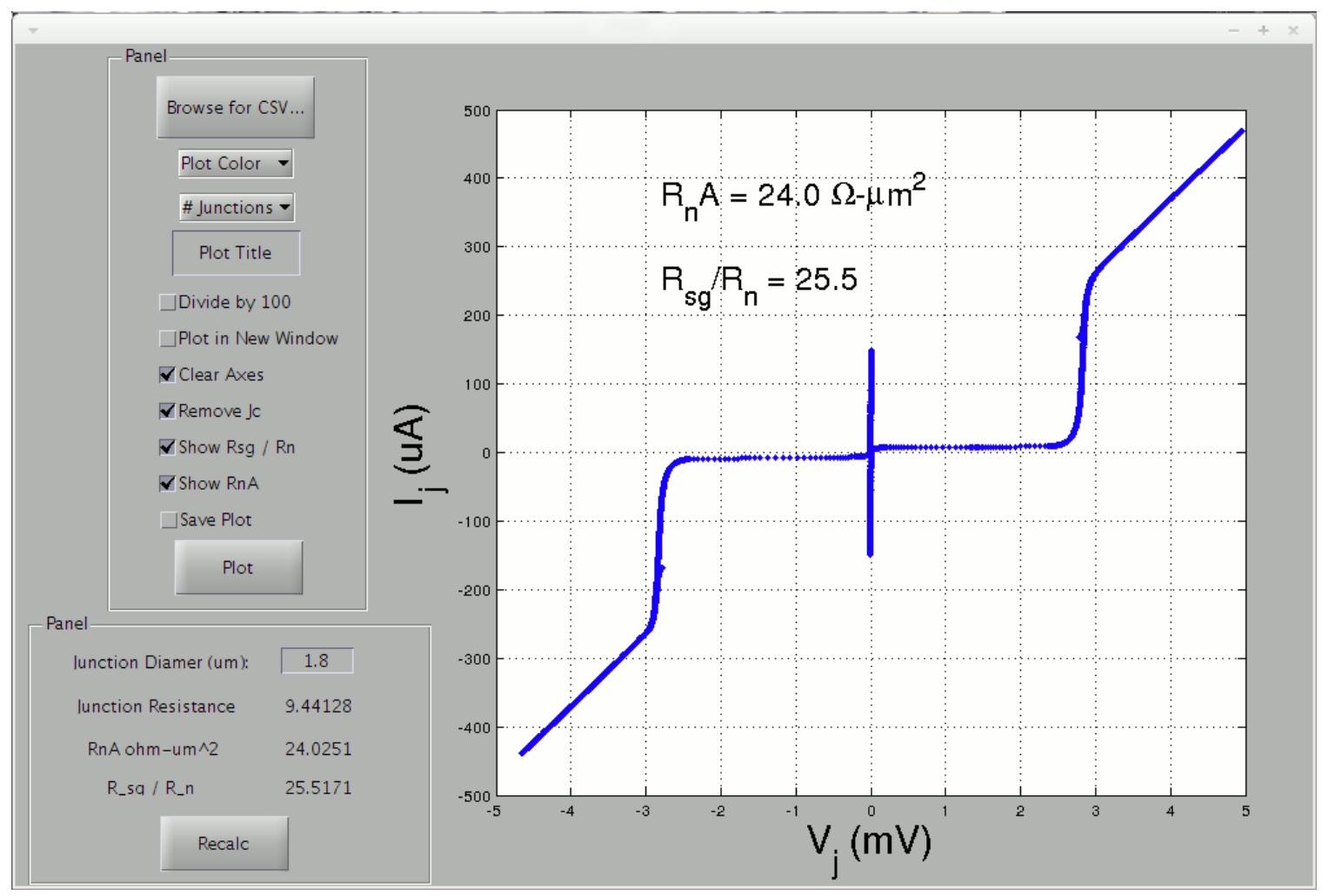

Figure 7.2: GUI of IV plotting software.

performing $R_{N} A-R_{S G} / R_{N}$ calculations by hand. For future work, there is always the possibility to port this program to an open source language such as Python, removing the licensing issues which periodically arise from MATLAB® such as the need for a Internet connection. I believe this program will continue to prove itself indispensable when analyzing large numbers of SIS test junctions for statistical analysis in future investigations.

\section{In Situ Monitoring of AlN Growth}

In Chapter 5, we discussed the development of an in situ method of monitoring AlN (or Al oxide) growth using of a J.A. Woollam spectroscopic ellipsometry and CompleteEASE® software package. My contribution in this work involved the ground-up development of an advanced self-calibrating ellipsometric model that provides an in 
situ quantitative measure, of both the increasing AlN film thickness as well as the decreasing Al overlayer thickness, during AlN growth on thin Al overlayers. This model was also used, in conjunction with RGA data, to monitor unwanted background oxidation, providing insight to the effects of various background partial pressures. Using this model to monitor the growth of 3 new uncharacterized nitridation conditions, all three samples had $R_{N} A$ values withing a factor of 2 of each other. For future applications, when new trilayer growth schemes are investigated, such as using higher dissociation ICP or direct deposition of AlN through reactive sputtering, trilayer can be produced close to the desired $R_{n} A$ for testing purposes. For future work, we note that there is room for improvement in the model by taking into account surface roughness through use of an AFM.

We also note, as we investigate the possibility of using directly sputtered AlN tunnel barriers, the use of in situ ellipsometry not only allows the measurement of AlN film thickness, but can also give insight to how various deposition conditions affect the optical properties of the AlN film. AlN films are formed by reactive sputtering of an $\mathrm{Al}$ target, a more complex process than the sputtering of a stoichiometric target by inert species, and finding optimal deposition conditions are likely to play a role in film quality. A future study could involve tailoring AlN deposition conditions such that the optical constants of the AlN films better match ideal bulk values.

Additionally, recording ellipsometric data for every trilayer run allows better monitoring of any drift in our deposition processes. In Chapter 5, we have shown that not only can the Al overlayer thickness be measured by the developed model, but resistivity of the Al film can also be calculated. This allows us to not only monitor changes in $\mathrm{Al}$ overlayer thickness over the life of the target (allowing us to adjust deposition conditions to maintain a constant $\mathrm{Al}$ overlayer thickness), but monitor resistivity run to run for any sudden changes that may be indicative of target contamination. We also noted that a correlation between $\mathrm{Nb}$ film stress and the measured index of refraction 
was observed. Nb film quality is maintained, over the life of a target, through periodically adjusting deposition conditions to maintain a constant slightly compressive film stress. The Nb optical constants are recorded for each run using the developed AlN growth model, and as such, provides yet another data point to monitor run to run.

\section{Spectroscopic Analysis of ICP}

As part of the work in this dissertation, an algorithm was developed to determine a quantitative measure of the relative dissociation of a nitrogen ICP using a commercially available spectroscopic ellipsometer. When implemented in MATLAB®) (code shown in Appendix V), the algorithm can be applied to arbitrarily large datasets, automatically parsing and performing the algorithm on all stored spectrum files, storing the ICP parameters (operating pressure, RF power, DC current) and their corresponding relative dissociation in an array for use in your plotting software of choice. After ICP conditions were chosen using these data, the relationship between relative dissociation of the ICP and various processing parameters was invested. When $\mathrm{Nb} / \mathrm{Al}-\mathrm{AlN} / \mathrm{Nb}$ trilayer was produced, using ICPs with various amounts of relative dissociation, a positive correlation between relative dissociation and both the AlN growth rate and the average quality (defined as $R_{S G} / R_{N}$ ) of the resulting junctions was observed

Unlike the oxidation of $\mathrm{Al}$ overlayers, the ICP nitridation of $\mathrm{Al}$ overlayer is inherently a more complex process that is not fully understood at the time. As such, these results are personally some of the most interesting to me and argue that atomic $\mathrm{N}$ plays a significant role in the formation of high quality AlN. Between our low and high dissociation conditions, a near factor of 2 increase in $R_{S G} / R_{N}$ was observed, and a factor of 3 increase is predicted for our highest dissociation conditions. However, 
we note that a drawback to the use of higher dissociation ICPs is that the increased deposition rates significantly reduce required nitridation times, and may affect the ability to reasonably control $R_{N} A$.

We note that by increasing the ICP to sample distance, we can likely reduce the AlN growth rate for higher dissociation ICPs. Noting this, a 5" long tube extension was designed (shown in Appendix III), and will sit between the bottom of the existing nitridation tube and ICP, approximately doubling the ICP to sample separation. The hope is that the increased separation will reduce the reduce the AlN growth rate, allowing the ability to control $R_{N} A$ at higher dissociation conditions. Another notable feature is that the tube extension will contain a 2" quartz view port onto which we can attach a collimating lens for spectroscopic. The lens will be in close proximity to the ICP, and is believed to better sample the emitted radiation for analysis.

For future studies on the effects of relative dissociation of junction quality, more IV data need be collected from trilayer produced from varying ICP conditions, as well as varying amounts of background gas impurity levels. While a positive correlation between relative dissociation and junction quality was observed in this work, it is still unknown why this trend was observed. One theory is that there are competing species present, all of which react with the $\mathrm{Al}$ overlayers. If the competing species are various oxygen containing molecules (such as $\mathrm{H}_{2} \mathrm{O}, \mathrm{O}_{2}, \mathrm{OH}$ and their possible plasma species) and produce conductive Al sub-oxides, we would expect to see a negative correlation between junction quality and the partial pressures of these background impurities as recorded by our RGA. It is also possible that the competing species are some of the higher energy $N^{+}$and $N_{2}^{+}$ions, where the higher energy ions cause barrier damage. While the spectrum (580-890 nm) recorded from our Ocean Optics USB4000 spectrometer contained no unambiguous spectral peaks from $N^{+}$or $N_{2}^{+}$, a nitrogen ICP typically produces strong observable spectral signatures from $\mathrm{N}^{+}$or $\mathrm{N}_{2}^{+}$ in the UV spectrum. With this in mind, we note Ocean Optics offers commercially 
available spectrometers (similar to the one used in this study) configured to record spectra down to $165 \mathrm{~nm}$. Such a spectrometer could be directly attached to our test setup and we note the code provided in Appendix $\mathrm{V}$ can be readily modified to determine relative ratios of the intensities of any observable plasma species. Potential future work can involve investigating if and how the relative amounts of $\mathrm{N}^{+}$or $\mathrm{N}_{2}^{+}$ observed in the ICP affect AlN growth rates and junction quality.

In conclusion, we demonstrated one of our main goals in this work, the ability to realized high-quality AlN SIS mixers. However, beyond this goal, the resulting fabrication processes, mask sets, and testing procedures lay the groundwork to quickly investigate new materials and fabrication processes for potential use in higher frequency SIS based mixer circuits. We also note that the ability to in situ monitor AlN growth as determine the relative dissociation of an ICP opens the door to future studies designed to better understand the process of ICP nitridation of $\mathrm{Al}$ overlayers. These abilities, combined with the overall reduction in both fabrication and testing time, further opens the door to investigations of many new AlN growth schemes (such as those briefly investigated in Appendix II) that were previously unfeasible, in most circumstances, due to practical time constants. 


\section{Bibliography}

[1] Donald D. Arnone, Craig M. Ciesla, Alessandra Corchia, S. Egusa, Michael Pepper, J. Martyn Chamberlain, C. Bezant, Edmund H. Linfield, R. Clothier, and N. Khammo, "Applications of terahertz (THz) technology to medical imaging", pp. 209-219, September 1999.

[2] P. H. Siegel, "Terahertz technology", IEEE Transactions on microwave theory and techniques, vol. 50, no. 3, pp. 910-928, 2002.

[3] M.F. Bocko, A.M. Herr, and M.J. Feldman, "Prospects for quantum coherent computation using superconducting electronics", IEEE Transactions on Applied Superconductivity, vol. 7, no. 2, pp. 3638-3641, 1997.

[4] Gary A. Prinz, "Magnetoelectronics", Science, vol. 282, no. 5394, pp. 16601663, November 1998, PMID: 9831549.

[5] Anna Y. Herr and Quentin P. Herr, "Josephson magnetic random access memory system and method", U.S. Classification: 365/171; 365/189.15.

[6] C. Bell, G. Burnell, C. W. Leung, E. J. Tarte, D.-J. Kang, and M. G. Blamire, "Controllable josephson current through a pseudospin-valve structure", Applied Physics Letters, vol. 84, pp. 1153, February 2004.

[7] Theodore Van Duzer and Charles W. Turner, Principles of Superconductive Devices and Circuits, Prentice Hall, 2 edition, December 1998. 
[8] L. N Cooper, "Bound electron pairs in a degenerate fermi gas", Physical Review, vol. 104, no. 4, pp. 1189-1190, 1956.

[9] A. C. Rose-Innes and E. H. Rhoderick, Introduction to superconductivity, Pergamon Press, Oxford ;;New York, 2d ed. edition, 1978.

[10] J. Bardeen, L. N. Cooper, and J. R. Schrieffer, "Theory of superconductivity", Physical Review, vol. 108, no. 5, pp. 1175-1204, December 1957.

[11] B. D. Josephson, "Possible new effects in superconductive tunnelling", Physics Letters, vol. 1, no. 7, pp. 251-253, July 1962.

[12] Ghassan Yassin, Stafford Withington, Brian Ellison, and Peter Huggard, "The prospects of THz technology for ALMA 'Band 11"', in Proceedings of the 23rd International Symposium on Space Terahertz Technology, Tokyo, 2012, NRAO.

[13] John R. Tucker and Marc J. Feldman, "Quantum detection at millimeter wavelengths", Reviews of Modern Physics, vol. 57, no. 4, pp. 1055-1113, October 1985.

[14] Henry Cutler Torrey and Charles Austin Whitmer, Crystal Rectifiers, McGrawHill Book Company, 1948.

[15] P. K. Tien and J. P. Gordon, "Multiphoton process observed in the interaction of microwave fields with the tunneling between superconductor films", Physical Review, vol. 129, no. 2, pp. 647-651, January 1963.

[16] J.R. Tucker and M.F. Millea, "Photon detection in nonlinear tunneling devices", Applied Physics Letters, vol. 33, no. 7, pp. 611-613, 1978.

[17] J. R. Tucker, "Predicted conversion gain in superconductor-insulatorsuperconductor quasiparticle mixers", Applied Physics Letters, vol. 36, pp. 477-479, March 1980. 
[18] A. Kerr, S. Pan, M. Feldman, and A. Davidson, "Infinite available gain in a 115 GHz SIS mixer", Physica B+C, vol. 108, pp. 1369-1370, August 1981.

[19] W.R. McGrath, P.L. Richards, A.D. Smith, H. Van Kempen, R. A. Batchelor, D.E. Prober, and P. Santhanam, "Large gain, negative resistance, and oscillations in superconducting quasiparticle heterodyne mixers", Applied Physics Letters, vol. 39, no. 8, pp. 655-658, 1981.

[20] R. B. Bass, J. C. Schultz, A. W. Lichtenberger, R. M. Weikle, S.-K. Pan, E. Bryerton, C. K. Walker, and Jacob Kooi, "Ultra-thin silicon chips for submillimeterwave applications", 2004, p. 392.

[21] Michael Cyberey, New fabrication and characterization techniques for superconducting mixer circuits, Masters thesis, University of Virginia, Charlottesville, VA, August 2010.

[22] M. Bin, M. C. Gaidis, J. Zmuidzinas, T. G. Phillips, and H. G. LeDuc, "Low noise $1 \mathrm{THz}$ niobium superconducting tunnel junction mixer with a normal metal tuning circuit", Applied Physics Letters, vol. 68, no. 12, pp. 1714-1716, March 1996.

[23] J. W. Kooi, J. A. Stern, G. Chattopadhyay, H. G. LeDuc, B. Bumble, and J. Zmuidzinas, "Low-loss NbTiN films for THz SIS mixer tuning circuits", International journal of infrared and millimeter waves, vol. 19, no. 3, pp. 373383, 1998.

[24] T. Cecil, R. M Weikle, A. R Kerr, and A. W Lichtenberger, "Investigation of NbTiN thin films and AlN tunnel barriers with ellipsometry for superconducting device applications", IEEE Transactions on Applied Superconductivity, vol. 17, no. 2, pp. 3525-3528, June 2007. 
[25] A. R. Kerr, "Surface impedance of superconductors and normal conductors in EM simulators", NRAO Electronics Division Internal Rep, MMA Memo \#245, August 1999.

[26] W. H. Chang, "The inductance of a superconducting strip transmission line", Journal of Applied Physics, vol. 50, no. 12, pp. 8129, 1979.

[27] B. W. Maxfield and W. L. McLean, "Superconducting penetration depth of niobium", Physical Review, vol. 139, no. 5A, pp. A1515, 1965.

[28] B.W. Langley, S. M. Anlage, R. F. W. Pease, and M. R. Beasley, "Magnetic penetration depth measurements of superconducting thin films by a microstrip resonator technique", Review of Scientific Instruments, vol. 62, no. 7, pp. 1801, 1991.

[29] M. E. Cyberey, R. M. Weikle, and A. W. Lichtenberger, "On-wafer penetration depth measurements of superconducting films", in Proceedings of the 21st International Symposium on Space Terahertz Technology, University of Oxford and STFC Rutherford Appleton Laboratory, 2010.

[30] Arthur Weston Lichtenberger, Gregory S. Stronko, Jie Wang, Thomas W. Cecil, and Jian Z. Zhang, "Pentalevel resist process for the precise fabrication of small area SIS junctions", IEEE Transactions on Applied Superconductivity, vol. 19, pp. 222-225, June 2009.

[31] M. Gurvitch, M. A. Washington, and H. A. Huggins, "High quality refractory josephson tunnel junctions utilizing thin aluminum layers", Applied Physics Letters, vol. 42, no. 5, pp. 472-474, 1983.

[32] J. Kwo, G. K. Wertheim, M. Gurvitch, and D. N. E. Buchanan, "X-ray photoemission spectroscopy study of surface oxidation of $\mathrm{Nb} / \mathrm{Al}$ overlayer structures", Applied Physics Letters, vol. 40, no. 8, pp. 675-677, 1982. 
[33] A. W. Kleinsasser, W. H. Mallison, and R. E. Miller, "Nb/AlN/Nb josephson junctions with high critical current density", Applied Superconductivity, IEEE Transactions on, vol. 5, no. 2, pp. 2318-2321, 1995.

[34] Z. Wang, Y. Uzawa, and A. Kawakami, "High current density NbN/AlN/NbN tunnel junctions for submillimeter wave SIS mixers", Applied Superconductivity, IEEE Transactions on, vol. 7, no. 2, pp. 2797-2800, 1997.

[35] T. Shiota, T. Imamura, and S. Hasuo, "Nb josephson junction with an AlNx barrier made by plasma nitridation", Applied physics letters, vol. 61, no. 10, pp. 1228-1230, 1992.

[36] A. B Kaul, A. W Kleinsasser, B. Bumble, H. G LeDuc, and K. A Lee, "Aluminum nitride tunnel barrier formation with low-energy nitrogen ion beams", Journal of materials research, vol. 20, no. 11, pp. 3047-3053, 2005.

[37] D. Van Vechten and J. F Liebman, "Considerations on the selection of artificial tunneling barriers", Journal of Vacuum Science 83 Technology A: Vacuum, Surfaces, and Films, vol. 3, no. 4, pp. 1881-1883, 1985.

[38] T. W Cecil, M. E Cyberey, R. E Matthews, J. Z Zhang, and A. W Lichtenberger, "Development of Nb/Al-AlN/NbTiN SIS junctions with ICP nitridation", IEEE transactions on applied superconductivity, vol. 19, no. 3, pp. 409, 2009.

[39] Thomas William Cecil, New fabrication techniques for AIN-based superconductor tunnel junctions, Ph.D. dissertation, University of Virginia, Charlottesville, VA, May 2008.

[40] CCR Technology, "COPRA GS/GSE plamsa beam source user manual", September 2003. 
[41] T. Zijlstra, C. F. J. Lodewijk, N. Vercruyssen, F. D. Tichelaar, D. N. Loudkov, and T. M. Klapwijk, "Epitaxial aluminum nitride tunnel barriers grown by nitridation with a plasma source", Applied Physics Letters, vol. 91, no. 23, pp. 233102-233102-3, December 2007.

[42] Th. Muhge, K. Westerholt, H. Zabel, N. N. Garif'yanov, Yu. V. Goryunov, I. A. Garifullin, and G. G. Khaliullin, "Magnetism and superconductivity of Fe/Nb/Fe trilayers", Physical Review B, vol. 55, no. 14, pp. 8945-8954, April 1997.

[43] Wenlei Shan, Shinichiro Asayama, Mamoru Kamikura, Takashi Noguchi, Shengcai Shi, and Yutaro Sekimoto, "Development of a 385-500GHz SIS mixer for ALMA band 8", May 2005, pp. 175-180.

[44] R.B. Bass, J.Z. Zhang, and A.W. Lichtenberger, "Machine-aligned fabrication of submicron SIS tunnel junctions using a focused ion beam", IEEE Transactions on Applied Superconductivity, vol. 9, no. 2, pp. 3240-3243, June 1999.

[45] H. G. LeDuc, A. Judas, S. R. Cypher, B. Bumble, B. D. Hunt, and J. A. Stern, "Submicron area NbN/MgO/NbN tunnel junctions for SIS mixer applications", Magnetics, IEEE Transactions on, vol. 27, no. 2, pp. 3192-3195, 1991.

[46] A.W. Lichtenberger, D.M. Lea, C. Li, F.L. Lloyd, M.J. Feldman, R.J. Mattauch, S.-K. Pan, and A.R. Kerr, "Fabrication of micron size Nb/Al-Al2O3/Nb junctions with a trilevel resist liftoff process", IEEE Transactions on Magnetics, vol. 27, no. 2, pp. 3168-3171, March 1991.

[47] A. W. Lichtenberger, D. M. Lea, and F. L. Lloyd, "Investigation of etching techniques for superconductive $\mathrm{Nb} / \mathrm{Al}-\mathrm{Al} 2 \mathrm{O} 3 / \mathrm{Nb}$ fabrication processes", IEEE Transactions on Applied Superconductivity, vol. 3, pp. 2191-2196, March 1993. 
[48] William W. Clark, Jian Z. Zhang, and Arthur W. Lichtenberger, "Ti quadlevel resist process for the fabrication of nb SIS junctions", Applied Superconductivity, IEEE Transactions on, vol. 13, no. 2, pp. 115-118, 2003.

[49] Oxford Instruments, "Plasmalab system 100".

[50] Roy Matthews, Improving Insulation Methods in New and Existing Fabrication Processes, Masters thesis, University of Virginia, Charlottesville, VA, 2012.

[51] K.R. Williams, K. Gupta, and M. Wasilik, "Etch rates for micromachining processing-part II", Journal of Microelectromechanical Systems, vol. 12, no. 6, pp. $761-778$, December 2003.

[52] Brian N Chapman, Glow discharge processes: sputtering and plasma etching, Wiley, New York, 1980.

[53] "ACT 935 UP datasheet", August 2013.

[54] Dallas Millard Lea, Integrated Test Structures for Characterization of Thin Films for Superconductor-Insulator-Superconductor Devices and Circuits, Ph. d., University of Virginia, 1996, Description: viii, 154 leaves : ill. ; $29 \mathrm{~cm}$ Other format: Also available online through Digital Dissertations Local note: Dissertation Local note: Department of] Electrical Engineering.

[55] E. J. Cukauskas, M. Nisenoff, D. W. Jillie, H. Kroger, and L. N. Smith, "High quality niobium nitride-niobium josephson tunnel junctions", Magnetics, IEEE Transactions on, vol. 19, no. 3, pp. 831-834, 1983.

[56] Hiroyuki Mori, Mikio Hirano, Yuji Hatano, Ushio Kawabe, Hirojo Yamada, and Yoshinobu Tarutani, "Fabrication of high-quality Nb/AlOx/Nb josephson junctions", Electronics and Communications in Japan (Part II: Electronics), vol. 73 , no. 4, pp. 83-96, 1990. 
[57] John A. Thornton, "The microstructure of sputter-deposited coatings", Journal of Vacuum Science \&3 Technology, vol. 4, no. 6, pp. 3059-3065, November 1986.

[58] David M.Pozar, Microwave Engineering, 3Rd Ed, Wiley India Pvt. Limited, September 2009.

[59] Stephen A. Campbell, The Science and Engineering of Microelectronic Fabrication, Oxford University Press, 2001.

[60] P. Drude, "Ueber oberflachenschichten. i. theil", Annalen der Physik, vol. 272, no. 2, pp. 532-560, 1889 .

[61] Frank L. McCrackin, Elio Passaglia, Robert R. Stromberg, and Harold L. Steinberg, "Measurement of the thickness and refractive index of very thin films and the optical properties of surfaces by ellipsometry", Journal of research of the national bureau of standards-A. physics and chemistry A, vol. 67, 1963.

[62] Alexandre Rothen, "The ellipsometer, an apparatus to measure thicknesses of thin surface films", Review of Scientific Instruments, vol. 16, no. 2, pp. 26-30, February 1945.

[63] Milton Ohring, Materials Science of Thin Films, Academic Press, October 2001.

[64] Inc. J.A. Woollam Co., "CompleteEASE data analysis manual", 2009.

[65] Harland G. Tompkins and William A. McGahan, Spectroscopic Ellipsometry and Reflectometry: A User's Guide, Wiley, March 1999.

[66] Neil W. Ashcroft and N. David Mermin, Solid State Physics, Cengage Learning India Private Limited, 2011.

[67] Georg Haas, Physics of thin films: advances in research and development, Academic Press, 1963. 
[68] Harland G. Tompkins, A user's guide to ellipsometry, Academic Press, 1993.

[69] Maria Losurdo and Kurt Hingerl, Ellipsometry at the nanoscale, Springer, Berlin; New York, 2013.

[70] American Institute of Physics, American Institute of Physics handbook: Section editors: Bruce H. Billings [and others] Coordinating editor: Dwight E. Gray, McGraw-Hill, 1972.

[71] S. Rudra, T. WÃ€chtler, M. Friedrich, S. J. Louis, C. Himcinschi, S. Zimmermann, S. E. Schulz, S. Silaghi, C. Cobet, N. Esser, T. Gessner, and D. R. T. Zahn, "Spectroscopic ellipsometry study of thin diffusion barriers of TaN and ta for cu interconnects in integrated circuits", physica status solidi (a), vol. 205, no. 4, pp. 922-926, 2008.

[72] L. Roskovcova and J. Pastrmak, "The urbach absorption edge in ALN", Czechoslovak Journal of Physics B, vol. 30, no. 5, pp. 586-591, May 1980.

[73] H. Kohlstedt, F. Konig, P. Henne, N. Thyssen, and P. Caputo, "The role of surface roughness in the fabrication of stacked $\mathrm{Nb} / \mathrm{Al}-\mathrm{AlOx} / \mathrm{Nb}$ tunnel junctions", Journal of Applied Physics, vol. 80, no. 9, pp. 5512, 1996.

[74] L. G. SCHULZ and F. R. TANGHERLINI, "Optical constants of silver, gold, copper, and aluminum. II. the index of refraction n", Journal of the Optical Society of America, vol. 44, no. 5, pp. 362-367, May 1954.

[75] M. A. Ordal, Robert J. Bell, R. W. Alexander, L. L. Long, and M. R. Querry, "Optical properties of fourteen metals in the infrared and far infrared: $\mathrm{Al}, \mathrm{co}$, cu, au, fe, pb, mo, ni, pd, pt, ag, ti, v, and w.", Applied Optics, vol. 24, no. 24, pp. 4493-4499, December 1985. 
[76] C. M. Herzinger, B. Johs, W. A. McGahan, J. A. Woollam, and W. Paulson, "Ellipsometric determination of optical constants for silicon and thermally grown silicon dioxide via a multi-sample, multi-wavelength, multi-angle investigation", Journal of Applied Physics, vol. 83, no. 6, pp. 3323-3336, March 1998.

[77] Jebreel M. Khoshman and Martin E. Kordesch, "Optical characterization of sputtered amorphous aluminum nitride thin films by spectroscopic ellipsometry", Journal of Non-Crystalline Solids, vol. 351, no. 40-42, pp. 3334-3340, October 2005.

[78] R.B. Bass, L.T. Lichtenberger, and A.W. Lichtenberger, "Effects of substrate preparation on the stress of nb thin films", IEEE Transactions on Appiled Superconductivity, vol. 13, no. 2, pp. 3298-3300, June 2003.

[79] A. Endo, T. Noguchi, M. Kroug, T. Tamura, and H. Inoue, "Atomic nitrogen source for the formation of aluminum nitride tunnel barrier SIS junctions", Physica C: Superconductivity, vol. 469, no. 15-20, pp. 1589-1592, October 2009.

[80] Robert T DeHoff, Thermodynamics in materials science, CRC/Taylor \& Francis, Boca Raton, 2006.

[81] John Emsley, The elements, Clarendon Press ; Oxford University Press, Oxford; New York, 1998.

[82] B. deB Darwent, United States , National Bureau of Standards, United States, and Department of Commerce, Bond dissociation energies in simple molecules, U.S. National Bureau of Standards; for sale by the Supt. of Docs., U.S. Govt. Print. Off., [Washington, 1970.

[83] J. D. Cox, Donald D. Wagman, and Vadim Andreevich Medvedev, CODATA key values for thermodynamics, Chem/Mats-Sci/E, 1989. 
[84] H. Carrere, A. Arnoult, E. Bedel-Pereira, and A. Ricard, "Influence of radio frequency plasma cell conditions on the incorporation of nitrogen into GaAsN and GaInAsN", Journal of Vacuum Science 83 Technology B, vol. 22, no. 5, pp. 2448-2453, 2004.

[85] K. Sasaki, H. Kokubu, D. Hayashi, and K. Kadota, "Development of a compact nitrogen radical source by helicon-wave discharge employing a permanent magnet", Thin Solid Films, vol. 386, no. 2, pp. 243-247, 2001.

[86] Costel Biloiu, Earl E. Scime, Ioana A. Biloiu, and Xuan Sun, "Nitrogen dissociation degree in the diffusion region of a helicon plasma source obtained by atomic lines to molecular band intensities ratio", Journal of Applied Physics, vol. 102, no. 5, pp. 053303-053303-11, September 2007.

[87] Alf Lofthus and Paul H. Krupenie, "The spectrum of molecular nitrogen", Journal of physical and chemical reference Data, vol. 6, no. 1, pp. 113-307, 1977.

[88] M Razouki and Manfred Weiler, "COPRA plasma source characteristics - internal document", December 2012.

[89] J. Hopwood, "Review of inductively coupled plasmas for plasma processing", Plasma Sources Science and Technology, vol. 1, no. 2, pp. 109, 1992.

[90] C.E. Moore, "Selected tables of atomic spectra, atomic energy levels and multiplet tables - n i, n II, n III", Tech. Rep., Nat. Bur. Stand, U.S., 1975.

[91] "Maya2000 pro deep UV spectrometer product sheet", 2012.

[92] Martin Sternitzke, "Growth of oxide layers on thin aluminum nitride samples measured by electron energy-loss spectroscopy", Journal of the American Ceramic Society, vol. 76, no. 9, pp. 2289-2294, 1993. 
[93] ALMA Press Release, "ALMA inauguration heralds new era of discovery", March 2013.

[94] Christopher Groppi, Christopher Walker, Craig Kulesa, Dathon Golish, Jenna Kloosterman, Sander Weinreb, Glenn Jones, Joseph Barden, Hamdi Mani, and Tom Kuiper, "SuperCam: a 64 pixel heterodyne array receiver for the $350 \mathrm{GHz}$ atmospheric window", in 20th Int. Space Terahertz Technol. Symp, 2009, pp. 90-96.

[95] Alistair Christopher Rose-Innes, Low temperature laboratory techniques: the use of liquid helium in the laboratory, English Universities Press, [London], 1973.

[96] Ivar Giaever, "Study of superconductors by electron tunneling", Physical Review, vol. 122, no. 4, pp. 1101-1111, 1961.

[97] A. R. Kerr and R. Groves, "Measurements of copper heat straps near $4 \mathrm{k}$ with and without apiezon-n grease", October 2006.

[98] A. Darlinski and J. Halbritter, "Angle-resolved XPS studies of oxides at NbN, NbC, and nb surfaces", Surface and Interface Analysis, vol. 10, no. 5, pp. 223-237, June 1987.

[99] N.N. Iosad, T.M. Klapwijk, S.N. Polyakov, V.V. Roddatis, E.K. Kov'ev, and P.N. Dmitriev, "Properties of DC magnetron sputtered nb and NbN films for different source conditions", IEEE Transactions on Applied Superconductivity, vol. 9, no. 2, pp. 1720 -1723, June 1999.

[100] H. Myoren, T. Shimizu, T. Iizuka, and S. Takada, "Properties of NbTiN thin films prepared by reactive DC magnetron sputtering", Applied Superconductivity, IEEE Transactions on, vol. 11, no. 1, pp. 3828-3831, 2001. 


\section{Appendix I}

\section{On-wafer Cryogenic Screening}

\section{Cryogenic DC Probing of SIS Mixers}

Chapter 2 introduced previous work where our research group successfully demonstrated the use of our modified Lakeshore CPX 1.5K Probe Station for cryogenic microwave testing (up to $50 \mathrm{GHz}$ ). The penetration depth of our $\mathrm{Nb}$ and NbTiN films was determined using RF test structures fabricated on $300 \mu m$ thick Si substrates utilizing a pair of Picoprobe 50A-GSG-150 RF probes. The obtained results matched well with both BCS theory and with results published in the literature for similar films cooled to near $4.2 \mathrm{~K}$ [29].

It would be very advantageous to be able to extend the capabilities of the probe system to measure the DC IV characteristics of individual mixers on-wafer. This would will allow the screening of individual mixers before the lengthy backside process, as well as monitoring of possible variations in $R_{N} A$ or $R_{S G} / R_{N}$ across the wafer. This would also provide a notable advantage for projects that require a large number of high-quality well-matched junctions, including multi-pixel receivers, multi-telescope arrays, and balanced heterodyne receiver schemes. A limiting factor of on-wafer IV measurements that may increase device temperature, is that they 
must be performed at cryogenic temperatures using a four point measurement, providing additional thermal sources when compared to a two port RF measurement.

Additional considerations also need to be made with respect to electrical charges when testing small area Nb based SIS junctions, which are nominally biased to 10 $\mathrm{mV}$ and $<1 \mathrm{~mA}$. As we have shown in Chapter 2, SIS junctions are easily damaged by static discharge during testing. For these reasons, a switch box providing make-before-break that grounds all four probes before connecting to the bias box is designed, similar to that used on our current POGO pin dip stick setup.

\section{Experimental Setup}

Testing was performed using a Lakeshore CPX 1.5K Probe Station, with temperature measured through a LakeShore Model 332 temperature controller and Model DT670-SD-1.4H calibrated silicon diode mounted in the sample stage. The sample stage as well as the surrounding radiation shield are held under high vacuum and cooled through an open cycle flow of liquid helium from a pressurized dewar. A set of four Lakeshore ZN50R-CVT DC probes with CuBe tips were used to make DC connections with the test devices. The Band-6 mixer chips described in Chapter 3 were mounted to a $300 \mu m$ thick Si carrier via a small amount of Apiezon N Grease dissolved in trichloroethylene (TCE). Additionally, the POGO chips described in Chapter 4 were also tested. Both the Si carrier containing Band-8 mixer chips and individual POGO chips were mounted to the Au plated 4.2 cold stage using a thin layer of Apiezon L Grease. The Au beamleads were pressed into the carrier during testing to provide a thermal conduction path to cool the chips. During initial testing, the devices failed to exhibit signs of superconductivity. After contacting Lakeshore, the following modifications were implemented to enhance wafer cooling. 


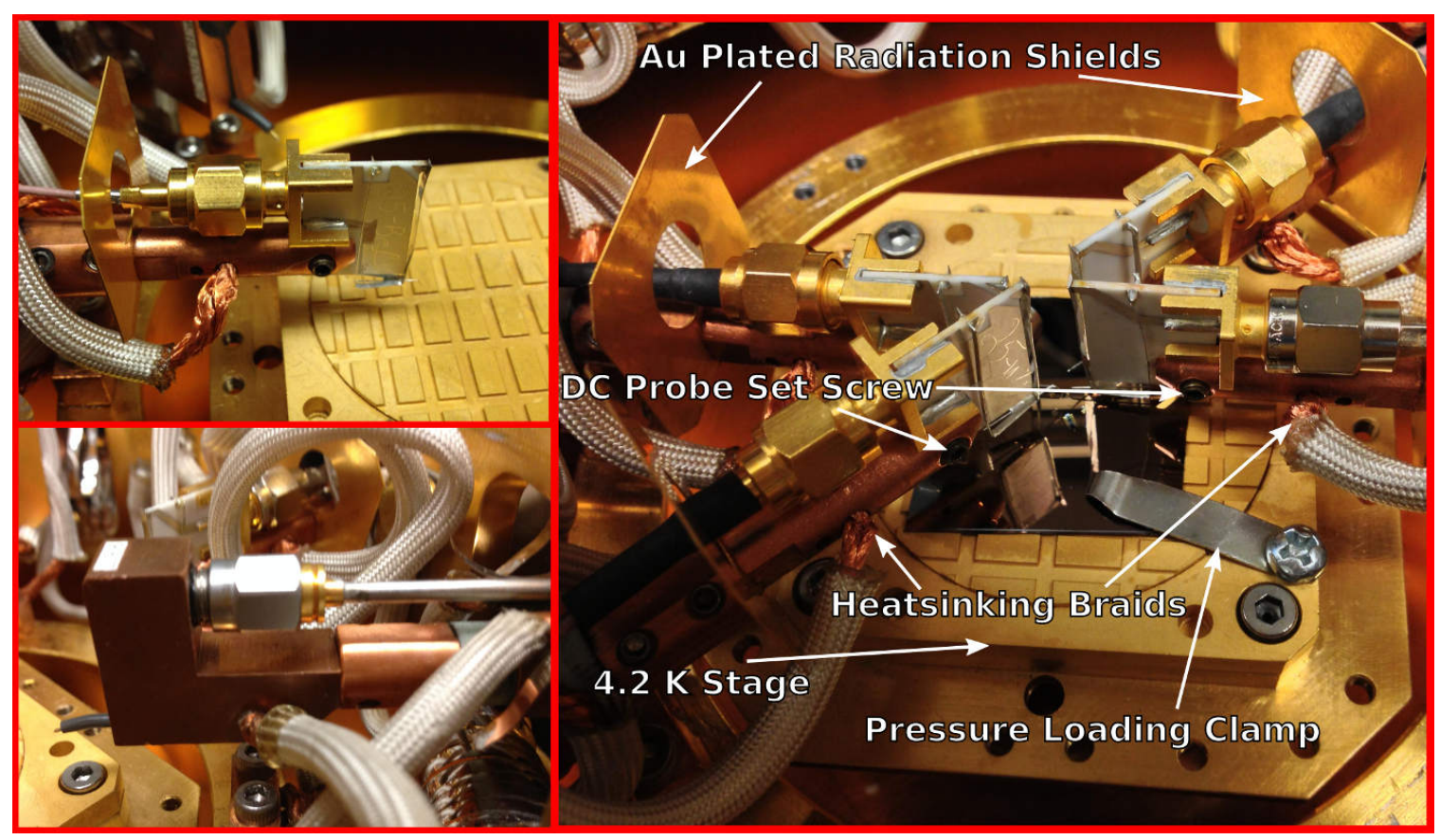

Figure I.1: Top Left: Lakeshore ZN50R-CVT DC probe mounted to probe holder. Bottom Left: Picoprobe 50A-GSG-150 RF probe. Right: Band-8 mixers mounted to $\mathrm{Si} / \mathrm{SiO}_{2}$ carrier, carrier heatsunk to $4.2 \mathrm{~K}$ cold stage via pressure loaded Apiezon $\mathrm{N}$ Grease.

We note that for initial testing, no clamping force was used between the sample and stage. Under vacuum, thermal conductivity between the sample and stage is made through small contact points and is generally assumed proportional to contact force [95]. With this assumption in mind, pressure loading clamps were added to the stage to provide an increased contact force between the sample and $4.2 \mathrm{~K}$ cold stage, as shown in Figure I.1. Additionally, we note that the electrical connections on the rear of the probes are made through vacuum feed throughs, which are at ambient temperature. Behind the probes, Au plated placards were installed, which are cooled through metal to metal contact with the probe arm and serve to block radiation emitted from the higher temperature cabling, shown in Figure I.1.

For DC biasing of the probes, a simple bias box was designed, the schematic of which is shown in Figure I.2. Keeping in mind how static charges may damage SIS devices, a four pole make-before-break double throw switch is used to simultaneously 
ground all four probes while making electrical contact to the device. After contact is made, the switch connects the probes to both the DC bias box and data logger. DC biasing is achieved using a battery powered voltage limited DC current supply with a max available current of $\pm 2 \mathrm{~mA}$. The junction voltage is recorded directly from two of the probes, the bias current is determined from the voltage across a $50 \Omega$ resistor in series with the current supply, and the data is recorded using a battery powered Graphtec GL900 data logger through a pair of shielded BNC connections. The circuit was fabricated inside an $\mathrm{Al}$ project box, using isolated ground $\mathrm{BNC}$ connections to the outside.

\section{Results}

Initial DC testing of Band-8 mixer chips mounted via Apiezon N-grease and pressure loading via stainless steel clamps are shown on the left of Figure I.3. The temperature was measured using the aforementioned temperature sensor mounted inside the sample stage, and was varied using resistive heating of the stage controlled by the temperature controller. The resulting IV characteristics are a classic example of an under-cooled SIS junction. A superconductor's energy gap varies as a function of temperature, as shown in Figure I.4, and for temperatures near $\mathrm{T}_{c}$, the relationship follows:

$$
\frac{\Delta(T)}{\Delta_{0}} \approx\left(1-\frac{T}{T_{c}}\right)^{(1 / 2)},
$$

where $\Delta_{0}$ is the energy gap at $0 \mathrm{~K}[10,96]$. Noting this, the measured energy gap of our devices gives a means of inferring the actual device temperature, which may vary from the bulk stage temperature. We calculated a 2-3 K temperature differential 


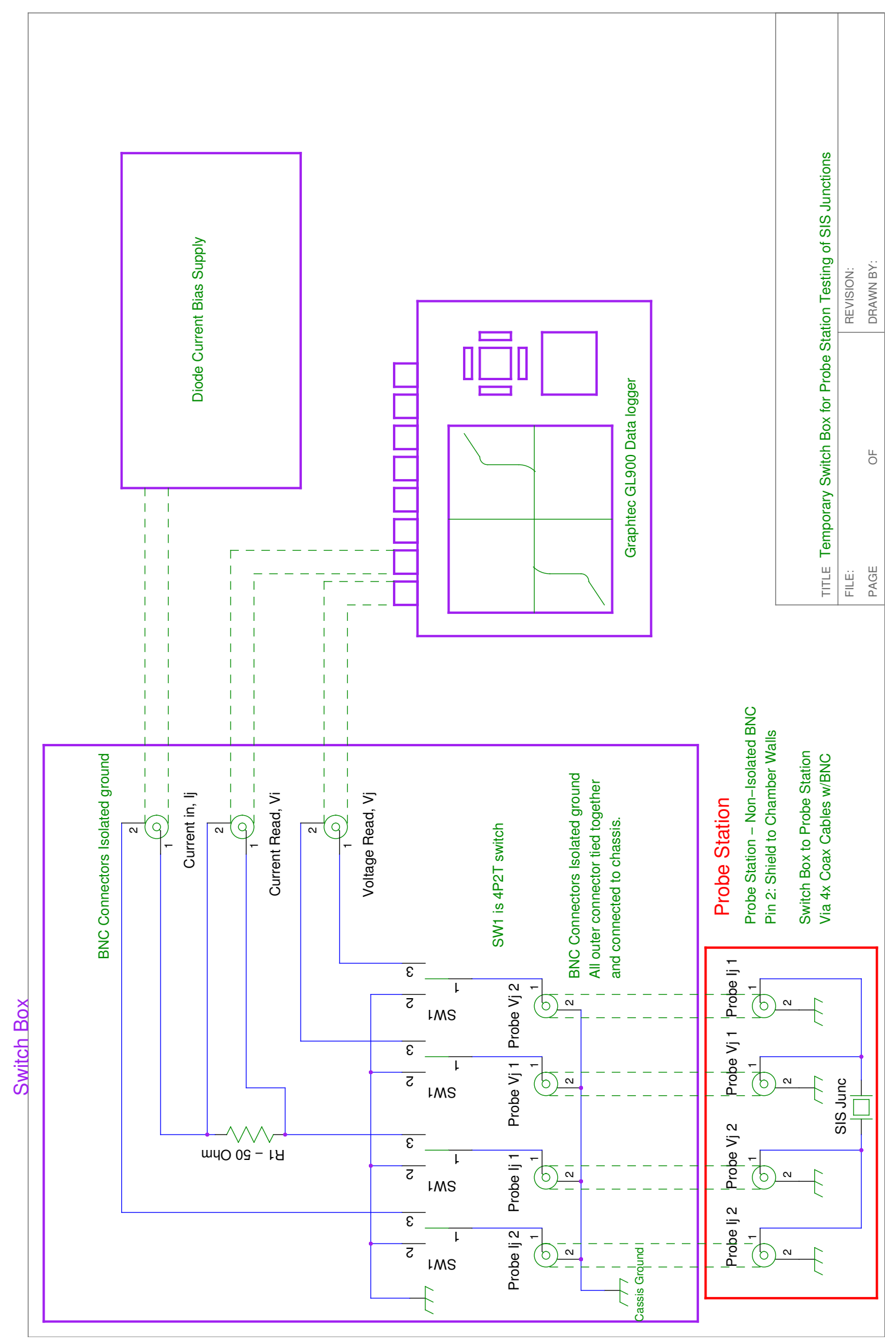

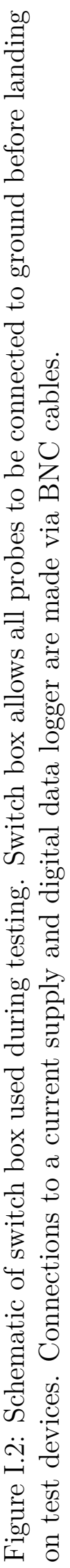


T3-613 - CryoStation

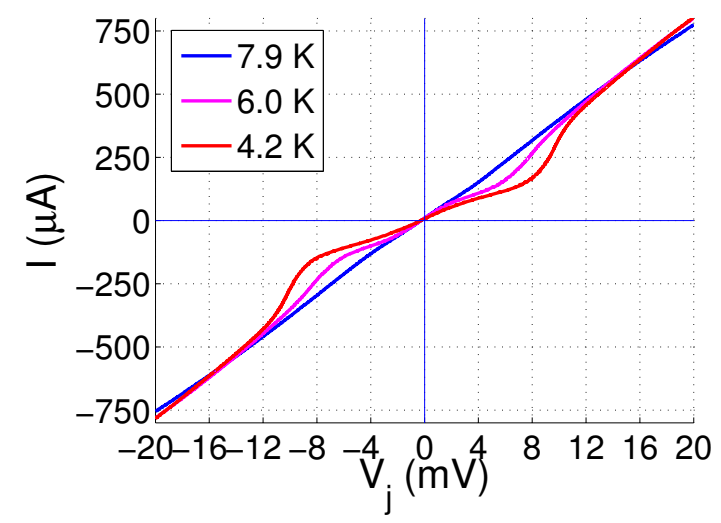

Compare TIM at $2.7 \mathrm{~K}$

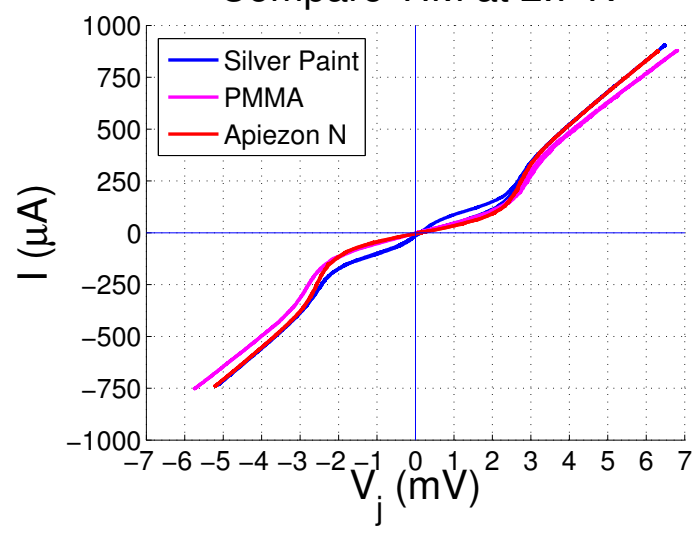

Figure I.3: Left: IV measurements of Band-8 mixer chips mounted to Si carrier across three cold stage temperature. Right: IV measurements of previous tested POGO chips mounted with three different TIMs. Cold stage temperature was reduced below $4.2 \mathrm{~K}$ by pulling a vacuum on the exhaust of the cryostation.

between recorded stage temperature and actual device temperature during the four point measurements.

With these results in mind, we were unsure if lack of conduction through the pressed beam-leads was to blame for the increased temperature, compounded through the use of Apiezon N grease as a TIM. The total thermal resistance would be that of the substrate cold stage interface, as well as the beamlead substrate interface. While $\mathrm{N}$ grease can help thermal conduction through filling in voids where no conduction can take place, it has been shown to increase thermal resistance through blocking the point contacts which may occur for small overlaps [97]. Noting this, we explored the use of alternative TIM materials. Three POGO chips, with known good quality SIS IV characteristics from dip testing in liquid helium were mounted to the cold stage using colloid silver paint, PMMA, and Apiezon N grease.

Noting the probe station is open cycle, cooling is provided through liquid helium supplied from a dewar with an exhaust open to ambient, for some experiments we reduced the stage temperature past $4.2 \mathrm{~K}$ by attaching a rough pump to the exhaust to reduce pressure. Through use of pumping, a minimum stage pressure of $2.7 \mathrm{~K}$ was achieved during this TIM testing. The results of all three TIMs at $2.7 \mathrm{~K}$ are shown 


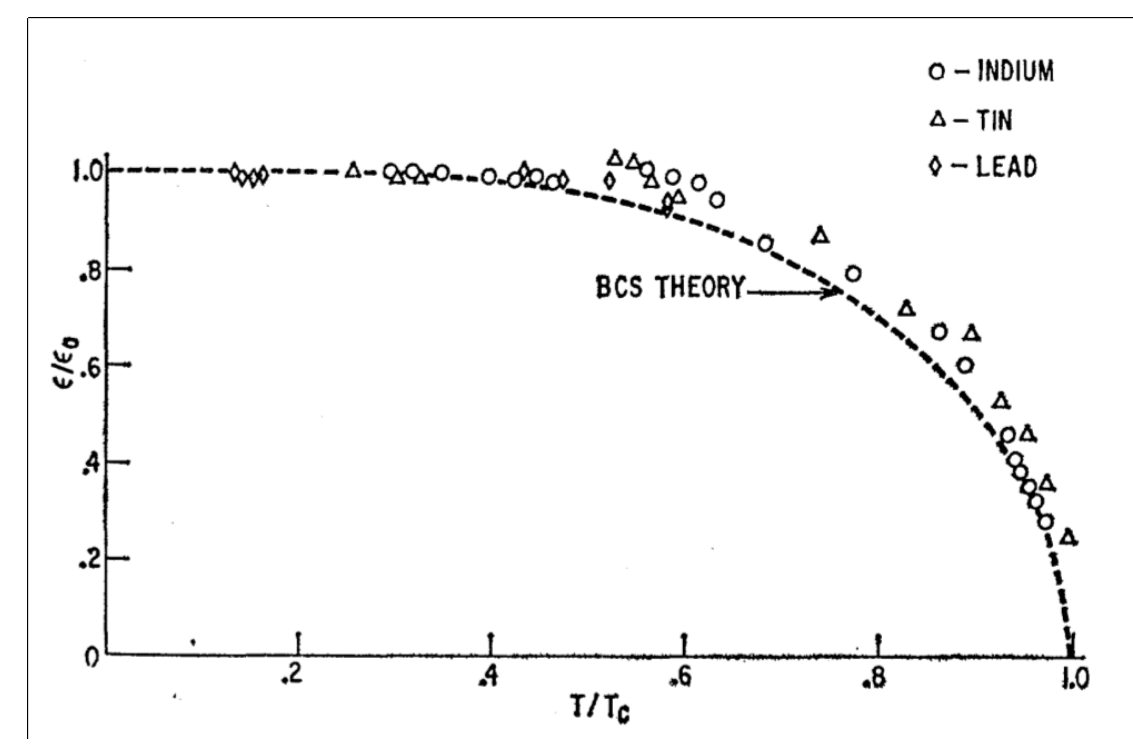

Figure I.4: Theoretical and experimentally observed dependence on energy gap, $\epsilon$, compared to its value at $0 \mathrm{~K}, \epsilon_{0}$, as a function of reduced temperature, $T / T_{c}$, for In, $\mathrm{Pb}$, and Sn. Graph taken from [96].

on the right of Figure I.3. Compared to the previous test, a slightly increased gap voltage is observed, but despite cooling the stage to $2.7 \mathrm{~K}$, the reduced gap indicates a device temperature of $\sim 6 \mathrm{~K}$, or a $\sim 3 \mathrm{~K}$ temperature gradient. Interestingly, the difference in gap between the three TIMs is negligible. Possible explanations to the temperature gradient observed while DC testing are discussed in the next section.

\section{Conclusions and Future Work}

We have successfully performed RF measurements at temperatures close to $4.2 \mathrm{~K}$; however, the current state of DC testing of SIS test devices using our CPX 1.5K probe station and 4 DC probes, despite all modification suggested by Lakeshore, still produces a temperature gradient of $\sim 3 \mathrm{~K}$ between the cold stage and device temperature. We believe a major difference between our RF and DC measurements is thermal loading of our devices through the hot DC probes. In terms of thermal loading, a difference between the RF and DC probes provided by Lakeshore, in our 


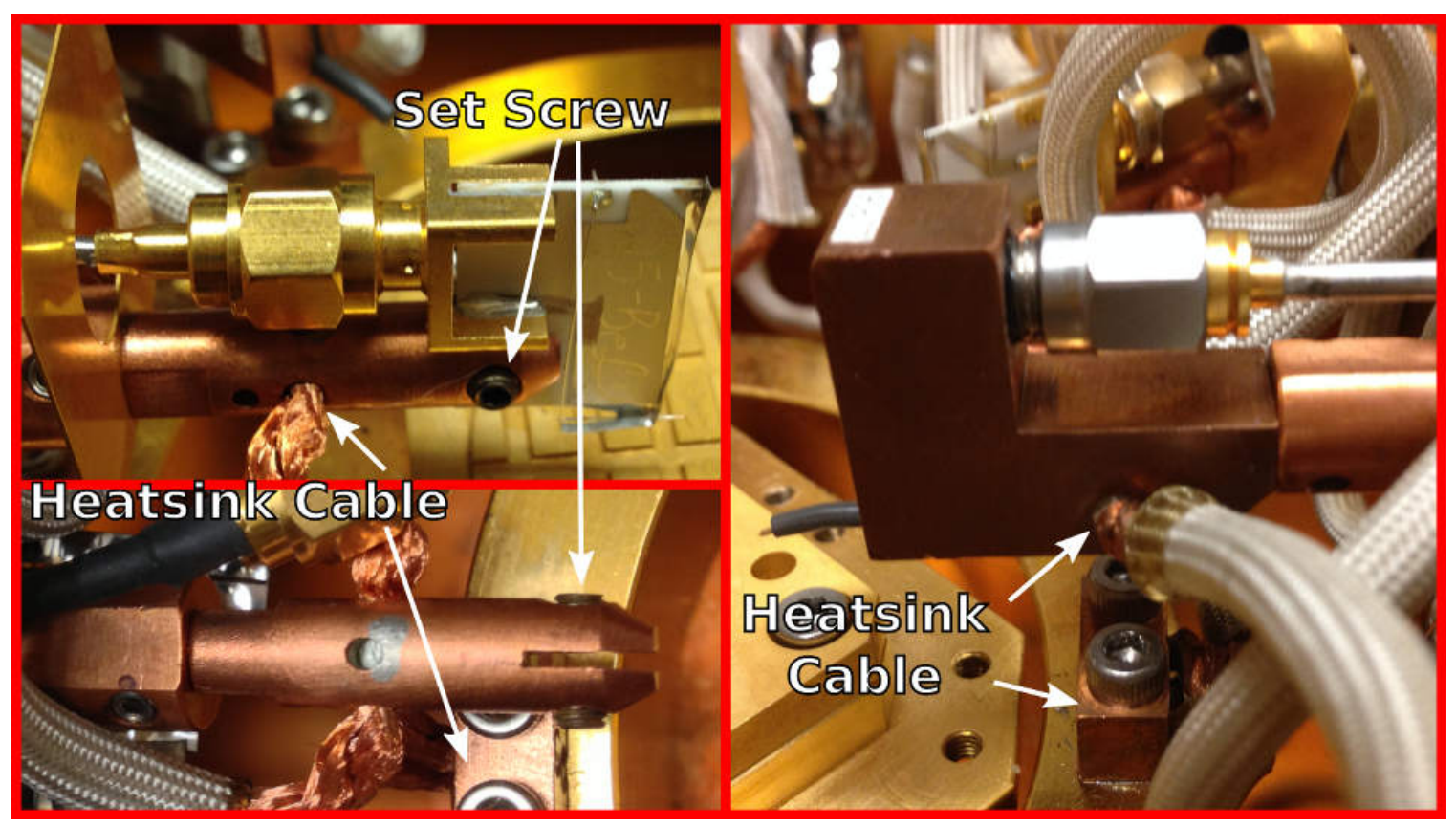

Figure I.5: Left: Lakeshore ZN50R probe showing heatsinking of probe arm to $4.2 \mathrm{~K}$ stage via copper cables, however, the actual DC card is only physically attached to the probe arm via two set screws. Right: GGB RF probe showing heatsinking to 4.2 K stage via copper cables. Probe tip is physically attached to large copper body.

particular configuration, is that the DC probes are fabricated on a ceramic substrate, and physically heatsunk to the sample holder only through the small point contact of a set screw. The voids between the ceramic card and copper sample holder may be filled with Apiezon N-grease, but N-grease undergoes a glass transition at $200 \mathrm{~K}$ when not pressure loaded, and becomes a terrible thermal conductor at low temperatures. However, the RF probes are fabricated inside a copper body, bypassing the use of a probe holder, and are physically heatsunk by a soldered copper cable attached to the 4.2 K stage. Pictures of the two probes are shown in Figure I.5, demonstrating the differences.

Our suggested solution is to use similar RF probes for 4 point DC measurements. With two isolated pins per probe, only two probes are required for a 4 point measurement. The problem with the currently available RF probes is that they are in a ground-signal-ground (GSG) coplanar waveguide (CPW) configuration. The ground 
pins are electrically connected to the probe body, which is electrically connected to the grounded probe station chassis. As such, landing the probes on a single DC electrode will electrically ground the electrode, making DC measurements impossible. The Lakeshore probes are manufactured by GGB Industries who offer many configurations, including ground-signal-signal-ground (GSSG) probes, which provide two independent signal contact points on a single probe. If we can obtain an RF probe modified in a similar way with copper braids to our current Picoprobe 50A-GSG-150 RF probes, but using a GSSG configuration, the two ground pins can be removed (or simply bent up with tweezers), leaving two signal pins for a four point measurement.

The use of such a modified RF probe offers significant advantages, firstly the probes will be much better thermally heatsunk than the ZN50R DC probes. Secondly, when 4 separate DC probes and pressure loading of the sample via clips is used, there are often areas on the sample where all four probes cannot land due to physical interference with the clips. With two laterally aligned probes, instead of four probes at 90 degree angles, the clips can more easily be positioned out of the way. Lastly, the use of two probes rather than four expedites probing, which will prove itself useful if many devices are to be rapidly tested.

We also note the circuit shown in Figure I.2 was designed as a temporary implementation in order to perform these tests without damaging our SIS junctions. A new switch box was designed, which can directly interface with the low-noise SIS bias boxes used by NRAO. The schematics of this box are shown in Figure I.6. 


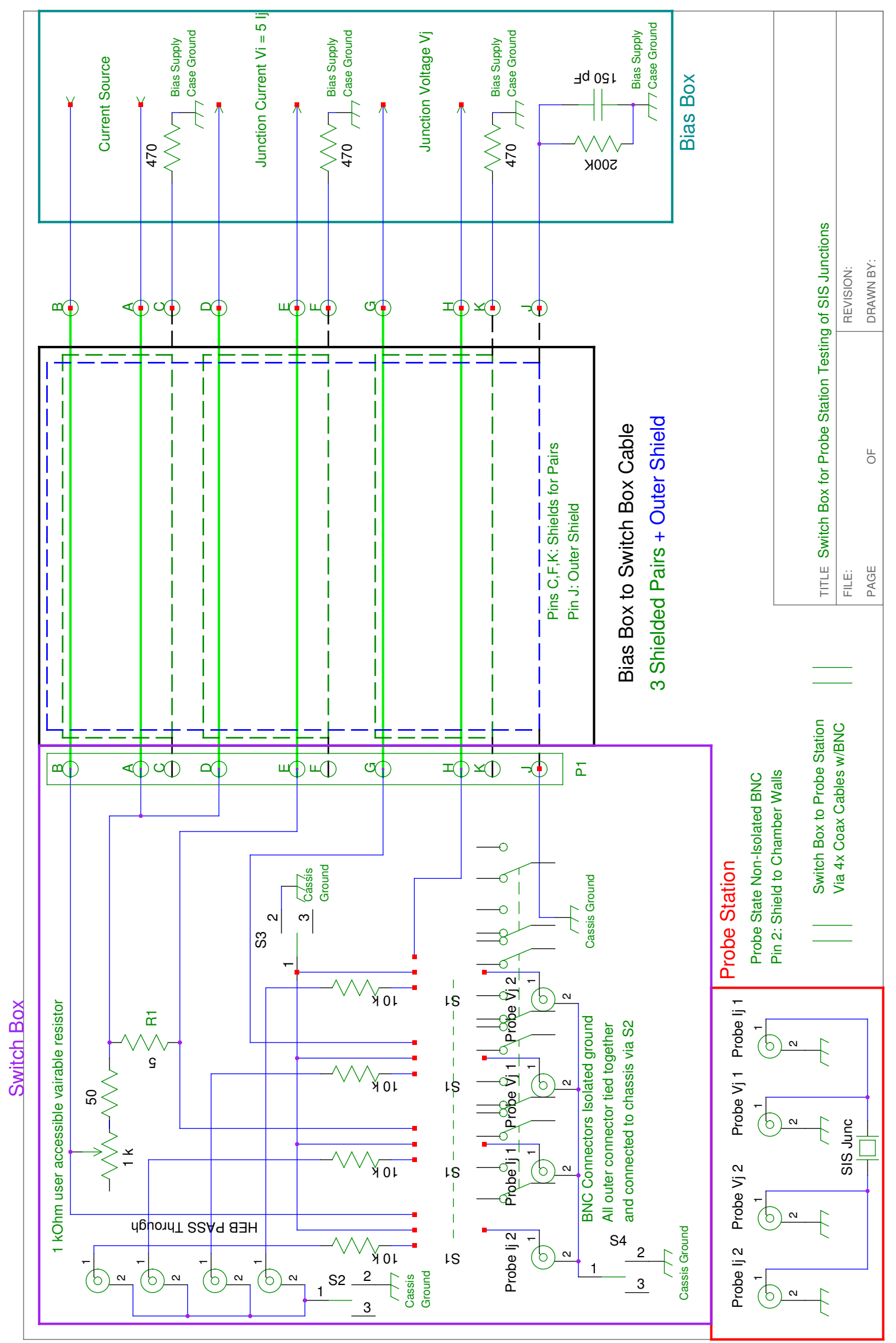

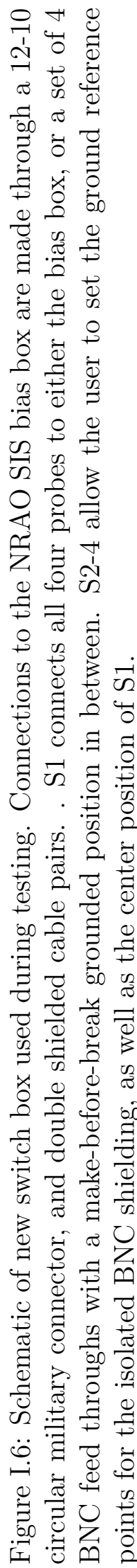




\section{Appendix II}

\section{Investigation of Directly Deposited AlN Barriers}

\section{Direct deposition of AlN barriers}

The work in this dissertation primary focused on Nb/Al-AlN/Nb SIS tunnel junctions with AlN barriers formed by means of ICP nitridiation of an Al overlayer. While ICP nitridation of $\mathrm{Al}$ overlayers produced good quality junctions with reasonable control over $R_{n} A$ (allowing our research group to realize low-noise 385-500 GHz ALMA Band8 AlN-based mixers fabricated on $3 \mu m$ Si substrates), we note the use of Al overlayers is not compatible with higher bandgap NbTiN base electrodes. An alternate method of AlN barrier formation is through the direct deposition of AlN, through reactive sputtering of an $\mathrm{Al}$ target. This appendix presents our research group's initial study on the potential use of directly sputtered AlN barriers on both Nb and NbTiN base electrodes. We note a driving force for future work beyond this initial study is that an all NbTiN-AlN based mixer would be beneficial SIS mixer design, as it would allow theoretical operation up to $2.4 \mathrm{THz}$.

In this work, SIS tunnel junctions were fabricated and DC tested at cryogenic 
temperatures using a similar process described in Chapter 4. All trilayer for DC test samples was deposited on $330 \mu \mathrm{m}$ thick, $50 \mathrm{~mm}$ diameter Si wafers with 1500-2500 $\AA$ of thermally grown $\mathrm{SiO}_{2}$. All wafers were cleaned in situ prior to deposition via Ar ion milling and both chambers were allowed to reach a base pressure in the $10^{-8}$ Torr range with a partial pressure of water in the $10^{-9}$ Torr range. Nb films were deposited by DC magnetron sputtering in Ar with an applied power of $180 \mathrm{~W}$ and at a pressure of 5.0-6.5 mTorr. The direct deposition of AlN barriers was achieved through DC magnetron sputtering of an Al target in 2.0 mTorr of $99.9999 \%$ purity $\mathrm{N}_{2}$ with an applied power of $50 \mathrm{~W}$. The barrier thickness was controlled utilizing a water-cooled rotating sample holder to sweep the wafer at a constant speed through the AlN flux produced by the sputtering gun. We note, the wafer holder is presented behind pie shaped cutouts in a deposition shield to define a fixed deposition geometry field of view to the gun.

We first needed to determine if a directly sputtered AlN barrier fully covered our base electrode $\mathrm{Nb}$ layer, as pinholes would allow leakage current to flow through the barrier. With this in mind, chemical analysis was performed using utilizing X-ray Photoelectron Spectroscopy (XPS). XPS measures the core level electron binding energies, revealing the chemical nature of the first $\sim 10 \mathrm{~nm}$ of a sample. It can be used to detect the formation of Nb-oxides and hence determine whether a barrier fully covers a surface, preventing oxidation of underlying films. Two sample were produced, the first consisting of a $2000 \AA$ thick Nb base layer capped with a $\sim 12 \AA$ thick $\mathrm{Al}$ overlayer and the second with $\sim 15 \AA$ thick reactively sputtered AlN overlayer. The samples were next exposed to atmosphere, to allow potential oxidation, and then XPS analysis was performed at Virginia Commonwealth University's Nanomaterials Core Characterization Facility, using a Thermo Scientific ESCALAB 250 Spectrometer. We note that a similar XPS analysis has shown that a $\sim 10 \AA$ thick Al overlayer fully covers a Nb film and prevents oxidation [32]. For this reason, the $\sim 12 \AA$ thick $\mathrm{Al}$ 


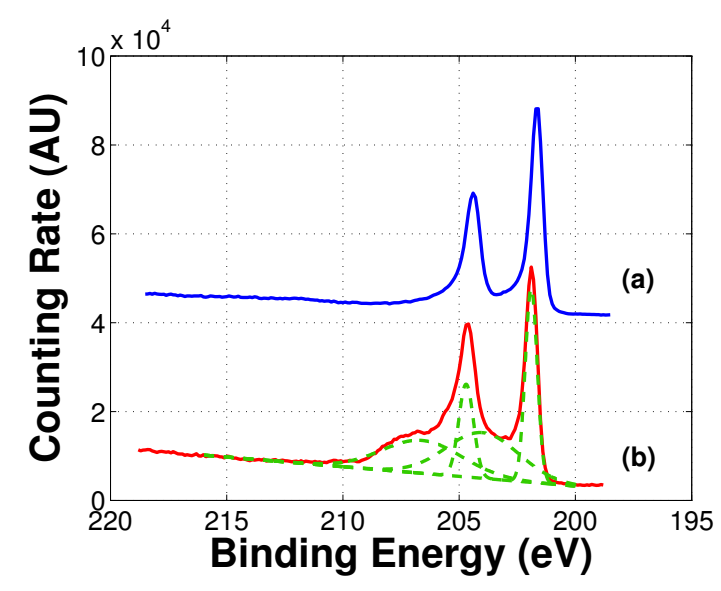

Figure II1: XPS spectrum of Nb film: (a) $12 \AA \mathrm{Al}$ overlayer, (b) $15 \AA$ AlN overlayer and decomposition into four Gaussians (dashed line).

overlayer sample serves as a baseline for comparison.

\section{XPS Analysis of Al and AlN overlayers}

The XPS spectrum of the $2000 \AA \mathrm{Nb}$ film with a $12 \AA \mathrm{Al}$ overlayer is shown in Figure II1a. It contains two distinct peaks located at 201.5 and $204 \mathrm{eV}$. Kwo demonstrated that XPS peaks at $\sim 202$ and $\sim 205 \mathrm{eV}$ correspond to the electron binding energies of pure $\mathrm{Nb}$ while peaks at $\sim 207$ and $\sim 210 \mathrm{eV}$ correspond to the electron binding energies of $\mathrm{Nb}$ bonded with oxygen [32]. It is concluded, from the lack of $\mathrm{Nb}$ oxide peaks in Figure II1a, that the $12 \AA \mathrm{Al}$ overlayer completely covered the underlying $\mathrm{Nb}$ film, preventing the formation of oxides.

Similarly, the XPS spectrum of a $2000 \AA$ Nb film with a $15 \AA$ reactively sputtered AlN overlayer, from our deposition system, is shown in Figure II1b. The spectrum also contains the two distinct $\mathrm{Nb}$ peaks located at 201.5 and $204 \mathrm{eV}$ and lacks distinctive peaks indicative of $\mathrm{Nb}$ oxide formation. The $15 \AA$ reactively sputtered AlN overlayer prevented oxidation of the underlying $\mathrm{Nb}$ film. However, the spectrum is qualitatively different as a small knee is visible in the spectrum from 207-209 eV, indicative of a chemical change in the underlying $\mathrm{Nb}$ unique to reactively sputtered AlN barrier 
growth. The spectrum was decomposed, as shown by the dashed lines in Figure II1 on page 178, using a non-linear least squares curve fit to a sum of four Gaussian functions in Matlab. The results indicate the observed knee may in fact represent a superposition of two separate spectra.

One hypothesis is that there is a Nb-N interaction at the interface, either through reaction with the AlN film or free radicals of $\mathrm{N}$ present during the AlN deposition, as it has been shown NbN based films produce XPS spectra in the range of the two additional peaks shown in the decomposition [98]. From a purely thermodynamic viewpoint, a potential reaction:

$$
\frac{4}{3} \mathrm{Nb}+\frac{4}{3} \mathrm{AlN}+\mathrm{O}_{2} \Longrightarrow \frac{4}{3} \mathrm{NbN}+\frac{2}{3} \mathrm{Al}_{2} \mathrm{O}_{3}
$$

has a free energy of reaction $\triangle \mathrm{G}=-954 \mathrm{~kJ}$ per mole $\mathrm{O}_{2}$, while the energy of formation for $\mathrm{Nb}_{2} \mathrm{O}_{5}$, the most stable native oxide of $\mathrm{Nb}$, is $\triangle \mathrm{G}=-706 \mathrm{~kJ}$ per mole $\mathrm{O}_{2}$. The lack of $\mathrm{Nb}$ oxide peaks in Figure II1b does not preclude incoherent AlN coverage, as in the presence of oxygen, a reaction of type (II.1) is more energetically favorable than the simple oxidation of niobium.

\section{Results and Conclusions}

The $\mathrm{Nb} / \mathrm{AlN} / \mathrm{Nb}$ junctions produced by means of direct deposition of the AlN tunnel barrier on $\mathrm{Nb}$ electrodes through reactive sputtering produced short circuited junctions without SIS IV characteristics. This method has been shown to produce SIS characteristic junctions utilizing $\mathrm{NbN}$ electrodes, however there are no reports in the literature at the time of writing that have demonstrated this method utilizing $\mathrm{Nb}$ electrodes [34]. A possible explanation for this disparity could be due to the different crystal structures of $\mathrm{Nb}$ and $\mathrm{NbN}$. $\mathrm{NbN}$ and $\mathrm{AlN}$ have FCC and $\mathrm{HCP}$ crystal lattices respectively, both are very similar closed-packed lattices with the sole difference in 


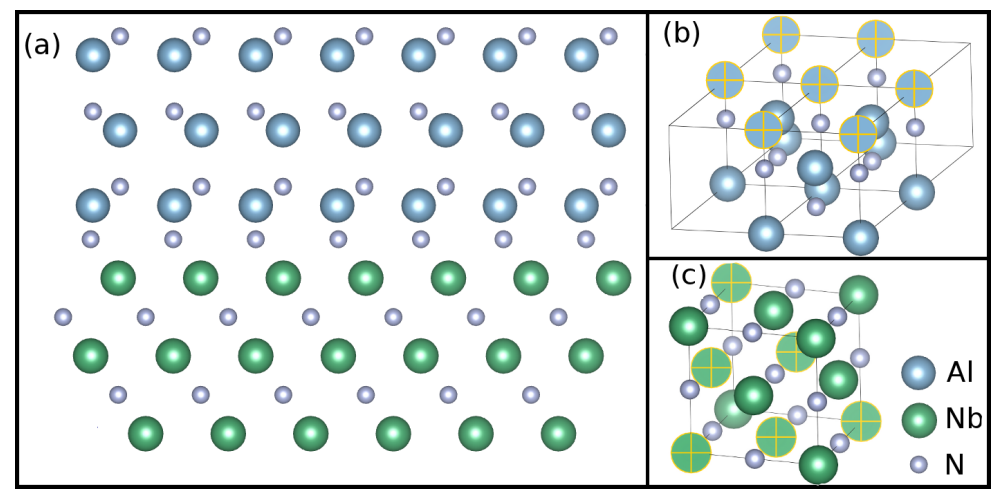

Figure II2: (a) 001 oriented HCP AlN aligned to 111 oriented FCC NbN. View is along 110 direction of NbN. (b) AlN crystal - 001 plane highlighted. (c) NbN crystal - 111 plane highlighted.

the stacking order of close-packed planes. Nb has a non-close-packed BCC lattice which lacks any identical planes with respect to HCP AlN. The close-packed planes of HCP, (001), and FCC, (111), are identical, as shown in Figure II2 and the nearest neighbor separations in these planes for FCC NbN and HCP AlN are $3.15 \AA$ and $3.11 \AA$ respectively [99]. If the near-matching lattice permits local epitaxial growth of AlN on $\mathrm{NbN}$ grains, it could explain why $\mathrm{NbN} / \mathrm{AlN} / \mathrm{NbN}$ tunnel junctions have shown low-leakage IV curves in the literature. We note that reactively sputtered NbTiN has a FCC lattice with a lattice constant of 4.24 to $4.38 \AA$, which produces a mere 1-3\% lattice mismatch with AlN [100].

With this in mind, an additional trilayer sample was produced, but NbTiN (owing to its lattice match with AlN) was used for both the base and counter electrode. NbTiN films were deposited by means of reactively sputtering from a NbTi target (4.0 mT, 1.0 A, $\Delta \mathrm{V}=20 \mathrm{~V})$, the details of which are described elsewhere [24]. POGO test chips were fabricated and tested using the Al Quadlevel process and new test mask described in Chapter 4. The resulting IV characteristics are shown in Figure II3 - the characteristics were similar across all tested junctions. We note a very high subgap leakage, but some signs of SIS behavior are observed, in contrast to the case of our sample using $\mathrm{Nb}$ base electrodes - this argues that crystal structure of the 


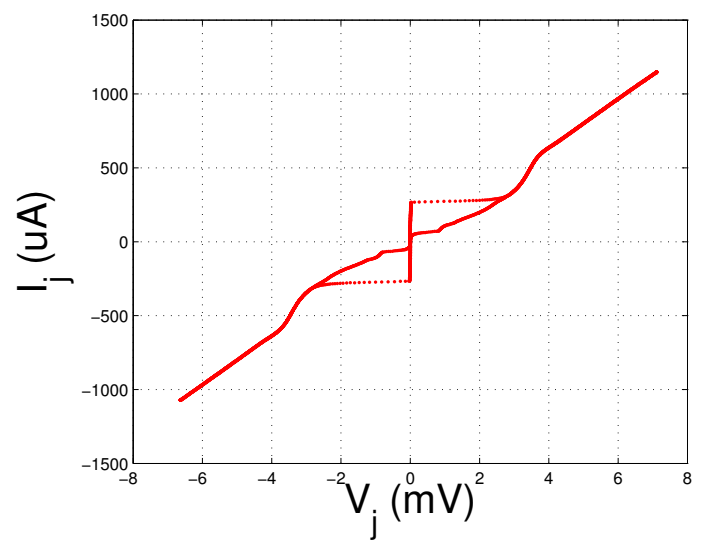

Figure II3: Typical IV characteristics of a NbTiN/AlN(Sputtered)/NbTiN SIS junction $\left(J_{C} \approx 13 \mathrm{kA} / \mathrm{cm}^{2}\right)$.

base electrode plays a role in direct barrier deposition.

While the IV characteristics shown in Figure II3 are poor, and not usable for heterodyne mixing, we note this was a first attempt at creating an all NbTiN based SIS junction. Such a device has not been realized at the time of writing, but the signs of SIS behavior observed in this initial study are promising. It is plausible that further research to optimize the deposition of the NbTiN and AlN films, may increase the quality of the resulting junction. We also note that the NbTiN films used in this study were deposited it at room temperature - it is also plausible that the increased crystallinity achieved through heated deposition of NbTiN films, a process recently available in our research laboratory, may provide a better surface for epitaxial AlN growth. For future work, X-ray diffraction (XRD) will be used to report on the crystal structure of our NbTiN and AlN films and potentially investigate the variation of lattice constants through deposition parameters. We also note, the work presented in this dissertation lays the ground work to better investigate new trilayer structures. The Al Quadlevel process and new test mask set presented in Chapter 4 allows for rapid fabrication of SIS test junctions. Additionally, the ellipsometric models, developed as part of the work in chapter 5, lay the groundwork to monitor the deposition rates of directly deposited AlN films in order to produce trilayer of 
reasonable $R_{N} A$ values for testing. We also note, we can in situ determine the optical constants of an AlN film through our ellipsometric models. This opens the possibility to tailor deposition conditions such that the optical constants better match ideal AlN values. 


\section{Appendix III}

\section{Modifications to Trilayer}

\section{Deposition System}

Included in this appendix is a summary of the modifications performed on the Trilayer Deposition tool as described in Chapter 3, as well as a planned modification to the nitridation chamber. All vacuum chamber extensions and couplings were manufactured by Kurt J. Lesker Company®, and the final approved CAD drawings are included with permission.

\section{Original Tri-3 Deposition Tool}

The original configuration of Tri-3 before modifications is shown in Figure III1. The tool consists of two chambers: (1) the deposition chamber which contains the 4 DC magnetron sputter guns, and (2) the nitridation chamber that contains the ICP unit used for both in situ cleaning of the samples and nitridation of Al overlayers. The nitridation chamber consists of 3 main parts: (1) the load lock where samples are introduced to the chamber, (2) the DN160 cube, an alternate loading area to quickly access the ICP and ellipsometer, and (3) the ICP tube that contains the sample stage the wafer sits atop for access to the ICP and ellipsometer. In the original 
design, the entire nitridation chamber is vented, leading to frequent introduction of contamination of the ICP tube. The implemented modifications are described below.

\section{Addition of Turbo Molecular Pump to DN160 Cube}

A Pfeiffer Vacuum HiPace ${ }^{\circledR} 700$ turbo pump was added to the DN160 cube to increase the pumping efficiency of the nitridation chamber. This was accomplished by the addition of a 6.5" long DN160 tube extension, with 2 DN40 conflat accessory ports to accommodate both an RGA and ION gauge, and 1 DN16 conflat accessory port to accommodate a capacitance manometer. Between the turbo pump and tube extension, a VAT® Series 64.2 Control Gate Valve with stepper motor and PM5 controller was installed. The controllable gate valve allows for accurate pressure control in the nitridation chamber. The CAD drawing of the tube extension is included on the following page. 


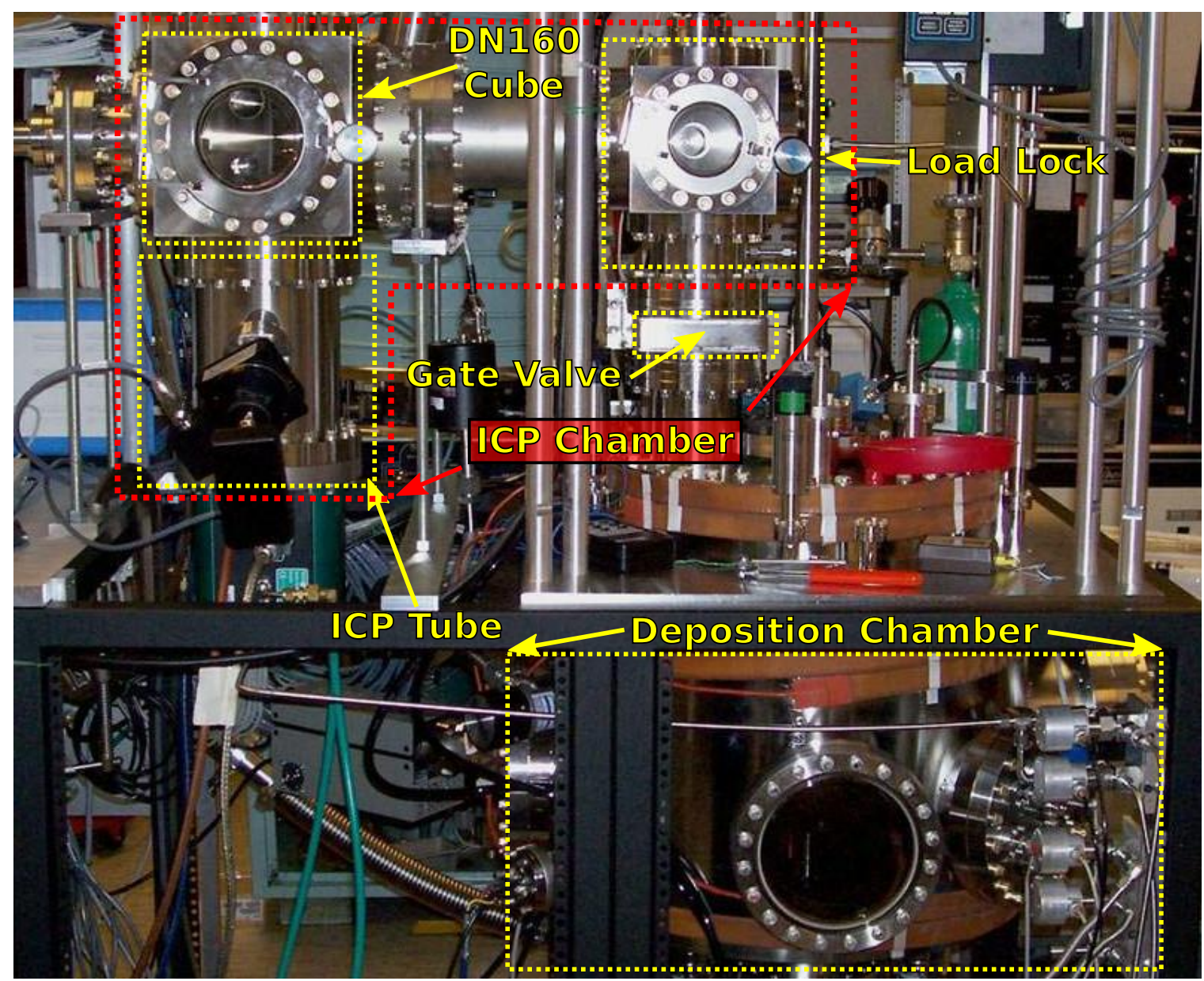

Figure III1: The original Tri-3 tool, as described in the work by Cecil [39]. The system consists of 2 isolated chambers: (1) the deposition chamber that is held under high vacuum, and (2) the nitridation chamber (consisting of the ICP tube, DN160 cube, and load lock) that is vented while loading a sample. 


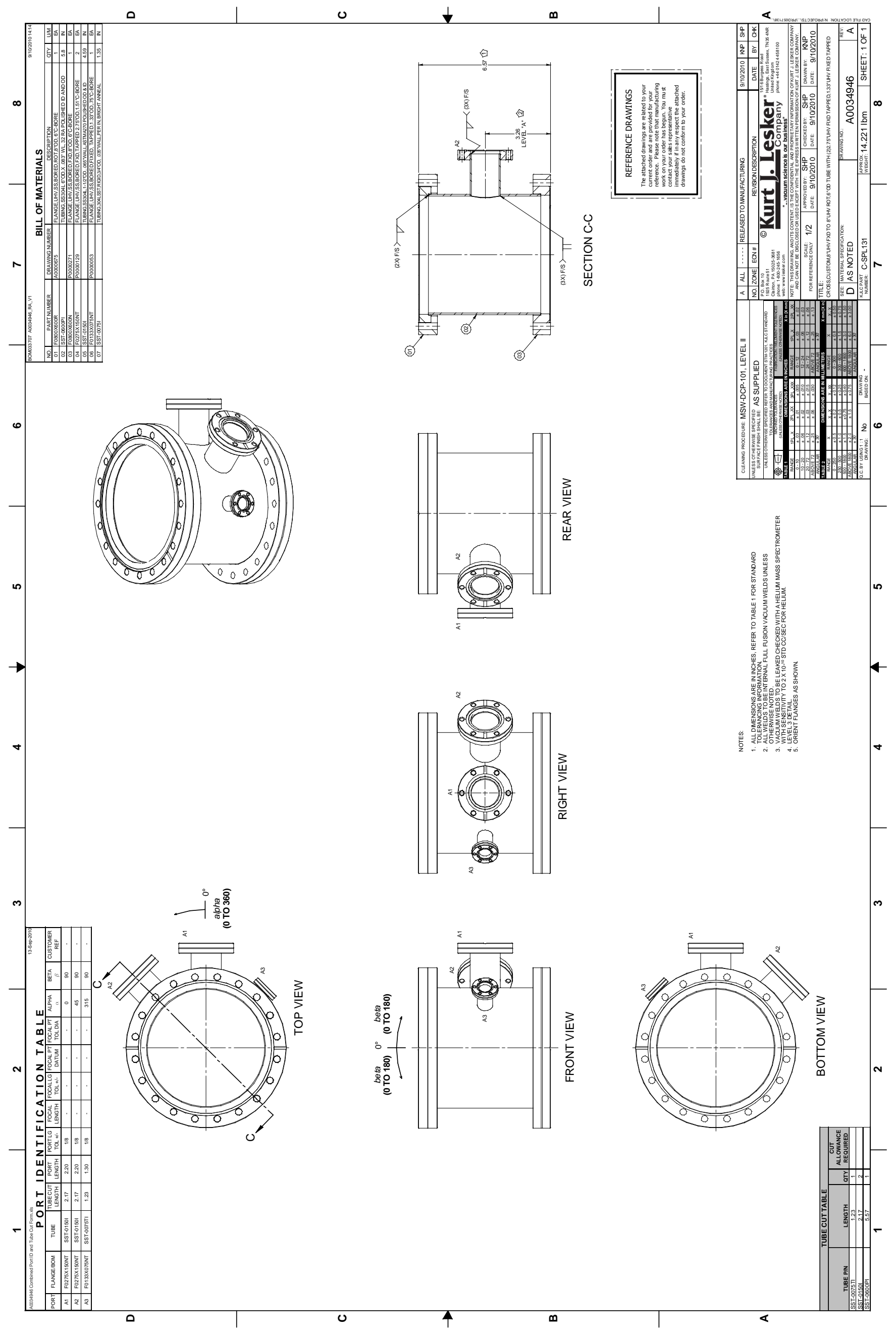




\section{Addition of Turbo Molecular Pump to Load Lock}

\section{Cube}

Similarly, a Pfeiffer Vacuum HiPace® 300 turbo pump was added to the load lock cube to increase the pumping efficiency. This was accomplished by the addition of a 5.8" long DN100 tube extension, with 3 DN40 conflat accessory ports to accommodate a capacitance manometer, TELEVAC CC-10 wide range vacuum gauge, and gas feed through (for introducing oxygen for $\mathrm{Al}_{2} \mathrm{O}_{3}$ formation). Between the turbo pump and tube extension, a Kurt J. Lesker® SG0400PVCF pneumatic gave valve was installed. The CAD drawing of the tube extension is included on the following page. 


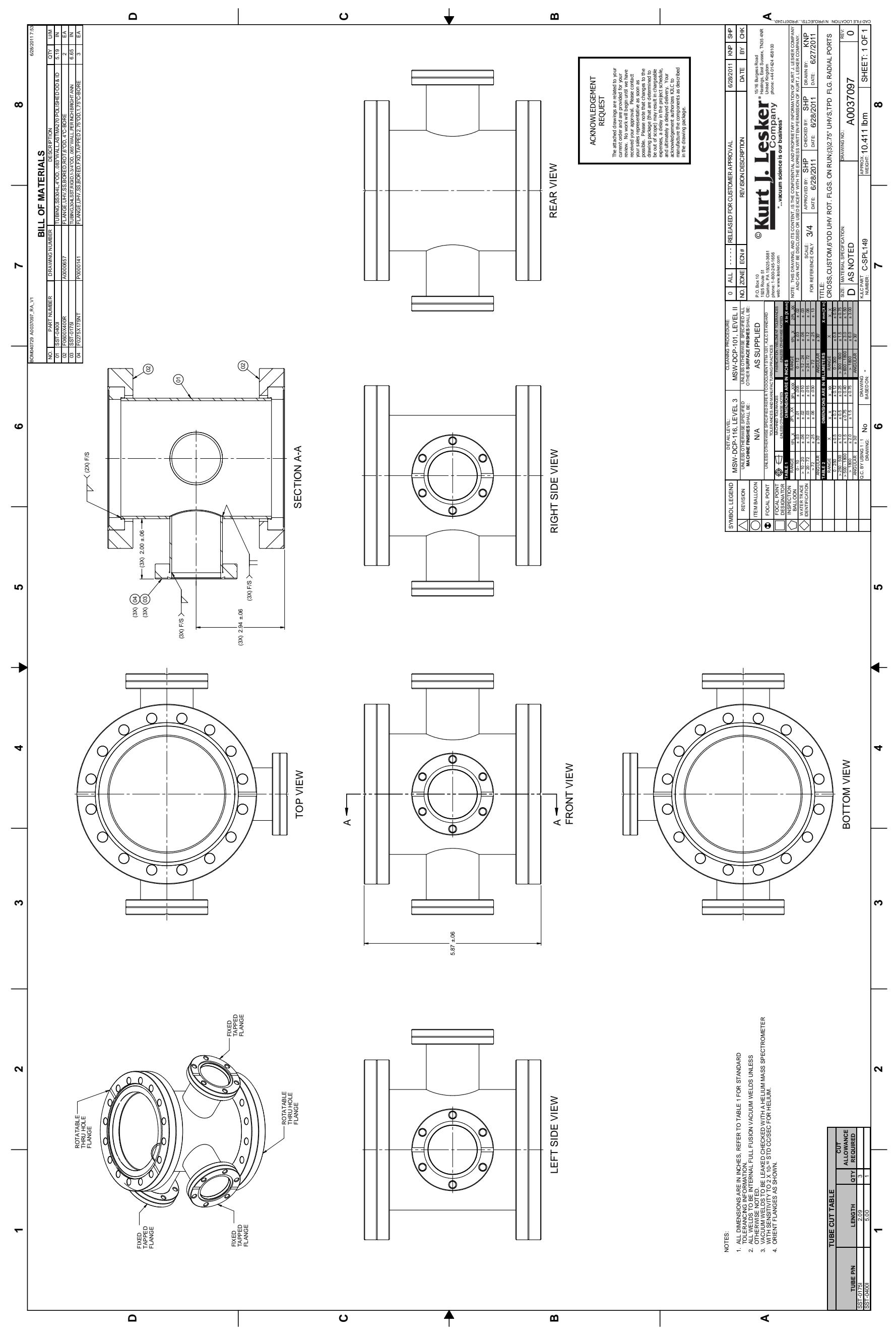




\section{Addition of ION Gun and Load Lock isolation}

As mentioned in Chapter 3, the original design of Tri-3 used a high power Ar plasma to in situ clean our samples by means of etching the sample surface. To further help eliminate the possibility of contamination due to in situ cleaning, an MPS 3000 ion gun ion gun was installed between the load-lock and nitridation chamber to allow in situ cleaning of our samples away from critical components and dedicate the ICP to nitrogen plasma chemistries needed for AlN growth. Additionally, a Kurt J. Lesker® SG0400PVCF pneumatic gave valve was installed to isolate the load lock from the ICP tube. The result of these modifications are 3 isolated chambers: (1) the deposition chamber, (2) nitridation chamber containing the ICP unit, ICP tube, ion gun, and DN 160 cube, and (3) a dedicated load lock. All three chambers are pumped independently by turbo pumps. To accommodate the ion gun, a custom 6" long 4" OD tube extension, with both DN160 and DN100 conflat flanges on either end was designed. The tube extension features a DN75 port to accommodate the ion gun, as well as a DN40 port opposite the DN75 port to accommodate a sight glass to aid in centering the sample above the ion gun during in situ cleaning. The gate valve was attached to the DN100 conflat port of the tube extension. To mate the gate valve to the load lock cube, we note only $2.1 "$ of lateral clearance was available. Noting this, a custom 2.1" DN100 tube was manufactured with rotatable flanges. As shown in Figure III2, rotatable flanges were required, as well as custom machined studs and screws to fit in the tight clearance. All resulting modifications to the Tri-3 are shown in Figure III2, and the CAD drawings for the ION gun tube, and 2.1" DN100 tube are included in the following pages. 

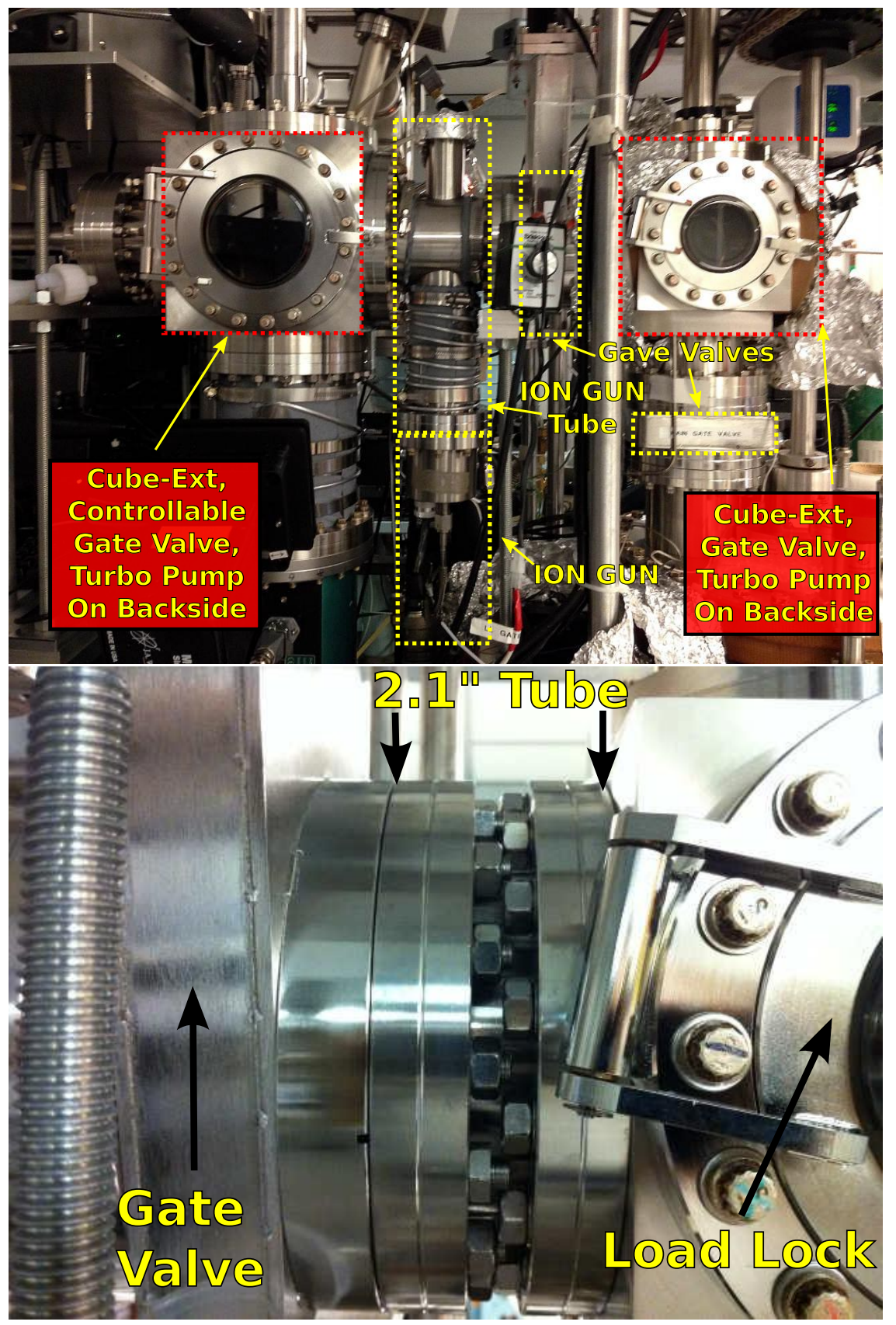

Figure III2: Top: Tri-3 after modifications. Bottom: 2.1" extension tube that couples the load lock and nitridation chamber. 


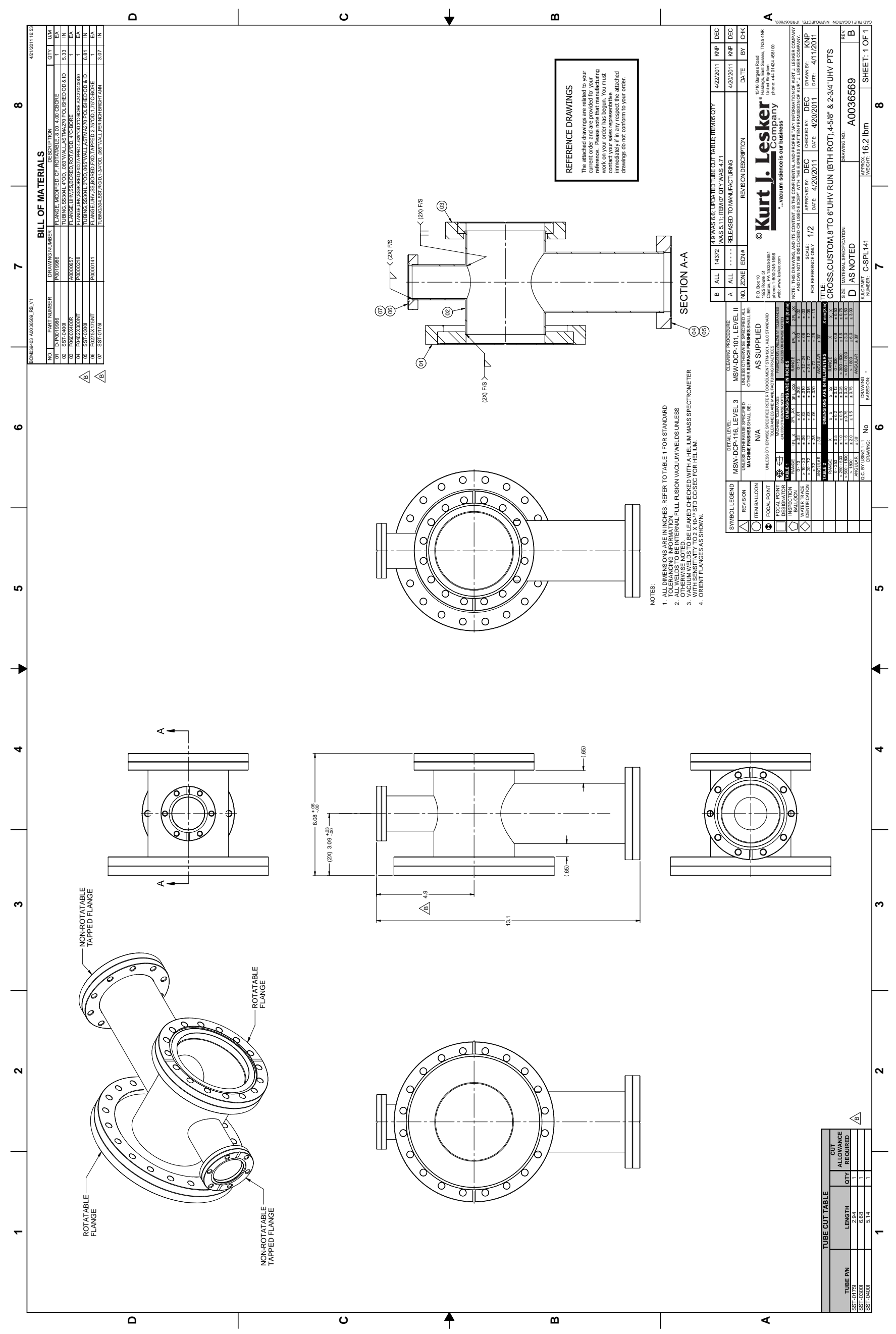




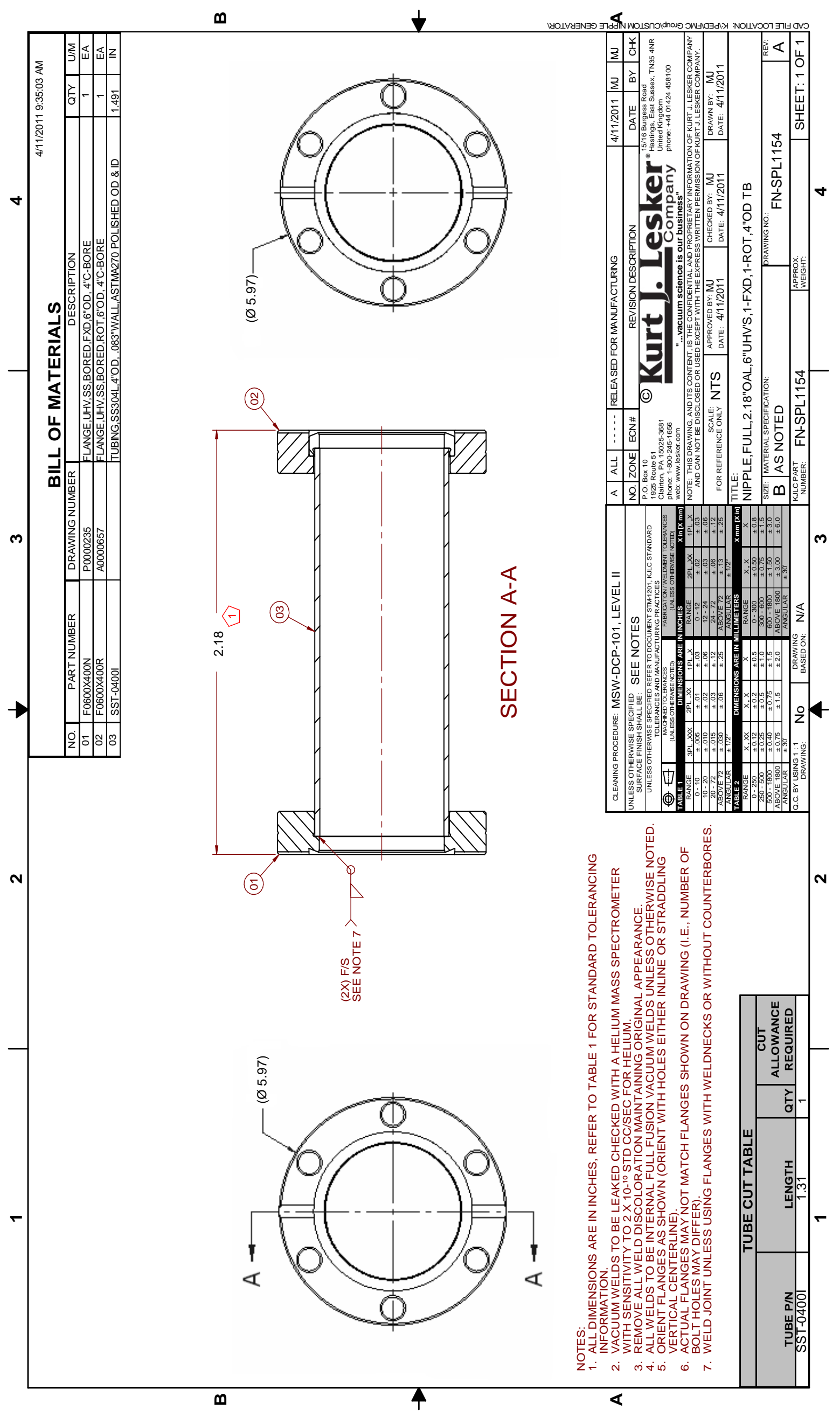




\section{ICP Tube Extension}

Noting the results from Chapter 6, it is argued that increasing the ICP source to sample separation will reduce the AlN growth rate during ICP nitridation of $\mathrm{Al}$ overlayers when high dissociation plasmas are used. Noting this, a 5" DN160 tube extension, to fit between the ICP unit and existing ICP tube was designed. The extension also features a DN40 port to accommodate a quartz view port. The view port will allow spectroscopic observation of the ICP using the methods described in Chapter 5. The CAD drawing of the extension is included on the following page. 


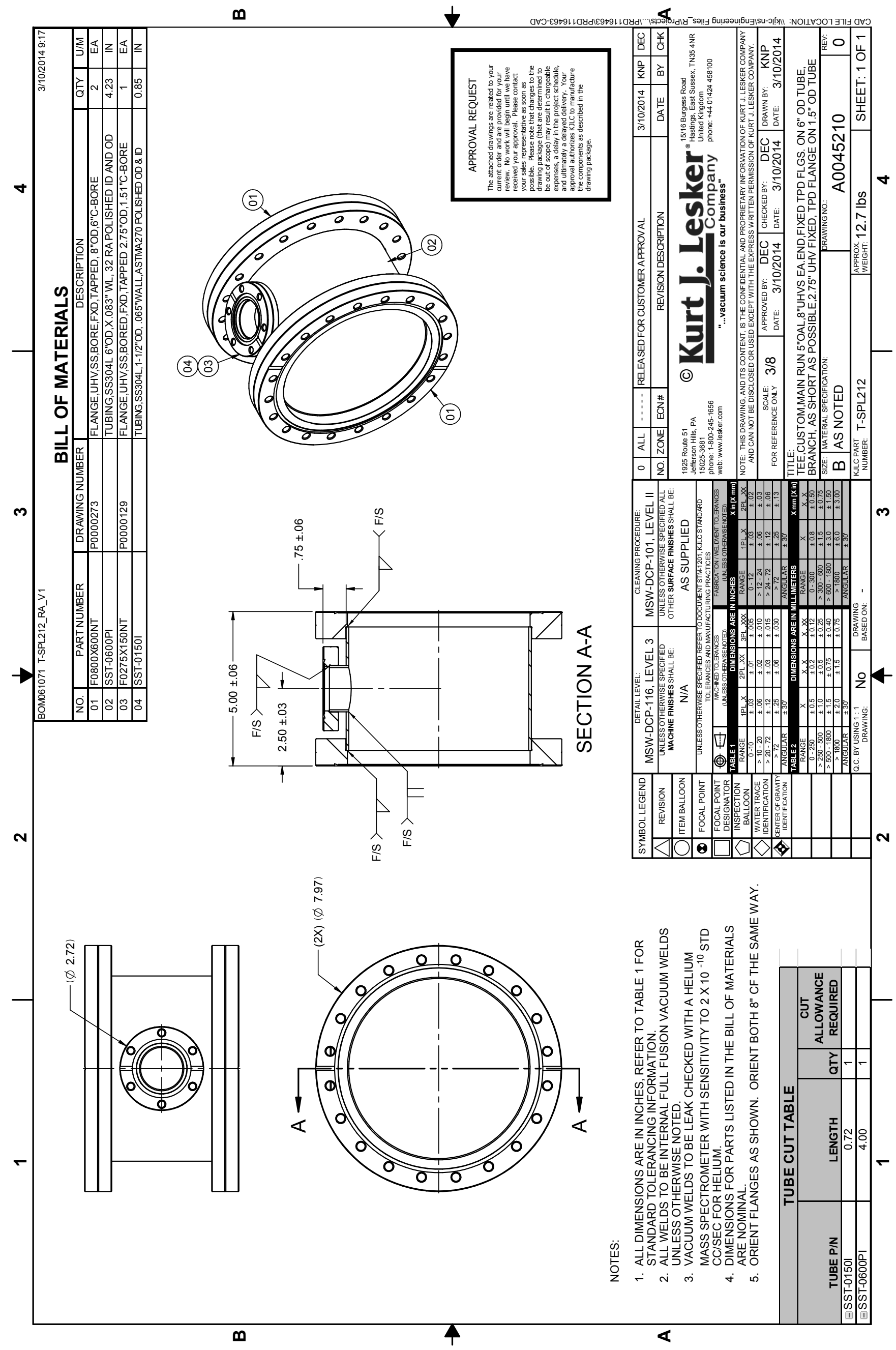


Appendix IV

Al Quadlevel Process Sheets and

New Test Mask Info 


\section{Test-Mask Process Sheets \\ Trilayer With Quadlevel AI}

\section{Base Patterning}

$\square$ Base Lithography

$\square$ Trilayer Etch

$\square$ Resist strip

$\square$ Quadlevel

$\square$ NFR/Poly/Al Definition

$\square \mathrm{SiO2}$ Deposition/liftoff

$\square$ m3/wiring Step

$\square$ m3/Au Wire Lithography

$\square$ m3/Au Deposition

$\square$ m3/Au Liftoff

$\square$ Dice 


\section{Trilayer Base Lithography}

(1) ETH, TCE, METH spin clean

(2) (2) MARCH O2 100W > 20minutes

(3) $110^{\circ} \mathrm{C} \mathrm{HPB}$ for 5 minutes

(4) HMDS 5minutes

(5) Spin AZ5209 at $4 \mathrm{kRPM}$ for $30 \mathrm{~s}$

(6) Pause $5 \mathrm{~min}$

(7) $100^{\circ} \mathrm{C}$ HPB for $90 \mathrm{~s}$

(8) a) If using MJB4: Expose 10.5s on with Base mask $\mathrm{LI}=$ , $\mathrm{LP}=$ $\left(10.7 \mathrm{~s} *\left(7 \mathrm{~mW} / \mathrm{cm}^{2}\right)=75 \mathrm{~mW}-\mathrm{s} / \mathrm{cm}^{2}\right)$

b) If using EVG: No Filter. Exposure= $\mathrm{s}(\sim 15 \mathrm{~s}) . \mathrm{H} / \mathrm{C}$ press $=$ psi ;

Chuck Press $=$ bar

(9) Pause 5 min

(10) Develop 400K (1:4) $\mathrm{t}=$ $\min [$ clear $+20 \mathrm{~s}(\sim 50 \mathrm{~s})]$

(11) 3 DI rinses

(12) Inspect, COMMENTS

(13) Thickness profile

\section{EPROM - Bake}

DATE Operator $=$

(1) $110^{\circ} \mathrm{C}$ HPB $1 \mathrm{~min}$

(2) EPROM $30 \mathrm{~min}$

(3) $160^{\circ} \mathrm{C}$ HPB 2 min

\section{Au Wet Etch}

(1) $130 \mathrm{hpb} 10 \mathrm{~min}$ 2 minute pause, then into

(2) MARCH O2 $120 \mathrm{~W} 2 \mathrm{~min}$

(3) right into HG800 25 seconds

(4) 4 DI rinse (5) Blow thoroughly

(5) Inspect resist 


\section{Oxford Trilayer etch}

(1) Heat beaker of DI water to 90C, you MUST dip wafer directly into water after etch.

(2) Nb Conditioning: Load Recipe: "Trilayer w/Au Cond BCl3 + Cl2 => SF6+Ar".

(3) Recipe Parameters: 2 steps:

(1) $\mathrm{O} 2$ clean: $\mathrm{O} 2=20 \mathrm{sccm}, \mathrm{RF}=100 \mathrm{~W}$, Press $=20 \mathrm{mT}, \mathrm{T}=10 \mathrm{C}, \mathrm{He}=5 \mathrm{~T}$, Time $=10 \mathrm{~min}$.

(2) $\mathrm{SF} 6=20 \mathrm{sccm}, \mathrm{Ar}=10 \mathrm{sccm}, \mathrm{RF}=30 \mathrm{~W}$, Press $=10 \mathrm{mT}, \mathrm{T}=10 \mathrm{C}, \mathrm{He}=5 \mathrm{~T}$, Time $=2: 00 \mathrm{~min}$.

(3) $\mathrm{BCl} 3=40 \mathrm{sccm}, \mathrm{Cl} 2=9 \mathrm{sccm}, \mathrm{RF}=80 \mathrm{~W}$, Press $=50 \mathrm{mT}, \mathrm{T}=10 \mathrm{C}, \mathrm{He}=5 \mathrm{~T}$, Time $=3 \mathrm{~min}$. Run conditioning step.

Voltage

Ref Pwr

He Flow

Conditioning run complete, platter returns to LL. vent L.L.

(4) 120C HPB Wafer (for Nb etch) for $2 \mathrm{~min}$

(5) Load sample on center of $\mathrm{Si} / \mathrm{SiO} 2$ platter in LL.

Pump in service mode, use soft pump valve on load lock to prevent wafer from moving

(6) Turn Endpoint Camera on, remove cap

(7) Load Recipe: "Trilayer BCl3+Cl2 SF6 => Ar".

Recipe Parameters:

(1) $\mathrm{Ar}=50 \mathrm{sccm}, \mathrm{SF} 6=00 \mathrm{sccm}, \mathrm{RF}=140 \mathrm{~W}$, Press $=20 \mathrm{mT}, \mathrm{T}=10 \mathrm{C}, \mathrm{He}=5 \mathrm{~T},(5 \mathrm{~min})$

(2) $\mathrm{BCl} 3=40 \mathrm{sccm}, \mathrm{Cl} 2=9 \mathrm{sccm}, \mathrm{RF}=80 \mathrm{~W}$, Press $=50 \mathrm{mT}, \mathrm{T}=10 \mathrm{C}, \mathrm{He}=5 \mathrm{~T}$

(3) $\mathrm{SF} 6=20 \mathrm{sccm}, \mathrm{Ar}=10 \mathrm{sccm}, \mathrm{RF}=30 \mathrm{~W}$, Press $=10 \mathrm{mT}, \mathrm{T}=10 \mathrm{C}, \mathrm{He}=5 \mathrm{~T}$

Recipe set time is longer than necessary so etch can be stopped manually.

(8) Run recipe... Wafer will load ... position and focus endpoint camera.

(9) Watch endpoint \& wafer and evaluate: Terminate etch time appropriately

Comments:

Etch time $=$ Voltage He Flow

(10) Vent L.L. remove wafer and place in beaker of warm DI water $>5$ minutes.

(11) Microscope Inspect: 


\section{AZ5209 Resist Stripping}

NOTE: You MUST use heated NMP+P.Glycol to remove the chlorinated resist, if you fail to do this step, the wafer will remain covered in debris which is very difficult to remove.

(1) March 120W $10 \mathrm{~min}$

(2) $\quad 110^{\circ} \mathrm{C} \mathrm{NMP} \mathrm{+} \mathrm{P.} \mathrm{Glycol[1:1]} \mathrm{stripping} 60 \mathrm{~min}$

(3) Lightly swap with E-Glycol

(4) Back in NMP for $30 \mathrm{~min}$

(5) $4 \times$ DI rinse $=>$ Microscope inspect

(6) MARCH $100 \mathrm{~W}, 30 \mathrm{~min}$

(7) Inspect:

(8) Thickness Profile: (Trilayer + Qz etched):

$\AA$.

(9) Mandatory SEM:

(10) Inspect 


\section{NFR/Polyimide}

\section{NO TCE ON AIN Underlayer wafers!}

(1) ETH, METH spin clean wafers,

(2) Ozone $5+\min$

(3) $110^{\circ} \mathrm{C} \mathrm{HPB}$ for $\geq 5 \mathrm{~min}$ $160^{\circ} \mathrm{C} \mathrm{HPB}$ for $1 \mathrm{~min}$

(4) Let cool 2 min

(5) Spin NFR:M2P [1:2] as adhesion layer $6 \mathrm{kRPM}, 30 \mathrm{~s}$ with $0.2 \mathrm{um}$ filter

(6A) $100^{\circ} \mathrm{C} \mathrm{HPB}$ $2 \mathrm{~min}$ [to set]

(6B) $160^{\circ} \mathrm{C}$ HPB 5 min [to cure]

(7) EPROM $30 \mathrm{~min}$

(8) $\mathbf{1 3 0}^{\circ} \mathrm{C}$ HPB $5 \mathrm{~min}$ , Pause 1minute then

(9) Spin OCG Durimide (TM) 284:Thinner [3:1] $4 \mathrm{kRPM}, 30 \mathrm{~s}$ (No primer, no filter) (this is what we use for trilevel, so it's a bit thicker.. think its around 3,800ang )

(10) $130^{\circ} \mathrm{C}$ HPB $2 \min$ [to set]

(11) $160^{\circ} \mathrm{C} \mathrm{HPB}$ $10 \min$ [to-cure]

(11) INSPECT, Comments

\section{Trilevel Al Deposition ( 1000Ang)}

Fill in conditions, Currently 20 minutes in Diode Sputter@10 mT and 600V.

\section{Junction Photolithography}

(1) Spin clean Eth, Meth only NO TCE

(2) Ozone $5 \mathrm{~min}$

(3) $110^{\circ} \mathrm{C}$ HPB for $5 \mathrm{~min}$

(4) Let cool $2 \mathrm{~min}$

(5) Spin HMDS for a few seconds

(6) $\quad 110 \mathrm{C} 30 \mathrm{~s}$

(7) Spin NFR:M2P [3:1 by mass] 6 kRPM [0.2-filtered]

(8) $100^{\circ} \mathrm{C} \mathrm{HPB} 120 \mathrm{~s}$

(9) Load to the EVG___ ALIGN WAFER

(10) Expose ___ $\mathrm{s}(\sim 35 \mathrm{~s})$ on EVG With Mesh filter

(11) $100 \mathrm{PEB} 60 \mathrm{~s}$

(12) $\mathbf{3 0 0}$ MIF develop for $\mathrm{s}(30 \mathrm{~s})$ 


\section{Al Wet Etch}

Heat up Transene before you begin, and make sure stir bar is around $300 \mathrm{rpm}$.

Pause Stir bar right before wet etch.

(1) March 100W 2 minutes

(2) Pause Stir bar right before wet etch.

(3) Right into Transene type-D @ 50 C Actual Temperature:

(4) Etch $+5-6$ s over, $t=$ $\mathrm{s}(\sim 60 \mathrm{~s})$

(5) Mandatory SEM:

\section{Oxford RIE "Polyimide" (ie, organic) Etch}

1. Conditioning: Load Recipe: “Poly Condition -50C 8W 5E/2P Lower Power".

Recipe Parameters: 2 steps:

(1) $\mathrm{O} 2=15 \mathrm{sccm}, \mathrm{RF}=100 \mathrm{~W}$, Press $=10 \mathrm{mT}, \mathrm{T}=-50 \mathrm{C}, \mathrm{He}=5 \mathrm{~T}, \mathrm{t}=5 \mathrm{~min}$.

(2) $\mathrm{O} 2=15 \mathrm{sccm}, \mathrm{RF}=8 \mathrm{~W}$, Press $=10 \mathrm{mT}, \mathrm{T}=-50 \mathrm{C}, \mathrm{He}=5 \mathrm{~T}, \mathrm{t}=5 \mathrm{~min}$.

2. Run condition recipe

Voltage (100V) Ref Pwr

He flow

3. Conditioning Run complete, wafer returns to LL, Vent LL

8. HPB Sample (for 'poly' etch) for $2 \mathrm{~min}$. at 120C.

9. Load sample on center of platter in LL.

Pump in service mode, use soft pump valve on load lock to prevent wafer from moving

10. Poly etch : Load Recipe: "Poly Etch -50C 8W 5E/2P 24cycle".

Recipe Parameters: 2 steps:

(1) 2 minute pause: $\mathrm{O} 2=15 \mathrm{sccm}$, Press $=10 \mathrm{mT}, \mathrm{T}=-50 \mathrm{C}, \mathrm{He}=5 \mathrm{~T}$

(2) 5 minute etch: $\mathrm{O} 2=15 \mathrm{sccm}, \mathrm{RF}=8 \mathrm{~W}$, Press $=10 \mathrm{mT}, \mathrm{T}=-50 \mathrm{C}, \mathrm{He}=5 \mathrm{~T}$

Twenty-four cycles for etching nfr(1:2)/poly(3:1)@6kRPM

11. Run etch recipe for (24) Repeat cycles ( $3 \mathrm{hrs})$ \{this for (nfr 1:2)/(284poly3:1)\}

Voltage (100V) Ref Pwr He flow

12. Run complete, wafer will return to LL.

13. Vent LL, remove wafer.

14. Inspect. Comments? 
15. Thickness profile. Thickness

16. Mandatory SEM (profile, measure top and bottom features... is the surface clean, free of debris, free of grass?)

17. Comments (profile) 


\section{Test Mask M2 Nb RIE}

Sample A: Power: $\square$ 30W or $\square$ 50W / Grease: $\square$ No-Grease $\square$ W/L-Grease Sample B: Power: $\square$ 30W or $\square$ 50W / Grease: $\square$ No-Grease $\square$ W/L-Grease

Sample C: Power: $\square$ 30W or $\square$ 50W / Grease: $\square$ No-Grease $\square$ W/L-Grease Sample D: Power: $\square$ 30W or $\square$ 50W / Grease: $\square$ No-Grease $\square$ W/L-Grease Oxford RIE $\mathrm{Nb}$ (m2) etch

(1) Nb Conditioning: Load Recipe: "Nb XXW etch-condition Si/SiO2 10Deg".

(2) Recipe Parameters: 2 steps:

(1) $\mathrm{O} 2$ clean: $\mathrm{O} 2=15 \mathrm{sccm}, \mathrm{RF}=100 \mathrm{~W}$, Press $=20 \mathrm{mT}, \mathrm{T}=10 \mathrm{C}, \mathrm{He}=5 \mathrm{~T}$, Time $=6 \mathrm{~min}$.

(2) $\mathrm{Ar}=10 \mathrm{sccm}, \mathrm{SF} 6=20 \mathrm{sccm}, \mathrm{RF}=\mathrm{XXW}$, Press $=10 \mathrm{mT}, \mathrm{T}=10 \mathrm{C}, \mathrm{He}=5 \mathrm{~T}$, Time $=4 \mathrm{~min}$.

$\approx$ Run conditioning step.

Voltage A

B: $\quad C$

D:

He Flow A:

B:

C:

D:

(3) 110C HPB Wafer (for $\mathrm{Nb}$ etch) for $2 \mathrm{~min}$.

(4) Load sample on center of $\mathrm{Si} / \mathrm{SiO} 2$ platter in LL.

Pump in service mode, use soft pump valve on load lock to prevent wafer from moving

(5) Turn Endpoint Camera on, remove cap

(6) Load Recipe: "Nb + Au XXW etch 5 min 10Deg".

Recipe Parameters: $\quad A r=50 \mathrm{sccm}, S F 6=00 \mathrm{sccm}, R F=140 \mathrm{~W}$, Press $=20 \mathrm{mT}, \mathrm{T}=10 \mathrm{C}, \mathrm{He}=5 \mathrm{~T}$,

$\mathrm{Ar}=10 \mathrm{sccm}, \mathrm{SF} 6=20 \mathrm{sccm}, \mathrm{RF}=\mathrm{XXW}$, Press $=10 \mathrm{mT}, \mathrm{T}=10 \mathrm{C}, \mathrm{He}=5 \mathrm{~T}$,

\section{In-situ Au Etch}

(7) Sample A: Etch time =

Voltage

He Flow

Date:

Sample B: Etch time $=$ Voltage He Flow Date:

Sample C: Etch time $=$ Voltage He Flow Date:

Sample D: Etch time = Voltage He Flow Date:

Nb Etch

(8) Sample A: Etch time $=$ Overetch $=$ Voltage He Flow

Sample B: Etch time = Overetch $=$ Voltage He Flow

Sample C: Etch time $=$ Overetch $=$ Voltage He Flow

Sample D: Etch time = Overetch $=$ Voltage He Flow

(9) Comments/Endpoint sketch:

(10) Inspect:

(11) Mandatory SEM: (surface cleanliness, etched profile, junction size?) 


\section{Test Mask SiO2 Sputtering}

\section{Sample A:}

\section{Sample B:}

\section{Sample C:}

\section{Sample D:}

\section{Test Mask Liftoff}

(1) Inspect w/microscope, COMMENTS

(2) Heat NMP:P-glycol set-at $120 \mathrm{C}$ on hot plate with stir bar with [coverglass]

(3) $\quad\left(\right.$ when $\left.\mathrm{T}=120^{\circ} \mathrm{C}\right) \mathbf{A}$ :

B:

C:

D:

(4) Load wafer in basket (pattern upside down)

(5) Soak $\mathrm{t} \geq(60 \mathrm{~min}) \mathrm{A}$ :

B:

C:

D:

(6) after 40 min start up new NMP

(7) remove basket/wafer, cool $5 \mathrm{~s}$, place on several square Crwipes

(8) Gently qtip with lots of [cold] Ethylene glycol (after first 60min soak) from inside to out. Don't forget the wafer edge liftoff debris seems to amass there, change qtips as needed, liberal use of Eth Glycol

(9) back in old NMP for 5 min, then into new (hot) NMP

(10) Soak $\mathrm{t}=(60 \mathrm{~min})$ (in the new hot NMP) $\mathbf{A}$ :

B:

C:

D:

(11) gently qtip with lots of [cold] Ethylene glycol, don't forget the wafer edge/change qtips

(12) 5 min hot NMP; Final Eth Glycol swab

(13) 4 DI THOROUGHLY

(14) inspect, more lift if needed - DI as above.

(15) Do not march until you are finished with liftoff. The NFR will protect underlying Au

(16) MARCH $100 \mathrm{~W}, \geq 30 \mathrm{~min} \mathbf{A}$ :

B:

C:

D:

(17) Thickness profile:A:

B:

C:

D:

(18) Also across $\mathrm{Q} / \mathrm{SiO}$ edge on wafer $\mathbf{A}$ :

B: C: D: 


\section{m3/Au Wire Lithography - Negative Resist}

You must use NFR for adhesion! Then AZ 5214.

\section{(Use Mask for Trilayer without Au)}

(1) ETH,D-Lim, METH spin clean

(2) MARCH O2 $100 \mathrm{~W}>20$ minutes

(3) $110^{\circ} \mathrm{C}$ HPB for 5 minutes

(4) HMDS 5minutes

(5) Spin NFR+M2P [1:2\} at $6 \mathrm{kRPM}$ for $30 \mathrm{~s}$

(6) $110^{\circ} \mathrm{C} \mathrm{HPB} 60 \mathrm{~s}$

(7) Spin AZ5214 at $4 \mathrm{kRPM}$ for $30 \mathrm{~s}$

(8) $100^{\circ} \mathrm{C}$ HPB for $120 \mathrm{~s}$

(9) MJB4: Exposure $=(62 \mathrm{~s})$ A: B: C: D: Hard Contact only to prevent resist sticking to mask

(10) $100^{\circ} \mathrm{C}$ HPB for $90 \mathrm{~s}-$ for image reversal

(11) Flood expose MJB3/4 for $45 \mathrm{~s}$

(12) Develop 300 MIF [clear+10s ( 20s)] A:

B: C: D D :

(13) 3 DI rinses

(14) Inspect, COMMENTS

(15) Thickness profile

A:

B:

C:

D: 


\section{M3/Au Deposition}

\section{/ m3(Nb)/Au Deposition}

Sample A:

MARCH 100W, 5min . $\mathrm{HPB} 110^{\circ} \mathrm{C} 2 \mathrm{~min}$

Run\# System

Nb Thickness Au Thickness

Sample B:

MARCH 100W, 5min . HPB $110^{\circ} \mathrm{C} 2 \mathrm{~min}$

Run\# System

Nb Thickness Au Thickness

Sample C:

MARCH 100W, 5min . HPB $110^{\circ} \mathrm{C} 2 \mathrm{~min}$

Run\# System

Nb Thickness Au Thickness

Sample D:

MARCH 100W, 5min . HPB $110^{\circ} \mathrm{C} 2 \mathrm{~min}$

Run\# System

Nb Thickness Au Thickness 


\section{NFR/AZ5214 M3/Au liftoff}

\section{Sample A:}

(1) $110^{\circ} \mathrm{C} \mathrm{NMP} \mathrm{+} \mathrm{P.} \mathrm{Glycol[1:1]} \mathrm{stripping} 60 \mathrm{~min}$

(2) Lots of Cold E. Glycol clean room swabs - center to perimeter

(3) Back in $110^{\circ} \mathrm{C} \mathrm{NMP}+\mathrm{P}$. Glycol stripping $60 \mathrm{~min}$

(4) 4 x DI rinse

(5) MARCH $100 \mathrm{~W}, 30 \mathrm{~min}$

(6) Inspect:

\section{Sample B:}

(7) $110^{\circ} \mathrm{C} \mathrm{NMP}+\mathrm{P}$. Glycol[1:1] stripping $60 \mathrm{~min}$

(8) Lots of Cold E. Glycol clean room swabs - center to perimeter

(9) Back in $110^{\circ} \mathrm{C} \mathrm{NMP}+\mathrm{P}$. Glycol stripping $60 \mathrm{~min}$

(10) 4 x DI rinse

(11) MARCH 100W, $30 \mathrm{~min}$

(12) Inspect:

\section{Sample C:}

(13) $110^{\circ} \mathrm{C} \mathrm{NMP} \mathrm{+} \mathrm{P.} \mathrm{Glycol[1:1]} \mathrm{stripping} 60 \mathrm{~min}$

(14) Lots of Cold E. Glycol clean room swabs - center to perimeter

(15) Back in $110^{\circ} \mathrm{C} \mathrm{NMP}+\mathrm{P}$. Glycol stripping $60 \mathrm{~min}$

(16) $4 \times$ DI rinse

(17) MARCH 100W, $30 \mathrm{~min}$

(18) Inspect:

\section{Sample D:}

(19) $110^{\circ} \mathrm{C} \mathrm{NMP} \mathrm{+} \mathrm{P.} \mathrm{Glycol[1:1]} \mathrm{stripping} 60 \mathrm{~min}$

(20) Lots of Cold E. Glycol clean room swabs - center to perimeter

(21) Back in $110^{\circ} \mathrm{C} \mathrm{NMP}+\mathrm{P}$. Glycol stripping $60 \mathrm{~min}$

(22) 4 x DI rinse

(23) MARCH 100W, $30 \mathrm{~min}$

(24) Inspect: 


\section{SIS TESTMASK 04-29-2013 POGO \& CD INFORMATION}

"Layer1-Trilayer" "Layer2-Junction" "Layer3-Wire/Au"

\section{CD}

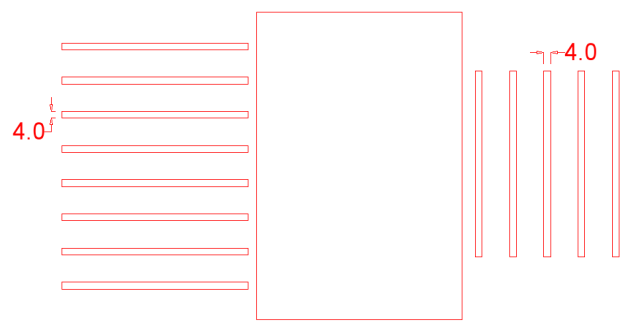

CD Layer=1 4.0um CAD

Mask is $4.2 \mathrm{um}$

Below is shown Layer $=2$ CD features. You will see an array of circles where from right to left, the circle diameter sizes are for each column: 4, 2, 1.4, $1.3,1.2,1.1,1.0,0.9,0.8,0.7,0.6$ um in CAD. The CD for this layer is the 1.0 um circles in CAD (which is the $5^{\text {th }}$ column from the left). These $1.0 u m$ circles in CAD are to become 1.0um diameter circles on the mask for Item-1; and 1.3um on the mask for Item-2.

CD Layer=2 circles $C D$ is 1.0 circle in $C A D$

(5th column from the left )

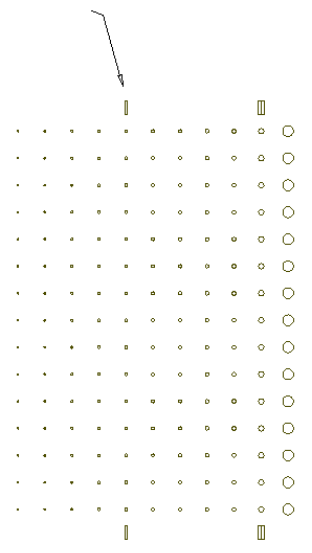

ALSO measure all the circles in one row. Note, in CAD from right to left they are: $4,2,1.4,1.3,1.2,1.1 .1 .0,0.9,0.8,0.7$, 0.6 I understand that only the 1.0 is a CD feature, but knowing how these other features come out in size on he mask would be very helpful 
Layer $=4$ CD features, with a spec'd 8um size in our CAD/GDS file, to become 8.3 um on the mask.

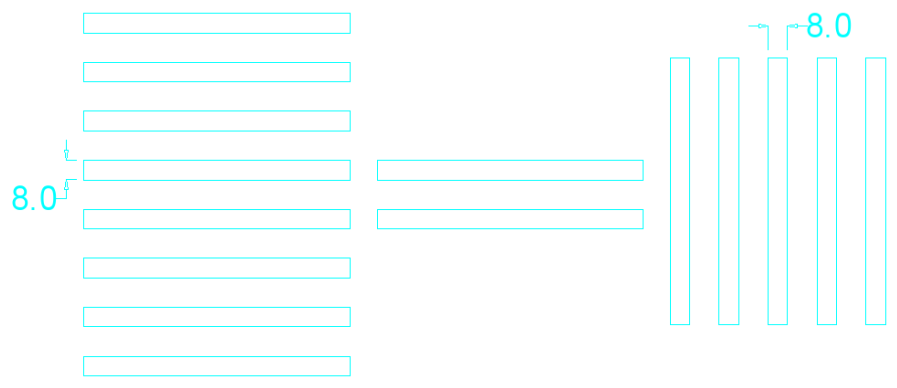

\section{Layer=4 8.0um CAD}

\section{POGO}

Pogo chips have a ground pad at one end. At the other end of the chips is pogo pad\#1... and all the way back adjacent to the ground pad is pogo\#6. There are also different pogo chip flavors. For this mask set, we have five different flavors:

\section{POGO1 (CHIP TYPE)}

Pogo\#1 $\quad \mathrm{N}=1$ Diameter $1.7 \mathrm{um}$ Wire=12um wide; $45 \mathrm{um}$ long

Pogo\#2

Pogo\#3

Pogo\#4

Pogo\#5

Pogo\#6

POGO2

Pogo\# 1

Pogo\#2

Pogo\#3

Pogo\#4

Pogo\#5

Pogo\#6

$\mathrm{N}=3$ Diameter 0.6um

$\mathrm{N}=3$ Diameter 0.6um

$\mathrm{N}=1$ Diameter $0.6 \mathrm{um}$

$\mathrm{N}=1$ Diameter 0.8um

$\mathrm{N}=0$ NO Junction

\section{(CHIP TYPE)}

$\mathrm{N}=1$ Diameter $1.7 \mathrm{um}$ Wire $=12 \mathrm{um}$ wide; $45 \mathrm{um}$ long

$\mathrm{N}=3$ Diameter $0.8 \mathrm{um}$

$\mathrm{N}=3$ Diameter $0.8 \mathrm{um}$

$\mathrm{N}=1$ Diameter 0.8um

$\mathrm{N}=1$ Diameter 1.0um

$\mathrm{N}=0$ NO Junction

Wire $=12 \mathrm{um}$ wide; $21 \mathrm{um}$ long ( 3

Wire $=12 \mathrm{um}$ wide; $45 \mathrm{um}$ long

Wire $=12 \mathrm{um}$ wide; $45 \mathrm{um}$ long

Wire $=12 \mathrm{um}$ wide; $45 \mathrm{um}$ long
Wire $=12 \mathrm{um}$ wide; $21 \mathrm{um}$ long $(3+)$

Wire $=12 \mathrm{um}$ wide; $21 \mathrm{um}$ long $(3+)$ repeat

Wire $=12 \mathrm{um}$ wide; $45 \mathrm{um}$ long

Wire $=12 \mathrm{um}$ wide; $45 \mathrm{um}$ long

Wire $=12 \mathrm{um}$ wide; $45 \mathrm{um}$ long 
POGO3

Pogo\# 1

Pogo\#2

Pogo\#3

Pogo\#4

Pogo\#5

Pogo\#6

POG04

Pogo\# 1

Pogo\#2

Pogo\# 3

Pogo\#4

Pogo\#5

Pogo\#6

POGO5

Pogo\# 1

Pogo\#2

Pogo\#3

Pogo\#4

Pogo\#5

Pogo\#6

\section{(CHIP TYPE)}

$\mathrm{N}=1$ Diameter $1.7 \mathrm{um}$ Wire $=12 \mathrm{um}$ wide; $45 \mathrm{um}$ long

$\mathrm{N}=3$ Diameter 1.0um

$\mathrm{N}=3$ Diameter 1.0um

$\mathrm{N}=1$ Diameter 1.0um

$\mathrm{N}=1$ Diameter $1.2 \mathrm{um}$

$\mathrm{N}=0$ NO Junction

Wire $=12$ um wide; 21 um $\times 3$ long (+7 long)

Wire $=12 \mathrm{um}$ wide; $21 \mathrm{um}$ long $(3$

Wire $=12 \mathrm{um}$ wide; $45 \mathrm{um}$ long

Wire $=12 \mathrm{um}$ wide; $45 \mathrm{um}$ long

Wire $=12 \times 33 u m+\underline{100 \times 100 u m ~ s q u a r e ~}$

\section{(CHIP TYPE)}

$\mathrm{N}=1$ Diameter 1.0um

$\mathrm{N}=3$ Diameter $1.7 \mathrm{um}$

$\mathrm{N}=3$ Diameter 1.7um

$\mathrm{N}=1$ Diameter 1.4um

$\mathrm{N}=1$ Diameter $1.7 \mathrm{um}$

$\mathrm{N}=0$ NO Junction
Wire $=12 \mathrm{um}$ wide; $45 \mathrm{um}$ long

Wire $=12 \mathrm{um}$ wide; $21 \mathrm{um} \times 3$ long (+7 long)

Wire $=12 \mathrm{um}$ wide; $21 \mathrm{um}$ long ( 3

Wire $=12 \mathrm{um}$ wide; $45 \mathrm{um}$ long

Wire $=12 \mathrm{um}$ wide; $45 \mathrm{um}$ long

Wire $=12 \times 33 u m+\underline{160 \times 125}$ um square

\section{(CHIP TYPE)}

$\mathrm{N}=1$ Diameter $0.6 \mathrm{um}$ Wire $=12 \mathrm{um}$ wide; $45 \mathrm{um}$ long

$\mathrm{N}=3$ Diameter $0.8 \mathrm{um}$

$\mathrm{N}=1$ Diameter 1.1um

$\mathrm{N}=1$ Diameter 1.6um

$\mathrm{N}=1$ Diameter 2.3um

$\mathrm{N}=1$ Diameter $3.2 \mathrm{um}$
Wire $=12 \mathrm{um}$ wide; $45 \mathrm{um}$ long

Wire $=12 \mathrm{um}$ wide; $45 \mathrm{um}$ long

Wire $=12 \mathrm{um}$ wide; $45 \mathrm{um}$ long

Wire $=12 \mathrm{um}$ wide; $45 \mathrm{um}$ long

Wire $=12 \mathrm{um}$ wide; $45 \mathrm{um}$ long 


\section{Appendix V}

\section{Source Code and Documentation}

This appendix contains all the source code and documentation for both: (a) the IV plotting software (which determines $R_{N} A$ and $R_{S G} / R_{N}$ ) and, (b) the code used to determine relative dissociation of a nitrogen plasma. Both programs were developed using MATLAB® R2012a for 64-bit Linux, and are likely to work on other Linux MATLAB® installations. The programs may work under OS X, but will not work under any Microsoft Windows installations without modifications due to the use of system calls.

\section{Plotting Software}

The software plots the IVs, recorded by the GRAPHTEC GL900 MIDI logger, that have been stored as comma separated variable (CSV) files. Figure V1 shows the graphical user interface (GUI) of the program. In addition to plotting the IV characteristics, the program can also calculate $R_{N} A$ (for a user input of diameter) and $R_{S G} / R_{N}$. The function of the GUI buttons from top to bottom are listed below.

Browse for CSV button: Opens a dialog to select CSV files for plotting.

Plot Color: Opens a drop down list to select the color used for the current plot.

\# Junctions: This opens a drop down menu where the use can select the number 
of junctions in series for the particular test element. This tells the program how to calculate $R_{N}$ and $R_{S G}$.

Divide by 100: For high current density junctions, our bias box has the option to divide $V_{i}$ by 100 . When checked, $I_{j}$ is divided by 100 .

Plot in New Window: Plots IV in a separate window instead of the included axes.

Clear Axes: Clears current axes before plotting next IV.

Remove Jc: Removes the Josephson current between zero volts and gap voltagethis was found useful for comparing multiple junctions.

Show Rsg/Rn: Displays the calculated value of $R_{S G} / R_{N}$ on the plot.

Show RnA: Displays the calculated value of $R_{N} A$ on the plot.

Save Plot: When checked, every plot is automatically saved as an encapsulated postscript (EPS) file in the folder which the program resides. The file name is the same as the CSV file.

Plot: Generates the IV plot.

Junction Diameter $(\mu \mathbf{m})$ : User enters the junction diameter for $R_{N} A$ calculations.

Recalc: Recalculates the junction resistance, $R_{N} A$, and $R_{S G} / R_{N}$ based on the user input for junction diameter.

\section{Plotting Code}

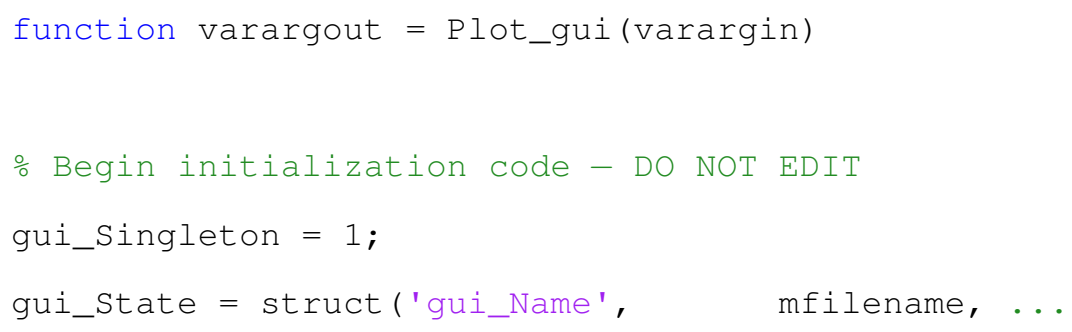




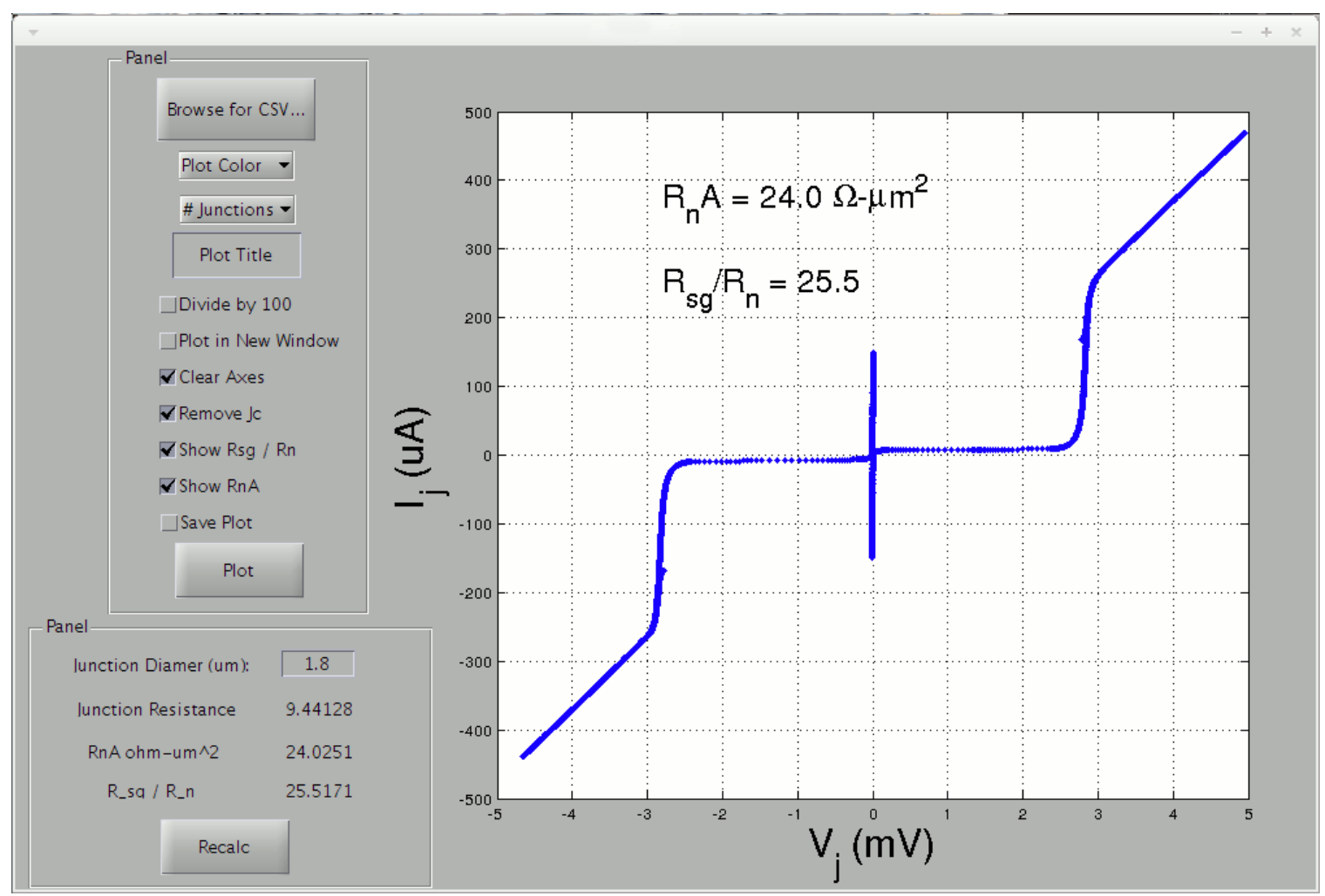

Figure V1: GUI of IV plotting software.

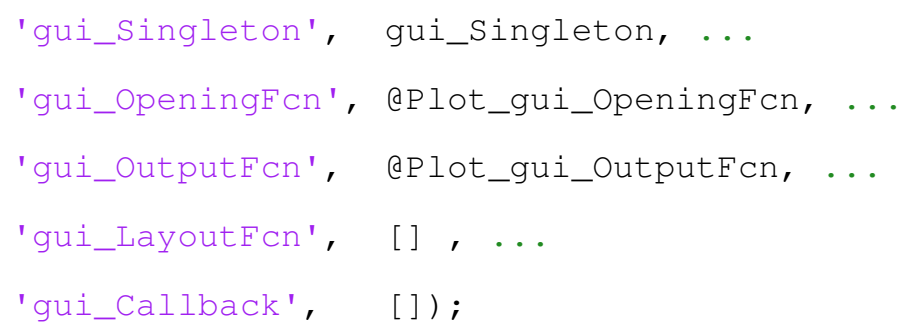

if nargin \&\& ischar (varargin $\{1\}$ )

gui_state.gui_Callback = str2func(varargin $\{1\})$;

end

if nargout

[varargout $\{1:$ nargout $\}]=$ gui_mainfon(gui_state, varargin $\{:\})$;

else

gui_mainfon (gui_state, varargin $\{:\})$;

end

\% End initialization code - DO NOT EDIT 


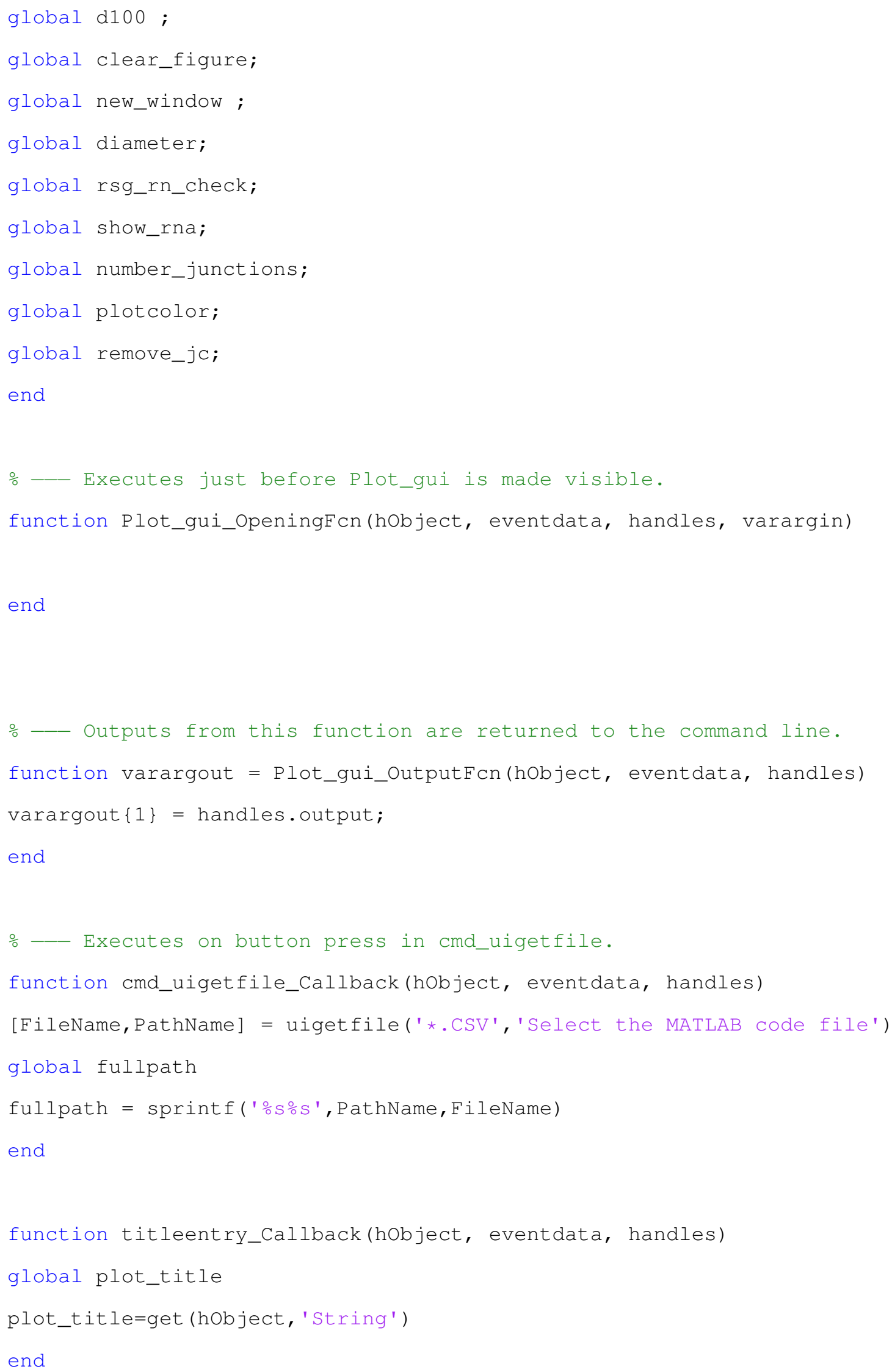




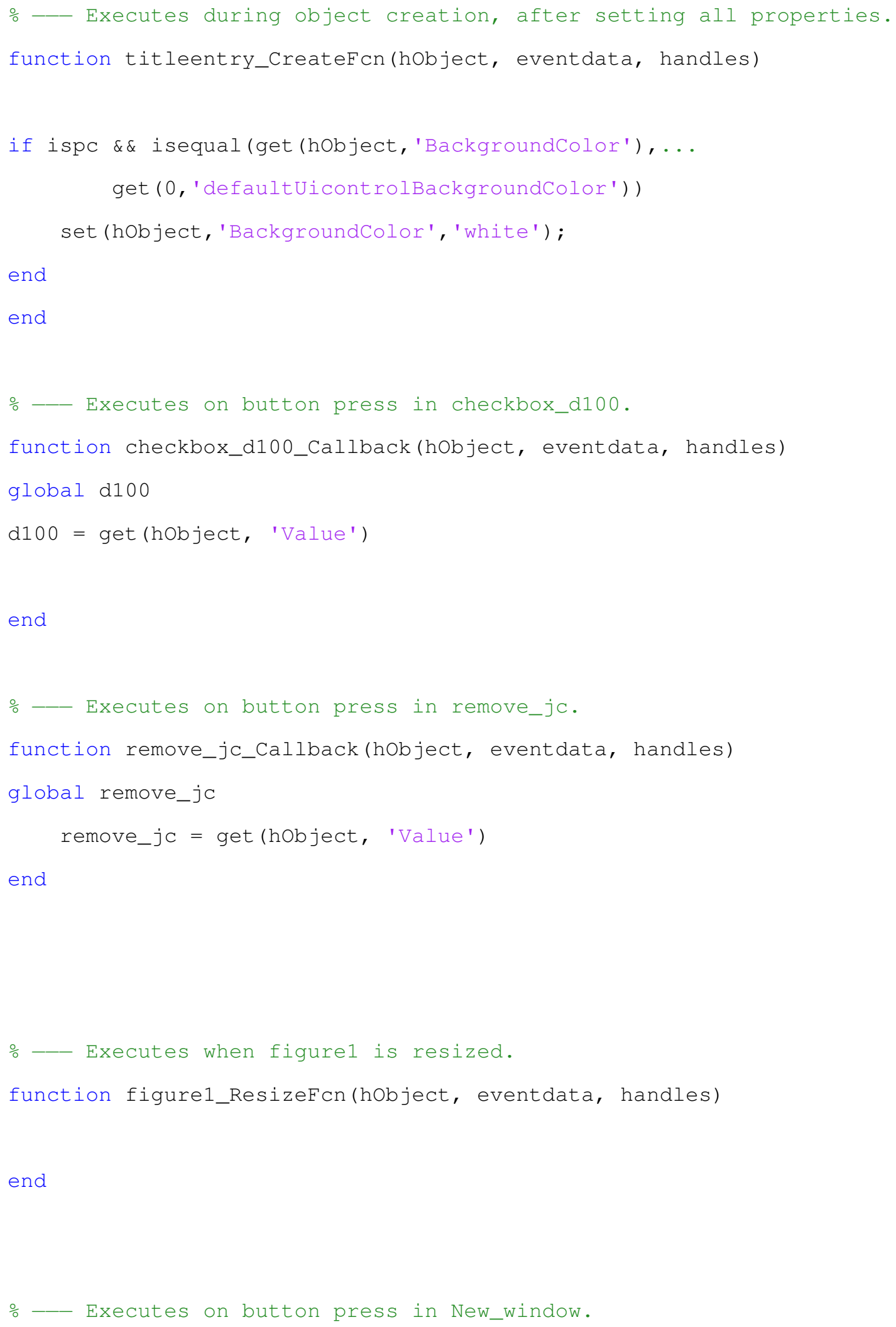




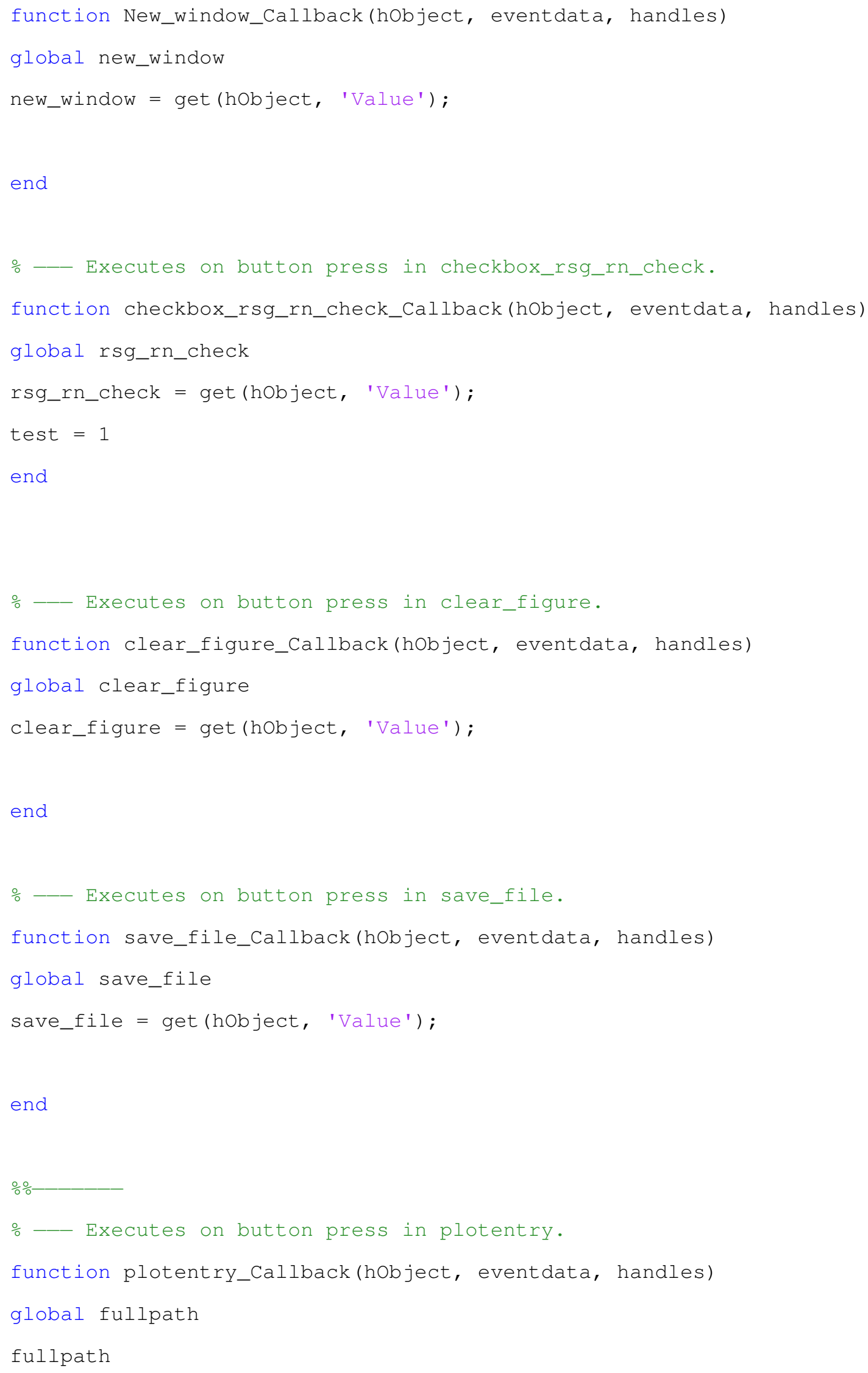




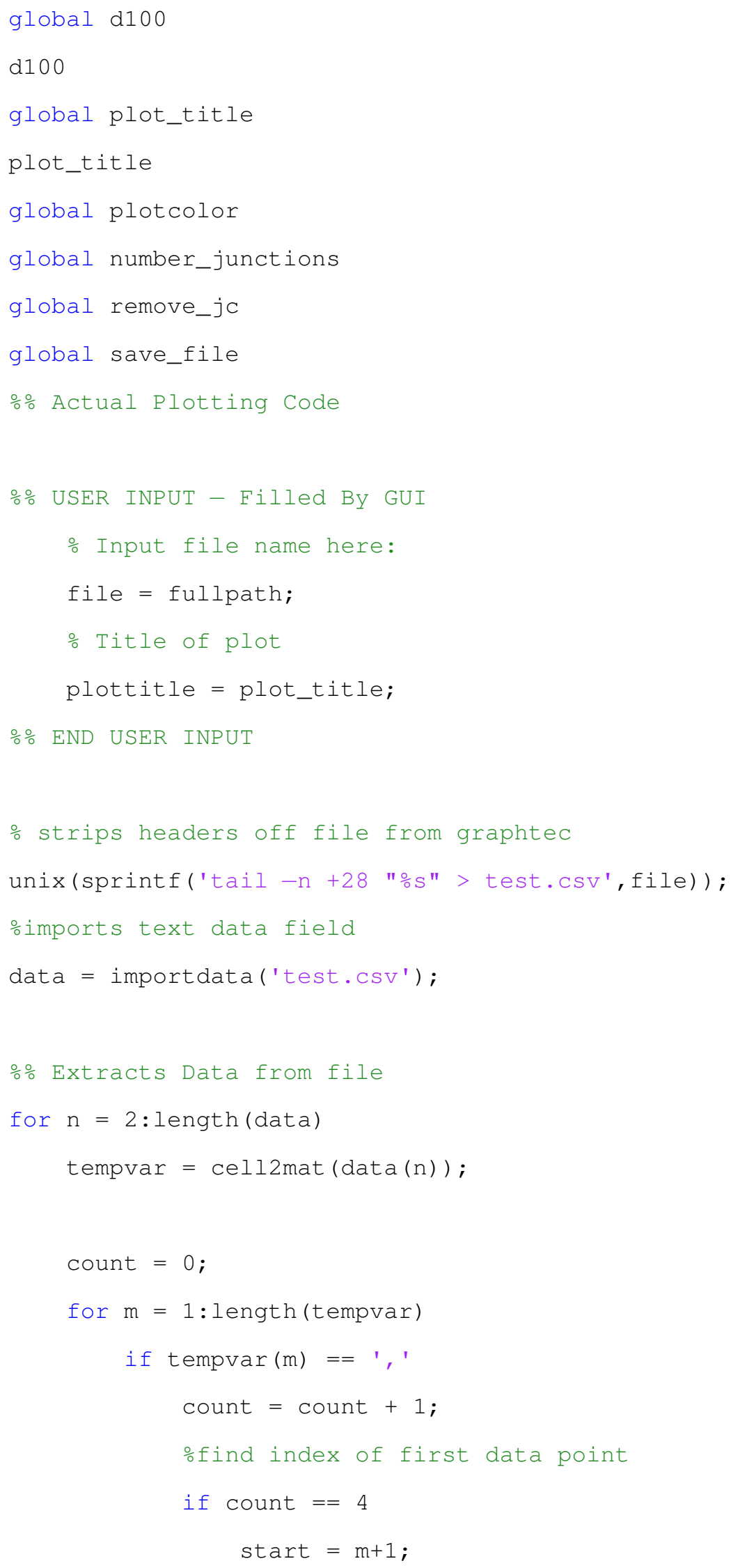




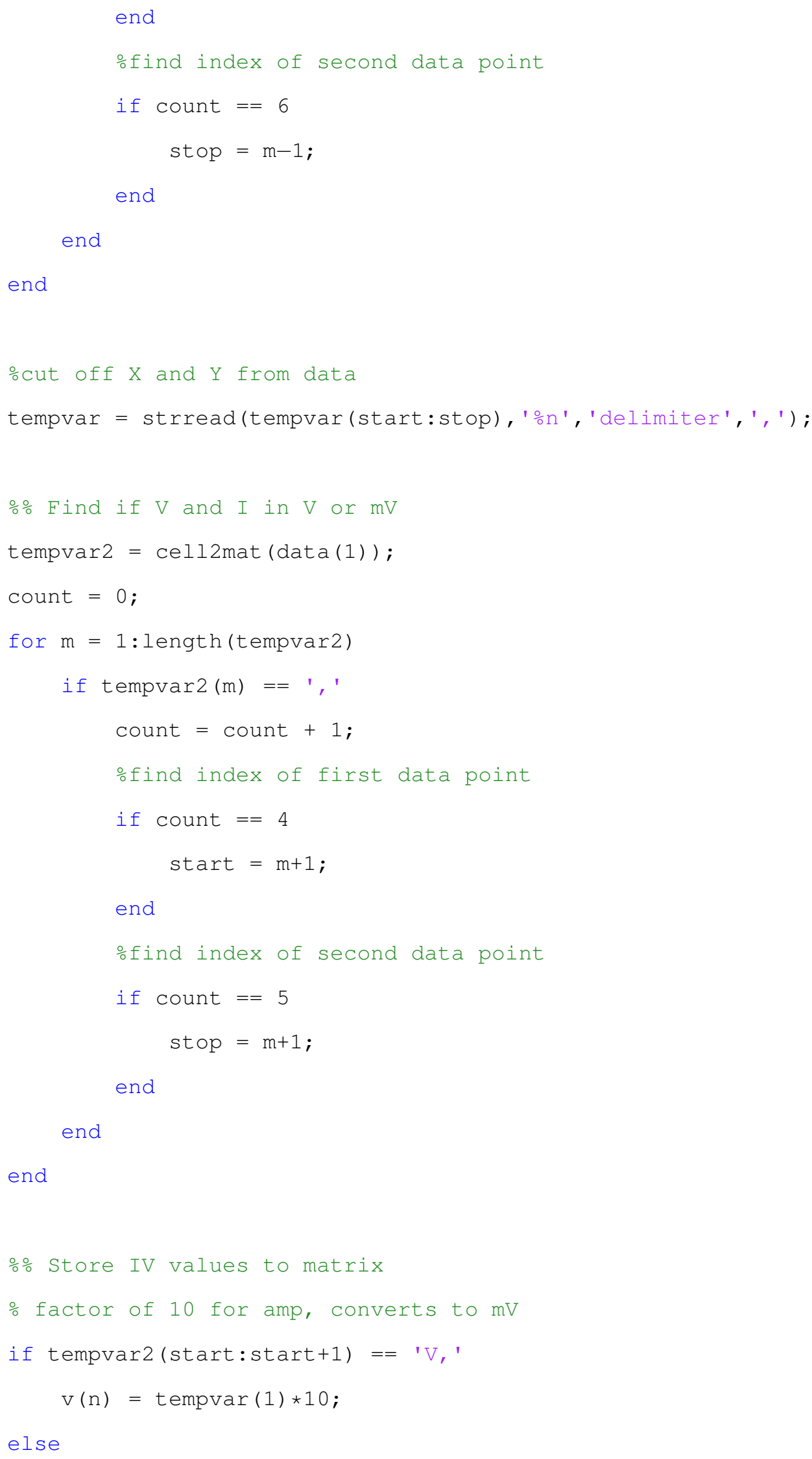




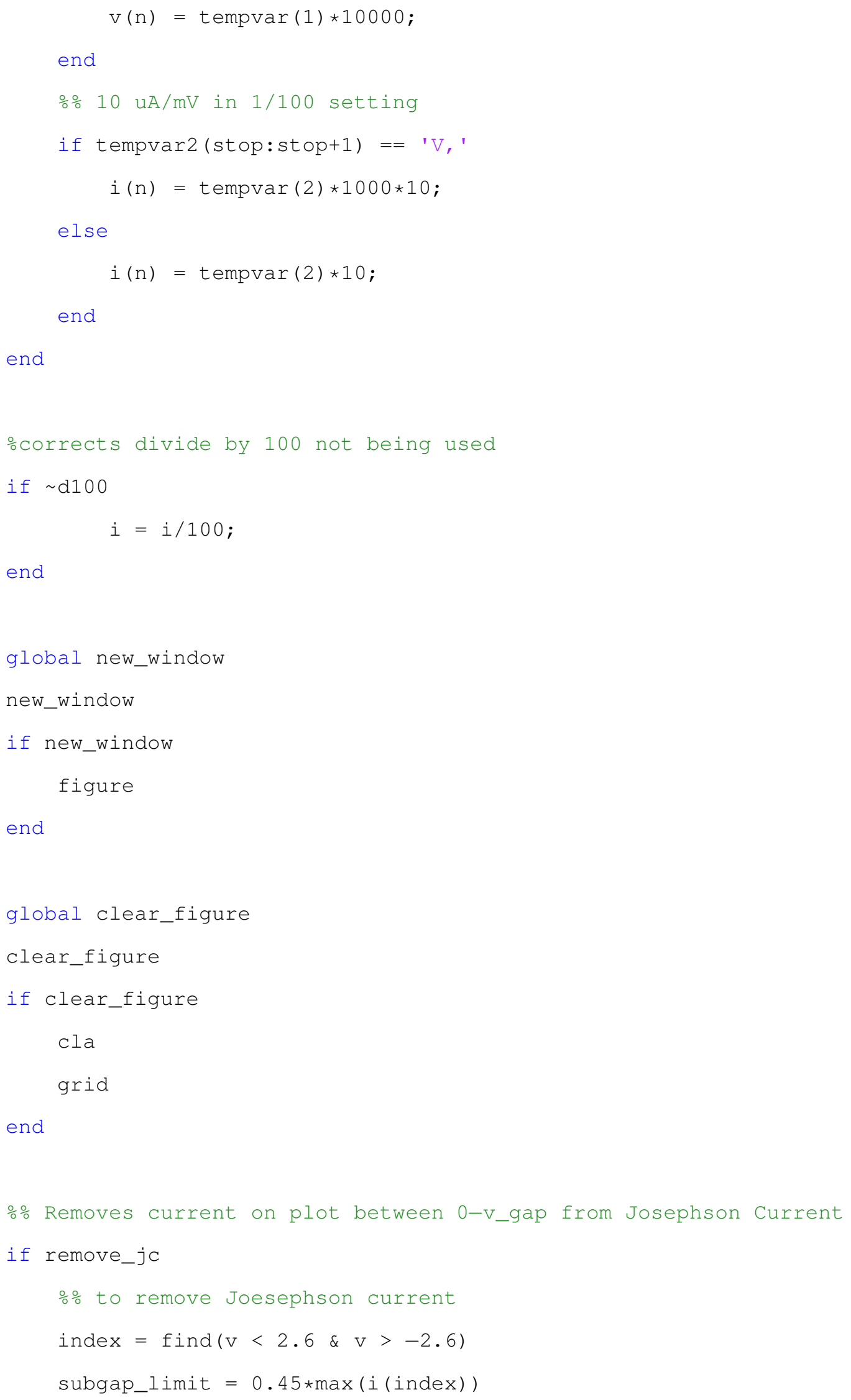




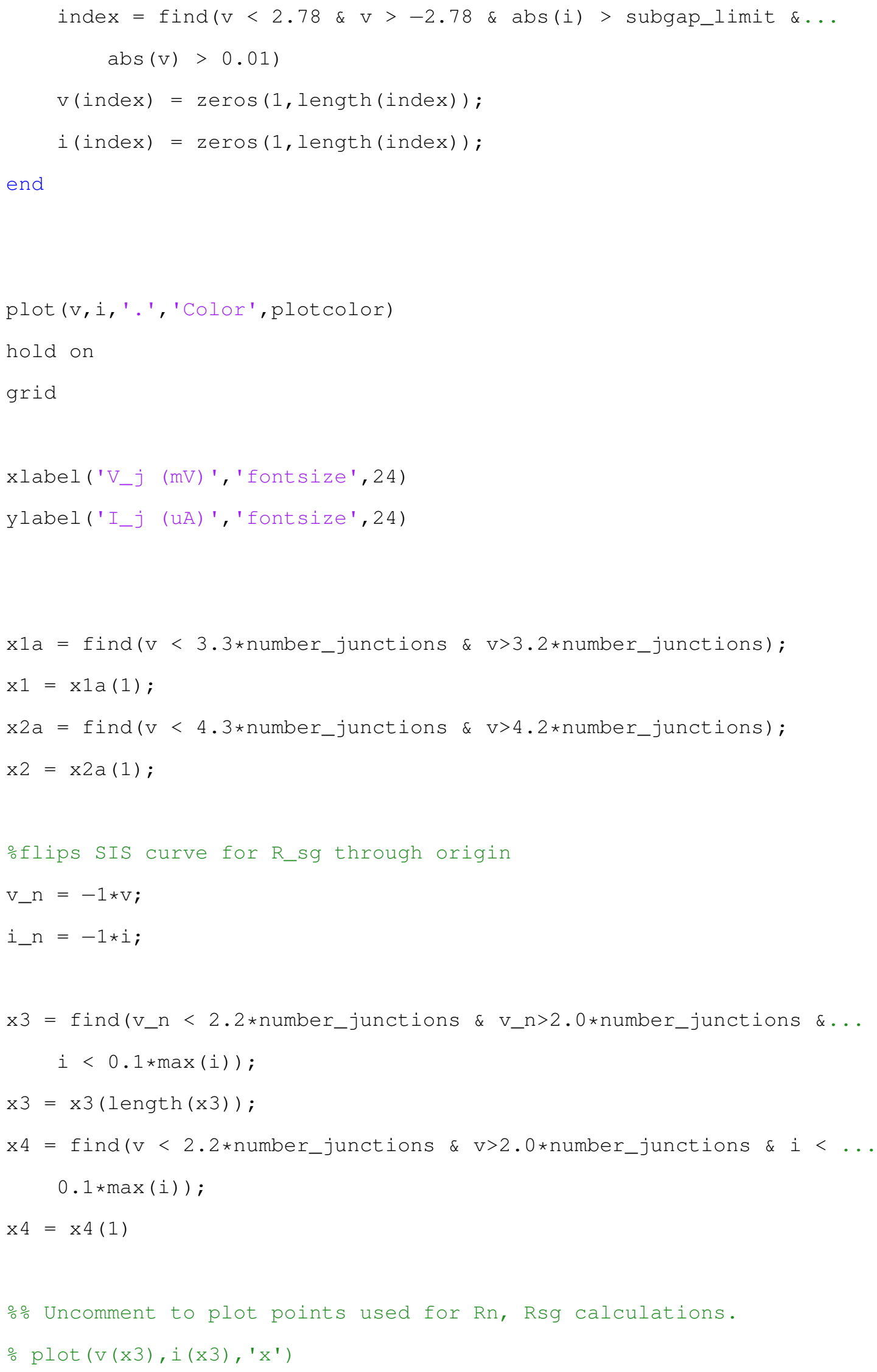




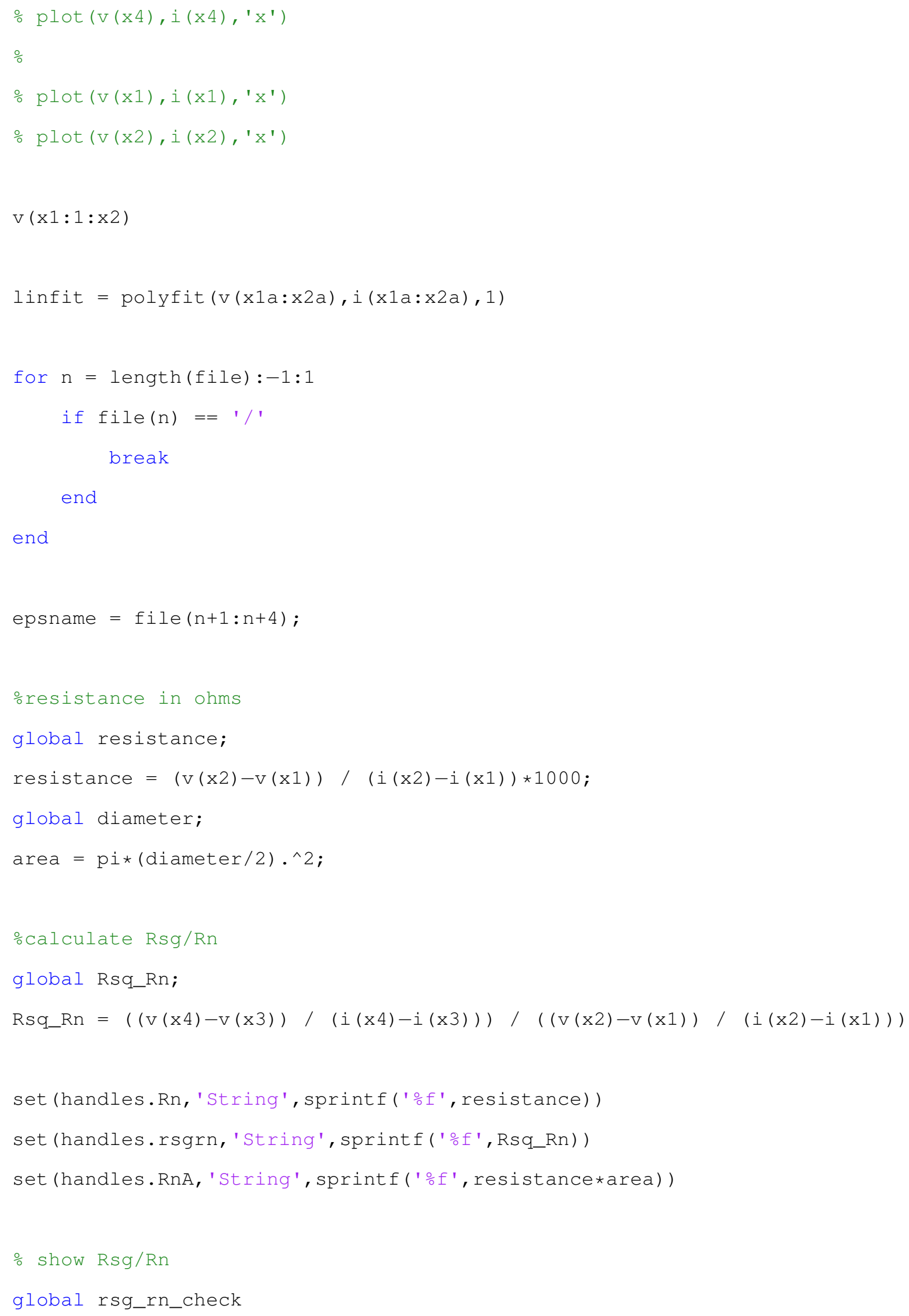




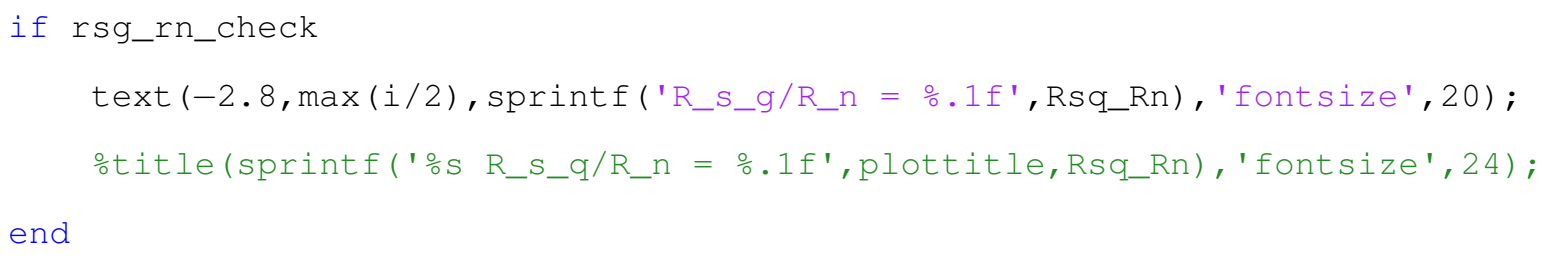

end

function Diameter_Callback(hobject, eventdata, handles)

global diameter;

diameter=str2double (get (hobject, 'String'))

\% hobject handle to Diameter (see GCBO)

\% eventdata reserved - to be defined in a future version of MATLAB

\% handles structure with handles and user data (see GUIDATA) 


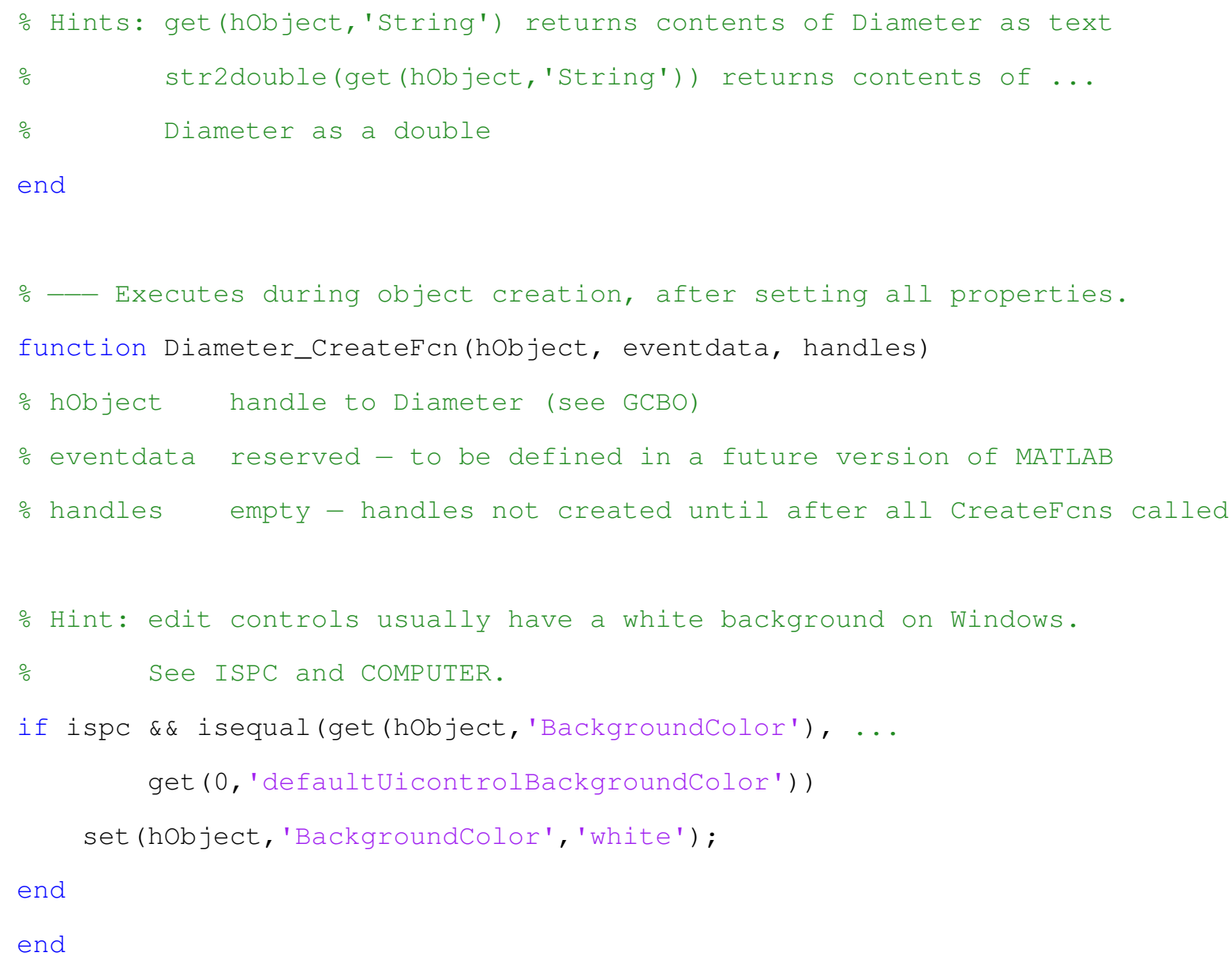

ㄴ- Executes on button press in checkbox_show_rna.

function checkbox_show_rna_Callback(hobject, eventdata, handles) 


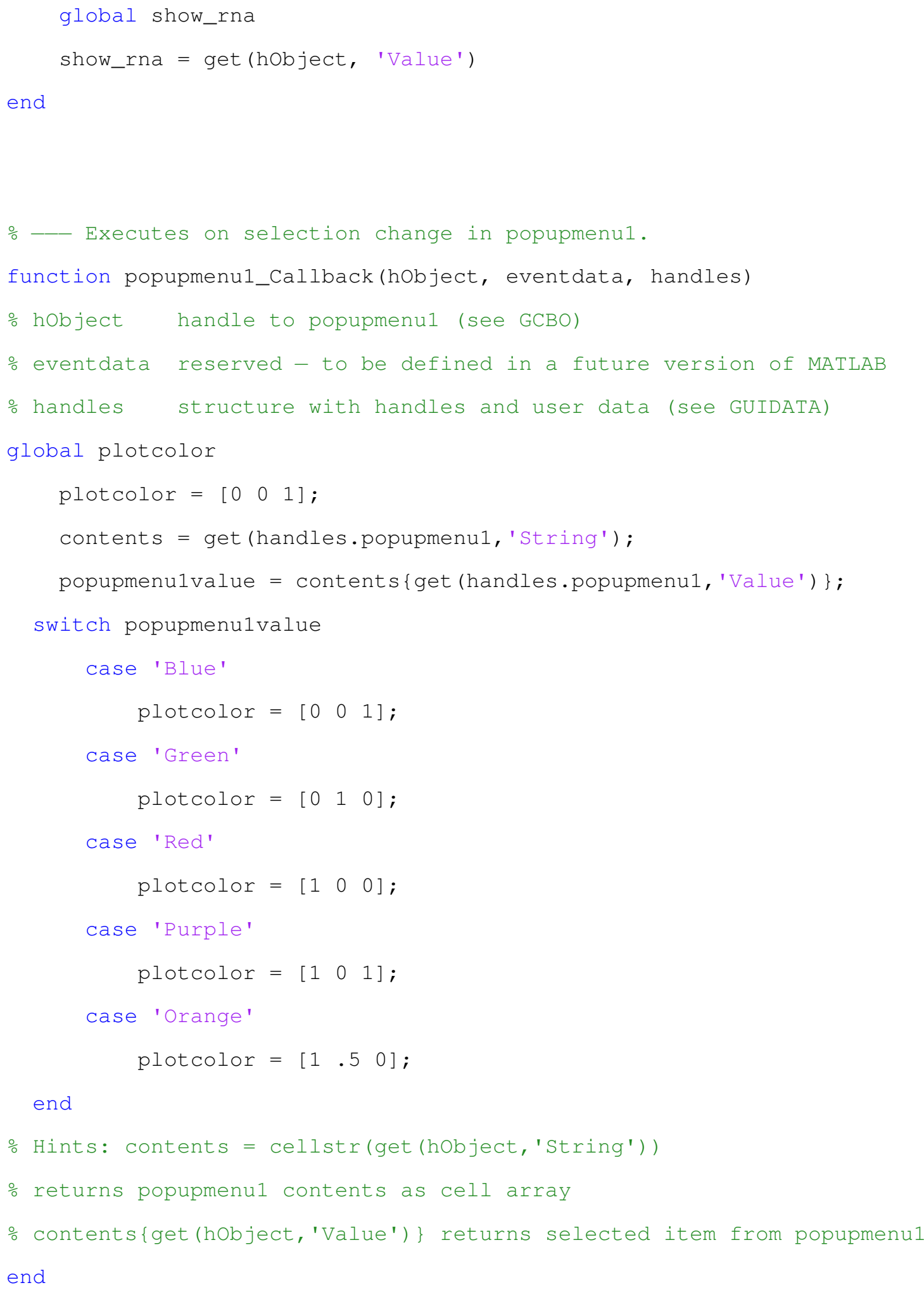




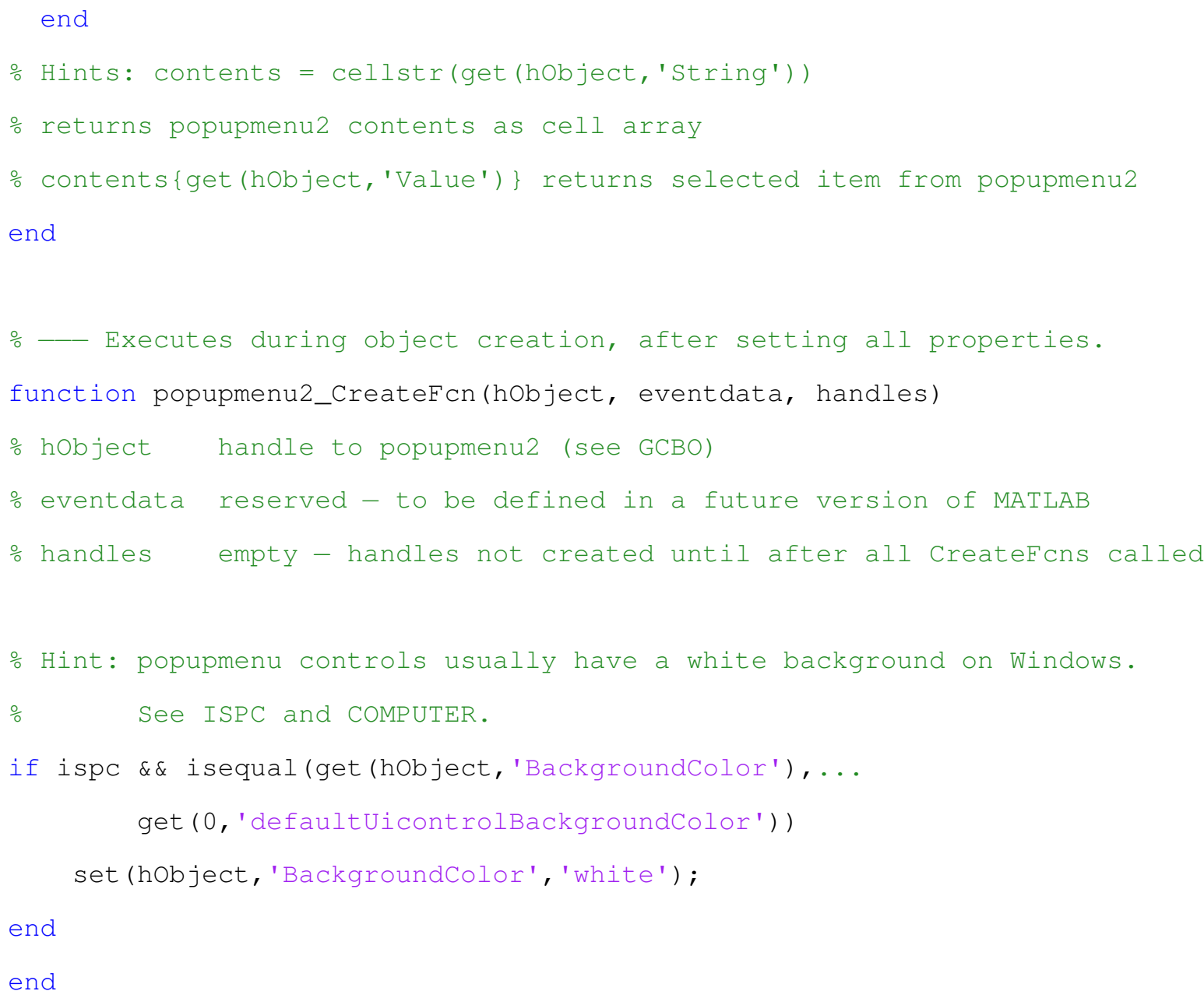




\section{Code for Relative Dissociation Plots}

The following script reads all the tab-delimited files in its residing folder, recorded by the Ocean Optics USB4000 spectrometer using the OceanView spectroscopy operating software package, and calculates the relative dissociation. The results are stored in the array 'data_p', the columns from left to right store the following information: (1) operating pressure in $\mathrm{mT}$, (2) RF power in $\mathrm{W},(3) \mathrm{DC}$ current in $\mathrm{A}$, and (4) relative dissociation. The script will calculate this for every data set in the folder so long as the file names begin with the following format; (pressure)_(power)_(current), where periods are represented by 'p'. Ex: 5_200_2p5_Plasma.txt for a $5 \mathrm{mT}, 200 \mathrm{~W}$, 2.5A plasma. A notable benefit of this algorithm is that it can be applied to analyze arbitrarily large data sets.

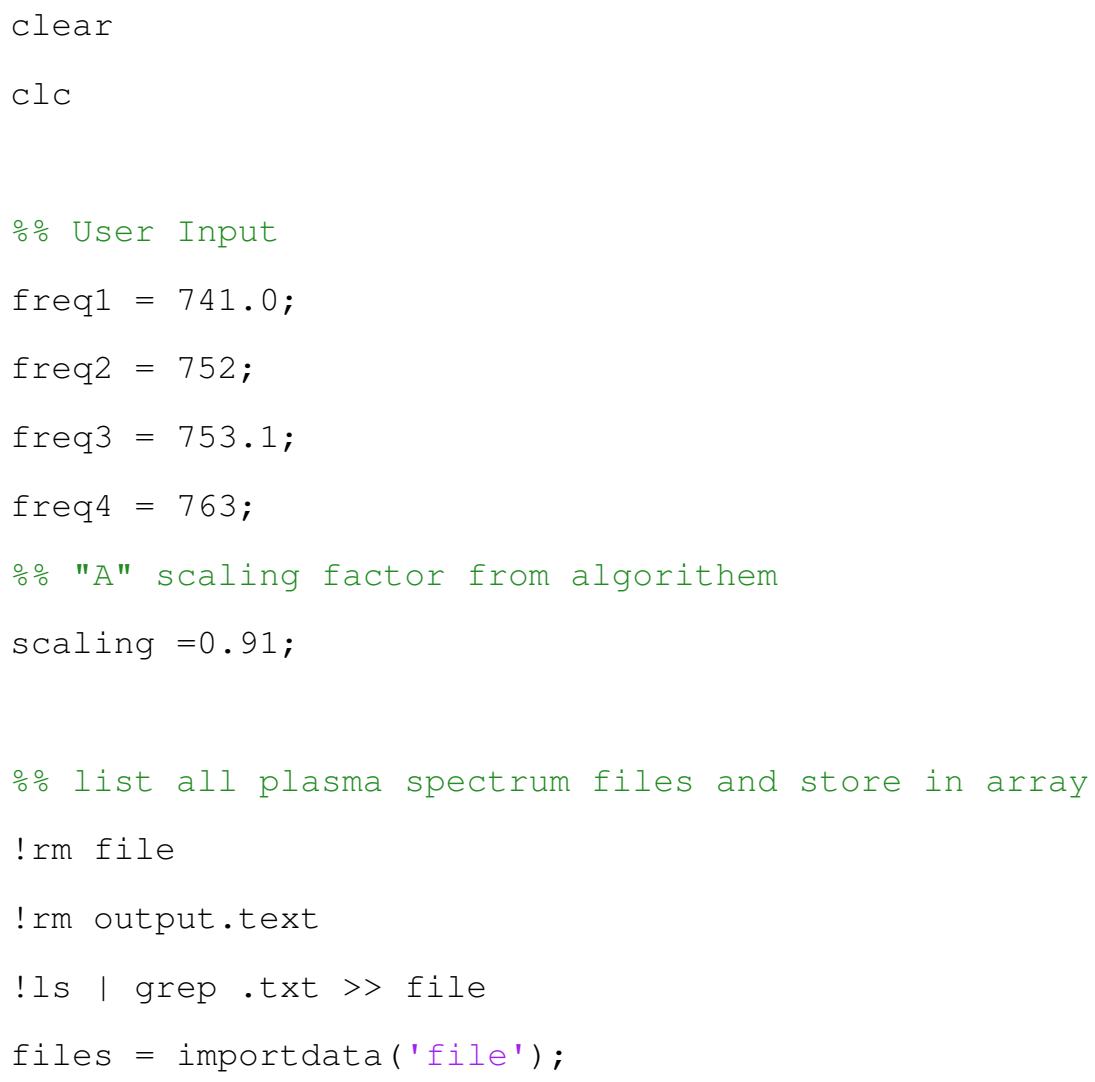




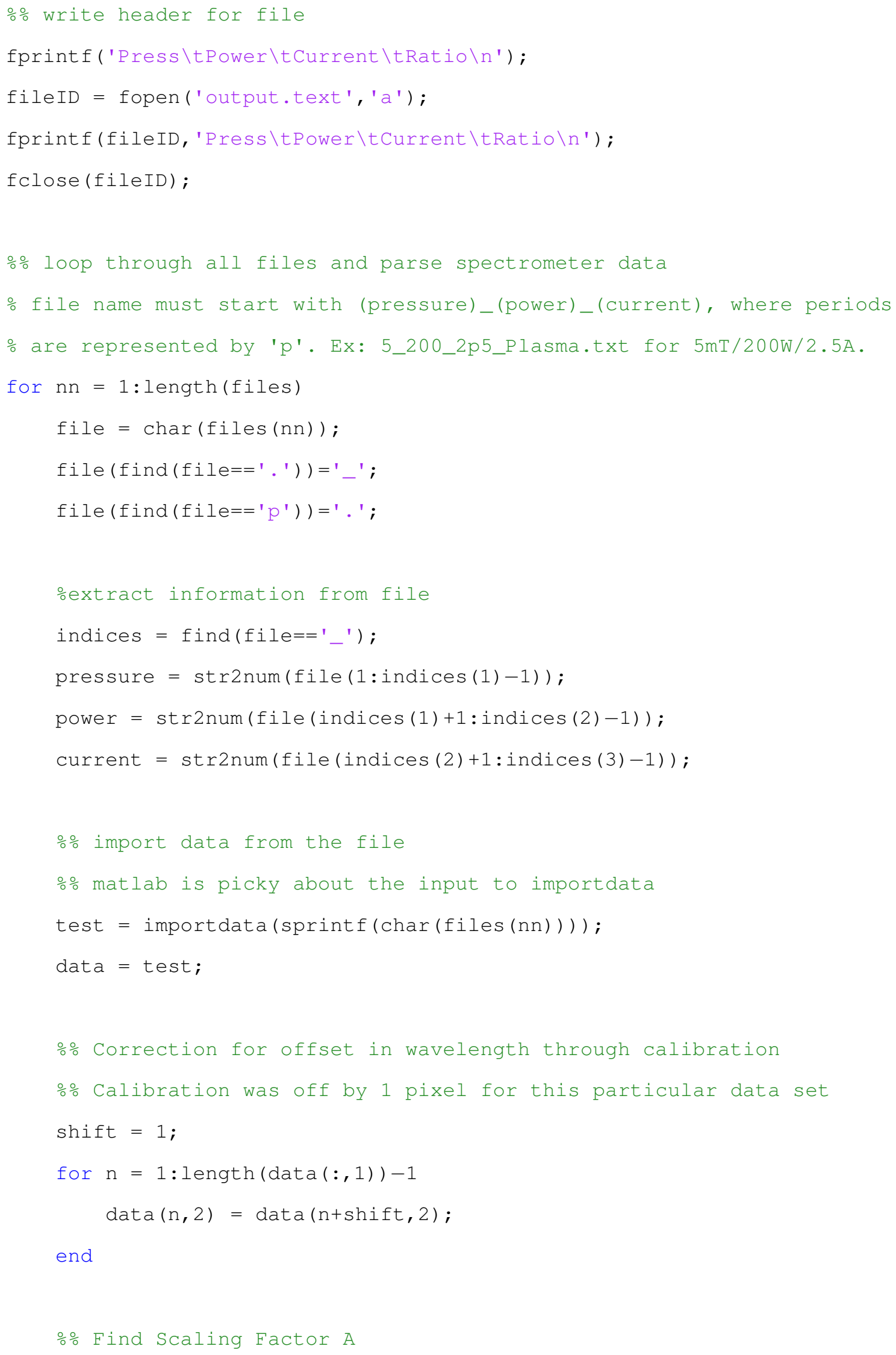




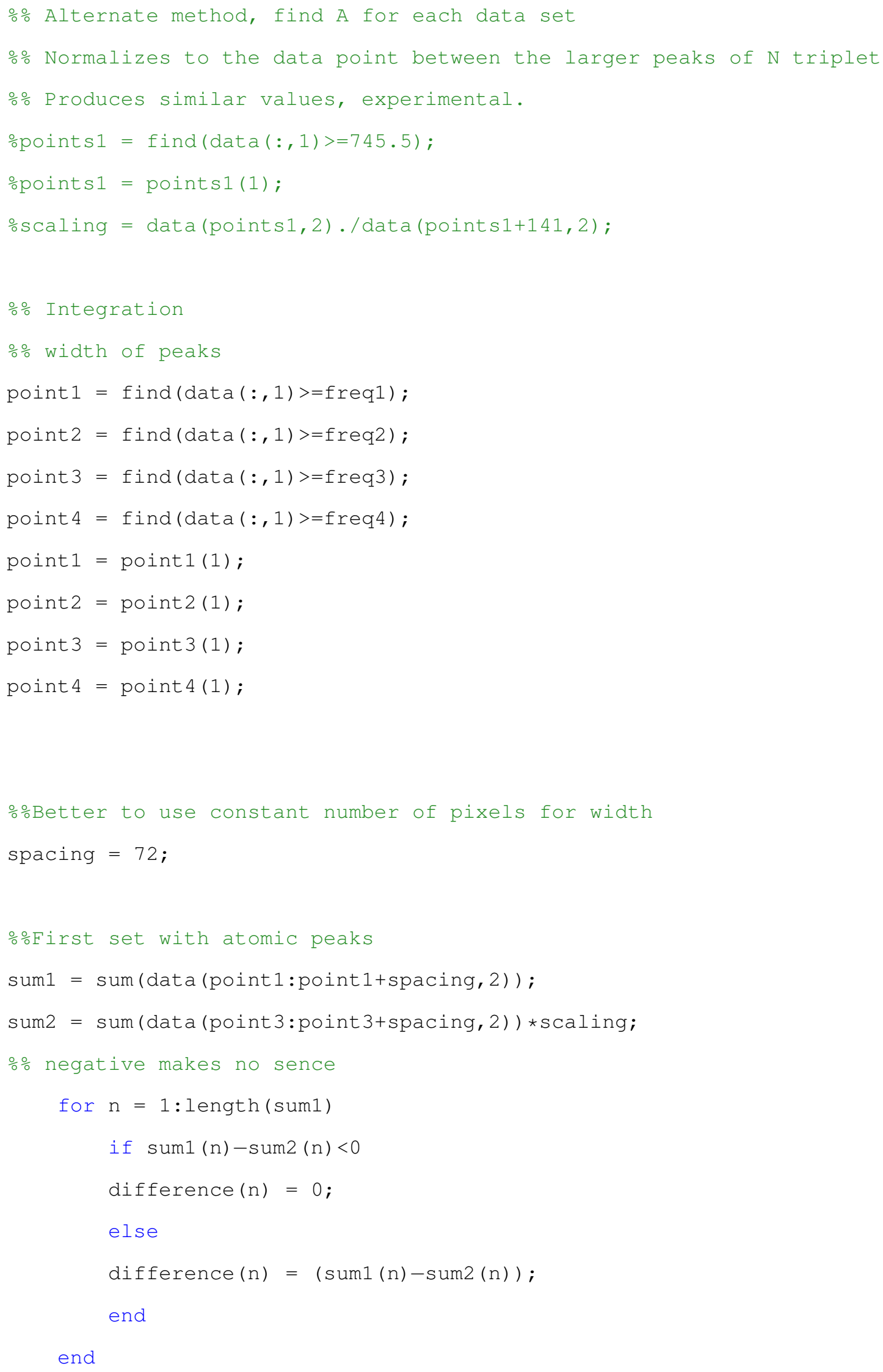




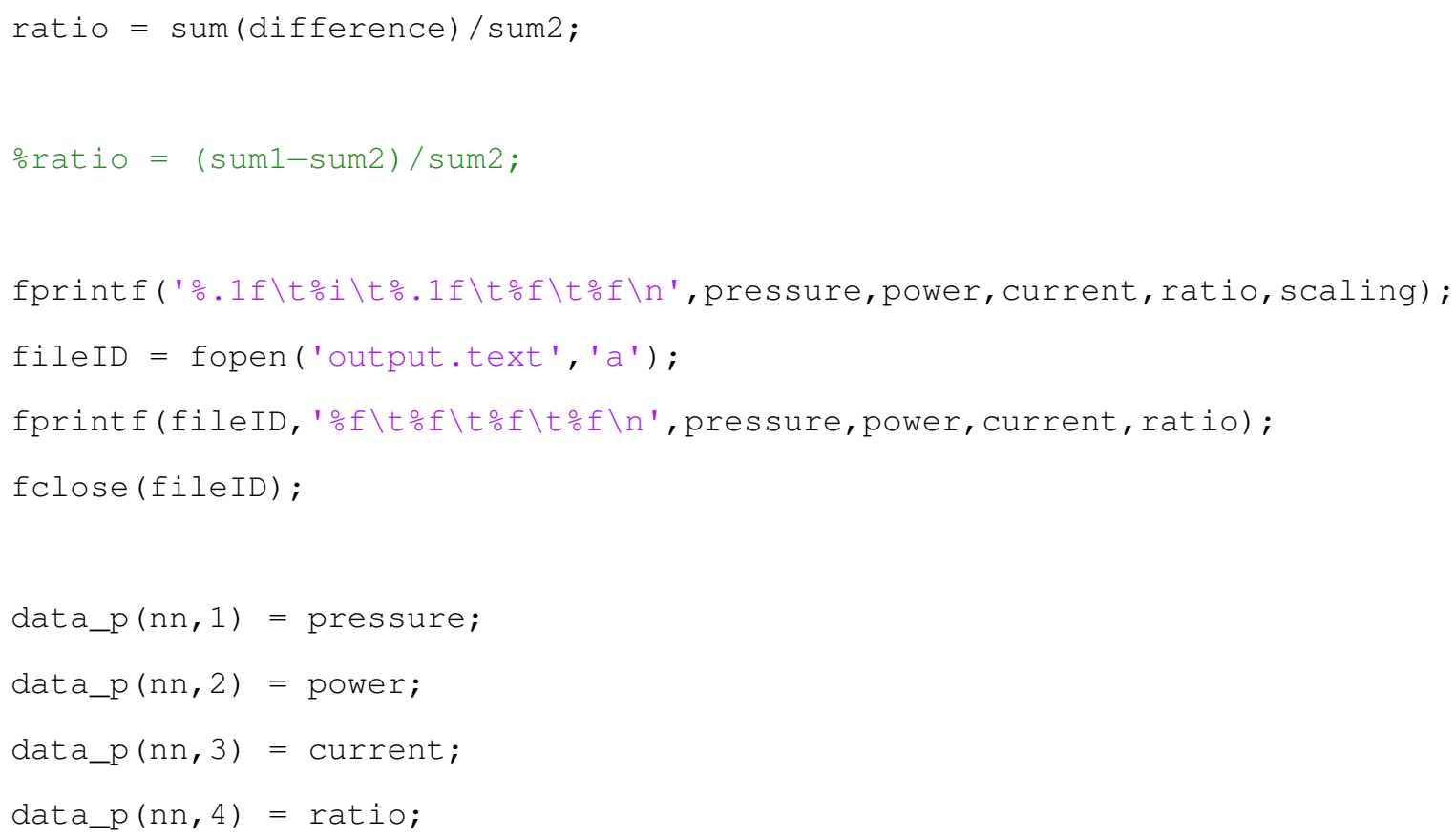

end 